\title{
WestVirginiaUniversity
}

THE RESEARCH REPOSITORY @ WVU

Graduate Theses, Dissertations, and Problem Reports

2011

\section{Multi Agent System Based Fault Location in a Smart MicroGrid System}

\author{
Seetaram Alwala \\ West Virginia University
}

Follow this and additional works at: https://researchrepository.wvu.edu/etd

\section{Recommended Citation}

Alwala, Seetaram, "Multi Agent System Based Fault Location in a Smart MicroGrid System" (2011). Graduate Theses, Dissertations, and Problem Reports. 3346.

https://researchrepository.wvu.edu/etd/3346

This Thesis is protected by copyright and/or related rights. It has been brought to you by the The Research Repository @ WVU with permission from the rights-holder(s). You are free to use this Thesis in any way that is permitted by the copyright and related rights legislation that applies to your use. For other uses you must obtain permission from the rights-holder(s) directly, unless additional rights are indicated by a Creative Commons license in the record and/ or on the work itself. This Thesis has been accepted for inclusion in WVU Graduate Theses, Dissertations, and Problem Reports collection by an authorized administrator of The Research Repository @ WVU. For more information, please contact researchrepository@mail.wvu.edu. 


\title{
Multi Agent System Based Fault Location in a Smart MicroGrid System
}

\author{
Seetaram Alwala \\ Thesis submitted to the \\ College of Engineering and Mineral Resources \\ Dt West Virginia University \\ In partial fulfillment of the requirements \\ Ior the degree of
}

\section{Master of Science}

in

Electrical Engineering

Dr. Ali Feliachi, Chair

Dr. Muhammad A. Choudhry

Dr. Jignesh M. Solanki

Lane Department of Computer Science and Electrical Engineering

\section{Morgantown, West Virginia \\ 2011}

Keywords: Fault Location, MicroGrid, Multi Agent technology, OpenDSS, JADE

Copyright 2011 Seetaram Alwala 


\title{
ABSTRACT \\ Multi Agent System Based Fault Location in a Smart MicroGrid System
}

\author{
Seetaram Alwala
}

The electric power grid is inherently a huge network that has been going through a lot of transformation because of its restructuring and increase in the deployment of Distributed Generation. The steep increase in electricity demand and the large financial losses caused by the blackouts has initiated research towards increasing the security and robustness of the electric grid and making it a Smart Grid. Smart Grids might incorporate MicroGrids, which can be defined as low to medium voltage networks that contain aggregation of certain loads and Distribution Generation units that can operate in either grid-connected or islanded mode. One of the leading technologies to deploy intelligence in the electric grid is the agent technology. Multi Agent systems are basically a congregation of individual agents that can sense changes in their environment and autonomously make decisions to meet their design objectives. The technological advancement in the agent technology can provide solutions for a wide range of Smart Grid activities.

In a MicroGrid, a single fault can lead to catastrophic, cascading events that could result in a blackout. This makes fault location an important problem to solve. The penetration of Distributed Generation units causes a two way power flow in the traditional radial distribution system. This, coupled with the inherent unbalanced nature of the distribution system, makes the fault location a difficult problem to solve.

In this thesis, a Multi Agent system has been formulated to determine the precise fault location and identify faulty zones. The algorithm for the fault detection is based on the magnitude of the sequence components of currents and current directions during a fault in the system.

The agent model for the fault detection has been implemented in the software named Java Agent Development Framework. The distribution test system used for this work is the IEEE 37 bus system. In order to simulate the MicroGrid, Distributed Generation units are added at various nodes in the test system. This circuit has been simulated in a distribution system simulation software, OpenDSS. All the possible fault cases for different penetration levels of Distributed Generation are considered and tested. Some of these fault cases have been presented in this thesis. 


\section{DEDICATION}

I would like to dedicate this thesis to my parents and sister,

For their unhindered support, encouragement and love

$\&$

To Mamaji, Sri Prabhakar Rao Garu,

Without whom my journey to USA would not have been possible.

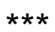

Grand pa, you are deeply missed! 


\section{ACKNOWLEDGEMENT}

I would like to express my sincere thanks and gratitude to my research advisor Dr. Ali Feliachi for making me a part of APERC and work under his esteemed guidance. My discussions with Dr. Feliachi have taught me a lot of new things in electrical power system and inspired me to do good research work. I also wish to express my sincere thanks to Dr. Muhammad A. Choudhry for his invaluable suggestions and the enormous amount of time he has given me to discuss power system concepts. Further, I sincerely thank Dr. Jignesh Solanki for being in my advisory committee and introducing the concepts of Multi Agent system to me. I would also like to specially thank, Dr. Noore and Brian Powell for giving me a GTA with CS101 and guiding my class lectures in the class.

I would also like to express my thanks to all APERC lab mates Sridhar, Manasa, Rabie, Shola, Naveen and Vaidhy for the healthy discussions and their suggestions towards my research. I cannot thank my roommates and best friends Krishna, Anand and Dandu enough for their great care, support and help.

I would specially like to acknowledge my friend Vamsi Krishna Kamaraju and Stephani Smith from WVU creative writing centre for proof reading my document.

I owe my heartfelt regards to my mom, dad, pedamma and sister for their unhindered love, support and encouragement. I cannot thank my Uncle Sri Prabhakar Rao Garu and brother in law Chaitanya enough for the great help they have given me and my family. I will be forever indebted to them. 


\section{Table of Contents}

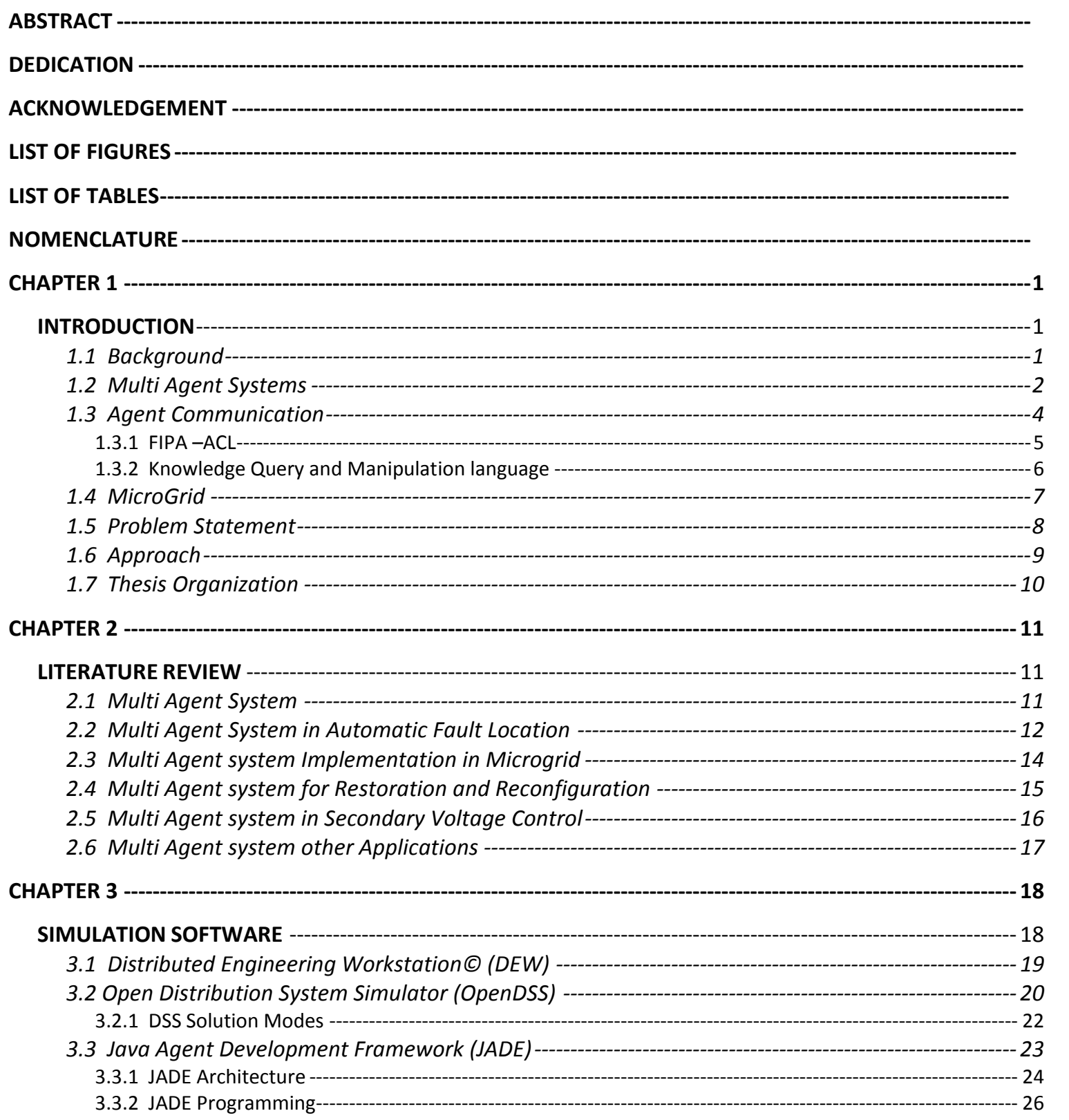

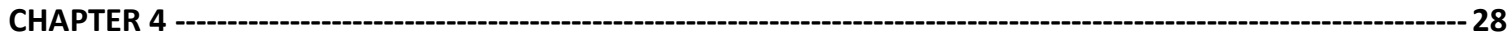

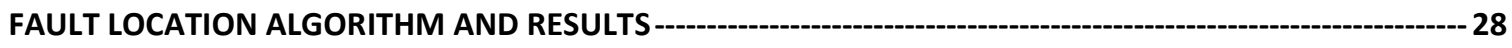

4.1 Multi Agent System Architecture ---------------------------------------------------------------------------------- 28

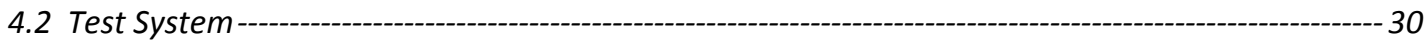

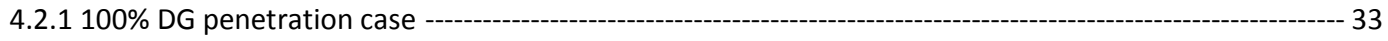

4.2.2 75\% DG Penetration Case--- 38

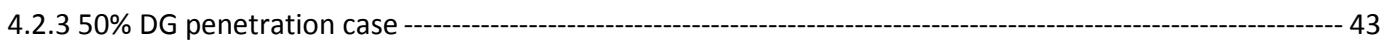

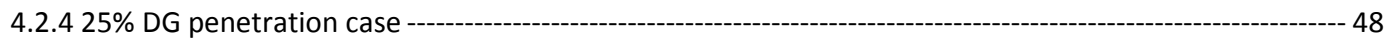

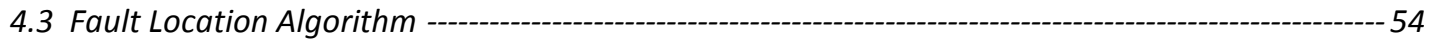

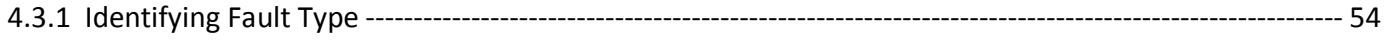

4.3.2 Determining The Fault Location - 55 


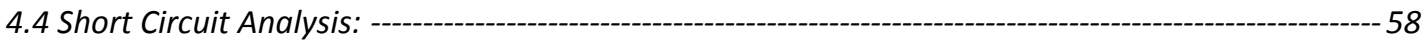

4.4.1 Short Circuit Analysis 50\% DG Penetration -----_- 58

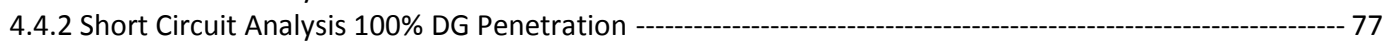

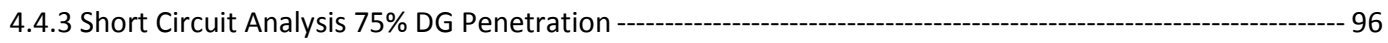

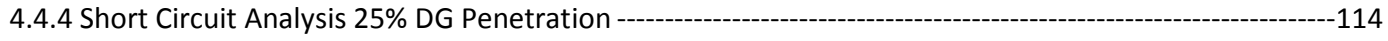

4.5 Fault Location Test Cases ------------------------------------------------------------------------------------1 133

4.5.1 Single line to ground Fault at node 713---133

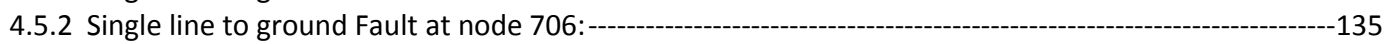

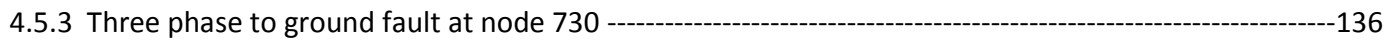

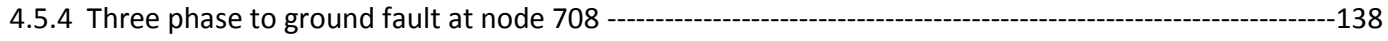

4.5.5 Line to Line fault at node 738 --140

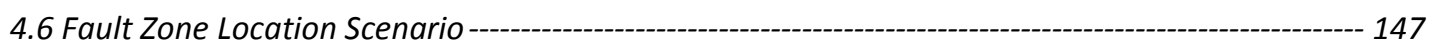

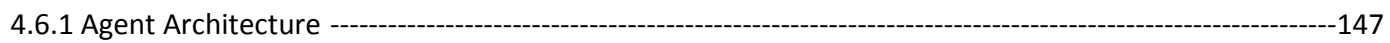

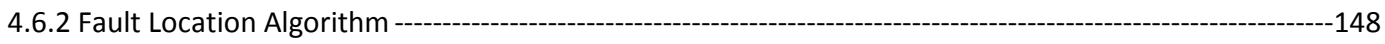

4. 7 Short Circuit Study------------------------------------------------------------------------------------------------- 149

4.8 Fault Zone Location Test cases-------------------------------------------------------------------------------- 151

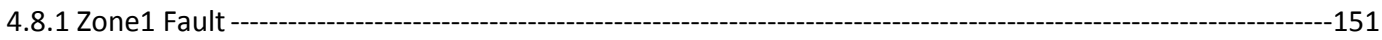

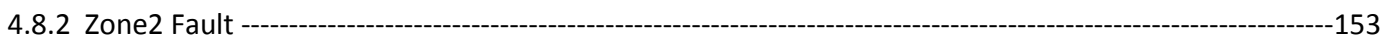

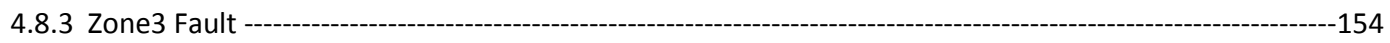

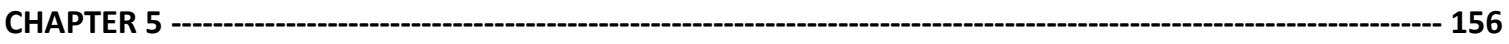

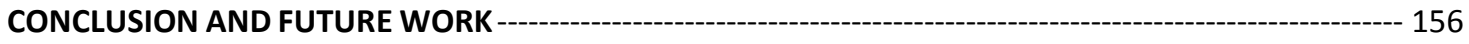

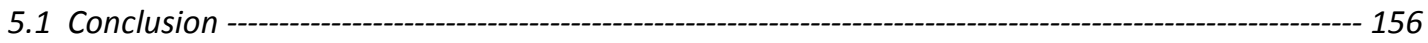

5.2 Future Work ------------------------------------------------------------------------------------------------------------ 156

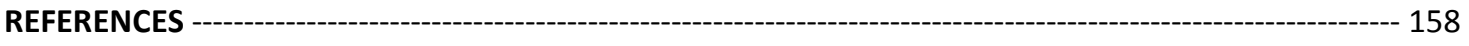




\section{List of Figures}

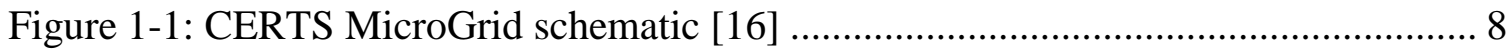

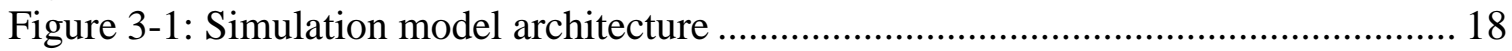

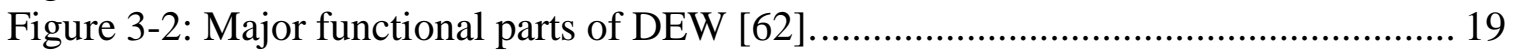

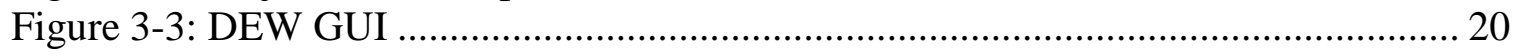

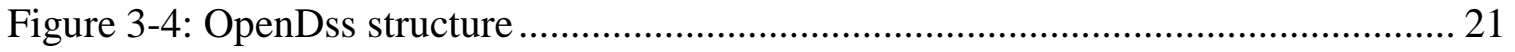

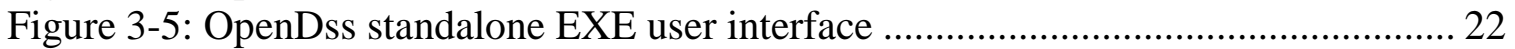

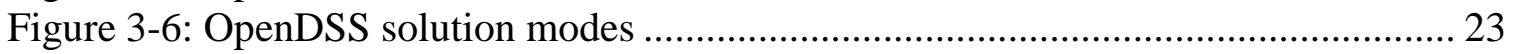

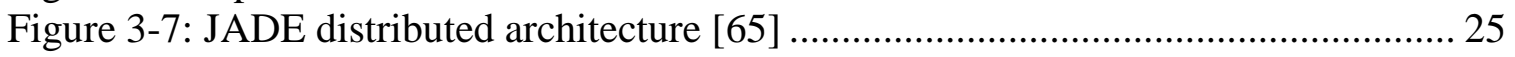

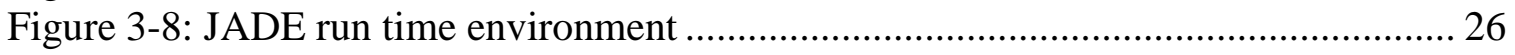

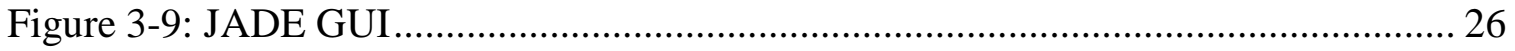

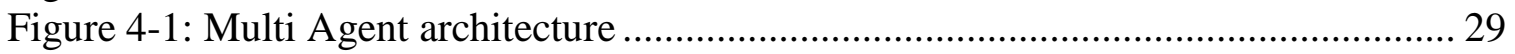

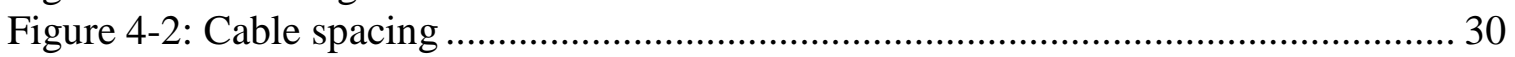

Figure 4-3: Modified IEEE test system ............................................................... 32

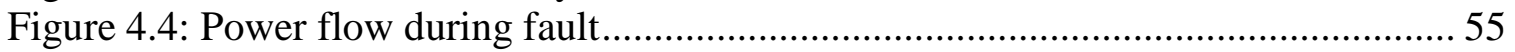

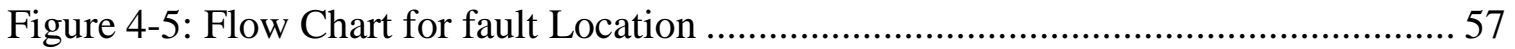

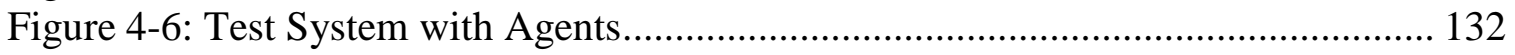

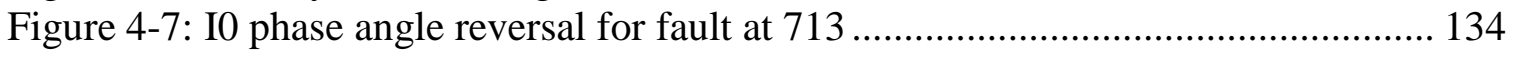

Figure 4-8: JADE Output for fault at 713 Node ...................................................... 134

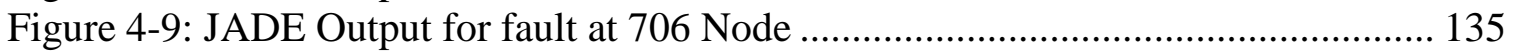

Figure 4-10: Agent Message exchange for Fault Location in Zone1 ........................... 136

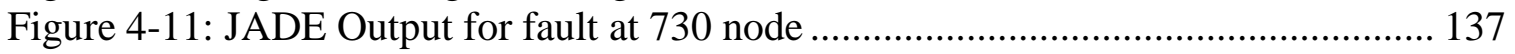

Figure 4-12: I1 phase angle reversal for fault at 730 node ....................................... 137

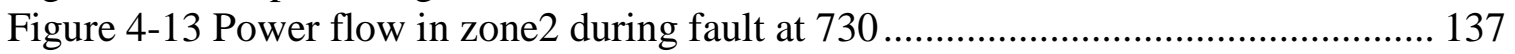

Figure 4-14: $I_{1}$ phase angle reversal for fault at 708 node......................................... 138

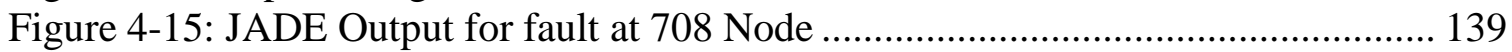

Figure 4-16: Agent Message exchange for Fault Location in zone2 ............................ 139

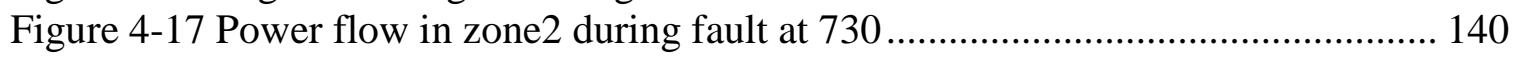

Figure 4-18: JADE Output for fault at 738 Node .................................................... 141

Figure 4-19: Agent Message exchange for Fault Location in Zone3 ........................... 141

Figure 4-20: JADE Output for fault in Zone1 ......................................................... 152

Figure 4-21: Agent Message exchange for Zone1 Fault............................................ 152

Figure 4-22: JADE Output for fault for Zone2 Fault................................................. 153

Figure 4-23: Agent Message exchange for Zone2\&Zone3 Fault ................................. 154

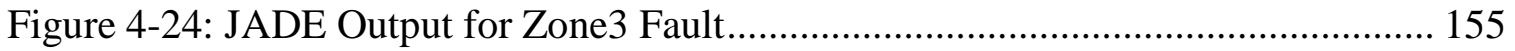




\section{List of Tables}

Table 1-1: FIPA ACL Message Parameters [12] ........................................................... 5

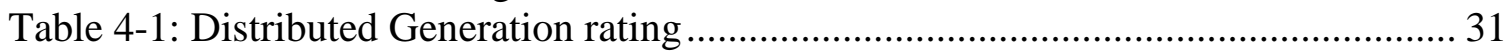

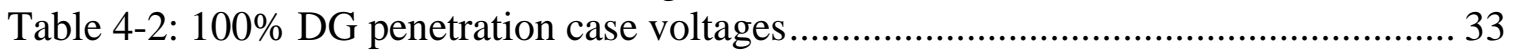

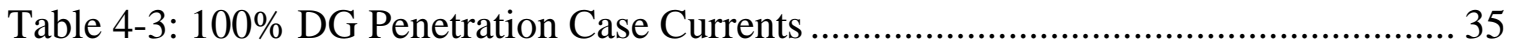

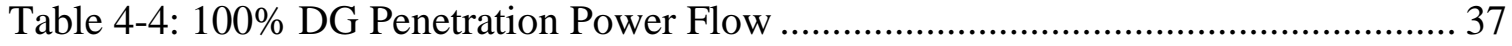

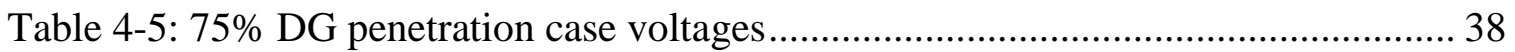

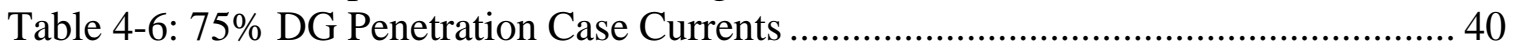

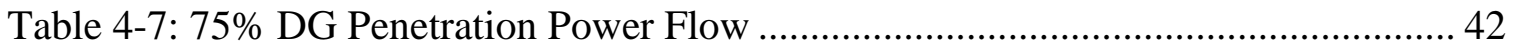

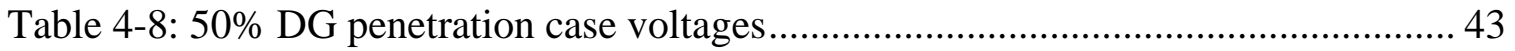

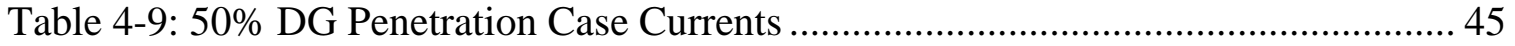

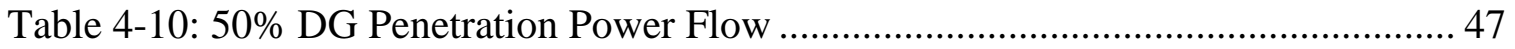

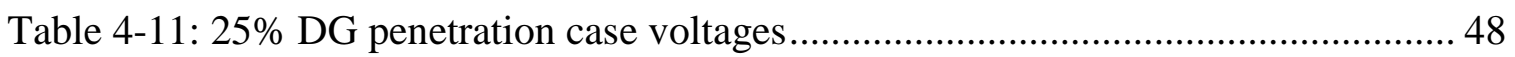

Table 4-12: 25\% DG Penetration Case Currents ......................................................... 50

Table 4-13: 25\% DG Penetration Power Flow ............................................................ 52

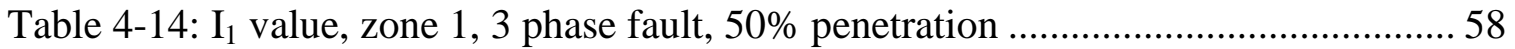

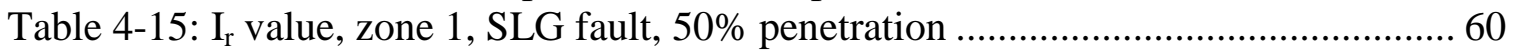

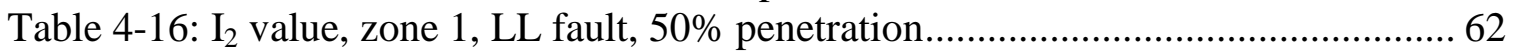

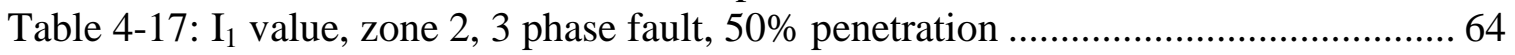

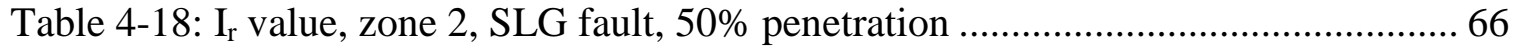

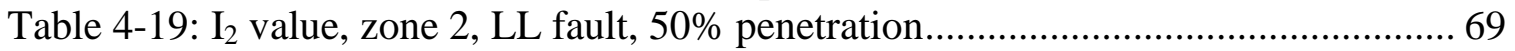

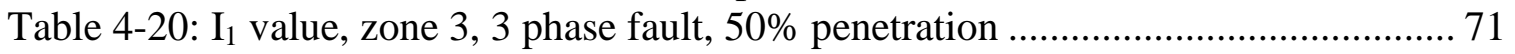

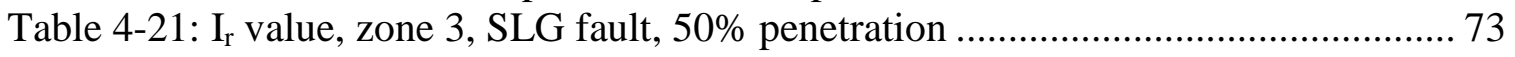

Table 4-22: $\mathrm{I}_{2}$ value, zone 3, LL fault, $50 \%$ penetration........................................... 75

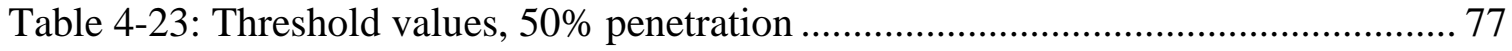

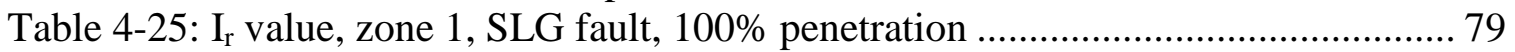

Table 4-26: $\mathrm{I}_{2}$ value, zone 1, LL fault, $100 \%$ penetration............................................ 81

Table 4-27: $\mathrm{I}_{1}$ value, zone 2,3 phase fault, $100 \%$ penetration ..................................... 83

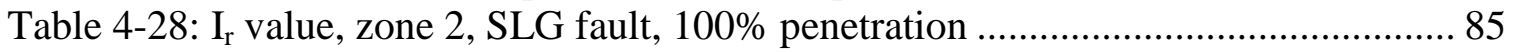

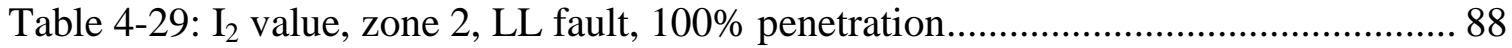

Table 4-30: $\mathrm{I}_{1}$ value, zone 3, 3 phase fault, $100 \%$ penetration ..................................... 90

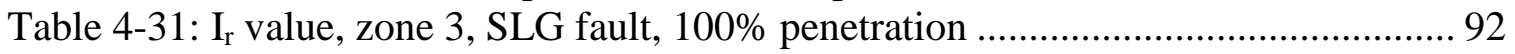

Table 4-32: $\mathrm{I}_{2}$ value, zone 3, LL fault, $100 \%$ penetration............................................ 94

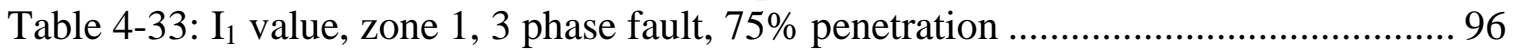

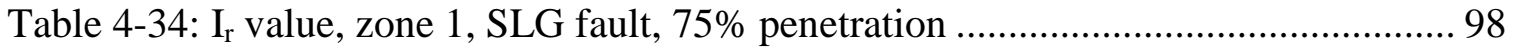

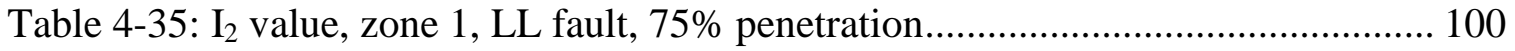

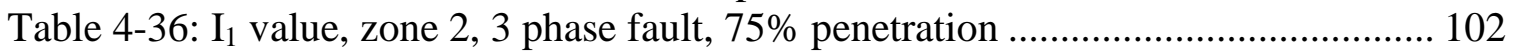

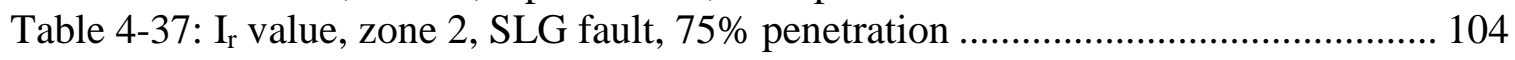

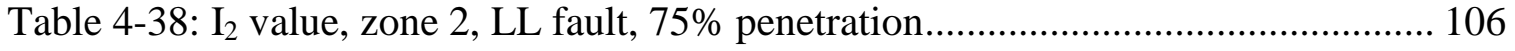

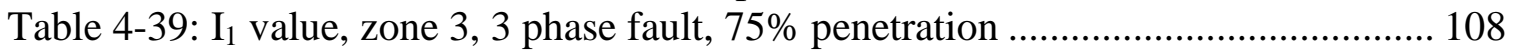

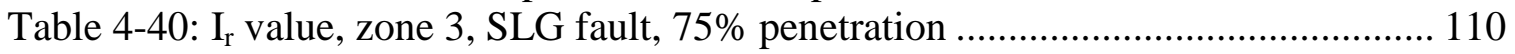

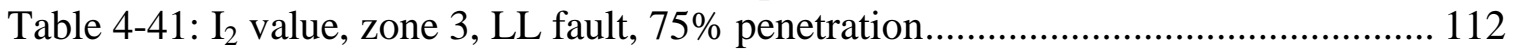

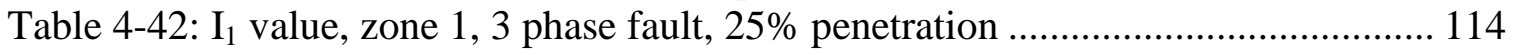

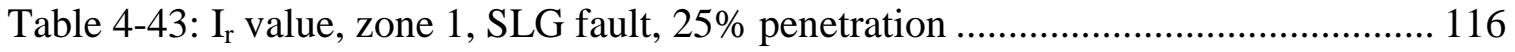

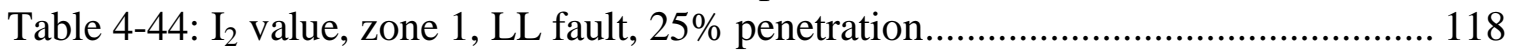




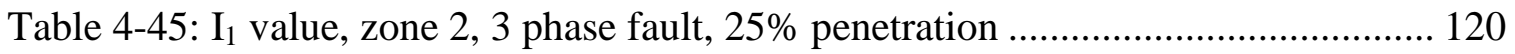

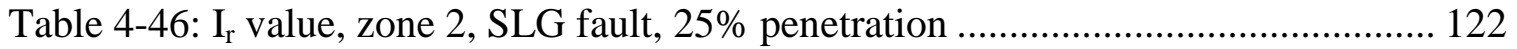

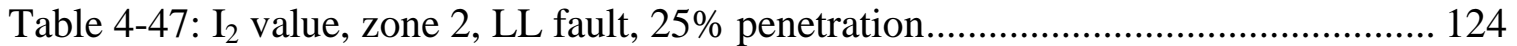

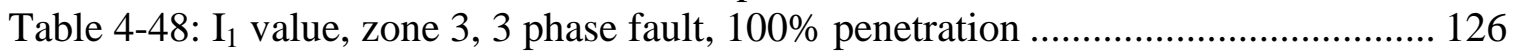

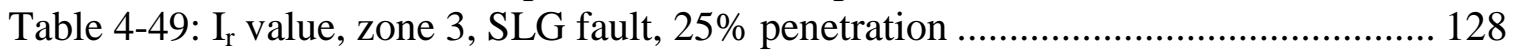

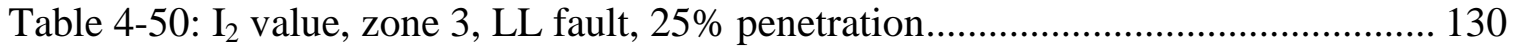

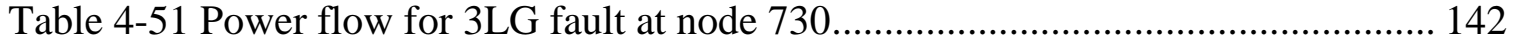

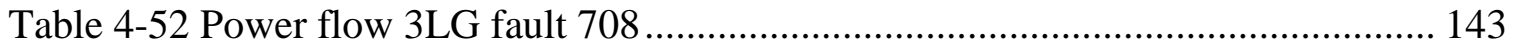

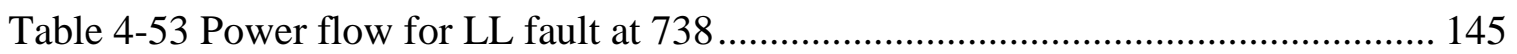

Table 4-54: I1 value for 3LG fault....................................................................... 149

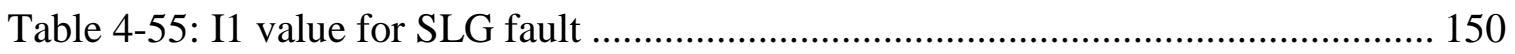

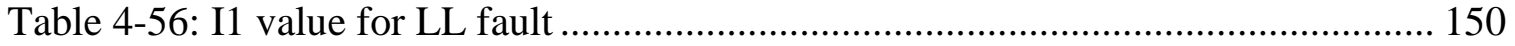

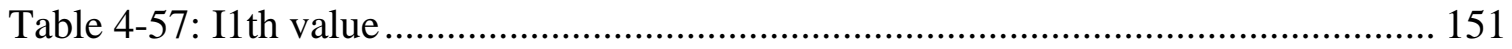




\section{NOMENCLATURE}

ACL Agent Communication Language

DSS Distribution System Simulator

DG Distributed Generation

FIPA Foundation for Intelligent Physical Agents

GUI Graphical User Interface

IEEE Institute of Electrical and Electronics Engineers

JADE Java Agent Development Framework

KQML Knowledge Query and Manipulation Language

MAS Multi-Agent System

OOP Object Oriented Programming

PV Photo Voltaic

WVU West Virginia University 


\section{CHAPTER 1}

\section{INTRODUCTION}

\subsection{Background}

One of the most expensive and complicated system ever developed is the electric power system whose main purpose is to supply electricity to its various consumers. The three main aspects of the electricity supply are generation, transmission and distribution. Initially, all these aspects were integrated and were centrally monitored and controlled. But, with the steep increase in demand for electricity all the resources in these processes are being utilized to the maximum extent possible. So, from the past decade restructuring of the power system, to separately manage the transmission, generation and distribution has been taking place [1].

Apart from restructuring, another aspect that has changed the dynamics of the power system is the introduction of the Distributed Generation at the distribution level. Distributed Generation can be basically defined as a local generation unit at customer level that can be used during a shortage of electricity supply. The power congestion caused due to power system deregulation has been addressed through the installation of Distributed Generation units. The interconnection of Distributed Generation has further complicated the power system. Thus, there is a need to maintain stability, voltage profile and introduction of new protection schemes with Distributed Generation in the system [2].

Though the electric power grid is a huge network, it is highly sensitive to the faults occurring in it. These faults can shut down the power supply to a large area. The main reason for this is the cascading effect that can essentially shut down the whole system. One of the greatest examples is the 2003 northeast blackout that affected nearly 50 million people and caused 6 billion dollars of financial losses [3]. This has triggered research towards increasing the security and robustness of grid and achieving a Smart Grid. Some of the features of the smart grid are self healing, asset optimization, security, 
reliability, demand response etc [4]. One of the initiatives taken in order to improve robustness and security of the grid is the evolution of a Smart MicroGrid, which can supply some critical facilities independently of the main grid. One of the leading technologies to deploy intelligence in the electric grid is the agent technology. The main advantages offered by the agent technology are autonomy and decentralization.

\subsection{Multi Agent Systems}

Agent based system technology has generated lots of excitement in recent years because of its promise as a new paradigm for conceptualizing, designing, and implementing a wide range of applications. These applications range from small scale ones to large industrial applications. This technology has been deployed in the form of Multi Agent systems in a gamut of fields like transportation, logistics, graphics, GIS networking and mobile technologies and now in power system automation as well.

A Multi Agent system can be defined as a congregation of individual intelligent agents that cooperate and coordinate with each other to achieve a common goal. Before dwelling about the important aspects of Multi Agent System it is imperative to understand the basic concept of an agent. Due to the wide range of applications of agent technology, the term 'agent' has been defined in numerous ways. The most appropriate definitions of agent with respect to its implementation in this thesis are listed below.

According to Wooldridge et al. [5], agent is a computer system that is situated in some environment, and is capable of autonomous action in this environment in order to meet its design objectives. While Weiss defines it as an autonomous computational entity such as a software program that can be viewed as perceiving its environment through sensors and acting upon this environment through its effectors[6]. The basic characteristics of an agent according to [7-9] are:

1) Interaction with its environment (which may include other agents) (Sociality)

2) Learning from its environment (Autonomy) 
3) Reaction to its environment in a timely manner (Reactivity and Pro-activity)

4) Taking initiatives to achieve its goals, (Autonomy and Pro-activity)

5) Accomplishing tasks on behalf of its user (Sociality and Reactivity)

In this thesis work an agent is considered as a software entity that can sense a change in its environment and can act meticulously with the sole purpose of accomplishing its design objectives. As described earlier a Multi Agent system is a congregation of these kinds of agents which communicate and coordinate to solve a particular problem according to their objectives.

A critical factor which effects the functioning of an agent based system is its environment. This is because the decisions taken by the Multi Agent system to meet its design objectives are dependent on the environment. In [10] description of the various agent environments possible is provided. They are:

a) Accessible vs. Inaccessible: An accessible environment is one in which the agent can obtain complete, accurate, up-to-date information about the environment's state. Most of the moderately complex systems are in accessible environment

b) Deterministic vs. Non-Deterministic: A Deterministic environment is one in which there is no uncertainty about the state that will result from performing an action. While Non-Deterministic environment is the converse of deterministic. Most of the physical world is Non- deterministic which provides greater problem to the designers.

c) Episodic vs. Non-Episodic: In an episodic environment, the performance of an agent is dependent on a number of discrete episodes, with no link between the performance of an agent in different scenarios.

d) Discrete vs. Continuous: An environment is discrete if it has a fixed, finite number of actions and percepts in it. The best example for a discrete environment is chess game and for a continuous environment is car driving.

e) Static vs. Dynamic: A static environment is one that can be assumed to be unchanged except for the actions by agents. While dynamic environment has many processes operating on it, changing it at every instant. It is also very difficult to control. 
Apart from their environment, another important requirement of Multi Agent system is to successfully perform their designated tasks is the communication between the agents. A brief insight about the communication aspect is provided in section 1.3.

\subsection{Agent Communication}

The pivotal function of the Multi Agent system design in order to accomplish its design objectives is the ability of the individuals to communicate and negotiate with each other. In order to enable communication certain protocols have been specified. These Communication protocols enable agents to exchange and understand messages. The behavior of a Multi Agent system not only depends on its component agents, but also on how they interact. In a Multi Agent system of sufficient complexity, each agent would not only need to do the tasks that arise locally, but would also need to interact effectively with other agents. The communication protocols can be regarded as the specification of these interactions. Protocols are a nice way of enforcing modularity in the design of a Multi Agent system. The most popular languages followed for the Agent Communication are,

- Agent Communication Language (ACL) by the Foundation for Intelligent Physical Agents (FIPA)

- Knowledge Query and Manipulation Language (KQML)

Both these languages, define a set of performatives and their meaning. The content of the performative varies from system to system.

To make agents understand each other, they have to not only speak the same language, but also have a common ontology. Ontology is a part of the agent's knowledge base that describes what kind of things an agent can deal with and how they are related to each other. 


\subsubsection{FIPA -ACL}

FIPA is a body for developing and setting computer software standards for heterogeneous and interacting agents and agent-based systems. It was founded as a Swiss nonprofit organization in 1996 with the ambitious goal of defining a full set of standards for both implementing systems within which agents could execute (agent platforms) and specifying how agents communicate among themselves [11]

The language developed by FIPA for the agent communication is known as Agent Communication Language (ACL). It is based on speech act theory. Here the messages are intended to perform some action by virtue of being sent, hence they can be considered as actions or communicative acts. A FIPA ACL message contains a set of one or more message parameters. The parameters required for effective agent communication will vary according to the situation; the only parameter that is mandatory in all ACL messages is the performative, although it is expected that most ACL messages will also contain sender, receiver and content parameters. However, for a meaningful message there should be other parameters required depending upon the situation. The full set of pre-defined message parameters is shown in the following table.

Table 1-1: FIPA ACL Message Parameters [12].

\begin{tabular}{|l|l|}
\hline $\begin{array}{l}\text { Message } \\
\text { Parameter }\end{array}$ & Meaning: \\
\hline :sender & $\begin{array}{l}\text { Denotes the identity of the sender of the message. i.e., the name of the } \\
\text { agent of communicative act }\end{array}$ \\
\hline :receiver & Denotes the identity of the intended recipients of the message. \\
\hline :content & $\begin{array}{l}\text { Denotes the content of the message; equivalently denotes the object of } \\
\text { the action. The meaning of the content of any ACL message is intended } \\
\text { to be interpreted by the receiver of the message. This is particularly } \\
\text { relevant for instance when referring to referential expressions, whose } \\
\text { interpretation might be different for the sender and the receiver. }\end{array}$ \\
\hline :reply-with & \begin{tabular}{l} 
Introduces an expression that will be used by the responding \\
\hline
\end{tabular}
\end{tabular}




\begin{tabular}{|l|l|}
\hline :in-reply-to & $\begin{array}{l}\text { This parameter indicates that subsequent messages in this conversation } \\
\text { thread are to be directed to the agent named in the reply-to parameter, } \\
\text { instead of to the agent named in the sender parameter }\end{array}$ \\
\hline :encoding & Denotes the specific encoding of the content language expression. \\
\hline :language & Denotes the language in which the content parameter is expressed \\
\hline :ontology & $\begin{array}{l}\text { Denotes the ontology(s) used to give a meaning to the symbols in } \\
\text { the content expression }\end{array}$ \\
\hline :reply-by & $\begin{array}{l}\text { Denotes a time and/or date expression which indicates the latest time by } \\
\text { which the sending agent would like to receive a reply. }\end{array}$ \\
\hline :protocol & $\begin{array}{l}\text { Denotes the interaction protocol that the sending agent is employing } \\
\text { with this ACL message. }\end{array}$ \\
\hline :conversation- \\
id
\end{tabular}

In this thesis work, Multi agent system communication is performed using ACL language. Java Agent Development Framework (JADE) is a Java based agent platform which abides to FIPA standards for agent communication. JADE is recently employed in most of the Multi Agent systems development has been used in this thesis.

\subsubsection{Knowledge Query and Manipulation language}

The Knowledge Query and Manipulation Language (KQML), is a language and protocol for communication among software agents and knowledge-based systems. It was developed in the early 1990s. While originally conceived of as an interface to knowledge based systems, it soon became an Agent communication language.

The KQML message format and protocol can be used to interact with an intelligent systems, either by an application program, or by another intelligent system. KQML's performatives are operations that agents perform on each other's knowledge and goal stores. The basic protocol is defined by the following structure [13]. 
(KQML-performative

$\begin{array}{ll}\text { : Sender } & <\text { word }> \\ \text { : Receiver } & <\text { word }> \\ \text { : Language } & <\text { word }> \\ \text { : Ontology } & \text { <word }> \\ : \text { content } & \text { <expression }> \\ \ldots) & \end{array}$

FIPA - ACL has superseded the KQML in most of the agent communications.

\subsection{MicroGrid}

With the tremendous increase in electricity demand in industrialized countries and the increase in frequency of natural hazards all over the world put forth many difficult challenges of securing critical equipment by seamless and continuous provision of electricity. One such problem occurred in August 2003 when 50 million residents of U.S. and Canada were left without electricity services due to a blackout. Historically, to deal with such situations, more power plants and transmission lines were built to fulfill the electricity requirement. This approach, however, has limitations such as, impacts on carbon emission, fuel availability, land use, health effects due to electromagnetic fields, lack of resiliency etc. Instead, electricity needs for critical equipment could be met by the microgrid concept [14].

MicroGrid can be defined as a low to medium voltage network that contains an aggregation of certain responsive loads and Distributed Generation units, which is connected to the main grid through a point of common coupling. It can operate either in the grid-connected or islanded mode. In the grid connected mode it remains connected to the main grid and is seen as a single aggregate load or source, while in the islanded mode it separates from the main grid, due to a major disturbance, becoming self sustained and continues to serve certain loads [15]. A typical MicroGrid system is shown in Figure 1-1. 


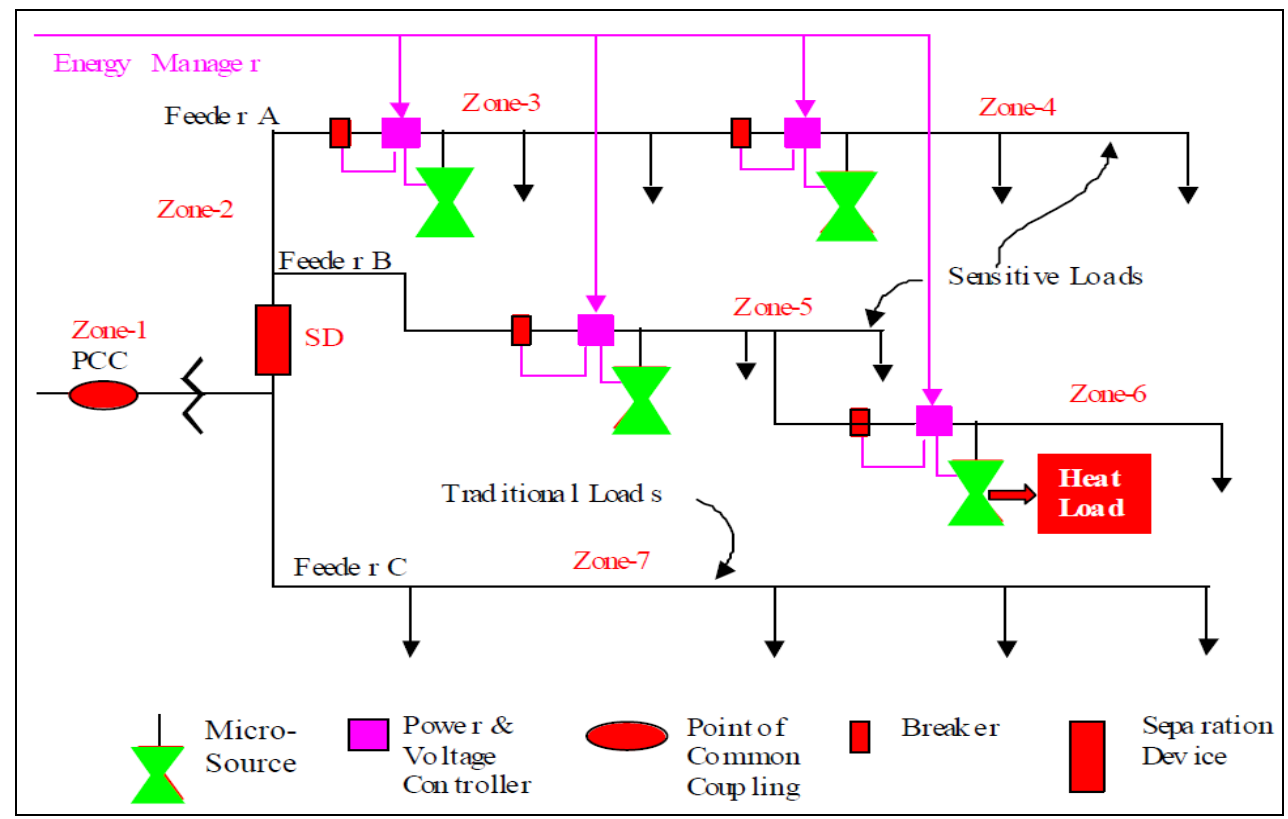

Figure 1-1: CERTS MicroGrid schematic [16]

However, the implementation of a MicroGrid poses several technical difficulties with regard to its protection. The basic problems that crop up with the introduction of Distributed Generation into the Radial distribution system are two way power flow and change in magnitude of the Fault currents. So, in this thesis agent based design for the fault location in Microgrid system has been proposed.

\subsection{Problem Statement}

In this thesis, a Multi Agent system has been implemented to determine the fault location in a smart MicroGrid system. The fault location algorithm implemented in the Multi Agent system is based on sequence current magnitude and direction of the current during a fault. The MicroGrid has been implemented by adding Distributed Generation units at the various nodes of the 37-bus IEEE benchmark system and its fault simulation has been done in the software OpenDss.

The test system considered is divided into 3 zones separated by a switch. Each of these zones has a zone agent and at all the switches separating the zones there is a switch agent. Further, a recloser agent is also present which is located at the recloser near the 
substation. When a fault occurs in the network, these agents communicate among each other and come with the fault location in the circuit. The switch agent has the fault location algorithm embedded in it.

A few assumptions made in this thesis work are listed below,

- The fault location will be performed only when the recloser completely locks out.

- The switch agent has measurement of all the lines in the zone to which it is associated.

- The switch agent can open any line to isolate the faulty line

- The only Distributed Generation source considered for the implementation of the MicroGrid is the PV solar generation

- The default model for PV solar generation available in OpenDss is used.

- Only low impedance faults are considered.

In order to simulate a more realistic scenario, this thesis also implements Multi Agent system based algorithm for locating the faulty zone in the system by using only the measurements at the switches separating the various zones considered in the test system. The only measurement used is the positive sequence current magnitude.

\subsection{Approach}

In order to implement a MicroGrid system, the Radial IEEE 37 bus standard benchmark system was modified by adding PV solar Distributed Generation models at various nodes of the system. The default model for PV solar generation available in OpenDss is used. The circuit has been modeled in open source distribution system simulation software called OpenDSS. The power flow results of OpenDss were verified using Distributed Engineering Workstation (DEW) software. The software used to design the Multi Agent System is Java Agent Development (JADE) framework. The fault detection algorithm was implemented in JADE. Details of each of the software are provided in detail in Chapter 3. 
Interfacing of these two software platforms involved was not necessary since the fault location algorithm is being implemented only after the recloser locks out. Hence the agent application was implemented offline. Different kinds of faults, mainly three phase to ground, single line to ground and line and line fault were created at various nodes in the test system in order to test the working of the algorithm.

\subsection{Thesis Organization}

The rest of the thesis is organized as follows. Chapter 2 primarily provides an extensive review of the research work done in the area of Multi Agent system application for fault location and management of MicroGrid. Further, literature related to Multi Agent system application in other related topics like protection co-ordination, voltage control, Demand response etc. is also discussed. Also, a brief overview of Multi Agent system technology and its advantages are also presented. Chapter 3 elucidates the simulation software packages employed in this work, followed by some insights on their advantages and limitations. Details of the test system, fault location algorithm, Multi Agent system architecture developed for the fault location along with a few test cases are presented in Chapter 4. Conclusions and scope for the future work are discussed in Chapter 5. 


\section{CHAPTER 2}

\section{LITERATURE REVIEW}

This chapter primarily provides a thorough summary of the research work done in the area of Multi Agent system application for fault location and management of Microgrid. Further, literature related to Multi Agent system application in other related topics like protection co-ordination, voltage control, Demand response etc. is also discussed. Along with literature mentioned above, a brief overview of Multi Agent system technology and its advantages and limitations in comparison with the traditional schemes are also presented.

\subsection{Multi Agent System}

The Multi Agent system technology has evolved into being one of the most potent tools in developing a complex system capable of solving difficult problems. It is basically a combination of individual agents that coordinate and cooperate with each other in order to accomplish their assigned tasks which in turn results in achieving the overall goal of the system.

The Multi Agent systems make use of the individual agent characteristics of autonomy, sociality, reactivity and pro-activity. Along with having properties of individual agents the Multi Agent system are designed to be highly decentralized and have an infrastructure to enable communication and interaction between individual agents.

Though the Multi Agent systems have some limitations as discussed in [17], their characteristics provide the perfect solution to the current problems of distributed computing, communication and data integration in power system automation. The Multi Agent systems can offer modular, flexible, and integrated approach for many problems which are of distributed nature in the field of electrical power systems. 


\subsection{Multi Agent System in Automatic Fault Location}

One of the key aspects to ensure reliability and continuous operation of the power system is Fault detection. With the addition of Distributed Generation unit into the power system makes the fault location a more complicated problem because of the introduction of two way power flow. This section provides a brief survey of work done in fault location in a radial power system with and without Distributed Generation.

In [18] the authors propose an agent based fault detection based on fault diagnosis and prognosis in an electric war ship. The future conditions of the system are predicted and agents are trained to act accordingly to take decisions. While, in [19] and [20], the authors propose an automatic fault detection and fault diagnosis mechanism based on several symptoms to differentiate normal and fault conditions. These methods can be based on parameter estimation, parity equations or state observers. Finally, the fault is detected by the use of classification or interference methods. Further, [21] and [22] propose the fault detection mechanism using analytical redundancy. In [23] a decentralized agent based scheme for fault detection in ship board system is discussed.

There has been a lot of work done with regard to fault location in the power system with Distributed Generation integrated into the system. In [24] authors propose an agent based fault location algorithm for a smart distribution system. The agents are located at various node locations and have the local feeder information. When a fault occurs, depending upon the direction and amplitude of the zero transient reactive power flow between neighboring nodes in the distribution test system, the fault location is determined. The zero transient reactive power is calculated by using zero transient voltage and current. But this algorithm is limited only to single line to ground faults.

Zeng Xiangjun et al. [25] propose a decentralized agent based protection which has the capability to detect High Impedance faults, fault location and load shedding for a Distributed Generation system. Faults often produce wide band of frequency energies that are quite different from the harmonics in the normal conditions or switching surges. These fault harmonic energies flow from the fault point to all the generation sources. 
Based on the principle of differential protection, the line with the same direction of fault harmonic energy flow in its two terminals is the faulted line. The proposed agent based protection scheme is developed with current differential relays. Relays as agents cooperate and communicate with each other to locate the fault section and they also take decisions to shed the loads if necessary.

A Multi Agent system based on current magnitude and direction to identify faults in a looped distribution system is proposed in [26]. The circuit is divided into different zones and each zone is separated through a zone breaker where the current measurements are taken. The simple concept used in this paper is that during a fault all the Distributed Generation units must feed the fault because of which the threshold of current set at the various breakers associated with a particular zone are violated. Agents are located at Distributed Generation units and at the zone breakers, which exchange information and locate the faulted zone. This agent system is applicable only to identify zones but not the precise fault location and this algorithm works best when the current thresholds are set high.

In [27] the authors propose a Multi Agent based scheme for fault diagnosis in power distribution networks with Distributed Generators. In this work, relay agents are located such that the distribution network is divided into several sections and they measure the bus currents at their location. Based on the entropy of wavelet coefficients of the measured bus currents measured at the various locations of relays, the type of fault if it is a three line to ground (3LG), single line to ground (LG), double line to ground (DLG) or a line to line (LL) fault is identified. Finally, the fault location is determined.

In [27] the authors present an agent based protection scheme based on wavelet coefficients of the transient fault currents to detect and isolate the faults in a distribution system with high penetration of Distributed Generation [28]. The two aspects of the algorithm are to determine the direction of the fault current and then measure the distance of the actual fault location. The fault current direction is determined based on the sign of wavelet coefficients, while the distance to the faulted point is calculated based on the 
time interval between the travelling waves (and their reflections) arriving from the fault point. Based on the determination of fault direction and location the faulty sections are isolated. In this work relay agents are located such that the distribution network is divided into several sections.

\subsection{Multi Agent system Implementation in Microgrid}

The control and communication within MicroGrids has been implemented in two ways: Hierarchical and decentralized control. Hierarchical control requires a central controller that manages the entire system. The approach is similar to the one used in SCADA. Work done by CERTS [29] is one of the most important in this regard. Decentralized approach is being implemented using agent technology. The Authors in [30-32] have implemented agent based control and communication strategies for the optimal operation of the Microgrid. In [30] Multi Agent system has been designed to increase security and reliability of distributed power resources. Military camp setting consisting of $\mathrm{HQ}$, barracks, mess and hospital having a generator, one critical ,one non-critical load (operating independently) a communal bus connecting the four generators and the agent system consisting of four agents to operate the microgrid were defined.

Multi Agent system implementation controlling a small MicroGrid in its grid connected state is presented in [31]. The main objective of this work was to minimize MicroGrid operational cost. The bids for buying and selling energy in a MicroGrid market were expressed as a symmetrical assignment problem, in which persons are matched with objects and the profit was maximized.

In [32], a Multi Agent system for fault restoration was implemented. The agent architecture is hierarchical in which lower layer agents sense the absence of energy and inform the higher layer agents and the higher layer agents perform the restoration by communicating and cooperating with their peers. Oyarzabal et al. have formulated [33] generic Multi Agent system architecture to be used for MicroGrid management. The main aim was to perform measurements for obtaining data and performing demand side management functions like load shifting, load curtailment and generation scheduling. The main components of the Multi Agent system architecture are MicroGrid central 
controller, MicroGrid source controller (MSC) and load controller (LC, connected to loads). MCC, MSC and LC further consist of several agents performing their specific tasks. Great emphasis of multi agent functionally has given to the secondary regulation system which starts operation when a load change is detected. The change in load makes the batteries modify their power level and the secondary regulation system sets in and assigns the power requirement difference to generator to supply the new load. Further, the authors in [34] present a optimized method to buy energy from the distributed energy resources to meet the demand at lowest possible price. A generic Multi Agent system was presented in [35] as a control framework for Microgrids.

\subsection{Multi Agent system for Restoration and Reconfiguration}

Pioneering work has been done with regard to Multi Agent system implementation for restoration and reconfiguration in a power system after a fault. Many authors reinforce the advantages of decentralized Multi Agent system schemes over the centralized schemes. Brief description of some of the work done is provided in the subsequent paragraphs.

In [36-38] Multi Agent system for the fault location and reconfiguration has been implemented on the Southern California distribution system known as circuit of future. The author [37] proposes a reconfiguration algorithm which tries to supply all the critical loads in the network. When multiple solutions for reconfiguration are available the solution with good voltage profile and least power loss is chosen. Shunt compensation and priority based load shedding is done for improving voltage profile of the system. While in [36] reconfiguration algorithm tries to supply as much load as possible while maintaining the power balance in the circuit along with line constraints. Further, in [38] an artificial immune based Multi agent system for power system reconfiguration is proposed. The algorithm for reconfiguration after locating the fault, tries to serve affected load by taking into consideration the voltage profile, line losses and line capacity constraints. 
In [39] authors propose a highly decentralized Multi agent system architecture for restoration and reconfiguration of a three phase unbalanced distribution system. In this work, all the agents are localized and communicate only to their neighboring agents making the architecture highly decentralized. The restoration is implemented with limits on generation, load priority and transfer capacity of lines. Authors in [40-42], have designed an decentralized Multi Agent system architecture for restoration of bulk power system. Each of these architectures has different layers of agents and each of the agents cooperate and communicate among themselves and also with agents in other layers to come up with the most optimal restoration plan. The results obtained in these works are promising.

\subsection{Multi Agent system in Secondary Voltage Control}

In this section a brief description of the work done with regard to implementation of the Multi-agent system for secondary voltage control in power distribution system is presented.

H.F wang et al. proposes a highly decentralized Multi-agent system design for the purpose of secondary voltage control during system contingencies [43]. In the electrical power system the secondary voltage control is done by the generator Automatic Voltage Regulators (AVRs) in order to improve voltage stability. Each of the voltage control devices like AVR, SVC and STATCOM is an agent. Each of these agents monitor their area for voltage violation and upon sensing the violation, they implement voltage control by switching in their reactive power resources. If the voltage violation is still persists, then the agent will negotiate with its neighbors for reactive power and the neighbor having sufficient reactive power responds.

In [44] an optimally coordinated Multi Agent system design for secondary voltage control is presented. In this agent based scheme, each of the primary and secondary voltage controllers are represented as a set of execution and coordination agent, respectively, who either work independently or coordinate with each other for systemwide voltage control. Under normal conditions, the agent system works as a conventional secondary voltage control system but during contingencies contract net protocol is 
introduced to setup the coordination and cooperation among voltage control agents to eliminate the voltage level violation. The simulation results of the New England 39-bus system show that the proposed control scheme and coordination method are very effective in managing global voltage profile of power system as well as settling local voltage violation problems during system contingencies.

\subsection{Multi Agent system other Applications}

Apart from the applications discussed in sections 2.2 to 2.5 Multi Agent systems have also been used for other power system applications like protection coordination [45-50], power visualization [51], demand response [52-58]. Apart from power system applications Multi agent systems have also been employed in other engineering fields for highway traffic management control systems [59], air traffic control system and mine detection [60]. 


\section{CHAPTER 3}

\section{SIMULATION SOFTWARE}

This chapter briefly discusses about the various software packages that have been used in this thesis to simulate the MicroGrid and develop the Multi Agent System for fault location.

One of the biggest challenges in this thesis was the selection of the appropriate software that could simulate the distribution test system and the Multi Agent system. The distribution system modeling software must not only perform a 3-phase unbalanced power flow, fault analysis of distribution system but must be able support the islanded Microgrid implementation. Some of the commercially and open source distribution system modeling software are DEW, OpenDSS, Cymdist, Milsoft Windmil and RDAP. Keeping in mind all the requirements of the software, OpenDSS was chosen while Distributed Engineering Workstation (DEW) was used to verify the power flow results obtained in OpenDss. Further, Java Agent Development Framework (JADE) software has been used to develop the agent model in this thesis work.

The simulation model for this work consists of two layers a) Agent model layer and b) Power distribution system software layer. Figure 3-1 depicts the interaction between the two layers through an Interface. In this work no interface has been developed but agent layer acts on the values exported from the distribution system software layer to an excel file in a comma separated values (csv) format.

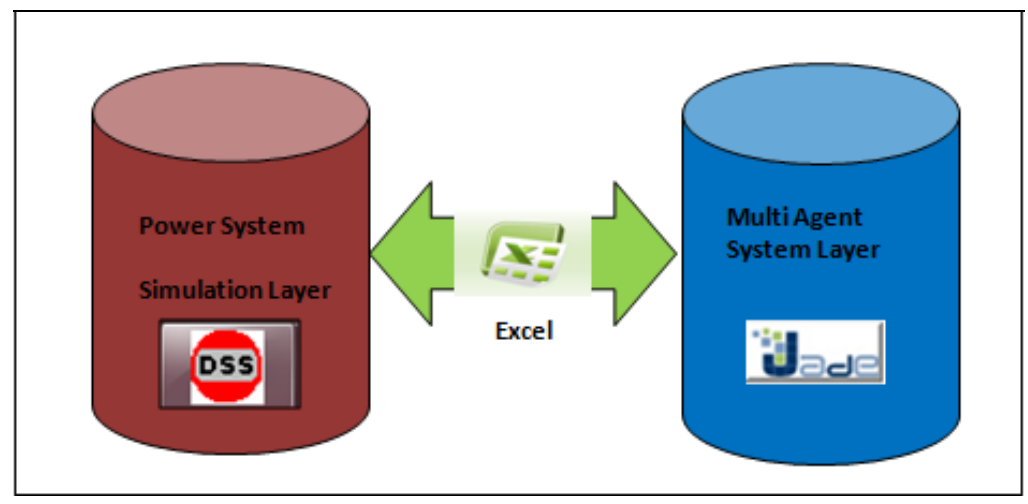

Figure 3-1: Simulation model architecture 


\subsection{Distributed Engineering Workstation@ (DEW)}

Distributed Engineering Workstation is a distribution system modeling software which provides multidiscipline, open-architecture environment with integrated data and applications. It was developed by Electrical Distribution Design Inc. It is capable of meeting the analysis, planning, design, and operation needs of today's distribution systems. DEW offers a wide gamut of applications related to power distribution system like power flow, contingency analysis, Load forecasting, fault analysis, protection coordination design, phase balancing and many more [61]. DEW also has provisions for add-ons that can be separately bought from its providers. The open architecture of DEW allows the integration of external applications into the workstation.

The pivotal components of DEW are the GUI, API, Executive, Database and Application module [62]. All these components have been depicted in Figure 3-2.

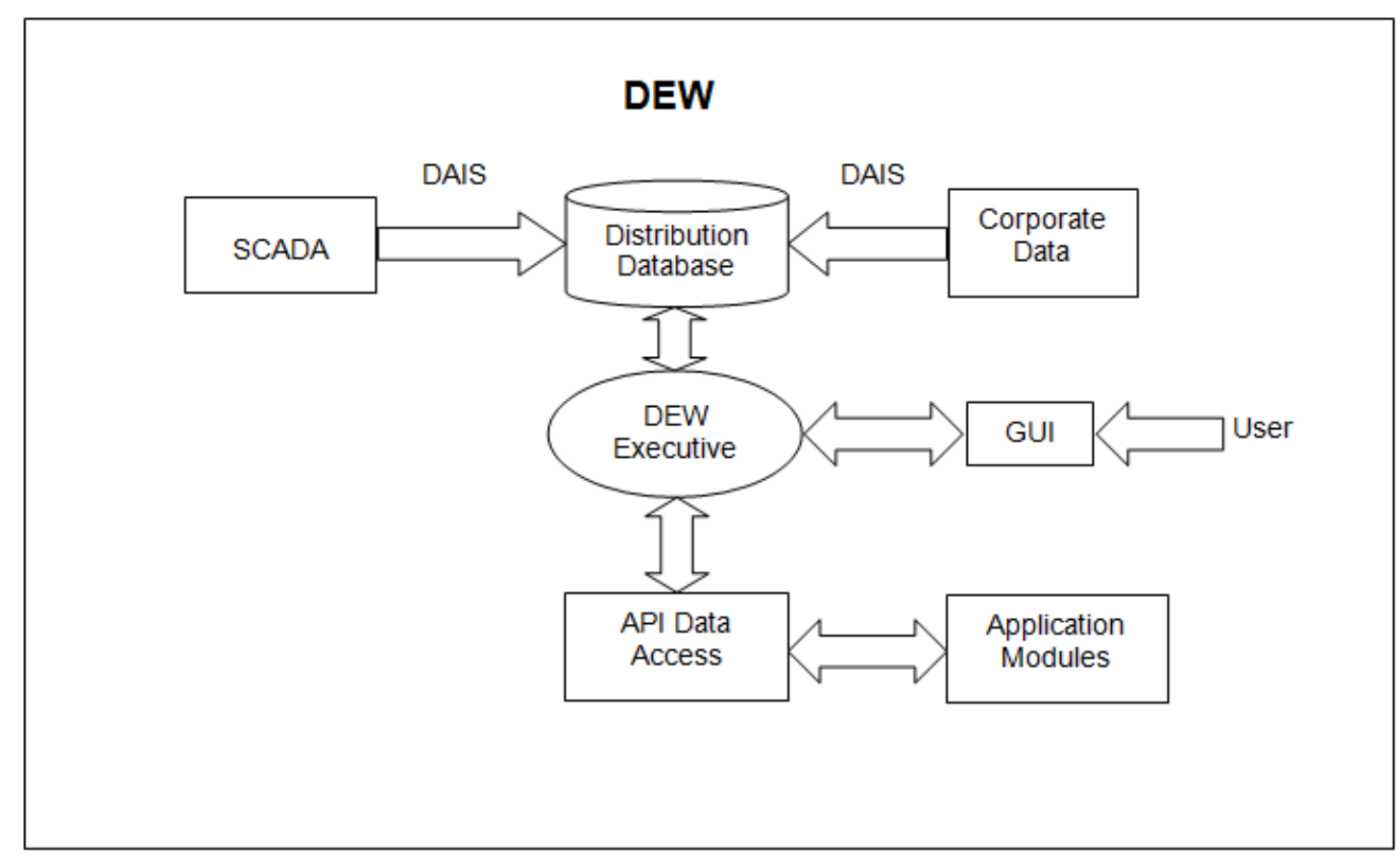

Figure 3-2: Major functional parts of DEW [62]. 
Executive unit handles the key operations in the workstation. It serves as an interface between API, GUI, and database modules. The main function of the API is to amalgamate the application modules into the framework smoothly without disturbing other parts of the workstation. Dew GUI provides user interaction and display functions for all of the remaining DEW applications. All the DEW applications have ready accessibility to the data. Each of the applications utilize data from the SCADA system through an interface called Database Access Integration Service (DAIS)

Figure 3-3 shows the GUI of DEW. To build the single line model of the required distribution system the different power system components available on right hand side pane have to be dragged on to the workspace located to the left of the pane. In this thesis work DEW has been used to validate the power flow results obtained from OpenDss by running a base case test system.

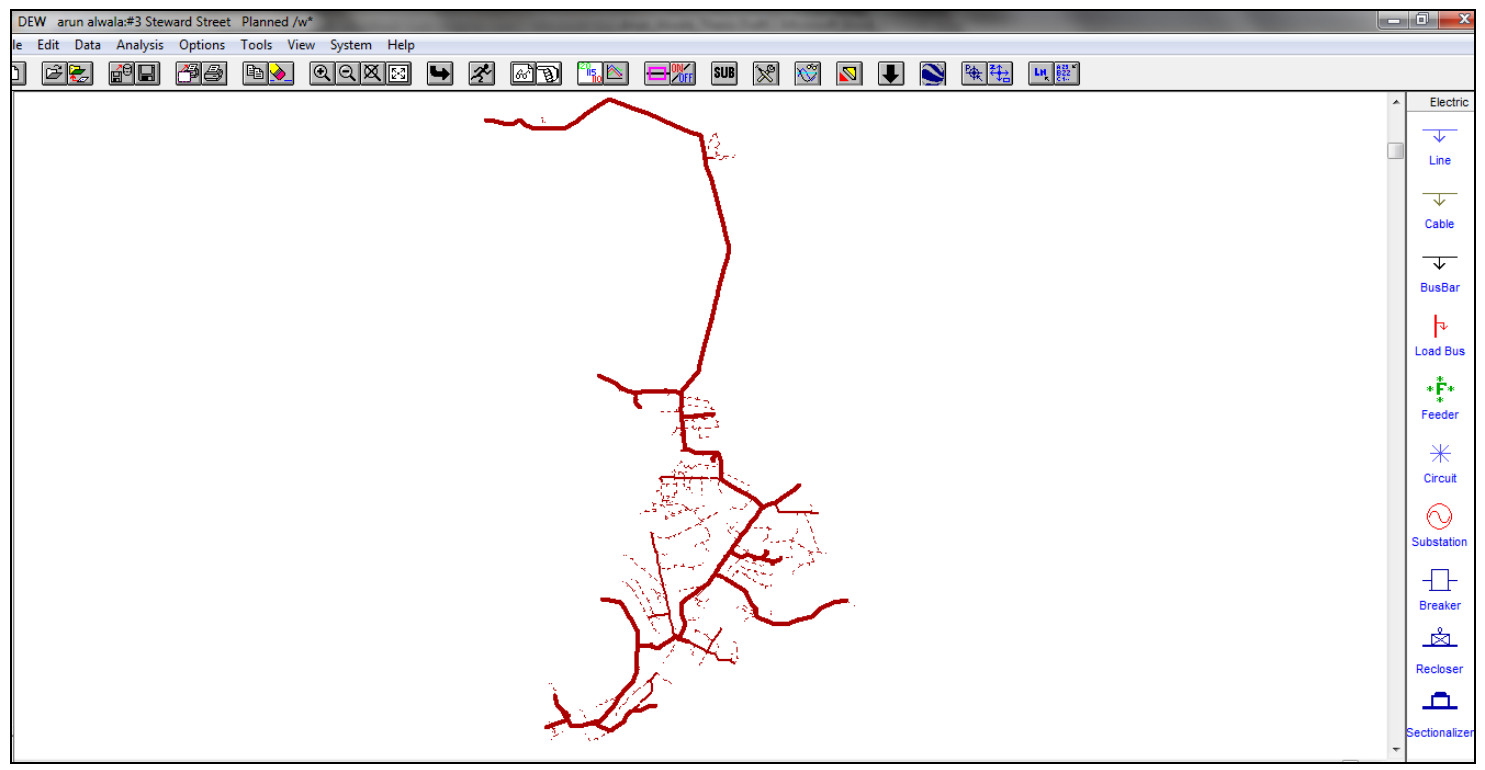

Figure 3-3: DEW GUI

\subsection{Open Distribution System Simulator (OpenDSS)}

OpenDSS is an open source comprehensive electric power Distribution System Simulator (DSS) for supporting distributed resource integration and grid modernization efforts developed by Electric Power Research Institute (EPRI) [63]. The DSS supports all 
steady-state mainly frequency domain analyses commonly performed on electric power distribution systems, such as power flow, harmonic analysis and fault studies. Further, DSS provides an adaptable research platform and foundation for special distribution system analysis like Distributed Generation (DG) interconnection analysis and provides features supporting the enhanced study of energy efficiency, stray voltages, and distribution state estimation.

The DSS can be basically implemented in three ways 1) Standalone EXE user Interface 2) COM interface and 3) User written DLL. In the standalone EXE user interface the users are provided with multiple-windows to assist in building customized distribution systems and solving them. The COM interface can be used to drive the simulator from compatible softwares like MATLAB, VBA, C\#, Python and so on. Expansion of DSS capabilities is made possible by the incorporation of sophisticated models of distribution system through user written DLL'S. These models can be linked to be DSS engine for implementation purposes. The DSS structure constituting these different implementations is shown in Figure 3-4 [64].

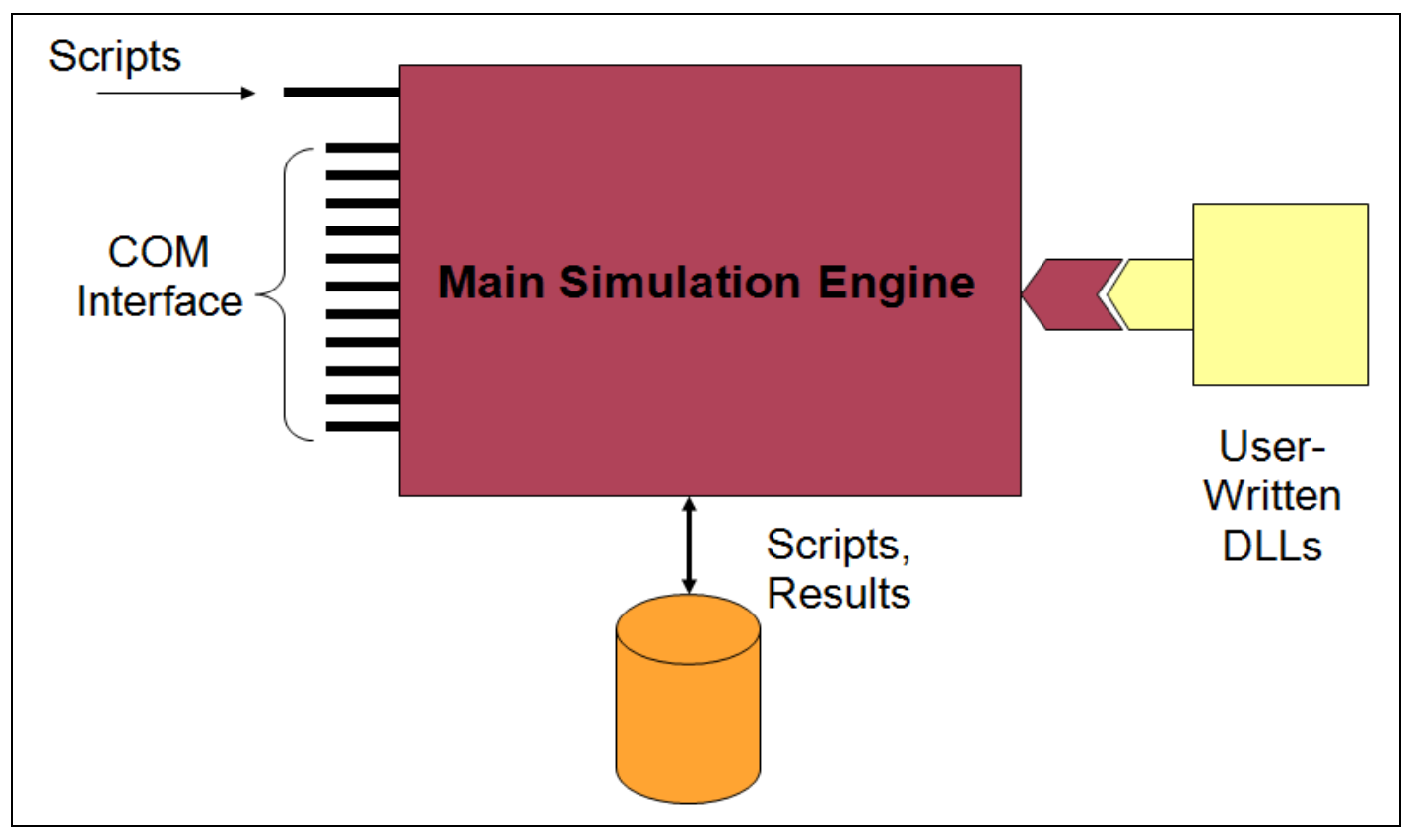

Figure 3-4: OpenDss structure 


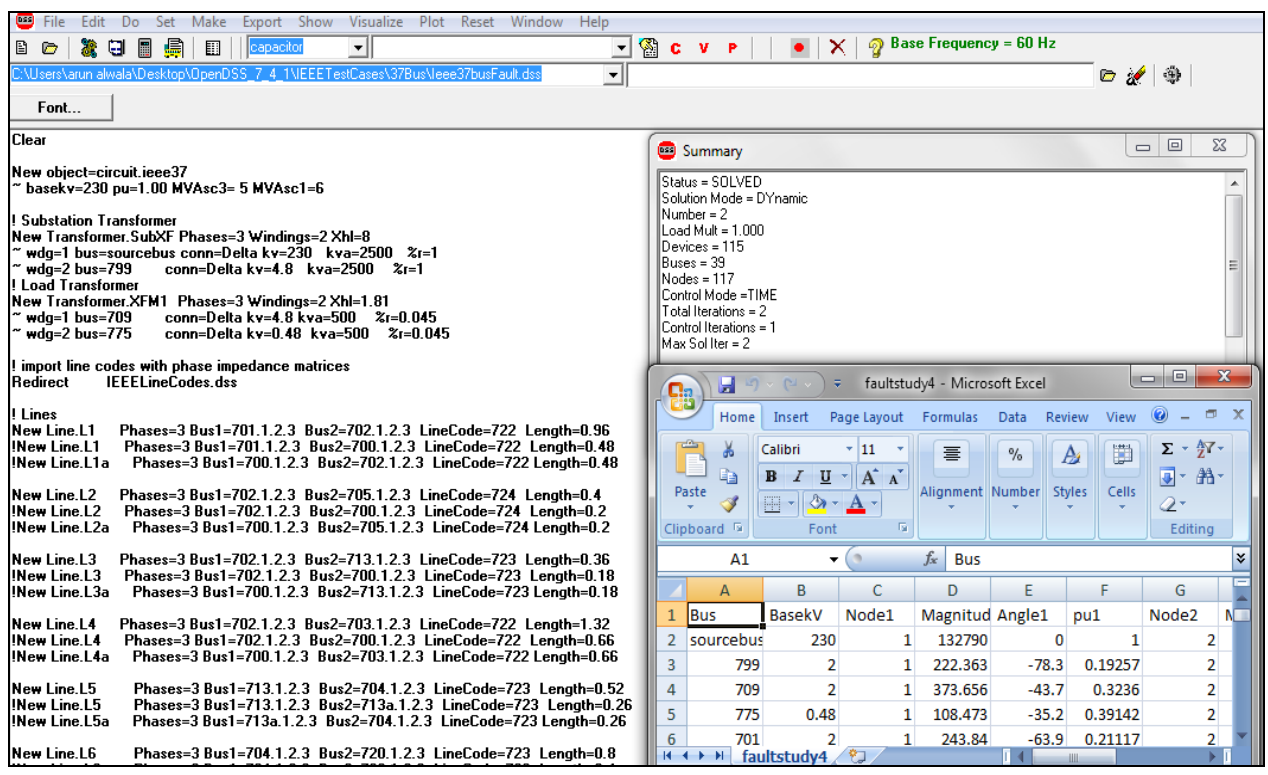

Figure 3-5: OpenDss standalone EXE user interface

\subsubsection{DSS Solution Modes}

DSS offers different types of solution modes which depending on requirement can be used. These different modes have been shown in Figure 3-6. As shown in the figure the different modes are:

- Normal Power flow: This is a default mode in DSS which solves a normal distribution power flow in which the bulk power system is the dominant source of energy. It can solve networked (meshed) and radial systems along with the ability to perform transmission type power flow on small to medium size networks. In this mode there are two types 1) Snapshot in which a single power solution is solved for a given load using iterative methods and 2) Direct in which single power flow solution can be obtained without any iterations.

- Dynamic power flow: This is the mode which supports time series distribution analysis in which power flow solution can be obtained for a load shape defined for a year or day. For different types of dynamic power flow load shapes vary. Load shapes can be imported from CSV file or can be written in the script file in the form of multipliers. In the Daily mode power flow solution is done for each hour in a day for a daily load shape. Similarly, in the yearly mode, yearly power flow simulation can also solve for a Yearly load shape. 
- Dynamics: In this mode DSS can perform basic machine dynamics simulations modeling that can be used to evaluate DG interconnections. Further, fault simulation is mostly done in the dynamics mode. Thus the simulation of the distribution system which involves both DG and fault simulation this mode has been used to run the solution Mode

- Harmonics: This is one of the major solution types of OpenDSS which is used for analyzing harmonic effects of wind turbines and inverter based PV on voltage regulators and capacitor switching in distribution system.

- Fault studies: A fault in OpenDss is often simulated in dynamics mode. There are three types of fault studies that can be done 1) Conventional fault study in which currents and voltages at all the buses for different kinds of faults are calculated 2) Monte Carlo fault study in which faults predefined by user are randomly applied and 3) A single snapshot fault study in which user places a fault on the system at a particular bus, defining the type of fault and the value of the fault resistance. In this thesis work, this mode of the fault study was used to define different kinds of faults like Single line to ground (SLG), Line to Line (LL) and three phase to ground fault were defined at a single instant.

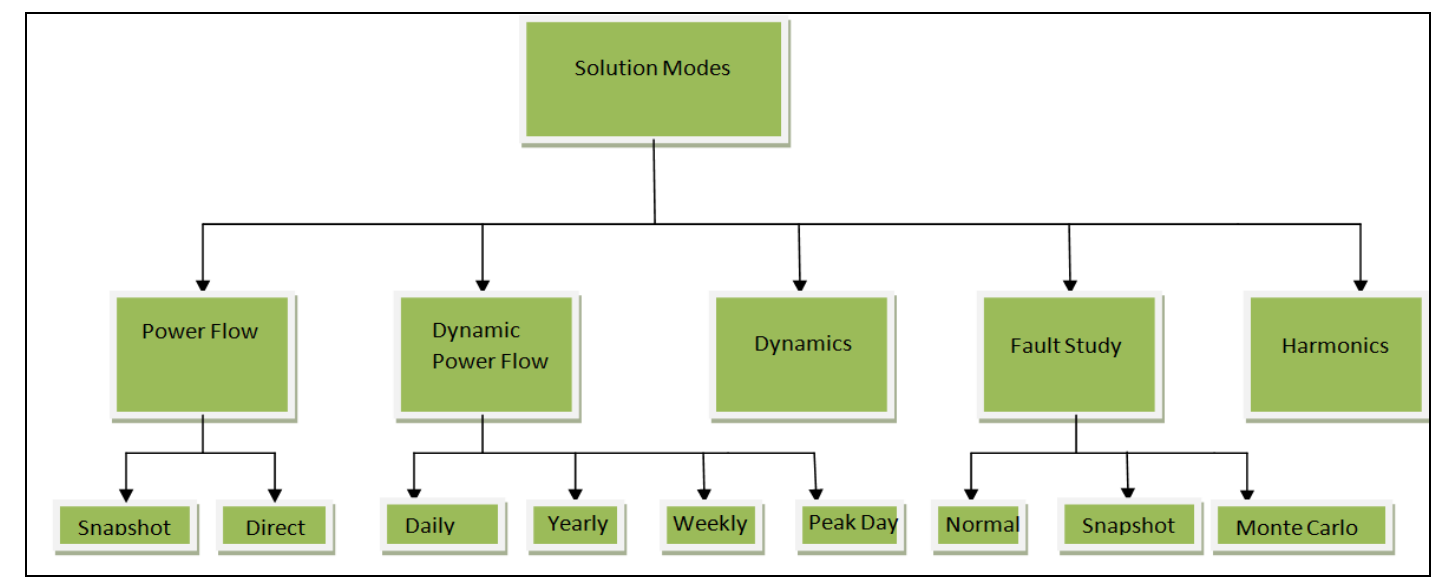

Figure 3-6: OpenDSS solution modes

\subsection{Java Agent Development Framework (JADE)}


JADE is a Java based software framework used to develop agent applications in compliance with the FIPA specifications for interoperable intelligent Multi Agent systems [65]. JADE can also be considered an middle-ware that implements an agent platform and a development framework. It primarily provides the agent and behavior abstractions, transparent distribution of agents across a wide range of devices, peer-topeer communication between agents and publish-subscribe discovery mechanisms that allow agents to find each other. Further, it also has a number of additional features such as agent mobility, ontologies and content language support, fault tolerance and web services integration and a rich suite of graphical tools that facilitate the administration of a JADE based application.

$\mathrm{JADE}$ is one of the most widely used agent development frameworks. The $\mathrm{R}$ and $\mathrm{D}$ wing of Telecom Italia JADE was initially developed in 1998 by the Research and Development department of Telecom Italia s.p.a., but in 2000, it became open source as a community project and was distributed as open source under the LGPL (Library Gnu Public License). JADE can be freely downloaded from Telecom Italia website [65], with documentation, examples and other important information.

\subsubsection{JADE Architecture}

The two important aspects of the JADE architecture are the libraries of java classes required to develop application agents and the run-time environment that provides the basic services and must be active on the device or the network for agent instantiation and execution. Each instance of the JADE run-time is called container in which all the agents are embedded. Some of the important libraries of JADE are jade.core, jade.lang.acl, jade.domain, jade.content etc. The run time environment in JADE is provided by the Main container which is launched first and the other possible containers must join by registering with it. The functions of the Main container are creation and management of container table and global Agent descriptor table which provide registry for agent object references, transport addresses, current status and location. Further, the main container also contains of Agent Management System (AMS) and the Directory Facilitator (DF) agent which are used for agent management and provide white and yellow page service. 


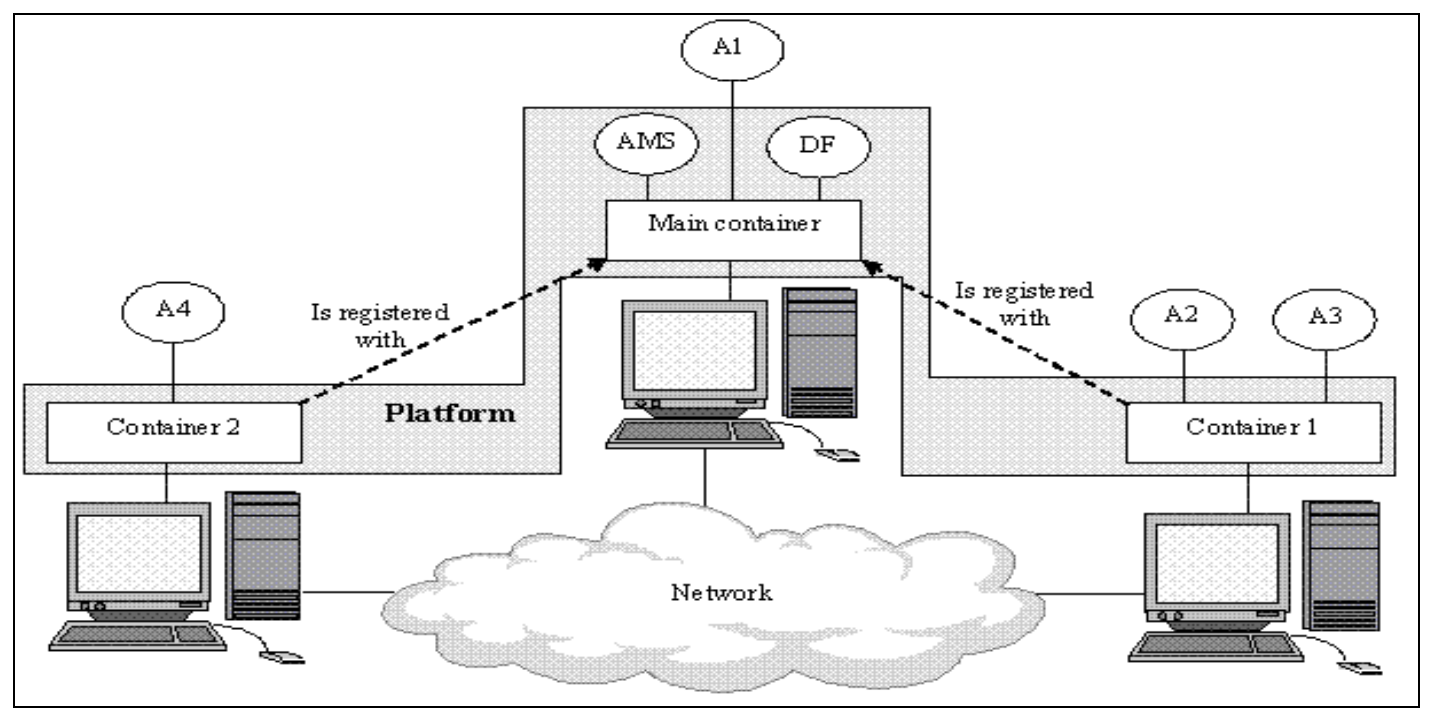

Figure 3-7: JADE distributed architecture [65]

Apart from AMS and DF agent there are many agents available in Jade for the development and debugging of agent platforms. These agents are Dummy Agent, Sniffer Agent, and Introspector Agent. The Dummy Agent is used to simulate other agents behavior by sending ACL messages to them and analyzing the received messages. The sniffer agent can be used to visualize the message exchange between agents which is really helpful for debugging purposes. While the Introspector Agent is useful to control and monitor agent sent and received messages, scheduled behaviors of agent, and the agent life cycle. Thus is useful to debug Agents.

Figure3-8 shows the output of JADE run-time environment, when JADE is launched. While Figure 3-9 represents the Jade GUI. 


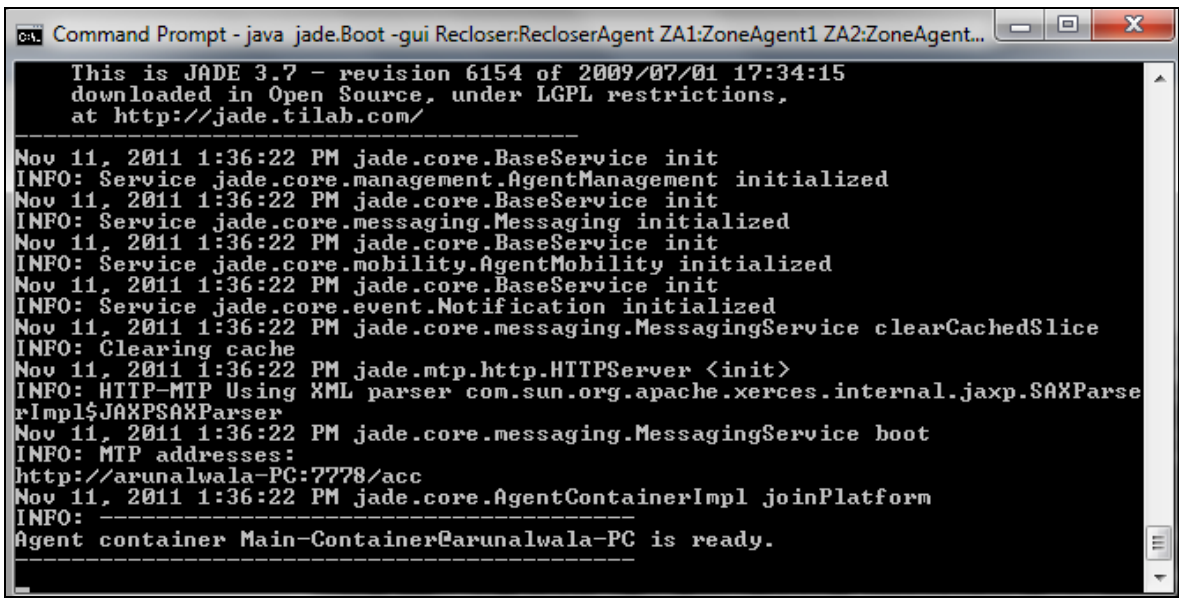

Figure 3-8: JADE run time environment

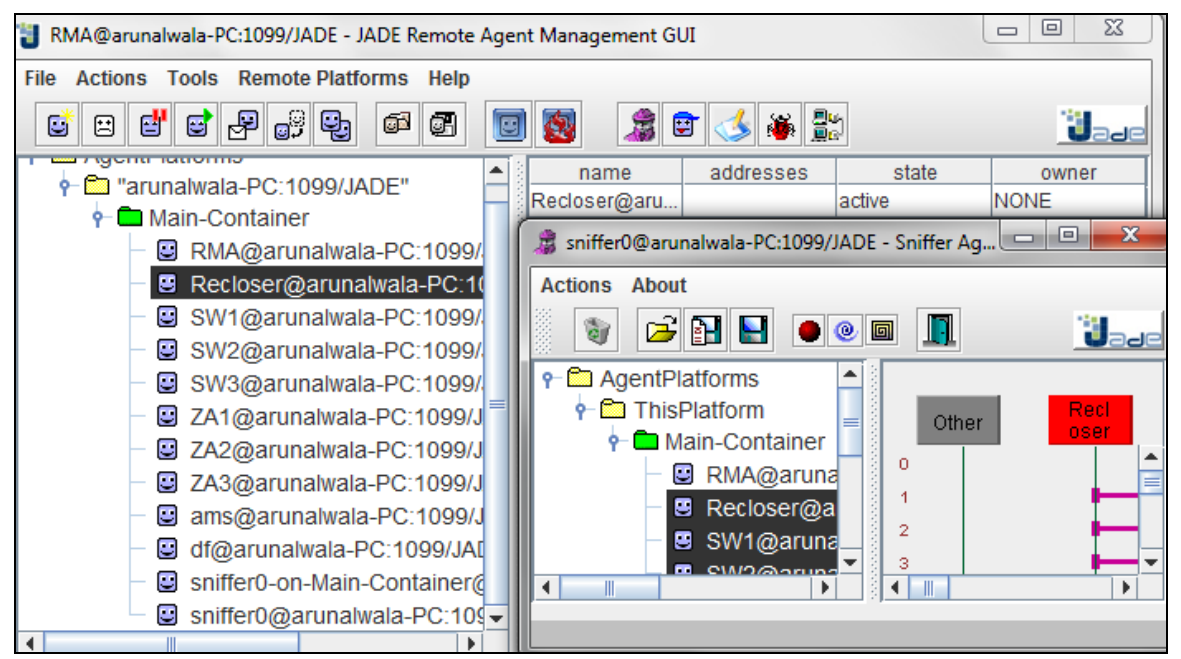

Figure 3-9: JADE GUI

\subsubsection{JADE Programming}

As mentioned in section 3.3 JADE is a java based platform and thus programming in it involves usage of java concepts and syntax. Every agent that is created must extend the agent class defined in the Library jade.core.agent and the agent implementation for initialization purposes has to be done in the setup method. Another aspect of agent creation is the usage of the appropriate 'behavior' which defines the typical operations to be performed by the agent. Some of the behaviors available in JADE are cyclic behavior, 
simple behavior and general behavior. In the creation of agents for this work simple behavior has been employed for all the agents.

Every agent that is created is identified by a unique Agent identifier (AID). To retrieve the AID of a particular agent get $\operatorname{AID}()$ method of jade.core.AID is used. Agent names are of the form <local-name>@<platform-name> and every agent must have a unique name globally.

The most pivotal aspect of a Multi Agent system is communication which is also a fundamental feature in JADE. All the messages between the agents are asynchronous and are based on ACL language that is FIPA compliant. All the messages received by an agent are stored in its message queue. The send() and receive () methods are used by an agent to send and receive messages. In order to terminate an agent doDelete() method is used.

JADE has been used in this thesis work for developing the Multi agent system for the Modified IEEE 37 bus Microgrid test system. 


\section{CHAPTER 4}

\section{FAULT LOCATION ALGORITHM AND RESULTS}

In this chapter, the fault detection algorithm, test system, agent architecture and test cases are presented. The fault detection algorithm is developed in JADE. For modeling and designing the test system, OpenDSS has been used.

\subsection{Multi Agent System Architecture}

The proposed Multi Agent system architecture for fault location is illustrated in the Figure 4-1. According to the architecture, there are mainly two categories of agents available:

- Global Agent

- Zonal Agents

Recloser Agent (RA) is a Global Agent that keeps track of the status of the recloser at the substation and informs the Zone Agents in various zones when the recloser locks out. The Zone Agent (ZA) and Switch Agent (SW) fall into the category of Zonal Agents. These agents are responsible for managing each zone in the power distribution system considered in Figure 4-3. Each Zone Agent has an associated Switch Agent which is responsible for implementing the fault location algorithm.

When a permanent fault occurs in the system, the recloser tries multiple times and locks out, upon which the Recloser Agent informs the Zone Agents about the lock out. Then, the Zone Agent informs its corresponding Switch Agent to check for a fault and implement the fault location algorithm, described in section 4.3. The Zonal Agents have local information like power flow, sequence current magnitude and phase angle of all the distribution lines in their respective zones.

In view of the real world deployment of these agents, information regarding sequence current magnitudes and phase angles can be obtained from the real world protective 
relays described in [65-68]. Zonal Agents implement fault location in a decentralized manner. So, the proposed Multi Agent architecture is decentralized.

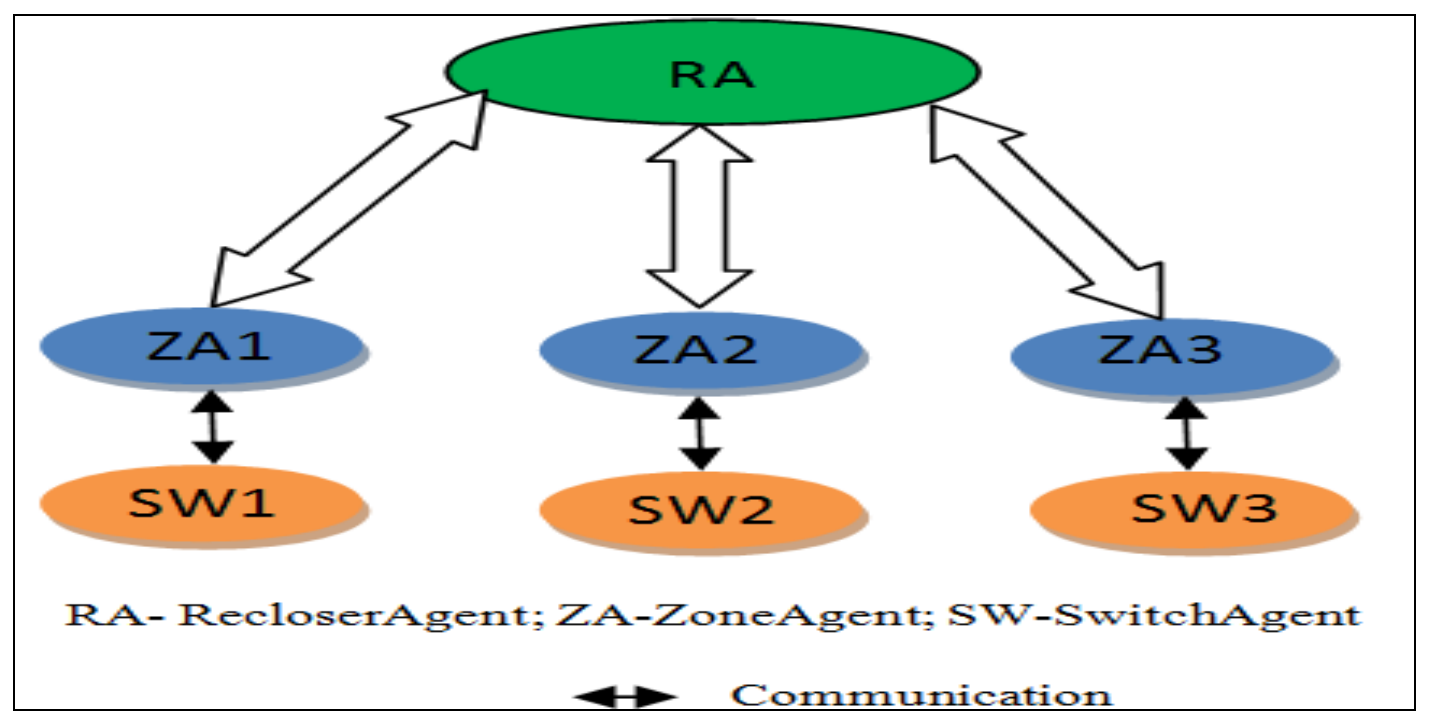

Figure 4-1: Multi Agent architecture

The main responsibility of each agent is described below:

- Recloser Agent (RA): This agent keeps track of the status of the recloser at the substation and informs the Zone Agents in various zones when the recloser locks out. After the lock out, the tripping off action of the recloser has been implemented by opening the substation transformer in the test system. So, the Recloser Agent sees negligible current in all phases of the substation transformer that has been opened and informs the Zone Agents about the lock out.

- Zone Agent (ZA): This agent informs its corresponding Switch Agent about the recloser lock out and asks it to implement the fault location algorithm. The Zone Agent receives the fault location from the Switch Agent after it locates the fault.

- Switch Agent (SW): This agent is the most important entity in this agent architecture, since it implements the fault location algorithm. The Switch Agent has information of the power flows, sequence current magnitudes and phase 
angles of all the lines in its zone. The values of sequence current components are obtained directly from the simulation of the test system in OpenDSS software. The fault location algorithm embedded in the Switch Agent is described in section 4.3 .

\subsection{Test System}

The IEEE 37 bus system was selected as the system of choice for this research. It represents an actual underground radial distribution feeder in California operating at $4.8 \mathrm{kV}$ under heavy phase imbalances [69]. It is configured as a three-wire delta system and therefore has delta connected loads only. It does, however, have many different lineto-line loads that contribute to its unbalanced conditions. In addition, they are buried underground with a Spacing ID of 515, corresponding to configuration in the following figure:

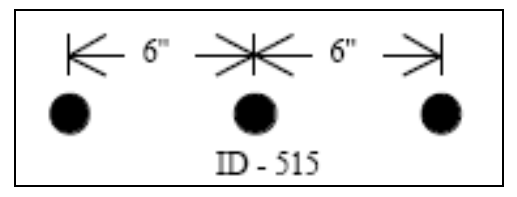

Figure 4-2: Cable spacing

To achieve the MicroGrid, the standard IEEE 37 bus system was modified by adding Distribution Generation units at various nodes in the circuit. Distributed Generation unit considered here is solar. The default PV model of the solar generation available in OpenDSS was used in the simulation. The capacity of these Distributed Generation units depends on their penetration level in the MicroGrid. The penetration levels considered are $25 \%, 50 \%, 75 \%$ and $100 \%$. The details of the rating of the generation units for these various penetration levels are provided in Table 4-1.

In order to implement the Multi agent system described in section 4.1, the Modified IEEE 37 bus system has been divided into three zones such that the Distributed Generation units in that zone can supply either a part or the full load in that zone. The Zone1 considered has 15 distribution lines (L1-L15), 9 loads demanding $779 \mathrm{KW}$ and $344 \mathrm{KVar}$ 
and 3 Solar Distributed Generation units connected at nodes 718,724 and 742. While, Zone2 has 10 distribution lines (L16-L25), 8 loads demanding $549 \mathrm{KW}$ and $267 \mathrm{KVar}$ and 2 Distributed Generation units of solar at 729 and 731. Then, Zone3 has 9 distribution lines (L26-L34), 7 loads demanding $567 \mathrm{KW}$ and $275 \mathrm{KVar}$ and 2 Distributed Generation units at 736 and 741. The modified IEEE 37 bus system is shown in Figure 4-3.

Table 4-1: Distributed Generation rating

\begin{tabular}{|c|c|c|c|}
\hline $\begin{array}{c}\text { Penetration } \\
\text { Level }\end{array}$ & Zone1Generation & Zone2Generation & Zone3Generation \\
\hline $100 \%$ & $\begin{array}{l}\mathrm{DG}_{724} 260 \mathrm{kw} \text { p.f } 1.0 \\
\mathrm{DG}_{718} 260 \mathrm{kw} \text { p.f } 1.0 \\
\mathrm{DG}_{742} 262 \mathrm{kw} \text { p.f } 0.9\end{array}$ & $\begin{array}{l}\mathrm{DG}_{729} 275.0 \mathrm{kw} \text { p.f } 1.0 \\
\mathrm{DG}_{731} 274.0 \mathrm{kw} \text { p.f } 0.9\end{array}$ & $\begin{array}{l}\mathrm{DG}_{736} 280 \mathrm{kw} \text { p.f } 1.0 \\
\mathrm{DG}_{741} 282 \mathrm{kw} \text { p.f } 0.9\end{array}$ \\
\hline $75 \%$ & $\begin{array}{l}\mathrm{DG}_{724} 196 \mathrm{kw} \text { p.f } 1.0 \\
\mathrm{DG}_{718} 195 \mathrm{kw} \text { p.f } 1.0 \\
\mathrm{DG}_{742} 195 \mathrm{kw} \text { p.f } 0.9\end{array}$ & $\begin{array}{l}\mathrm{DG}_{729} 207.0 \mathrm{kw} \text { p.f } 1.0 \\
\mathrm{DG}_{731} 206.5 \mathrm{kw} \text { p.f } 0.9\end{array}$ & $\begin{array}{l}\mathrm{DG}_{736} 210 \mathrm{kw} \text { p.f } 1.0 \\
\mathrm{DG}_{741} 211 \mathrm{kw} \text { p.f } 0.9\end{array}$ \\
\hline $50 \%$ & $\begin{array}{l}\mathrm{DG}_{724} 130 \mathrm{kw} \text { p.f } 1.0 \\
\mathrm{DG}_{718} 130 \mathrm{kw} \text { p.f } 1.0 \\
\mathrm{DG}_{742} 131 \mathrm{kw} \text { p.f } 0.9\end{array}$ & $\begin{array}{l}\mathrm{DG}_{729} 137.5 \mathrm{kw} \text { p.f } 1.0 \\
\mathrm{DG}_{731} 137.0 \mathrm{kw} \text { p.f } 0.9\end{array}$ & $\begin{array}{l}\mathrm{DG}_{736} 140 \mathrm{kw} \text { p.f } 1.0 \\
\mathrm{DG}_{741} 141 \mathrm{kw} \text { p.f } 0.9\end{array}$ \\
\hline $25 \%$ & $\begin{array}{l}\mathrm{DG}_{724} 66 \mathrm{kw} \text { p.f } 1.0 \\
\mathrm{DG}_{718} 65 \mathrm{kw} \text { p.f } 1.0 \\
\mathrm{DG}_{742} 64 \mathrm{kw} \text { p.f } 0.9\end{array}$ & $\begin{array}{l}\mathrm{DG}_{729} 69.0 \mathrm{kw} \text { p.f } 1.0 \\
\mathrm{DG}_{731} 68.25 \mathrm{kw} \text { p.f } 0.9\end{array}$ & $\begin{array}{l}\mathrm{DG}_{736} 70 \mathrm{kw} \text { p.f } 1.0 \\
\mathrm{DG}_{741} 70.5 \mathrm{kw} \text { p.f } 0.9\end{array}$ \\
\hline
\end{tabular}




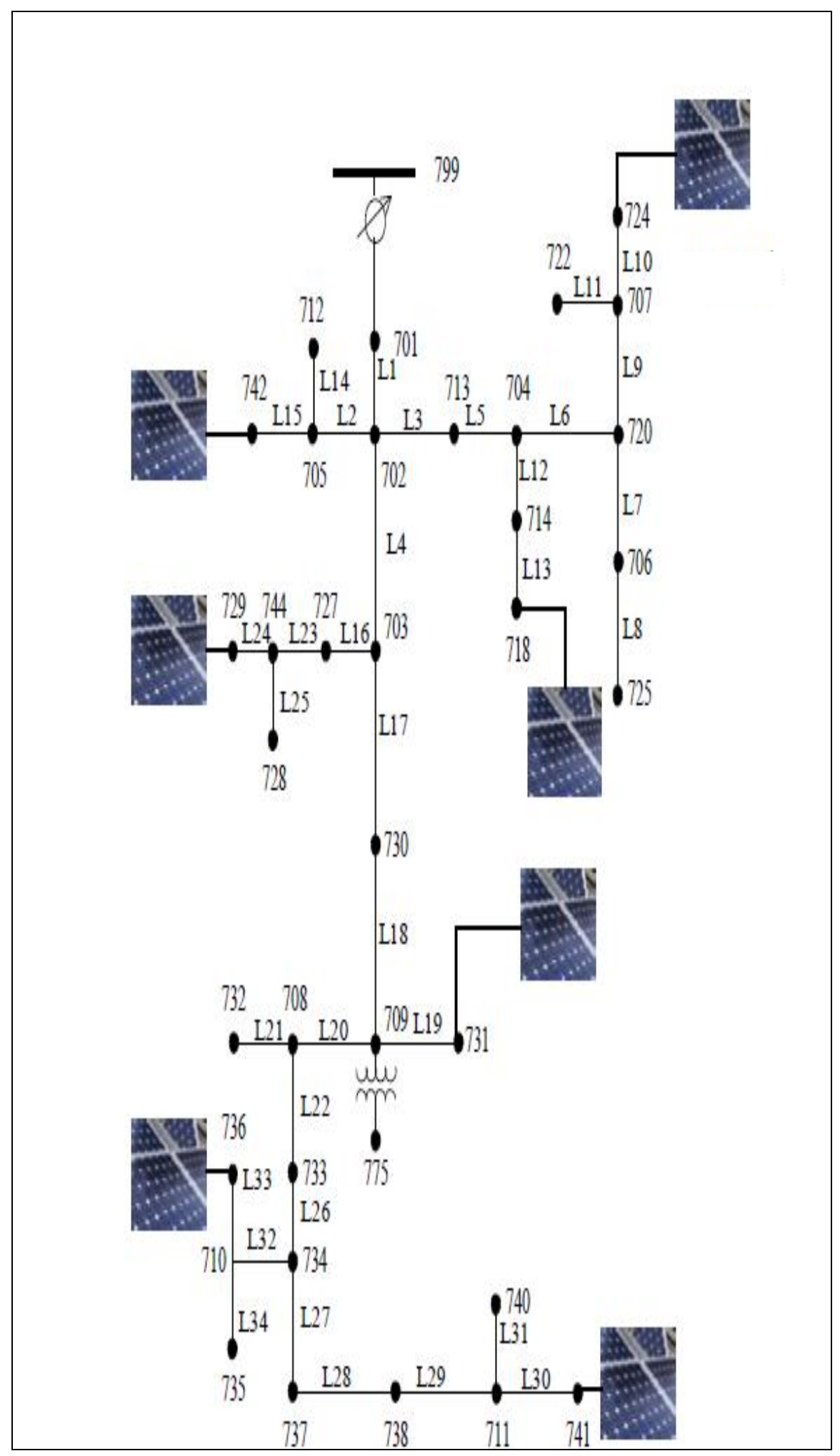

Figure 4-3: Modified IEEE test system 
The voltage, current and power flow results for the different penetration levels are presented in the following tables. The voltage magnitude and phase angle in each phase are shown at all the buses of the test system. While, the current magnitude and phase angle in each phase for each node of the distribution line are shown. For all the distribution lines, values of current are first shown at the node near the substation and then at the other node of the line. The power flow results show both the real power (kW) and reactive power (kVar) in all the lines and transformers. The power flow in the lines are shown first at the node near the substation and then at the other node of the line.

\subsubsection{0\% DG penetration case}

Table 4-2: 100\% DG penetration case voltages

\begin{tabular}{|c|c|c|c|c|c|c|c|c|c|c|c|c|c|}
\hline Bus & BasekV & Node1 & Magnitude1 & Angle1 & pu1 & Node2 & Magnitude2 & Angle2 & pu2 & Node3 & Magnitude3 & Angle3 & pu3 \\
\hline $\begin{array}{c}\text { Source } \\
\text { bus }\end{array}$ & 230 & 1 & 132791 & 0 & 1.00 & 2 & 132791 & -120 & 1.00 & 3 & 132791 & 120 & 1.00 \\
\hline 709 & 4.8 & 1 & 2672.51 & -2.6 & 0.96 & 2 & 2710.87 & -120 & 0.98 & 3 & 2797.74 & 118 & 1.01 \\
\hline 775 & 0.48 & 1 & 267.25 & -2.6 & 0.96 & 2 & 271.09 & -120 & 0.98 & 3 & 279.773 & 118 & 1.01 \\
\hline 701 & 4.8 & 1 & 2672.62 & -2.6 & 0.96 & 2 & 2712.96 & -120.1 & 0.98 & 3 & 2790.81 & 117.9 & 1.01 \\
\hline 702 & 4.8 & 1 & 2673.33 & -2.6 & 0.96 & 2 & 2713.23 & -120.1 & 0.98 & 3 & 2791.18 & 118 & 1.01 \\
\hline 705 & 4.8 & 1 & 2674.57 & -2.5 & 0.97 & 2 & 2714.44 & -120.1 & 0.98 & 3 & 2790.61 & 118 & 1.01 \\
\hline 713 & 4.8 & 1 & 2673.77 & -2.5 & 0.96 & 2 & 2713.31 & -120.2 & 0.98 & 3 & 2789.83 & 118 & 1.01 \\
\hline 703 & 4.8 & 1 & 2673.68 & -2.6 & 0.96 & 2 & 2711.8 & -120.1 & 0.98 & 3 & 2794.92 & 118 & 1.01 \\
\hline 704 & 4.8 & 1 & 2676.14 & -2.5 & 0.97 & 2 & 2713.43 & -120.2 & 0.98 & 3 & 2788.72 & 118 & 1.01 \\
\hline 720 & 4.8 & 1 & 2677.36 & -2.5 & 0.97 & 2 & 2713.88 & -120.3 & 0.98 & 3 & 2784.61 & 118 & 1.00 \\
\hline 706 & 4.8 & 1 & 2677.3 & -2.5 & 0.97 & 2 & 2713.38 & -120.3 & 0.98 & 3 & 2783.69 & 118 & 1.00 \\
\hline 725 & 4.8 & 1 & 2677.28 & -2.5 & 0.97 & 2 & 2712.85 & -120.3 & 0.98 & 3 & 2782.93 & 118 & 1.00 \\
\hline 707 & 4.8 & 1 & 2685.86 & -2.5 & 0.97 & 2 & 2714.67 & -120.5 & 0.98 & 3 & 2781.93 & 118 & 1.00 \\
\hline
\end{tabular}




\begin{tabular}{|c|c|c|c|c|c|c|c|c|c|c|c|c|c|}
\hline Bus & BasekV & Node1 & Magnitude1 & Angle1 & pu1 & Node2 & Magnitude2 & Angle2 & pu2 & Node3 & Magnitude3 & Angle3 & pu3 \\
\hline 722 & 4.8 & 1 & 2685.66 & -2.5 & 0.97 & 2 & 2713.91 & -120.5 & 0.98 & 3 & 2780.74 & 118 & 1.00 \\
\hline 724 & 4.8 & 1 & 2694.19 & -2.5 & 0.97 & 2 & 2720.15 & -120.5 & 0.98 & 3 & 2787.24 & 118.1 & 1.01 \\
\hline 714 & 4.8 & 1 & 2676.54 & -2.5 & 0.97 & 2 & 2713.45 & -120.2 & 0.98 & 3 & 2789.26 & 118 & 1.01 \\
\hline 718 & 4.8 & 1 & 2679.58 & -2.5 & 0.97 & 2 & 2714.61 & -120.1 & 0.98 & 3 & 2793.41 & 118.1 & 1.01 \\
\hline 712 & 4.8 & 1 & 2673.21 & -2.5 & 0.96 & 2 & 2714.44 & -120.1 & 0.98 & 3 & 2789.69 & 118 & 1.01 \\
\hline 742 & 4.8 & 1 & 2677.38 & -2.5 & 0.97 & 2 & 2715.41 & -120.1 & 0.98 & 3 & 2791.37 & 118 & 1.01 \\
\hline 727 & 4.8 & 1 & 2674.07 & -2.6 & 0.96 & 2 & 2711.8 & -120.1 & 0.98 & 3 & 2796.09 & 118 & 1.01 \\
\hline 730 & 4.8 & 1 & 2672.32 & -2.6 & 0.96 & 2 & 2711.1 & -120 & 0.98 & 3 & 2796.8 & 118 & 1.01 \\
\hline 731 & 4.8 & 1 & 2676.75 & -2.6 & 0.97 & 2 & 2713.19 & -120 & 0.98 & 3 & 2799.32 & 118 & 1.01 \\
\hline 708 & 4.8 & 1 & 2670.58 & -2.7 & 0.96 & 2 & 2709.27 & -120 & 0.98 & 3 & 2798.41 & 118 & 1.01 \\
\hline 732 & 4.8 & 1 & 2669.68 & -2.7 & 0.96 & 2 & 2709.27 & -120 & 0.98 & 3 & 2797.8 & 118 & 1.01 \\
\hline 733 & 4.8 & 1 & 2669.16 & -2.7 & 0.96 & 2 & 2707.66 & -119.9 & 0.98 & 3 & 2799.33 & 118 & 1.01 \\
\hline 744 & 4.8 & 1 & 2674.97 & -2.7 & 0.97 & 2 & 2711.81 & -120.1 & 0.98 & 3 & 2797.12 & 118 & 1.01 \\
\hline 729 & 4.8 & 1 & 2678.07 & -2.7 & 0.97 & 2 & 2713.88 & -120.1 & 0.98 & 3 & 2800.24 & 118 & 1.01 \\
\hline 728 & 4.8 & 1 & 2674.02 & -2.7 & 0.96 & 2 & 2710.87 & -120.1 & 0.98 & 3 & 2796.23 & 118 & 1.01 \\
\hline 734 & 4.8 & 1 & 2667.53 & -2.7 & 0.96 & 2 & 2706.57 & -119.9 & 0.98 & 3 & 2800.83 & 118 & 1.01 \\
\hline 737 & 4.8 & 1 & 2665.13 & -2.8 & 0.96 & 2 & 2703.05 & -119.8 & 0.98 & 3 & 2802.39 & 118 & 1.01 \\
\hline 738 & 4.8 & 1 & 2664.61 & -2.8 & 0.96 & 2 & 2702.88 & -119.8 & 0.98 & 3 & 2803.24 & 118 & 1.01 \\
\hline 711 & 4.8 & 1 & 2665.02 & -2.7 & 0.96 & 2 & 2704.61 & -119.8 & 0.98 & 3 & 2803.95 & 118.1 & 1.01 \\
\hline 741 & 4.8 & 1 & 2666.76 & -2.7 & 0.96 & 2 & 2706.34 & -119.7 & 0.98 & 3 & 2805.33 & 118.1 & 1.01 \\
\hline 740 & 4.8 & 1 & 2663.89 & -2.7 & 0.96 & 2 & 2704.61 & -119.8 & 0.98 & 3 & 2803.19 & 118 & 1.01 \\
\hline 710 & 4.8 & 1 & 2670.51 & -2.6 & 0.96 & 2 & 2710.2 & -119.9 & 0.98 & 3 & 2802.32 & 118.1 & 1.01 \\
\hline 736 & 4.8 & 1 & 2685.08 & -2.5 & 0.97 & 2 & 2719.12 & -119.8 & 0.98 & 3 & 2810.91 & 118.3 & 1.01 \\
\hline 735 & 4.8 & 1 & 2669.38 & -2.6 & 0.96 & 2 & 2710.2 & -119.9 & 0.98 & 3 & 2801.56 & 118 & 1.01 \\
\hline
\end{tabular}


Table 4-3: 100\% DG Penetration Case Currents

\begin{tabular}{|c|c|c|c|c|c|c|c|c|c|c|c|c|}
\hline Element & I1_1 & Ang1_1 & I1_2 & Ang1_2 & I1_3 & Ang1_3 & I2_1 & Ang2_1 & I2_2 & Ang2_2 & I2_3 & Ang2_3 \\
\hline Vsource.source & $4.18 \mathrm{E}-06$ & 90 & $4.18 \mathrm{E}-06$ & -30 & $4.18 \mathrm{E}-06$ & -150 & $4.18 \mathrm{E}-06$ & -90 & $4.18 \mathrm{E}-06$ & 150 & $4.18 \mathrm{E}-06$ & 30 \\
\hline Subxf & $4.18 \mathrm{E}-06$ & -90 & $4.18 \mathrm{E}-06$ & 150 & $4.18 \mathrm{E}-06$ & 30 & $2.33 \mathrm{E}-09$ & -7.95 & $2.71 \mathrm{E}-09$ & -120.15 & $2.42 \mathrm{E}-09$ & 122.91 \\
\hline Xfm1 & $3.87 \mathrm{E}-05$ & -92.64 & $3.92 \mathrm{E}-05$ & 149.97 & $4.05 \mathrm{E}-05$ & 27.99 & $4.07 \mathrm{E}-12$ & -153.43 & $2.03 \mathrm{E}-12$ & 153.43 & $7.50 \mathrm{E}-12$ & -14.04 \\
\hline Line.11 & 9.17 & 164.82 & 5.14 & 60.09 & 9.31 & -47.46 & 9.16 & -14.80 & 5.14 & -119.20 & 9.32 & 132.92 \\
\hline Line.12 & 18.32 & -143.38 & 9.73 & 36.20 & 8.60 & 37.13 & 18.33 & 36.65 & 9.73 & -143.73 & 8.61 & -142.89 \\
\hline Line.13 & 12.29 & -164.72 & 21.50 & -54.04 & 20.70 & 92.10 & 12.29 & 15.34 & 21.48 & 125.95 & 20.71 & -87.93 \\
\hline Line.14 & 24.21 & 42.99 & 24.15 & 138.08 & 32.71 & -89.56 & 24.15 & -137.15 & 24.24 & -41.88 & 32.66 & 90.58 \\
\hline Line.15 & 27.66 & 166.40 & 21.48 & -54.05 & 17.99 & 37.45 & 27.66 & -13.56 & 21.47 & 125.93 & 18.01 & -142.56 \\
\hline Line.16 & 13.70 & -174.22 & 34.60 & -52.28 & 29.76 & 104.65 & 13.71 & 5.91 & 34.57 & 127.70 & 29.77 & -75.41 \\
\hline Line.17 & 0.03 & 87.51 & 8.77 & -90.60 & 8.73 & 89.41 & 0.01 & -92.49 & 8.75 & 89.27 & 8.75 & -90.73 \\
\hline Line.18 & 0.01 & 87.51 & 8.75 & -90.73 & 8.75 & 89.27 & 0.00 & 100.62 & 8.75 & 89.22 & 8.75 & -90.78 \\
\hline Line.19 & 29.86 & 163.87 & 28.22 & -41.20 & 12.74 & 54.16 & 29.86 & -16.08 & 28.19 & 138.79 & 12.77 & -125.89 \\
\hline Line.110 & 4.37 & -32.75 & 29.17 & -90.84 & 31.70 & 95.89 & 4.38 & 147.21 & 29.17 & 89.15 & 31.70 & -84.12 \\
\hline Line.111 & 34.07 & 161.81 & 24.09 & 26.09 & 23.75 & -63.13 & 34.06 & -18.15 & 24.07 & -153.87 & 23.75 & 116.93 \\
\hline Line.112 & 15.41 & 149.31 & 13.16 & 130.64 & 28.19 & -39.26 & 15.41 & -30.68 & 13.16 & -49.36 & 28.19 & 140.75 \\
\hline Line.113 & 17.47 & 159.39 & 16.96 & 108.64 & 31.11 & -45.56 & 17.47 & -20.56 & 16.97 & -71.33 & 31.12 & 134.47 \\
\hline Line.114 & 17.92 & -32.95 & 0.01 & -30.14 & 17.93 & 147.05 & 17.92 & 147.03 & 0.00 & 0.00 & 17.92 & -32.97 \\
\hline Line.115 & 29.77 & -177.70 & 9.72 & 36.31 & 22.38 & -11.75 & 29.77 & 2.32 & 9.72 & -143.64 & 22.39 & 168.27 \\
\hline Line.116 & 16.32 & 86.49 & 1.00 & -48.74 & 15.62 & -96.10 & 16.32 & -93.51 & 0.99 & 131.12 & 15.62 & 83.92 \\
\hline Line.117 & 16.70 & 0.44 & 25.23 & 137.85 & 17.24 & -83.37 & 16.70 & -179.64 & 25.25 & -42.14 & 17.23 & 96.70 \\
\hline Line.118 & 9.88 & 78.25 & 25.25 & 137.86 & 31.48 & -57.96 & 9.87 & -101.75 & 25.26 & -42.14 & 31.48 & 122.06 \\
\hline Line.119 & 39.78 & 161.22 & 24.44 & 8.09 & 21.10 & -50.33 & 39.78 & -18.74 & 24.42 & -171.88 & 21.10 & 129.73 \\
\hline Line.120 & 39.80 & -4.53 & 45.00 & 162.53 & 10.93 & -72.76 & 39.80 & 175.46 & 45.02 & -17.47 & 10.93 & 107.30 \\
\hline Line.121 & 8.84 & -33.05 & 0.01 & -29.98 & 8.85 & 146.95 & 8.84 & 146.89 & 0.00 & 101.77 & 8.84 & -33.11 \\
\hline
\end{tabular}




\begin{tabular}{|c|c|c|c|c|c|c|c|c|c|c|c|c|}
\hline Element & I1_1 & Ang1_1 & I1_2 & Ang1_2 & I1_3 & Ang1_3 & I2_1 & Ang2_1 & I2_2 & Ang2_2 & I2_3 & Ang2_3 \\
\hline Line. 122 & 32.31 & 2.95 & 45.03 & 162.52 & 18.61 & -55.04 & 32.31 & -177.07 & 45.04 & -17.48 & 18.62 & 124.99 \\
\hline Line. 123 & 22.06 & 106.88 & 0.994392 & -48.88 & 21.1503 & -74.22 & 22.0494 & -73.11 & 0.984208 & 130.92 & 21.148 & 105.81 \\
\hline Line. 124 & 34.36 & 150.14 & 23.6871 & 45.14 & 36.3284 & -68.89 & 34.353 & -29.85 & 23.6849 & -134.84 & 36.3273 & 111.13 \\
\hline Line. 125 & 15.40 & -1.53 & 15.4139 & -121.59 & 15.3952 & 118.4 & 15.4036 & 178.44 & 15.4141 & 58.38 & 15.3951 & -61.62 \\
\hline Line.126 & 18.14 & -22.4 & 35.2454 & 141.18 & 18.6159 & -55.01 & 18.1469 & 157.53 & 35.2667 & -38.82 & 18.6186 & 125.06 \\
\hline Line. 127 & 34.65 & 17.6 & 38.6422 & 177.73 & 13.2764 & -65.11 & 34.6368 & -162.44 & 38.6639 & -2.28 & 13.2751 & 115 \\
\hline Line. 128 & 8.35 & -26.08 & 20.4269 & 129.94 & 13.2751 & -65 & 8.35923 & 153.83 & 20.4413 & -50.05 & 13.2742 & 115.07 \\
\hline Line.129 & 23.76 & -134.25 & 31.0042 & 69.31 & 13.2742 & -64.93 & 23.7727 & 45.78 & 31.0066 & -110.66 & 13.2734 & 115.14 \\
\hline Line. 130 & 32.37 & -167.05 & 31.0076 & 69.35 & 30.0012 & -46.6 & 32.3772 & 12.98 & 31.01 & -110.62 & 30.0054 & 133.43 \\
\hline Line.131 & 17.88 & -33.16 & 0.006172 & -29.77 & 17.8865 & 146.85 & 17.8834 & 146.83 & $2.03 \mathrm{E}-12$ & 26.57 & 17.8834 & -33.17 \\
\hline Line. 132 & 26.79 & -152 & 23.3966 & 61.58 & 14.8607 & -32.69 & 26.7993 & 28.03 & 23.397 & -118.38 & 14.8688 & 147.37 \\
\hline Line.133 & 38.74 & -175.79 & 23.3972 & 61.64 & 32.7507 & -32.86 & 38.7464 & 4.27 & 23.3982 & -118.27 & 32.7706 & 147.2 \\
\hline Line.134 & 17.88 & -33.06 & 0.006185 & -29.85 & 17.8821 & 146.94 & 17.879 & 146.92 & $2.45 \mathrm{E}-12$ & 21.8 & 17.879 & -33.08 \\
\hline
\end{tabular}


Table 4-4: 100\% DG Penetration Power Flow

\begin{tabular}{|c|c|c|c|c|c|c|c|c|c|c|c|c|}
\hline Element & kW1_1 & kvar1_1 & kW2_1 & kvar2_1 & kW3_1 & kvar3_1 & kW1_2 & kvar1 & kW2_2 & kvar2_2 & kW3_2 & kvar3_2 \\
\hline Subxf & 0.00 & 0.00 & 0.00 & 0.00 & 0.00 & 0.00 & 0.00 & 0.00 & 0.00 & 0.00 & 0.00 & 0.00 \\
\hline Xfm1 & 0.00 & 0.00 & 0.00 & 0.00 & 0.00 & 0.00 & 0.00 & 0.00 & 0.00 & 0.00 & 0.00 & 0.00 \\
\hline Line.11 & -23.93 & -5.36 & -13.94 & 0.06 & -25.13 & 6.55 & 23.93 & 5.20 & 13.94 & -0.23 & 25.14 & -6.72 \\
\hline Line.12 & -37.98 & 30.94 & -24.19 & -10.60 & 3.83 & 23.69 & 38.02 & -30.96 & 24.20 & 10.57 & -3.82 & -23.72 \\
\hline Line.13 & -31.28 & 10.06 & 23.63 & -53.33 & 52.00 & 25.20 & 31.28 & -10.09 & -23.60 & 53.30 & -51.98 & -25.23 \\
\hline Line.14 & 45.32 & -46.19 & -13.38 & 64.15 & -80.96 & -42.17 & -45.27 & 46.02 & 13.43 & -64.34 & 81.05 & 42.01 \\
\hline Line. 15 & -72.59 & -14.19 & 23.60 & -53.30 & 8.28 & 49.51 & 72.65 & 14.18 & -23.56 & 53.27 & -8.25 & -49.55 \\
\hline Line.16 & -36.29 & 5.29 & 35.28 & -87.00 & 80.75 & 19.13 & 36.30 & -5.36 & -35.13 & 86.99 & -80.64 & -19.16 \\
\hline Line. 17 & 0.00 & -0.08 & 20.66 & -11.79 & 21.37 & 11.63 & 0.00 & 0.02 & -20.66 & 11.73 & -21.36 & -11.69 \\
\hline Line.18 & 0.00 & -0.02 & 20.66 & -11.73 & 21.36 & 11.69 & 0.00 & 0.00 & -20.65 & 11.71 & -21.35 & -11.71 \\
\hline Line.19 & -77.70 & -18.86 & 14.47 & -75.20 & 15.67 & 31.84 & 77.94 & 18.86 & -14.25 & 75.19 & -15.62 & -31.90 \\
\hline Line.110 & 10.14 & 5.92 & 68.82 & -39.16 & 81.68 & 33.23 & -10.14 & -5.93 & -68.79 & 39.16 & -81.65 & -33.23 \\
\hline Line.111 & -88.08 & -24.78 & -54.57 & -36.03 & -66.07 & -1.33 & 88.35 & 24.81 & 54.70 & 36.00 & 66.19 & 1.31 \\
\hline Line.112 & -36.36 & -19.47 & -11.72 & 33.73 & -72.50 & 30.41 & 36.37 & 19.47 & 11.72 & -33.74 & 72.52 & -30.42 \\
\hline Line.113 & -44.46 & -14.53 & -30.29 & 34.65 & -83.23 & 24.56 & 44.51 & 14.50 & 30.34 & -34.68 & 83.38 & -24.56 \\
\hline Line.114 & 41.32 & 24.29 & 0.00 & -0.02 & 43.73 & -24.31 & -41.30 & -24.30 & 0.00 & 0.00 & -43.70 & 24.30 \\
\hline Line. 115 & -79.34 & 6.67 & -24.20 & -10.55 & -39.91 & 48.04 & 79.42 & -6.67 & 24.21 & 10.52 & 39.96 & -48.05 \\
\hline Line.116 & 0.68 & -43.64 & 0.87 & -2.57 & -36.17 & -24.47 & -0.66 & 43.63 & -0.87 & 2.55 & 36.19 & 24.45 \\
\hline Line.117 & 44.59 & -2.38 & -14.29 & 66.91 & -44.89 & -17.54 & -44.57 & 2.33 & 14.35 & -66.95 & 44.92 & 17.49 \\
\hline Line.118 & 4.18 & -26.06 & -14.35 & 66.95 & -87.82 & 6.24 & -4.18 & 26.05 & 14.37 & -66.96 & 87.85 & -6.24 \\
\hline Line.119 & -102.13 & -29.54 & -40.89 & -52.13 & -57.81 & 11.95 & 102.28 & 29.57 & 40.94 & 52.09 & 57.85 & -11.99 \\
\hline Line.120 & 106.31 & 3.50 & 26.52 & 119.08 & -30.04 & -5.71 & -106.23 & -3.48 & -26.42 & -119.06 & 30.05 & 5.67 \\
\hline Line.121 & 20.36 & 11.94 & 0.00 & -0.03 & 21.66 & -11.99 & -20.35 & -11.96 & 0.00 & 0.00 & -21.65 & 11.96 \\
\hline Line. 122 & 85.88 & -8.45 & 26.42 & 119.09 & -51.71 & 6.31 & -85.83 & 8.45 & -26.32 & -119.07 & 51.73 & -6.34 \\
\hline
\end{tabular}




\begin{tabular}{|c|c|c|c|c|c|c|c|c|c|c|c|c|}
\hline Element & kW1_1 & kvar1_1 & kW2_1 & kvar2_1 & kW3_1 & kvar3_1 & kW1_2 & kvar1 & kW2_2 & kvar2_2 & kW3_2 & kvar3_2 \\
\hline Line.123 & -19.72 & -55.60 & 0.87 & -2.55 & -57.81 & -12.48 & 19.74 & 55.58 & -0.87 & 2.52 & 57.83 & 12.47 \\
\hline Line.124 & -81.74 & -42.02 & -62.11 & -16.37 & -100.89 & -12.15 & 81.84 & 42.03 & 62.16 & 16.36 & 101.00 & 12.16 \\
\hline Line. 125 & 41.20 & -0.81 & 41.79 & 1.09 & 43.06 & -0.32 & -41.18 & 0.80 & -41.77 & -1.10 & -43.05 & 0.31 \\
\hline Line.126 & 45.58 & 16.34 & -14.73 & 94.29 & -51.73 & 6.34 & -45.55 & -16.38 & 14.84 & -94.29 & 51.76 & -6.39 \\
\hline Line.127 & 86.68 & -32.05 & 48.47 & 92.68 & -37.13 & -2.03 & -86.57 & 32.05 & -48.32 & -92.67 & 37.15 & 1.97 \\
\hline Line. 128 & 20.44 & 8.81 & -19.10 & 51.81 & -37.15 & -1.97 & -20.44 & -8.85 & 19.13 & -51.83 & 37.16 & 1.93 \\
\hline Line.129 & -41.94 & 47.43 & -82.75 & 13.26 & -37.16 & -1.93 & 41.98 & -47.45 & 82.81 & -13.27 & 37.17 & 1.89 \\
\hline Line.130 & -83.07 & 23.32 & -82.81 & 13.28 & -81.12 & 22.27 & 83.13 & -23.32 & 82.86 & -13.29 & 81.17 & -22.27 \\
\hline Line.131 & 41.09 & 24.14 & 0.00 & -0.02 & 43.95 & -24.16 & -41.07 & -24.15 & 0.00 & 0.00 & -43.93 & 24.15 \\
\hline Line.132 & -61.45 & 36.48 & -63.30 & 1.61 & -36.30 & 20.36 & 61.57 & -36.49 & 63.39 & -1.63 & 36.34 & -20.39 \\
\hline Line.133 & -102.73 & 12.31 & -63.39 & 1.65 & -80.22 & 44.60 & 103.32 & -12.22 & 63.60 & -1.69 & 80.62 & -44.56 \\
\hline Line.134 & 41.16 & 24.18 & 0.00 & -0.02 & 43.88 & -24.20 & -41.14 & -24.19 & 0.00 & 0.00 & -43.86 & 24.19 \\
\hline
\end{tabular}

\subsubsection{5\% DG Penetration Case}

Table 4-5: 75\% DG penetration case voltages

\begin{tabular}{|c|c|c|c|c|c|c|c|c|c|c|c|c|c|}
\hline Bus & BasekV & Node1 & Magnitude1 & Angle1 & pu1 & Node2 & Magnitude2 & Angle2 & pu2 & Node3 & Magnitude3 & Angle3 & pu3 \\
\hline $\begin{array}{c}\text { Source } \\
\text { bus }\end{array}$ & 230 & 1 & 122277 & -2.8 & 0.92 & 2 & 123123 & -121.6 & 0.93 & 3 & 124925 & 117.4 & 0.94 \\
\hline 709 & 4.8 & 1 & 2713.87 & -3.8 & 0.98 & 2 & 2748.5 & -122 & 0.99 & 3 & 2805.28 & 116.5 & 1.01 \\
\hline 775 & 0.48 & 1 & 271.384 & -3.8 & 0.98 & 2 & 274.851 & -122 & 0.99 & 3 & 280.529 & 116.5 & 1.01 \\
\hline 701 & 4.8 & 1 & 2730.21 & -3.7 & 0.99 & 2 & 2762.25 & -122.1 & 1.00 & 3 & 2811.11 & 116.6 & 1.01 \\
\hline 702 & 4.8 & 1 & 2724.42 & -3.6 & 0.98 & 2 & 2757.74 & -122.1 & 1.00 & 3 & 2806.16 & 116.6 & 1.01 \\
\hline 705 & 4.8 & 1 & 2724.8 & -3.6 & 0.98 & 2 & 2758.33 & -122.1 & 1.00 & 3 & 2804.9 & 116.6 & 1.01 \\
\hline 713 & 4.8 & 1 & 2723.43 & -3.6 & 0.98 & 2 & 2756.73 & -122.1 & 0.99 & 3 & 2803.64 & 116.6 & 1.01 \\
\hline 703 & 4.8 & 1 & 2719.28 & -3.7 & 0.98 & 2 & 2752.39 & -122 & 0.99 & 3 & 2805.5 & 116.6 & 1.01 \\
\hline 704 & 4.8 & 1 & 2723.72 & -3.6 & 0.98 & 2 & 2755.28 & -122.1 & 0.99 & 3 & 2800.82 & 116.6 & 1.01 \\
\hline
\end{tabular}




\begin{tabular}{|c|c|c|c|c|c|c|c|c|c|c|c|c|c|}
\hline Bus & BasekV & Node1 & Magnitude1 & Angle1 & pu1 & Node2 & Magnitude2 & Angle2 & pu2 & Node3 & Magnitude3 & Angle3 & pu3 \\
\hline 720 & 4.8 & 1 & 2722.79 & -3.5 & 0.98 & 2 & 2754.05 & -122.2 & 0.99 & 3 & 2794.81 & 116.6 & 1.01 \\
\hline 706 & 4.8 & 1 & 2722.73 & -3.5 & 0.98 & 2 & 2753.56 & -122.2 & 0.99 & 3 & 2793.89 & 116.6 & 1.01 \\
\hline 725 & 4.8 & 1 & 2722.71 & -3.5 & 0.98 & 2 & 2753.03 & -122.2 & 0.99 & 3 & 2793.15 & 116.6 & 1.01 \\
\hline 707 & 4.8 & 1 & 2727.58 & -3.4 & 0.98 & 2 & 2752.05 & -122.3 & 0.99 & 3 & 2788.87 & 116.7 & 1.01 \\
\hline 722 & 4.8 & 1 & 2727.38 & -3.4 & 0.98 & 2 & 2751.29 & -122.3 & 0.99 & 3 & 2787.68 & 116.7 & 1.01 \\
\hline 724 & 4.8 & 1 & 2732.86 & -3.4 & 0.99 & 2 & 2755.22 & -122.2 & 0.99 & 3 & 2791.47 & 116.8 & 1.01 \\
\hline 714 & 4.8 & 1 & 2723.95 & -3.6 & 0.98 & 2 & 2755.17 & -122.1 & 0.99 & 3 & 2801.23 & 116.6 & 1.01 \\
\hline 718 & 4.8 & 1 & 2725.81 & -3.6 & 0.98 & 2 & 2755.51 & -122 & 0.99 & 3 & 2804.52 & 116.7 & 1.01 \\
\hline 712 & 4.8 & 1 & 2723.46 & -3.6 & 0.98 & 2 & 2758.33 & -122.1 & 1.00 & 3 & 2804 & 116.6 & 1.01 \\
\hline 742 & 4.8 & 1 & 2726.9 & -3.6 & 0.98 & 2 & 2758.81 & -122.1 & 1.00 & 3 & 2805.1 & 116.6 & 1.01 \\
\hline 727 & 4.8 & 1 & 2718.57 & -3.7 & 0.98 & 2 & 2751.59 & -122 & 0.99 & 3 & 2805.7 & 116.6 & 1.01 \\
\hline 730 & 4.8 & 1 & 2714.73 & -3.7 & 0.98 & 2 & 2749.47 & -122 & 0.99 & 3 & 2805.1 & 116.5 & 1.01 \\
\hline 731 & 4.8 & 1 & 2716.21 & -3.7 & 0.98 & 2 & 2749.37 & -121.9 & 0.99 & 3 & 2805.19 & 116.6 & 1.01 \\
\hline 708 & 4.8 & 1 & 2711.24 & -3.8 & 0.98 & 2 & 2746.48 & -121.9 & 0.99 & 3 & 2805.62 & 116.5 & 1.01 \\
\hline 732 & 4.8 & 1 & 2710.35 & -3.8 & 0.98 & 2 & 2746.48 & -121.9 & 0.99 & 3 & 2805.03 & 116.5 & 1.01 \\
\hline 733 & 4.8 & 1 & 2709.13 & -3.8 & 0.98 & 2 & 2744.46 & -121.9 & 0.99 & 3 & 2806.21 & 116.5 & 1.01 \\
\hline 744 & 4.8 & 1 & 2718.59 & -3.7 & 0.98 & 2 & 2750.94 & -122 & 0.99 & 3 & 2805.97 & 116.6 & 1.01 \\
\hline 729 & 4.8 & 1 & 2720.38 & -3.7 & 0.98 & 2 & 2752.05 & -122 & 0.99 & 3 & 2807.94 & 116.6 & 1.01 \\
\hline 728 & 4.8 & 1 & 2717.65 & -3.7 & 0.98 & 2 & 2750.02 & -122 & 0.99 & 3 & 2805.09 & 116.6 & 1.01 \\
\hline 734 & 4.8 & 1 & 2706.3 & -3.9 & 0.98 & 2 & 2742.65 & -121.9 & 0.99 & 3 & 2807.14 & 116.5 & 1.01 \\
\hline 737 & 4.8 & 1 & 2702.82 & -3.9 & 0.98 & 2 & 2738.43 & -121.8 & 0.99 & 3 & 2807.96 & 116.5 & 1.01 \\
\hline 738 & 4.8 & 1 & 2701.65 & -3.9 & 0.97 & 2 & 2737.81 & -121.8 & 0.99 & 3 & 2808.34 & 116.5 & 1.01 \\
\hline 711 & 4.8 & 1 & 2701.39 & -3.9 & 0.97 & 2 & 2739.07 & -121.7 & 0.99 & 3 & 2808.6 & 116.5 & 1.01 \\
\hline 741 & 4.8 & 1 & 2702.46 & -3.9 & 0.98 & 2 & 2740.33 & -121.7 & 0.99 & 3 & 2809.5 & 116.5 & 1.01 \\
\hline 740 & 4.8 & 1 & 2700.27 & -3.9 & 0.97 & 2 & 2739.07 & -121.7 & 0.99 & 3 & 2807.84 & 116.5 & 1.01 \\
\hline 710 & 4.8 & 1 & 2708.44 & -3.8 & 0.98 & 2 & 2745.74 & -121.9 & 0.99 & 3 & 2808.19 & 116.5 & 1.01 \\
\hline
\end{tabular}




\begin{tabular}{|c|c|c|c|c|c|c|c|c|c|c|c|c|c|}
\hline Bus & BasekV & Node1 & Magnitude1 & Angle1 & pu1 & Node2 & Magnitude2 & Angle2 & pu2 & Node3 & Magnitude3 & Angle3 & pu3 \\
\hline 736 & 4.8 & 1 & 2720.89 & -3.8 & 0.98 & 2 & 2753.33 & -121.9 & 0.99 & 3 & 2815.6 & 116.6 & 1.02 \\
\hline 735 & 4.8 & 1 & 2707.32 & -3.8 & 0.98 & 2 & 2745.74 & -121.9 & 0.99 & 3 & 2807.43 & 116.5 & 1.01 \\
\hline
\end{tabular}

Table 4-6: 75\% DG Penetration Case Currents

\begin{tabular}{|c|c|c|c|c|c|c|c|c|c|c|c|c|}
\hline Element & I1_1 & Ang1_1 & I1_2 & Ang1_2 & I1_3 & Ang1_3 & I2_1 & Ang2_1 & I2_2 & Ang2_2 & I2_3 & Ang2_3 \\
\hline Vsource & 1.74 & 133.67 & 1.47 & 3.99 & 1.38 & -100.87 & 1.74 & -46.33 & 1.47 & -176.01 & 1.38 & 79.13 \\
\hline Subxf & 1.74 & -46.33 & 1.47 & -176.01 & 1.38 & 79.13 & 83.35 & 133.67 & 70.24 & 3.99 & 66.36 & -100.87 \\
\hline $\mathrm{Xfm} 1$ & 0.00 & -93.75 & 0.00 & 148.05 & 0.00 & 26.54 & 0.00 & 180.00 & 0.00 & -26.57 & 0.00 & 180.00 \\
\hline Line.11 & 68.07 & -50.13 & 61.07 & -179.89 & 55.18 & 71.59 & 68.12 & 129.84 & 61.12 & 0.08 & 55.23 & -108.46 \\
\hline Line.12 & 16.45 & -123.17 & 4.79 & 46.23 & 11.79 & 61.16 & 16.46 & 56.85 & 4.78 & -133.62 & 11.80 & -118.88 \\
\hline Line.13 & 22.47 & -86.98 & 18.27 & -120.80 & 39.03 & 77.90 & 22.48 & 93.02 & 18.27 & 59.15 & 39.04 & -102.12 \\
\hline Line.14 & 53.92 & -17.38 & 56.40 & 167.56 & 5.32 & 47.14 & 53.94 & 162.53 & 56.49 & -12.47 & 5.40 & -133.19 \\
\hline Line. 15 & 18.43 & -137.19 & 18.27 & -120.85 & 36.36 & 50.97 & 18.45 & 42.85 & 18.27 & 59.09 & 36.38 & -129.05 \\
\hline Line.16 & 18.69 & -110.97 & 24.20 & -80.98 & 41.48 & 85.99 & 18.72 & 69.06 & 24.18 & 98.97 & 41.49 & -94.05 \\
\hline Line.17 & 0.03 & 86.48 & 8.71 & -92.38 & 8.68 & 87.63 & 0.01 & -93.52 & 8.70 & 87.49 & 8.69 & -92.51 \\
\hline Line.18 & 0.01 & 86.48 & 8.70 & -92.51 & 8.69 & 87.49 & 0.00 & 161.57 & 8.69 & 87.44 & 8.69 & -92.56 \\
\hline Line. 19 & 22.76 & -160.48 & 15.73 & -74.78 & 28.64 & 52.78 & 22.77 & 19.59 & 15.71 & 105.15 & 28.67 & -127.25 \\
\hline Line.110 & 4.37 & -33.67 & 29.17 & -92.55 & 31.65 & 94.24 & 4.38 & 146.29 & 29.17 & 87.44 & 31.65 & -85.76 \\
\hline Line.111 & 25.63 & -168.27 & 14.98 & 68.87 & 21.55 & -23.93 & 25.63 & 11.78 & 14.99 & -111.04 & 21.56 & 156.12 \\
\hline Line.112 & 8.42 & 144.32 & 15.53 & 148.03 & 23.93 & -33.25 & 8.41 & -35.66 & 15.54 & -31.97 & 23.94 & 146.76 \\
\hline Line.113 & 10.51 & 161.82 & 17.30 & 124.69 & 26.44 & -41.41 & 10.50 & -18.10 & 17.31 & -55.29 & 26.45 & 138.62 \\
\hline Line.114 & 17.74 & -33.98 & 0.01 & -32.08 & 17.75 & 146.02 & 17.74 & 146.00 & 0.00 & 132.51 & 17.74 & -34.00 \\
\hline Line.115 & 24.03 & -170.73 & 4.78 & 46.47 & 20.42 & 1.17 & 24.03 & 9.29 & 4.78 & -133.41 & 20.43 & -178.82 \\
\hline Line.116 & 9.60 & -16.75 & 18.26 & 167.34 & 8.71 & -8.14 & 9.60 & 163.21 & 18.27 & -12.67 & 8.72 & 171.89 \\
\hline
\end{tabular}




\begin{tabular}{|c|c|c|c|c|c|c|c|c|c|c|c|c|}
\hline Element & I1_1 & Ang1_1 & I1_2 & Ang1_2 & I1_3 & Ang1_3 & I2_1 & Ang2_1 & I2_2 & Ang2_2 & I2_3 & Ang2_3 \\
\hline Line. 117 & 44.34 & -17.62 & 38.22 & 167.61 & 7.14 & 133.61 & 44.35 & 162.35 & 38.25 & -12.40 & 7.14 & -46.57 \\
\hline Line.118 & 27.87 & -7.29 & 38.25 & 167.60 & 10.80 & -25.97 & 27.87 & 172.70 & 38.25 & -12.40 & 10.81 & 154.06 \\
\hline Line.119 & 29.66 & -169.87 & 9.05 & 53.07 & 23.85 & -4.85 & 29.67 & 10.17 & 9.04 & -126.78 & 23.87 & 175.18 \\
\hline Line. 120 & 56.87 & 1.69 & 42.81 & 178.68 & 14.30 & -169.09 & 56.86 & -178.33 & 42.82 & -1.33 & 14.29 & 10.90 \\
\hline Line.121 & 8.77 & -34.15 & 0.01 & -31.93 & 8.78 & 145.85 & 8.78 & 145.79 & 0.00 & -144.78 & 8.78 & -34.21 \\
\hline Line.122 & 50.01 & 7.57 & 42.83 & 178.67 & 10.20 & -131.55 & 50.01 & -172.45 & 42.84 & -1.34 & 10.19 & 48.48 \\
\hline Line.123 & 2.88 & 48.00 & 18.27 & 167.33 & 17.04 & -21.14 & 2.87 & -132.13 & 18.28 & -12.68 & 17.05 & 158.89 \\
\hline Line.124 & 21.26 & -176.82 & 20.96 & 94.33 & 30.15 & -40.84 & 21.26 & 3.20 & 20.96 & -85.65 & 30.15 & 139.18 \\
\hline Line. 125 & 15.23 & -3.01 & 15.24 & -123.03 & 15.23 & 116.96 & 15.23 & 176.97 & 15.24 & 56.94 & 15.23 & -63.06 \\
\hline Line.126 & 33.89 & -2.64 & 28.60 & 161.36 & 10.19 & -131.52 & 33.89 & 177.32 & 28.62 & -18.65 & 10.17 & 48.53 \\
\hline Line.127 & 41.87 & 8.06 & 43.84 & -179.21 & 5.81 & -65.76 & 41.86 & -171.97 & 43.86 & 0.78 & 5.81 & 114.49 \\
\hline Line.128 & 17.29 & -25.92 & 22.05 & 144.49 & 5.81 & -65.51 & 17.29 & 154.03 & 22.07 & -35.51 & 5.81 & 114.65 \\
\hline Line.129 & 21.73 & -112.95 & 25.97 & 76.51 & 5.81 & -65.35 & 21.74 & 67.06 & 25.97 & -103.45 & 5.81 & 114.80 \\
\hline Line.130 & 25.22 & -156.65 & 25.98 & 76.56 & 22.97 & -41.79 & 25.23 & 23.38 & 25.98 & -103.41 & 22.97 & 138.25 \\
\hline Line.131 & 17.78 & -34.33 & 0.01 & -31.75 & 17.79 & 145.67 & 17.78 & 145.65 & 0.00 & -33.69 & 17.78 & -34.35 \\
\hline Line.132 & 15.07 & -170.42 & 19.36 & 30.27 & 7.50 & -104.40 & 15.07 & 9.64 & 19.35 & -149.69 & 7.49 & 75.69 \\
\hline Line.133 & 30.48 & 165.79 & 19.35 & 30.33 & 21.51 & -53.36 & 30.47 & -14.13 & 19.33 & -149.56 & 21.52 & 126.74 \\
\hline Line.134 & 17.77 & -34.27 & 0.01 & -31.87 & 17.77 & 145.74 & 17.77 & 145.72 & 0.00 & -67.38 & 17.77 & -34.28 \\
\hline Line.135 & 75.77 & -46.33 & 63.85 & -176.01 & 60.33 & 79.13 & 75.88 & 133.59 & 63.98 & 3.90 & 60.42 & -100.99 \\
\hline
\end{tabular}


Table 4-7: 75\% DG Penetration Power Flow

\begin{tabular}{|c|c|c|c|c|c|c|c|c|c|c|c|c|}
\hline Element & kW1_1 & kvar1_1 & kW2_1 & kvar2_1 & kW3_1 & kvar3_1 & kW1_2 & kvar1 & kW2_2 & kvar2_2 & kW3_2 & kvar3_2 \\
\hline Subxf & 154.33 & 146.37 & 105.13 & 146.70 & 135.79 & 107.21 & 395.24 & 0.00 & -158.07 & -121.11 & -114.57 & -157.15 \\
\hline Xfm1 & 0.00 & 0.00 & 0.00 & 0.00 & 0.00 & 0.00 & 0.00 & 0.00 & 0.00 & 0.00 & 0.00 & 0.00 \\
\hline Line.11 & 128.00 & 134.75 & 89.90 & 142.73 & 109.73 & 109.66 & -127.71 & -134.66 & -89.71 & -142.71 & -109.56 & -109.62 \\
\hline Line.12 & -22.09 & 39.00 & -12.92 & -2.67 & 18.79 & 27.24 & 22.12 & -39.02 & 12.93 & 2.64 & -18.78 & -27.27 \\
\hline Line.13 & 7.11 & 60.79 & 50.37 & -1.12 & 85.52 & 68.41 & -7.08 & -60.81 & -50.35 & 1.09 & -85.44 & -68.40 \\
\hline Line.14 & 142.69 & 34.86 & 52.26 & 146.50 & 5.25 & 13.97 & -142.46 & -34.86 & -52.04 & -146.51 & -5.25 & -14.22 \\
\hline Line.15 & -34.60 & 36.38 & 50.35 & -1.09 & 42.11 & 92.84 & 34.63 & -36.41 & -50.32 & 1.05 & -42.01 & -92.83 \\
\hline Line.16 & -15.21 & 48.59 & 50.22 & -43.85 & 99.97 & 59.17 & 15.26 & -48.64 & -50.15 & 43.80 & -99.76 & -59.13 \\
\hline Line.17 & 0.00 & -0.09 & 20.81 & -11.92 & 21.21 & 11.75 & 0.00 & 0.02 & -20.81 & 11.86 & -21.21 & -11.81 \\
\hline Line.18 & 0.00 & -0.02 & 20.81 & -11.86 & 21.21 & 11.81 & 0.00 & 0.00 & -20.80 & 11.83 & -21.20 & -11.83 \\
\hline Line.19 & -57.02 & 24.26 & 29.33 & -31.88 & 35.31 & 71.85 & 57.16 & -24.29 & -29.26 & 31.82 & -35.07 & -71.85 \\
\hline Line.110 & 10.31 & 6.01 & 69.72 & -39.78 & 81.56 & 33.76 & -10.30 & -6.02 & -69.69 & 39.78 & -81.52 & -33.76 \\
\hline Line.111 & -67.46 & 18.28 & -40.46 & 7.96 & -46.48 & 38.09 & 67.62 & -18.29 & 40.51 & -8.01 & 46.59 & -38.12 \\
\hline Line.112 & -19.42 & -12.18 & 0.10 & 42.80 & -57.97 & 33.67 & 19.42 & 12.17 & -0.10 & -42.81 & 57.98 & -33.67 \\
\hline Line.113 & -27.70 & -7.21 & -18.79 & 43.80 & -68.69 & 27.71 & 27.72 & 7.18 & 18.83 & -43.84 & 68.80 & -27.72 \\
\hline Line.114 & 41.70 & 24.44 & 0.00 & -0.02 & 43.35 & -24.47 & -41.68 & -24.45 & 0.00 & 0.00 & -43.32 & 24.45 \\
\hline Line.115 & -63.82 & 14.58 & -12.93 & -2.62 & -24.57 & 51.74 & 63.88 & -14.59 & 12.93 & 2.59 & 24.61 & -51.75 \\
\hline Line.116 & 25.42 & 5.88 & 16.67 & 47.42 & -13.91 & 20.10 & -25.41 & -5.90 & -16.65 & -47.44 & 13.92 & -20.12 \\
\hline Line.117 & 117.04 & 28.98 & 35.36 & 99.08 & 19.16 & -5.88 & -116.86 & -28.94 & -35.23 & -99.08 & -19.16 & 5.82 \\
\hline Line.118 & 75.51 & 4.68 & 35.23 & 99.08 & -24.04 & 18.44 & -75.49 & -4.68 & -35.18 & -99.08 & 24.04 & -18.46 \\
\hline Line.119 & -78.15 & 19.31 & -24.77 & -2.16 & -34.85 & 57.11 & 78.23 & -19.32 & 24.78 & 2.10 & 34.90 & -57.14 \\
\hline Line. 120 & 153.63 & -14.63 & 59.95 & 101.23 & 10.81 & -38.64 & -153.47 & 14.69 & -59.86 & -101.22 & -10.80 & 38.62 \\
\hline Line.121 & 20.53 & 12.02 & 0.00 & -0.03 & 21.49 & -12.07 & -20.52 & -12.05 & 0.00 & 0.00 & -21.48 & 12.05 \\
\hline Line.122 & 132.94 & -26.71 & 59.86 & 101.25 & -10.69 & -26.54 & -132.82 & 26.75 & -59.77 & -101.23 & 10.70 & 26.51 \\
\hline
\end{tabular}




\begin{tabular}{|c|c|c|c|c|c|c|c|c|c|c|c|c|}
\hline Element & kW1_1 & kvar1_1 & kW2_1 & kvar2_1 & kW3_1 & kvar3_1 & kW1_2 & kvar1 & kW2_2 & kvar2_2 & kW3_2 & kvar3_2 \\
\hline Line.123 & 4.85 & -6.15 & 16.65 & 47.44 & -35.36 & 32.17 & -4.85 & 6.12 & -16.63 & -47.46 & 35.37 & -32.20 \\
\hline Line. 124 & -57.38 & 6.94 & -46.45 & 34.15 & -78.10 & 32.48 & 57.42 & -6.96 & 46.48 & -34.16 & 78.18 & -32.48 \\
\hline Line. 125 & 41.40 & -0.52 & 41.91 & 0.76 & 42.73 & -0.29 & -41.39 & 0.50 & -41.90 & -0.77 & -42.72 & 0.27 \\
\hline Line.126 & 91.78 & -1.91 & 18.00 & 76.39 & -10.70 & -26.51 & -91.69 & 1.91 & -17.93 & -76.41 & 10.71 & 26.45 \\
\hline Line. 127 & 110.86 & -23.42 & 64.86 & 101.24 & -16.30 & -0.64 & -110.69 & 23.45 & -64.67 & -101.21 & 16.31 & 0.57 \\
\hline Line.128 & 43.32 & 17.49 & -3.90 & 60.26 & -16.31 & -0.57 & -43.31 & -17.52 & 3.93 & -60.29 & 16.31 & 0.53 \\
\hline Line.129 & -19.12 & 55.50 & -67.51 & 22.31 & -16.31 & -0.53 & 19.15 & -55.52 & 67.55 & -22.33 & 16.31 & 0.48 \\
\hline Line. 130 & -60.57 & 31.20 & -67.55 & 22.35 & -59.93 & 23.86 & 60.61 & -31.22 & 67.59 & -22.37 & 59.96 & -23.89 \\
\hline Line.131 & 41.42 & 24.33 & 0.00 & -0.02 & 43.62 & -24.35 & -41.40 & -24.34 & 0.00 & 0.00 & -43.60 & 24.34 \\
\hline Line. 132 & -39.66 & 9.48 & -46.93 & -24.82 & -15.91 & -13.78 & 39.70 & -9.52 & 46.99 & 24.79 & 15.92 & 13.74 \\
\hline Line.133 & -81.19 & -14.85 & -46.99 & -24.78 & -59.47 & 10.65 & 81.56 & 14.86 & 47.14 & 24.71 & 59.64 & -10.70 \\
\hline Line.134 & 41.49 & 24.37 & 0.00 & -0.02 & 43.55 & -24.39 & -41.48 & -24.38 & 0.00 & 0.00 & -43.53 & 24.38 \\
\hline
\end{tabular}

\subsubsection{0\% DG penetration case}

Table 4-8: 50\% DG penetration case voltages

\begin{tabular}{|c|c|c|c|c|c|c|c|c|c|c|c|c|c|}
\hline Bus & BasekV & Node1 & Magnitude1 & Angle1 & pu1 & Node2 & Magnitude2 & Angle2 & pu2 & Node3 & Magnitude3 & Angle3 & pu3 \\
\hline $\begin{array}{c}\text { Source } \\
\text { bus }\end{array}$ & 230.00 & 1.00 & 123771.00 & -4.50 & 0.93 & 2.00 & 124689.00 & -123.40 & 0.94 & 3.00 & 126299.00 & 115.70 & 0.95 \\
\hline $\begin{array}{c}\text { Source } \\
\text { bus }\end{array}$ & 4.80 & 1.00 & 2715.90 & -6.70 & 0.98 & 2.00 & 2761.89 & -124.80 & 1.00 & 3.00 & 2819.02 & 113.40 & 1.02 \\
\hline 709 & 0.48 & 1.00 & 271.59 & -6.70 & 0.98 & 2.00 & 276.19 & -124.80 & 1.00 & 3.00 & 281.90 & 113.40 & 1.02 \\
\hline 775 & 4.80 & 1.00 & 2743.36 & -6.50 & 0.99 & 2.00 & 2783.94 & -124.80 & 1.00 & 3.00 & 2833.23 & 113.70 & 1.02 \\
\hline 701 & 4.80 & 1.00 & 2734.07 & -6.60 & 0.99 & 2.00 & 2776.95 & -124.90 & 1.00 & 3.00 & 2825.80 & 113.60 & 1.02 \\
\hline 702 & 4.80 & 1.00 & 2733.73 & -6.50 & 0.99 & 2.00 & 2776.89 & -124.90 & 1.00 & 3.00 & 2823.91 & 113.60 & 1.02 \\
\hline 705 & 4.80 & 1.00 & 2732.45 & -6.50 & 0.99 & 2.00 & 2775.38 & -124.90 & 1.00 & 3.00 & 2822.80 & 113.50 & 1.02 \\
\hline
\end{tabular}




\begin{tabular}{|c|c|c|c|c|c|c|c|c|c|c|c|c|c|}
\hline Bus & BasekV & Node1 & Magnitude1 & Angle1 & pu1 & Node2 & Magnitude2 & Angle2 & pu2 & Node3 & Magnitude3 & Angle3 & pu3 \\
\hline 713 & 4.80 & 1.00 & 2725.39 & -6.70 & 0.98 & 2.00 & 2769.09 & -124.90 & 1.00 & 3.00 & 2822.52 & 113.50 & 1.02 \\
\hline 703 & 4.80 & 1.00 & 2731.82 & -6.50 & 0.99 & 2.00 & 2773.12 & -124.90 & 1.00 & 3.00 & 2819.29 & 113.60 & 1.02 \\
\hline 704 & 4.80 & 1.00 & 2730.21 & -6.50 & 0.99 & 2.00 & 2771.27 & -125.00 & 1.00 & 3.00 & 2812.76 & 113.60 & 1.02 \\
\hline 720 & 4.80 & 1.00 & 2730.16 & -6.50 & 0.99 & 2.00 & 2770.77 & -125.00 & 1.00 & 3.00 & 2811.85 & 113.60 & 1.01 \\
\hline 706 & 4.80 & 1.00 & 2730.13 & -6.50 & 0.99 & 2.00 & 2770.25 & -125.00 & 1.00 & 3.00 & 2811.11 & 113.60 & 1.01 \\
\hline 725 & 4.80 & 1.00 & 2733.39 & -6.40 & 0.99 & 2.00 & 2767.76 & -125.10 & 1.00 & 3.00 & 2805.41 & 113.70 & 1.01 \\
\hline 707 & 4.80 & 1.00 & 2733.18 & -6.40 & 0.99 & 2.00 & 2767.00 & -125.10 & 1.00 & 3.00 & 2804.22 & 113.70 & 1.01 \\
\hline 722 & 4.80 & 1.00 & 2737.32 & -6.40 & 0.99 & 2.00 & 2769.70 & -125.10 & 1.00 & 3.00 & 2806.84 & 113.70 & 1.01 \\
\hline 724 & 4.80 & 1.00 & 2731.89 & -6.50 & 0.99 & 2.00 & 2772.88 & -124.90 & 1.00 & 3.00 & 2819.57 & 113.60 & 1.02 \\
\hline 714 & 4.80 & 1.00 & 2732.78 & -6.50 & 0.99 & 2.00 & 2772.36 & -124.90 & 1.00 & 3.00 & 2822.04 & 113.60 & 1.02 \\
\hline 718 & 4.80 & 1.00 & 2732.39 & -6.50 & 0.99 & 2.00 & 2776.90 & -124.90 & 1.00 & 3.00 & 2823.02 & 113.50 & 1.02 \\
\hline 712 & 4.80 & 1.00 & 2735.25 & -6.50 & 0.99 & 2.00 & 2776.85 & -124.90 & 1.00 & 3.00 & 2823.60 & 113.60 & 1.02 \\
\hline 742 & 4.80 & 1.00 & 2724.08 & -6.70 & 0.98 & 2.00 & 2767.78 & -124.80 & 1.00 & 3.00 & 2822.22 & 113.50 & 1.02 \\
\hline 727 & 4.80 & 1.00 & 2717.78 & -6.70 & 0.98 & 2.00 & 2763.69 & -124.80 & 1.00 & 3.00 & 2819.65 & 113.40 & 1.02 \\
\hline 730 & 4.80 & 1.00 & 2717.48 & -6.70 & 0.98 & 2.00 & 2762.13 & -124.80 & 1.00 & 3.00 & 2818.36 & 113.50 & 1.02 \\
\hline 731 & 4.80 & 1.00 & 2712.05 & -6.80 & 0.98 & 2.00 & 2758.88 & -124.80 & 1.00 & 3.00 & 2818.34 & 113.40 & 1.02 \\
\hline 708 & 4.80 & 1.00 & 2711.17 & -6.80 & 0.98 & 2.00 & 2758.88 & -124.80 & 1.00 & 3.00 & 2817.75 & 113.40 & 1.02 \\
\hline 732 & 4.80 & 1.00 & 2708.73 & -6.80 & 0.98 & 2.00 & 2755.87 & -124.70 & 0.99 & 3.00 & 2817.93 & 113.40 & 1.02 \\
\hline 733 & 4.80 & 1.00 & 2723.75 & -6.70 & 0.98 & 2.00 & 2766.85 & -124.80 & 1.00 & 3.00 & 2822.23 & 113.50 & 1.02 \\
\hline 744 & 4.80 & 1.00 & 2724.82 & -6.70 & 0.98 & 2.00 & 2767.36 & -124.80 & 1.00 & 3.00 & 2823.60 & 113.50 & 1.02 \\
\hline 729 & 4.80 & 1.00 & 2722.81 & -6.70 & 0.98 & 2.00 & 2765.93 & -124.80 & 1.00 & 3.00 & 2821.35 & 113.50 & 1.02 \\
\hline 728 & 4.80 & 1.00 & 2703.76 & -6.90 & 0.98 & 2.00 & 2752.32 & -124.70 & 0.99 & 3.00 & 2817.08 & 113.40 & 1.02 \\
\hline 734 & 4.80 & 1.00 & 2699.44 & -7.00 & 0.97 & 2.00 & 2747.43 & -124.70 & 0.99 & 3.00 & 2817.29 & 113.30 & 1.02 \\
\hline 737 & 4.80 & 1.00 & 2697.72 & -7.00 & 0.97 & 2.00 & 2746.40 & -124.60 & 0.99 & 3.00 & 2817.29 & 113.30 & 1.02 \\
\hline 738 & 4.80 & 1.00 & 2696.93 & -6.90 & 0.97 & 2.00 & 2747.24 & -124.60 & 0.99 & 3.00 & 2817.17 & 113.30 & 1.02 \\
\hline 711 & 4.80 & 1.00 & 2697.46 & -6.90 & 0.97 & 2.00 & 2748.08 & -124.60 & 0.99 & 3.00 & 2817.69 & 113.30 & 1.02 \\
\hline
\end{tabular}




\begin{tabular}{|c|c|c|c|c|c|c|c|c|c|c|c|c|c|}
\hline Bus & BasekV & Node1 & Magnitude1 & Angle1 & pu1 & Node2 & Magnitude2 & Angle2 & pu2 & Node3 & Magnitude3 & Angle3 & pu3 \\
\hline 741 & 4.80 & 1.00 & 2695.81 & -6.90 & 0.97 & 2.00 & 2747.24 & -124.60 & 0.99 & 3.00 & 2816.42 & 113.30 & 1.02 \\
\hline 740 & 4.80 & 1.00 & 2703.84 & -6.80 & 0.98 & 2.00 & 2753.70 & -124.70 & 0.99 & 3.00 & 2816.26 & 113.40 & 1.02 \\
\hline 710 & 4.80 & 1.00 & 2711.20 & -6.70 & 0.98 & 2.00 & 2757.08 & -124.70 & 0.99 & 3.00 & 2819.09 & 113.50 & 1.02 \\
\hline 736 & 4.80 & 1.00 & 2702.72 & -6.80 & 0.98 & 2.00 & 2753.70 & -124.70 & 0.99 & 3.00 & 2815.51 & 113.30 & 1.02 \\
\hline
\end{tabular}

Table 4-9: 50\% DG Penetration Case Currents

\begin{tabular}{|c|c|c|c|c|c|c|c|c|c|c|c|c|}
\hline Element & I1_1 & Ang1_1 & I1_2 & Ang1_2 & I1_3 & Ang1_3 & I2_1 & Ang2_1 & I2_2 & Ang2_2 & I2_3 & Ang2_3 \\
\hline Vsource & 2.80 & 149.74 & 2.30 & 25.33 & 2.42 & -82.01 & 2.80 & -30.26 & 2.30 & -154.67 & 2.42 & 97.99 \\
\hline Subxf & 2.80 & -30.26 & 2.30 & -154.67 & 2.42 & 97.99 & 134.04 & 149.74 & 110.28 & 25.33 & 115.85 & -82.01 \\
\hline Xfm1 & 0.00 & -96.74 & 0.00 & 145.20 & 0.00 & 23.43 & 0.00 & -119.74 & 0.00 & -153.43 & 0.00 & 0.00 \\
\hline Line.11 & 113.04 & -31.12 & 95.96 & -156.19 & 97.58 & 95.28 & 113.07 & 148.85 & 95.99 & 23.77 & 97.60 & -84.76 \\
\hline Line.12 & 12.63 & -104.47 & 3.00 & -59.31 & 14.92 & 83.78 & 12.65 & 75.54 & 2.99 & 120.59 & 14.92 & -96.26 \\
\hline Line.13 & 24.01 & -56.94 & 30.34 & -117.82 & 47.00 & 88.70 & 24.02 & 123.04 & 30.34 & 62.15 & 47.00 & -91.31 \\
\hline Line.14 & 90.67 & -16.75 & 75.79 & -172.96 & 37.23 & 108.15 & 90.69 & 163.19 & 75.86 & 6.99 & 37.24 & -71.99 \\
\hline Line. 15 & 9.55 & -96.01 & 30.34 & -117.85 & 39.39 & 67.34 & 9.57 & 83.99 & 30.33 & 62.11 & 39.41 & -112.68 \\
\hline Line.16 & 15.95 & -94.46 & 29.81 & -88.89 & 45.73 & 89.16 & 15.98 & 85.54 & 29.79 & 91.06 & 45.75 & -90.87 \\
\hline Line.17 & 0.03 & 83.52 & 8.64 & -95.31 & 8.61 & 84.70 & 0.01 & -96.48 & 8.63 & 84.56 & 8.62 & -95.44 \\
\hline Line.18 & 0.01 & 83.52 & 8.63 & -95.44 & 8.62 & 84.56 & 0.00 & 22.31 & 8.63 & 84.50 & 8.63 & -95.50 \\
\hline Line.19 & 16.29 & -160.93 & 21.22 & -86.35 & 30.01 & 62.09 & 16.30 & 19.16 & 21.20 & 93.59 & 30.04 & -117.95 \\
\hline Line.110 & 4.37 & -36.76 & 29.17 & -95.51 & 31.66 & 91.28 & 4.38 & 143.20 & 29.17 & 84.49 & 31.66 & -88.73 \\
\hline Line.111 & 19.10 & -171.77 & 8.89 & 62.34 & 15.64 & -19.09 & 19.10 & 8.30 & 8.89 & -117.51 & 15.66 & 160.97 \\
\hline Line. 112 & 6.39 & 87.86 & 15.07 & 168.54 & 17.28 & -32.81 & 6.39 & -92.14 & 15.07 & -11.46 & 17.28 & 147.20 \\
\hline Line.113 & 5.75 & 121.28 & 14.20 & 141.61 & 19.68 & -44.18 & 5.73 & -58.62 & 14.22 & -38.38 & 19.69 & 135.86 \\
\hline Line.114 & 17.66 & -37.00 & 0.01 & -34.87 & 17.67 & 143.00 & 17.66 & 142.97 & 0.00 & 63.43 & 17.66 & -37.03 \\
\hline
\end{tabular}




\begin{tabular}{|c|c|c|c|c|c|c|c|c|c|c|c|c|}
\hline Element & I1_1 & Ang1_1 & I1_2 & Ang1_2 & I1_3 & Ang1_3 & I2_1 & Ang2_1 & I2_2 & Ang2_2 & I2_3 & Ang2_3 \\
\hline Line. 115 & 17.33 & -174.65 & 2. 8 & -59.47 & 16.29 & 14.95 & 17.34 & 5.38 & 2.97 & 120.44 & 16.30 & -165.05 \\
\hline Line.116 & 18.21 & -5.86 & 20.31 & -171.98 & 5.10 & 67.02 & 18.21 & 174.11 & 20.32 & 8.00 & 5. 1 & -113.04 \\
\hline Line.117 & 72.89 & -19.53 & 55.5 & -173.38 & 33.56 & 113.73 & 72.89 & 160.45 & 55.56 & 6.60 & 33.56 & -66.31 \\
\hline Line.118 & 56.28 & 14.06 & 55.56 & -173.40 & 20.02 & 88.25 & 56.28 & 165.94 & 55.57 & 6.60 & 20.02 & -91.77 \\
\hline Line.119 & 20.27 & $\begin{array}{l}-173.38 \\
\end{array}$ & 3.32 & -17.69 & 17.29 & 11.15 & 20.27 & 6.69 & 3.30 & 162.43 & $1 \quad .31$ & -168.83 \\
\hline Line.120 & 75.58 & -8.63 & 58.62 & -174.74 & 23.34 & 134.44 & 75.58 & 171.36 & 58.72 & 5.25 & 23.34 & -45.59 \\
\hline Line.121 & 8.76 & $-37 . " 8$ & 0.01 & -34.77 & 8.77 & $14 \quad .73$ & 8.76 & 142.67 & 0.00 & -10843 & 8.76 & -37.33 \\
\hline Line. 122 & 68.0 & -5.10 & 58.63 & -174.75 & 14.72 & 129.46 & 68.02 & 174.89 & 58.64 & 5.24 & 14.71 & -50.59 \\
\hline Line.123 & 11.67 & 17.01 & 20.32 & -172.00 & 8.98 & -3.71 & 11.66 & -163.04 & 20.33 & 7.98 & 8.99 & 176.32 \\
\hline Line. 124 & 12.11 & 173.93 & 13.51 & 103.27 & 20.91 & -43.64 & 12.11 & -6.03 & 13.52 & -76.71 & 20.91 & 136.39 \\
\hline Line. 125 & 15.16 & -5.97 & 15.17 & -126.00 & 15.16 & 113. 9 & 15.16 & 174.00 & 15.17 & 53.98 & 15.1 & -66.04 \\
\hline LinE.126 & 53.35 & -14.55 & 42.33 & 177.30 & 14.71 & 129.41 & 53.36 & 165.43 & 42.35 & -2.71 & 14.71 & -50.67 \\
\hline Line.127 & 51.60 & 5.61 & 46.82 & -172.16 & 5.13 & 165.20 & 51.60 & -174.41 & 46.84 & 7.82 & 5.11 & -14.97 \\
\hline Line. 128 & 25.80 & -15.91 & 20.67 & 163.93 & 5.11 & 165.03 & 25.80 & 164.06 & 20.69 & -16.08 & 5.09 & -15.09 \\
\hline Line.129 & 18.30 & -89.38 & 17.60 & 74.41 & 5.09 & 164.91 & 18.32 & 90.62 & 17.60 & -105.55 & 5.08 & -15.20 \\
\hline Line.130 & 15.79 & -151.69 & 17.60 & 74.47 & 13.23 & -45.91 & 15.80 & 28.35 & 17.61 & -105.48 & 13.23 & 134.16 \\
\hline Line.131 & 17.78 & -37.52 & 0.01 & -34.63 & 17.79 & 142.48 & 17.78 & 142.46 & 0.00 & -63.43 & 17.78 & -37.54 \\
\hline Line.132 & 14.74 & -116.95 & 9.33 & 64.03 & 5.40 & 61.21 & 14.76 & 63.07 & 9.33 & -115.87 & 5.42 & -118.89 \\
\hline Line.133 & 20.94 & -173.50 & 9.33 & 64.17 & 17.80 & -19.87 & 20.95 & 6.60 & 9.34 & -115.58 & 17.83 & 160.22 \\
\hline Line.134 & 17.77 & -37.39 & 0.01 & -34.69 & 17.77 & 142.61 & 17.77 & 142.60 & 0.00 & 139.90 & 17.77 & -37.40 \\
\hline
\end{tabular}


Table 4-10: 50\% DG Penetration Power Flow

\begin{tabular}{|c|c|c|c|c|c|c|c|c|c|c|c|c|}
\hline Element & kW1_1 & kvar1_1 & kW2_1 & kvar2_1 & kW3_1 & kvar3_1 & kW1_2 & kvar1 & kW2_2 & kvar2_2 & kW3_2 & kvar3_2 \\
\hline Subxf & 320.78 & 127.15 & 255.36 & 124.40 & 300.28 & 68.91 & & 0.00 & -311.97 & -81.17 & -278.37 & -126.91 \\
\hline Xfm1 & 0.00 & 0.00 & 0.00 & 0.00 & 0.00 & 0.00 & 0.00 & 0.00 & 0.00 & 0.00 & 0.00 & 0.00 \\
\hline Line.11 & 291.08 & 106.22 & 238.41 & 114.81 & 271.84 & 63.07 & -290.29 & -105.69 & -237.95 & -114.52 & -271.30 & -62.60 \\
\hline Line. 12 & -4.06 & 34.27 & 5.07 & -8.19 & 38.30 & 21.38 & 4.08 & -34.29 & -5.07 & 8.15 & -38.27 & -21.40 \\
\hline Line.13 & 44.69 & 50.09 & 87.41 & -10.45 & 123.28 & 56.25 & -44.66 & -50.10 & -87.35 & 10.44 & -123.15 & -56.22 \\
\hline Line.14 & 249.66 & 21.34 & 145.47 & 133.16 & 109.73 & -15.02 & -248.98 & -20.92 & -145.12 & -133.05 & -109.60 & 14.93 \\
\hline Line.15 & 3.01 & 25.61 & 87.35 & -10.44 & 79.80 & 80.71 & -3.00 & -25.66 & -87.28 & 10.42 & -79.68 & -80.70 \\
\hline Line.16 & 2.30 & 43.78 & 68.77 & -49.58 & 119.57 & 53.92 & -2.26 & -43.85 & -68.66 & 49.55 & -119.31 & -53.86 \\
\hline Line.17 & 0.00 & -0.09 & 20.83 & -11.89 & 21.20 & 11.72 & 0.00 & 0.03 & -20.82 & 11.83 & -21.19 & -11.78 \\
\hline Line. 18 & 0.00 & -0.03 & 20.82 & -11.83 & 21.19 & 11.78 & 0.00 & 0.00 & -20.82 & 11.80 & -21.18 & -11.80 \\
\hline Line.19 & -39.46 & 19.41 & 47.82 & -37.66 & 54.84 & 66.67 & 39.53 & -19.46 & -47.70 & 37.62 & -54.59 & -66.67 \\
\hline Line.110 & 10.49 & 6.14 & 71.28 & -40.53 & 83.23 & 34.38 & -10.49 & -6.15 & -71.25 & 40.52 & -83.20 & -34.38 \\
\hline Line.111 & -50.02 & 13.33 & -23.59 & 2.91 & -28.65 & 32.30 & 50.10 & -13.37 & 23.60 & -2.98 & 28.70 & -32.35 \\
\hline Line. 112 & 0.70 & -18.12 & 18.51 & 39.16 & -39.90 & 26.78 & -0.70 & 18.12 & -18.50 & -39.17 & 39.90 & -26.79 \\
\hline Line.113 & -7.73 & -13.06 & -0.81 & 40.18 & -50.84 & 20.71 & 7.74 & 13.01 & 0.84 & -40.22 & 50.90 & -20.74 \\
\hline Line.114 & 41.67 & 24.50 & 0.00 & -0.02 & 43.38 & -24.53 & -41.65 & -24.51 & 0.00 & 0.00 & -43.35 & 24.51 \\
\hline Line. 115 & -45.75 & 9.79 & 5.07 & -8.13 & -5.11 & 45.93 & 45.78 & -9.81 & -5.07 & 8.10 & 5.13 & -45.95 \\
\hline Line.116 & 50.59 & -0.68 & 38.82 & 41.43 & 10.38 & 10.16 & -50.56 & 0.66 & -38.79 & -41.45 & -10.38 & -10.19 \\
\hline Line.117 & 198.39 & 21.60 & 106.30 & 91.61 & 99.22 & -25.09 & -197.90 & -21.38 & -106.07 & -91.56 & -99.11 & 25.11 \\
\hline Line.118 & 155.07 & -3.85 & 106.07 & 91.56 & 54.27 & 0.13 & -154.97 & 3.88 & -105.99 & -91.54 & -54.26 & -0.14 \\
\hline Line.119 & -53.07 & 12.86 & -1.16 & -9.42 & -8.53 & 48.15 & 53.11 & -12.91 & 1.16 & 9.36 & 8.56 & -48.20 \\
\hline Line. 120 & 208.04 & -16.75 & 107.15 & 100.97 & 62.79 & -48.01 & -207.75 & 16.88 & -107.02 & -100.93 & -62.75 & 48.00 \\
\hline Line.121 & 20.50 & 12.06 & 0.00 & -0.03 & 21.51 & -12.11 & -20.50 & -12.08 & 0.00 & 0.00 & -21.50 & 12.08 \\
\hline
\end{tabular}




\begin{tabular}{|c|c|c|c|c|c|c|c|c|c|c|c|c|}
\hline Element & kW1_1 & kvar1_1 & kW2_1 & kvar2_1 & kW3_1 & kvar3_1 & kW1_2 & kvar1 & kW2_2 & kvar2_2 & kW3_2 & kvar3_2 \\
\hline Line.122 & 187.25 & -28.94 & 107.02 & 100.96 & 41.24 & -35.89 & -187.01 & 29.04 & -106.88 & -100.93 & -41.22 & 35.87 \\
\hline Line.123 & 30.02 & -12.75 & 38.79 & 41.45 & -11.08 & 22.27 & -30.02 & 12.72 & -38.77 & -41.47 & 11.08 & -22.30 \\
\hline Line.124 & -32.11 & 0.31 & -24.41 & 28.13 & -53.81 & 22.66 & 32.13 & -0.33 & 24.42 & -28.15 & 53.85 & -22.67 \\
\hline Line. 125 & 41.34 & -0.48 & 41.97 & 0.79 & 42.73 & -0.35 & -41.33 & 0.47 & -41.96 & -0.81 & -42.72 & 0.34 \\
\hline Line. 126 & 145.29 & -3.72 & 64.23 & 75.61 & 41.22 & -35.87 & -145.05 & 3.80 & -64.12 & -75.62 & -41.19 & 35.83 \\
\hline Line. 127 & 137.75 & -53.48 & 88.64 & 71.96 & 9.18 & -35.92 & -137.46 & 53.58 & -88.47 & -71.95 & -9.16 & 35.87 \\
\hline Line.128 & 69.03 & -11.88 & 18.48 & 30.25 & 9.16 & -35.87 & -68.99 & 11.86 & -18.47 & -30.29 & -9.15 & 35.83 \\
\hline Line.129 & 6.71 & 26.12 & -45.25 & -7.70 & 9.15 & -35.83 & -6.70 & -26.16 & 45.27 & 7.66 & -9.14 & 35.79 \\
\hline Line. 130 & -34.65 & 1.76 & -45.27 & -7.64 & -34.54 & -11.36 & 34.66 & -1.80 & 45.28 & 7.61 & 34.55 & 11.32 \\
\hline Line.131 & 41.36 & 24.40 & 0.00 & -0.02 & 43.68 & -24.42 & -41.34 & -24.41 & 0.00 & 0.00 & -43.66 & 24.41 \\
\hline Line.132 & -13.16 & 37.62 & -24.51 & 3.66 & 10.47 & 12.16 & 13.20 & -37.65 & 24.53 & -3.70 & -10.47 & -12.21 \\
\hline Line.133 & -54.63 & 13.21 & -24.53 & 3.72 & -33.14 & 36.68 & 54.79 & -13.26 & 24.56 & -3.82 & 33.25 & -36.75 \\
\hline Line.134 & 41.44 & 24.44 & 0.00 & $\begin{array}{l}-0.02 \\
\end{array}$ & 43.60 & -24.47 & -41.42 & -24.46 & 0.00 & 0.00 & -43.58 & 24.46 \\
\hline
\end{tabular}

\subsubsection{5\% DG penetration case}

Table 4-11: 25\% DG penetration case voltages

\begin{tabular}{|c|c|c|c|c|c|c|c|c|c|c|c|c|c|}
\hline Bus & BasekV & Node1 & Magnitude1 & Angle1 & pu1 & Node2 & Magnitude2 & Angle2 & pu2 & Node3 & Magnitude3 & Angle3 & pu3 \\
\hline $\begin{array}{c}\text { Source } \\
\text { bus }\end{array}$ & 230.00 & 1.00 & 120104.00 & -9.60 & 0.90 & 2.00 & 122191.00 & -127.90 & 0.92 & 3.00 & 124346.00 & 110.40 & 0.94 \\
\hline 709 & 4.80 & 1.00 & 2621.70 & -13.80 & 0.95 & 2.00 & 2704.47 & -130.90 & 0.98 & 3.00 & 2781.58 & 106.20 & 1.00 \\
\hline 775 & 0.48 & 1.00 & 262.17 & -13.80 & 0.95 & 2.00 & 270.45 & -130.90 & 0.98 & 3.00 & 278.16 & 106.20 & 1.00 \\
\hline 701 & 4.80 & 1.00 & 2656.43 & -13.30 & 0.96 & 2.00 & 2731.40 & -130.70 & 0.99 & 3.00 & 2799.85 & 106.70 & 1.01 \\
\hline 702 & 4.80 & 1.00 & 2644.50 & -13.50 & 0.95 & 2.00 & 2722.83 & -130.80 & 0.98 & 3.00 & 2791.10 & 106.50 & 1.01 \\
\hline 705 & 4.80 & 1.00 & 2643.16 & -13.50 & 0.95 & 2.00 & 2721.94 & -130.80 & 0.98 & 3.00 & 2788.35 & 106.50 & 1.01 \\
\hline 713 & 4.80 & 1.00 & 2642.12 & -13.50 & 0.95 & 2.00 & 2720.63 & -130.90 & 0.98 & 3.00 & 2787.52 & 106.50 & 1.01 \\
\hline
\end{tabular}




\begin{tabular}{|c|c|c|c|c|c|c|c|c|c|c|c|c|c|}
\hline Bus & BasekV & Node1 & Magnitude1 & Angle1 & pu1 & Node2 & Magnitude2 & Angle2 & pu2 & Node3 & Magnitude3 & Angle3 & pu3 \\
\hline 703 & 4.80 & 1.00 & 2633.78 & -13.70 & 0.95 & 2.00 & 2713.78 & -130.90 & 0.98 & 3.00 & 2786.90 & 106.30 & 1.01 \\
\hline 704 & 4.80 & 1.00 & 2640.44 & -13.50 & 0.95 & 2.00 & 2717.44 & -130.90 & 0.98 & 3.00 & 2783.19 & 106.40 & 1.00 \\
\hline 720 & 4.80 & 1.00 & 2638.09 & -13.50 & 0.95 & 2.00 & 2714.90 & -131.00 & 0.98 & 3.00 & 2776.15 & 106.40 & 1.00 \\
\hline 706 & 4.80 & 1.00 & 2638.04 & -13.50 & 0.95 & 2.00 & 2714.40 & -131.00 & 0.98 & 3.00 & 2775.23 & 106.40 & 1.00 \\
\hline 725 & 4.80 & 1.00 & 2638.01 & -13.50 & 0.95 & 2.00 & 2713.86 & -131.10 & 0.98 & 3.00 & 2774.48 & 106.40 & 1.00 \\
\hline 707 & 4.80 & 1.00 & 2639.42 & -13.50 & 0.95 & 2.00 & 2709.58 & -131.20 & 0.98 & 3.00 & 2767.23 & 106.40 & 1.00 \\
\hline 722 & 4.80 & 1.00 & 2639.21 & -13.50 & 0.95 & 2.00 & 2708.81 & -131.20 & 0.98 & 3.00 & 2766.04 & 106.40 & 1.00 \\
\hline 724 & 4.80 & 1.00 & 2641.81 & -13.40 & 0.95 & 2.00 & 2710.05 & -131.20 & 0.98 & 3.00 & 2767.38 & 106.50 & 1.00 \\
\hline 714 & 4.80 & 1.00 & 2640.34 & -13.50 & 0.95 & 2.00 & 2717.04 & -130.90 & 0.98 & 3.00 & 2783.30 & 106.40 & 1.00 \\
\hline 718 & 4.80 & 1.00 & 2640.09 & -13.50 & 0.95 & 2.00 & 2715.52 & -130.90 & 0.98 & 3.00 & 2784.70 & 106.50 & 1.00 \\
\hline 712 & 4.80 & 1.00 & 2641.79 & -13.40 & 0.95 & 2.00 & 2721.94 & -130.80 & 0.98 & 3.00 & 2787.42 & 106.50 & 1.01 \\
\hline 742 & 4.80 & 1.00 & 2643.92 & -13.40 & 0.95 & 2.00 & 2721.22 & -130.90 & 0.98 & 3.00 & 2787.38 & 106.50 & 1.01 \\
\hline 727 & 4.80 & 1.00 & 2631.82 & -13.70 & 0.95 & 2.00 & 2711.88 & -130.90 & 0.98 & 3.00 & 2786.03 & 106.30 & 1.01 \\
\hline 730 & 4.80 & 1.00 & 2624.23 & -13.80 & 0.95 & 2.00 & 2706.80 & -130.90 & 0.98 & 3.00 & 2782.67 & 106.20 & 1.00 \\
\hline 731 & 4.80 & 1.00 & 2622.75 & -13.80 & 0.95 & 2.00 & 2704.26 & -130.90 & 0.98 & 3.00 & 2780.61 & 106.20 & 1.00 \\
\hline 708 & 4.80 & 1.00 & 2617.07 & -13.90 & 0.94 & 2.00 & 2700.85 & -130.90 & 0.97 & 3.00 & 2780.34 & 106.10 & 1.00 \\
\hline 732 & 4.80 & 1.00 & 2616.17 & -13.90 & 0.94 & 2.00 & 2700.85 & -130.90 & 0.97 & 3.00 & 2779.73 & 106.10 & 1.00 \\
\hline 733 & 4.80 & 1.00 & 2612.99 & -14.00 & 0.94 & 2.00 & 2697.24 & -130.90 & 0.97 & 3.00 & 2779.37 & 106.10 & 1.00 \\
\hline 744 & 4.80 & 1.00 & 2631.12 & -13.70 & 0.95 & 2.00 & 2710.61 & -130.90 & 0.98 & 3.00 & 2785.76 & 106.30 & 1.01 \\
\hline 729 & 4.80 & 1.00 & 2631.48 & -13.70 & 0.95 & 2.00 & 2710.49 & -130.90 & 0.98 & 3.00 & 2786.50 & 106.30 & 1.01 \\
\hline 728 & 4.80 & 1.00 & 2630.16 & -13.70 & 0.95 & 2.00 & 2709.67 & -130.90 & 0.98 & 3.00 & 2784.86 & 106.30 & 1.00 \\
\hline 734 & 4.80 & 1.00 & 2606.67 & -14.10 & 0.94 & 2.00 & 2692.63 & -130.90 & 0.97 & 3.00 & 2777.56 & 106.00 & 1.00 \\
\hline 737 & 4.80 & 1.00 & 2601.75 & -14.20 & 0.94 & 2.00 & 2687.24 & -130.90 & 0.97 & 3.00 & 2777.37 & 106.00 & 1.00 \\
\hline 738 & 4.80 & 1.00 & 2599.65 & -14.20 & 0.94 & 2.00 & 2685.89 & -130.80 & 0.97 & 3.00 & 2777.12 & 105.90 & 1.00 \\
\hline 711 & 4.80 & 1.00 & 2598.44 & -14.20 & 0.94 & 2.00 & 2686.43 & -130.80 & 0.97 & 3.00 & 2776.74 & 105.90 & 1.00 \\
\hline 741 & 4.80 & 1.00 & 2598.60 & -14.20 & 0.94 & 2.00 & 2686.98 & -130.80 & 0.97 & 3.00 & 2777.03 & 105.90 & 1.00 \\
\hline
\end{tabular}




\begin{tabular}{|c|c|c|c|c|c|c|c|c|c|c|c|c|c|}
\hline Bus & BasekV & Node1 & Magnitude1 & Angle1 & pu1 & Node2 & Magnitude2 & Angle2 & pu2 & Node3 & Magnitude3 & Angle3 & pu3 \\
\hline 740 & 4.80 & 1.00 & 2597.29 & -14.20 & 0.94 & 2.00 & 2686.43 & -130.80 & 0.97 & 3.00 & 2775.96 & 105.90 & 1.00 \\
\hline 710 & 4.80 & 1.00 & 2605.28 & -14.00 & 0.94 & 2.00 & 2692.81 & -130.90 & 0.97 & 3.00 & 2775.49 & 106.00 & 1.00 \\
\hline 736 & 4.80 & 1.00 & 2609.23 & -14.00 & 0.94 & 2.00 & 2693.24 & -130.90 & 0.97 & 3.00 & 2775.41 & 106.10 & 1.00 \\
\hline 735 & 4.80 & 1.00 & 2604.13 & -14.00 & 0.94 & 2.00 & 2692.81 & -130.90 & 0.97 & 3.00 & 2774.71 & 106.00 & 1.00 \\
\hline
\end{tabular}

Table 4-12: 25\% DG Penetration Case Currents

\begin{tabular}{|c|c|c|c|c|c|c|c|c|c|c|c|c|}
\hline Element & I1_1 & Ang1_1 & I1_2 & Ang1_2 & I1_3 & Ang1_3 & I2_1 & Ang2_1 & I2_2 & Ang2_2 & I2_3 & Ang2_3 \\
\hline Vsource & 4.02 & 158.46 & 3.33 & 39.01 & 3.75 & -72.14 & 4.02 & -21.54 & 3.33 & -140.99 & 3.75 & 107.86 \\
\hline Subxf & 4.02 & -21.54 & 3.33 & -140.99 & 3.75 & 107.86 & 192.55 & 158.46 & 159.53 & 39.01 & 179.77 & -72.14 \\
\hline Xfm1 & 0.00 & -103.85 & 0.00 & 139.10 & 0.00 & 16.17 & 0.00 & 90.00 & 0.00 & -165.96 & 0.00 & -165.96 \\
\hline Line.11 & 165.90 & -21.28 & 139.98 & -141.37 & 154.39 & 107.04 & 165.91 & 158.69 & 139.99 & 38.61 & 154.39 & -72.99 \\
\hline Line.12 & 12.94 & -72.15 & 9.75 & -104.36 & 21.83 & 94.09 & 12.95 & 107.83 & 9.75 & 75.57 & 21.83 & -85.94 \\
\hline Line.13 & 33.75 & -33.19 & 46.93 & -116.97 & 60.72 & 96.58 & 33.75 & 146.79 & 46.93 & 63.01 & 60.72 & -83.44 \\
\hline Line.14 & 125.87 & -13.55 & 92.98 & -157.17 & 75.09 & 119.22 & 125.87 & 166.41 & 93.01 & 22.78 & 75.07 & -60.84 \\
\hline Line.15 & 16.40 & -20.85 & 46.93 & -116.99 & 48.05 & 82.85 & 16.40 & 159.08 & 46.93 & 62.99 & 48.06 & -97.17 \\
\hline Line.16 & 13.75 & -61.35 & 39.93 & -95.46 & 51.90 & 93.08 & 13.77 & 118.57 & 39.92 & 84.50 & 51.91 & -86.96 \\
\hline Line.17 & 0.03 & 76.52 & 8.74 & -101.80 & 8.71 & 78.21 & 0.01 & -103.48 & 8.73 & 78.07 & 8.72 & -101.93 \\
\hline Line.18 & 0.01 & 76.52 & 8.73 & -101.93 & 8.72 & 78.07 & 0.00 & 33.15 & 8.73 & 78.02 & 8.73 & -101.98 \\
\hline Line.19 & 6.41 & 174.98 & 31.24 & -93.74 & 31.76 & 74.62 & 6.41 & -4.78 & 31.22 & 86.22 & 31.78 & -105.42 \\
\hline Line.110 & 4.37 & -44.24 & 29.17 & -102.04 & 31.72 & 84.66 & 4.38 & 135.72 & 29.17 & 77.96 & 31.72 & -95.34 \\
\hline Line.111 & 10.17 & 159.37 & 4.81 & -33.15 & 5.58 & -9.75 & 10.17 & -20.50 & 4.78 & 146.89 & 5.60 & 170.36 \\
\hline Line. 112 & 10.71 & 35.45 & 17.63 & -173.31 & 9.71 & -25.29 & 10.71 & -144.56 & 17.63 & 6.69 & 9.72 & 154.72 \\
\hline Line.113 & 7.40 & 43.56 & 13.67 & 165.86 & 11.54 & -46.91 & 7.38 & -136.51 & 13.68 & -14.17 & 11.55 & 133.16 \\
\hline Line.114 & 18.08 & -44.36 & 0.01 & -40.84 & 18.09 & 135.64 & 18.08 & 135.62 & 0.00 & -8.75 & 18.08 & -44.38 \\
\hline
\end{tabular}




\begin{tabular}{|c|c|c|c|c|c|c|c|c|c|c|c|c|}
\hline Element & I1_1 & Ang1_1 & I1_2 & Ang1_2 & I1_3 & Ang1_3 & I2_1 & Ang2_1 & I2_2 & Ang2_2 & I2_3 & Ang2_3 \\
\hline Line. 115 & 8.97 & 178.02 & 9.74 & -104.47 & 14.60 & 38.73 & 8.97 & -1.92 & 9.74 & 75.48 & 14.61 & -141.28 \\
\hline Line.116 & 27.78 & -8.70 & 26.10 & -161.74 & 12.67 & 102.23 & 27.78 & 171.29 & 26.10 & 18.25 & 12.67 & -77.80 \\
\hline Line.117 & 98.22 & -14.97 & 67.03 & -155.46 & 63.06 & 122.51 & 98.22 & 165.02 & 67.04 & 24.52 & 63.06 & -57.51 \\
\hline Line.118 & 83.66 & -9.09 & 67.04 & -155.48 & 46.36 & 117.78 & 83.66 & 170.90 & 67.04 & 24.51 & 46.36 & -62.23 \\
\hline Line.119 & 11.19 & 158.19 & 11.53 & -78.01 & 10.70 & 41.72 & 11.18 & -21.69 & 11.51 & 101.92 & 10.73 & -138.33 \\
\hline Line. 120 & 94.61 & -10.59 & 65.52 & -165.38 & 44.99 & 131.12 & 94.61 & 169.41 & 65.53 & 14.61 & 44.99 & -48.90 \\
\hline Line.121 & 8.98 & -44.85 & 0.01 & -40.89 & 8.99 & 135.15 & 8.99 & 135.10 & 0.00 & -129.09 & 8.99 & -44.90 \\
\hline Line.122 & 87.33 & -7.28 & 65.53 & -165.40 & 36.03 & 130.09 & 87.33 & 172.72 & 65.54 & 14.60 & 36.02 & -49.93 \\
\hline Line.123 & 21.19 & 5.64 & 26.10 & -161.75 & 7.12 & 58.79 & 21.19 & -174.39 & 26.11 & 18.23 & 7.13 & -121.27 \\
\hline Line.124 & 3.80 & 136.73 & 8.24 & 132.09 & 12.03 & -46.46 & 3.80 & -43.16 & 8.24 & -47.90 & 12.03 & 133.58 \\
\hline Line. 125 & 15.49 & -12.73 & 15.52 & -132.73 & 15.50 & 107.19 & 15.49 & 167.25 & 15.52 & 47.25 & 15.50 & -72.83 \\
\hline Line.126 & 71.74 & -13.36 & 47.88 & -166.71 & 36.02 & 130.07 & 71.74 & 166.62 & 47.89 & 13.27 & 36.01 & -49.96 \\
\hline Line.127 & 62.09 & 2.50 & 48.98 & -166.46 & 16.85 & 148.73 & 62.08 & -177.52 & 48.99 & 13.52 & 16.84 & -31.33 \\
\hline Line.128 & 34.88 & -10.50 & 20.05 & -173.10 & 16.84 & 148.67 & 34.87 & 169.47 & 20.06 & 6.87 & 16.82 & -31.37 \\
\hline Line.129 & 17.02 & -60.54 & 8.52 & 45.22 & 16.82 & 148.63 & 17.03 & 119.43 & 8.52 & -134.68 & 16.81 & -31.41 \\
\hline Line.130 & 4.86 & -157.30 & 8.52 & 45.36 & 4.45 & -109.55 & 4.87 & 22.84 & 8.52 & -134.54 & 4.45 & 70.61 \\
\hline Line.131 & 18.25 & -45.23 & 0.01 & -40.84 & 18.26 & 134.77 & 18.25 & 134.76 & 0.00 & -108.00 & 18.25 & -45.24 \\
\hline Line.132 & 13.01 & -83.79 & 1.11 & 25.31 & 12.68 & 100.94 & 13.02 & 96.19 & 1.10 & -153.93 & 12.68 & -79.13 \\
\hline Line.133 & 11.48 & -179.81 & 1.10 & 26.37 & 10.51 & -2.51 & 11.49 & 0.38 & 1.09 & -151.72 & 10.55 & 177.56 \\
\hline Line.134 & 18.24 & -45.05 & 0.01 & -40.87 & 18.25 & 134.95 & 18.24 & 134.93 & 0.00 & -115.99 & 18.24 & -45.07 \\
\hline
\end{tabular}


Table 4-13: 25\% DG Penetration Power Flow

\begin{tabular}{|c|c|c|c|c|c|c|c|c|c|c|c|c|}
\hline Element & kW1_1 & kvar1_1 & kW2_1 & kvar2_1 & kW3_1 & kvar3_1 & kW1_2 & kvar1 & kW2_2 & kvar2_2 & kW3_2 & kvar3_2 \\
\hline Subxf & 507.31 & 486.55 & 472.84 & 488.09 & 456.32 & 515.44 & 0.00 & 0.00 & -394.41 & -108.58 & -411.53 & -107.89 \\
\hline Xfm1 & 0.00 & 0.00 & 0.00 & 0.00 & 0.00 & 0.00 & 0.00 & 0.00 & 0.00 & 0.00 & 0.00 & 0.00 \\
\hline Line.11 & 347.46 & 60.01 & 344.78 & 65.07 & 332.18 & 78.44 & -321.82 & -37.75 & -323.25 & -42.45 & -310.44 & -51.58 \\
\hline Line. 12 & -12.34 & -2.93 & -12.43 & -4.42 & -10.69 & -4.10 & 12.42 & 2.95 & 12.51 & 4.44 & 10.75 & 4.12 \\
\hline Line.13 & -21.26 & -7.55 & -10.24 & -16.11 & -7.77 & -2.62 & 21.37 & 7.61 & 10.32 & 16.14 & 7.79 & 2.63 \\
\hline Line.14 & 355.42 & 48.22 & 345.91 & 62.97 & 328.91 & 58.30 & -312.41 & -10.57 & -311.47 & -26.78 & -295.24 & -16.46 \\
\hline Line. 15 & -23.01 & -8.52 & -10.32 & -16.14 & -9.38 & -1.71 & 23.20 & 8.62 & 10.43 & 16.20 & 9.40 & 1.73 \\
\hline Line.16 & -11.84 & -3.03 & -1.07 & -13.18 & 2.61 & 1.24 & 11.91 & 3.06 & 1.15 & 13.22 & -2.60 & -1.24 \\
\hline Line.17 & 0.00 & 0.00 & 0.78 & -0.47 & 0.76 & 0.46 & 0.00 & 0.00 & -0.78 & 0.46 & -0.76 & -0.46 \\
\hline Line.18 & 0.00 & 0.00 & 0.78 & -0.46 & 0.76 & 0.46 & 0.00 & 0.00 & -0.78 & 0.46 & -0.76 & -0.46 \\
\hline Line.19 & -13.59 & -3.99 & -1.93 & -12.75 & 0.24 & 1.71 & 13.79 & 4.05 & 2.10 & 12.80 & -0.24 & -1.71 \\
\hline Line. 110 & 2.05 & 1.11 & 12.90 & -7.79 & 14.38 & 6.69 & -2.05 & -1.11 & -12.86 & 7.80 & -14.35 & -6.68 \\
\hline Line.111 & -15.84 & -5.17 & -15.00 & -5.01 & -14.15 & -4.98 & 16.07 & 5.23 & 15.21 & 5.07 & 14.34 & 5.05 \\
\hline Line.112 & -11.36 & -5.60 & -9.36 & -3.02 & -12.01 & -2.97 & 11.37 & 5.60 & 9.37 & 3.03 & 12.02 & 2.97 \\
\hline Line.113 & -12.98 & -4.70 & -12.87 & -2.78 & -13.92 & -4.12 & 13.10 & 4.73 & 12.97 & 2.81 & 14.05 & 4.17 \\
\hline Line.114 & 1.65 & 0.92 & 0.00 & 0.00 & 1.60 & -0.92 & -1.64 & -0.92 & 0.00 & 0.00 & -1.60 & 0.92 \\
\hline Line. 115 & -14.06 & -3.87 & -12.51 & -4.44 & -12.35 & -3.21 & 14.14 & 3.89 & 12.57 & 4.46 & 12.41 & 3.23 \\
\hline Line.116 & -7.59 & -4.47 & -8.22 & -0.17 & -11.53 & -2.84 & 7.62 & 4.48 & 8.25 & 0.17 & 11.58 & 2.85 \\
\hline Line. 117 & 320.00 & 15.03 & 319.70 & 26.94 & 306.77 & 19.30 & -269.50 & 11.89 & -272.70 & -2.62 & -265.32 & 8.55 \\
\hline Line.118 & -16.21 & -12.35 & -20.38 & 2.62 & -32.00 & -8.08 & 16.31 & 12.41 & 20.47 & -2.58 & 32.22 & 8.22 \\
\hline Line.119 & -13.20 & -2.27 & -12.44 & -3.00 & -12.65 & -1.77 & 13.32 & 2.34 & 12.54 & 3.05 & 12.74 & 1.84 \\
\hline Line. 120 & -3.11 & -10.14 & -8.03 & 5.57 & -19.57 & -6.45 & 3.15 & 10.16 & 8.06 & -5.56 & 19.71 & 6.53 \\
\hline Line. 121 & 0.44 & 0.26 & 0.00 & 0.00 & 0.46 & -0.26 & -0.44 & -0.26 & 0.00 & 0.00 & -0.46 & 0.26 \\
\hline Line.122 & -3.60 & -10.42 & -8.06 & 5.56 & -20.17 & -6.27 & 3.64 & 10.45 & 8.09 & -5.54 & 20.32 & 6.35 \\
\hline
\end{tabular}




\begin{tabular}{|c|c|c|c|c|c|c|c|c|c|c|c|c|}
\hline Element & kW1_1 & kvar1_1 & kW2_1 & kvar2_1 & kW3_1 & kvar3_1 & kW1_2 & kvar1 & kW2_2 & kvar2_2 & kW3_2 & kvar3_2 \\
\hline Line.123 & -8.23 & -4.83 & -8.25 & -0.17 & -12.19 & -2.50 & 8.25 & 4.84 & 8.26 & 0.18 & 12.22 & 2.52 \\
\hline Line.124 & -10.10 & -4.48 & -10.12 & -0.54 & -13.45 & -2.52 & 10.15 & 4.50 & 10.16 & 0.55 & 13.52 & 2.55 \\
\hline Line.125 & 1.23 & 0.00 & 1.24 & 0.00 & 1.23 & 0.00 & -1.23 & 0.00 & -1.24 & 0.00 & -1.22 & 0.00 \\
\hline Line.126 & -9.44 & -6.96 & -13.96 & 2.05 & -20.32 & -6.35 & 9.53 & 7.01 & 14.08 & -1.99 & 20.57 & 6.51 \\
\hline Line.127 & 1.28 & -6.57 & -2.37 & 3.96 & -9.90 & -4.57 & -1.25 & 6.59 & 2.38 & -3.95 & 9.98 & 4.61 \\
\hline Line.128 & -8.38 & -0.71 & -12.08 & -1.93 & -9.98 & -4.61 & 8.41 & 0.72 & 12.14 & 1.96 & 10.02 & 4.64 \\
\hline Line.129 & -9.74 & 0.09 & -13.49 & -2.77 & -10.02 & -4.64 & 9.78 & -0.07 & 13.56 & 2.81 & 10.07 & 4.67 \\
\hline Line.130 & -10.74 & -0.48 & -13.56 & -2.81 & -11.07 & -4.11 & 10.79 & 0.51 & 13.64 & 2.85 & 11.12 & 4.14 \\
\hline Line.131 & 0.95 & 0.56 & 0.00 & 0.00 & 1.01 & -0.56 & -0.95 & -0.56 & 0.00 & 0.00 & -1.01 & 0.56 \\
\hline Line.132 & -11.27 & -0.71 & -11.71 & -1.96 & -11.14 & -1.67 & 11.41 & 0.75 & 11.86 & 2.01 & 11.26 & 1.71 \\
\hline Line.133 & -12.36 & -1.31 & -11.86 & -2.01 & -12.26 & -1.16 & 12.76 & 1.43 & 12.21 & 2.11 & 12.60 & 1.28 \\
\hline Line.134 & 0.95 & 0.55 & 0.00 & 0.00 & 0.99 & -0.55 & -0.95 & -0.55 & 0.00 & 0.00 & -0.99 & 0.55 \\
\hline
\end{tabular}




\subsection{Fault Location Algorithm}

There are basically two stages in the fault location. They are 1) identifying the type of fault whether the fault is single line to ground, line to line or three phase to ground and 2) determining the location of the fault.

\subsubsection{Identifying Fault Type}

One of the most important aspects of the fault location algorithm is identifying the fault type. Each of the fault types considered, line to line (LL), single line to ground (SLG) and three phase to ground (3LG), can be identified based on the magnitudes of the sequence components of the current. The different sequence components of current are positive sequence current (I1), negative sequence current (I2) and the zero sequence current (I0). Each of these components shows violation depending on the kind of fault occurring in the system. For identifying SLG, 3LG and LL faults the residual current (Ir), positive sequence current (I1) and negative sequence current (I2) magnitudes respectively are used. The residual current is the sum of positive, negative and zero sequence currents. The reason for using sequence current magnitudes for fault detection is due to the fact that when a fault occurs, depending on its type, the corresponding sequence current magnitudes are high in the affected lines; thus the sequence current magnitudes give a rough estimate of where the fault exists.

$$
\begin{aligned}
& \mathrm{I}_{1}=\frac{1}{3} *\left(\mathrm{I}_{\mathrm{a}}+\lambda * \mathrm{I}_{\mathrm{b}}+\lambda^{2} * \mathrm{I}_{\mathrm{c}}\right) \\
& \mathrm{I}_{2}=\frac{1}{3} *\left(\mathrm{I}_{\mathrm{a}}+\lambda^{2} \mathrm{I}_{\mathrm{b}}+\lambda * \mathrm{I}_{\mathrm{c}}\right) \\
& \mathrm{I}_{0}=\frac{1}{3} *\left(\mathrm{I}_{\mathrm{a}}+\mathrm{I}_{\mathrm{b}}+\mathrm{I}_{\mathrm{c}}\right) \\
& \text { Where } \quad \lambda=1120
\end{aligned}
$$

The thresholds ( $\mathrm{I}_{\mathrm{r}}$ th, $\mathrm{I}_{1}$ th, and $\mathrm{I}_{2}$ th) are set for these sequence currents and all the lines violating the limit are suspected of having a fault in them. So, the Switch Agent first checks all the lines that exceed these thresholds.

In order to determine these thresholds, a detailed short circuit analysis of the test system was performed. The detailed short circuit study for each of these penetration levels is provided in the section 4.4 . 


\subsubsection{Determining The Fault Location}

The next phase of the fault location algorithm is determining the fault location. Determination of the fault location is dependent on the location of the node, that is, whether it has generation sources on either side or only on one side (unidirectional flow).

For a node with generation sources on either side of it, the fault location can be determined by checking the current direction in all the lines that are connected to the violating line. The basic reason for checking for current direction for such a node is due to the simple fact that all the generation units on either side of the node will feed this node during a fault. Thus, the adjoining lines of such a node will have current flowing towards the faulty node and in the opposite direction. This is considered as the current reversal condition and the fault can be located based on it.

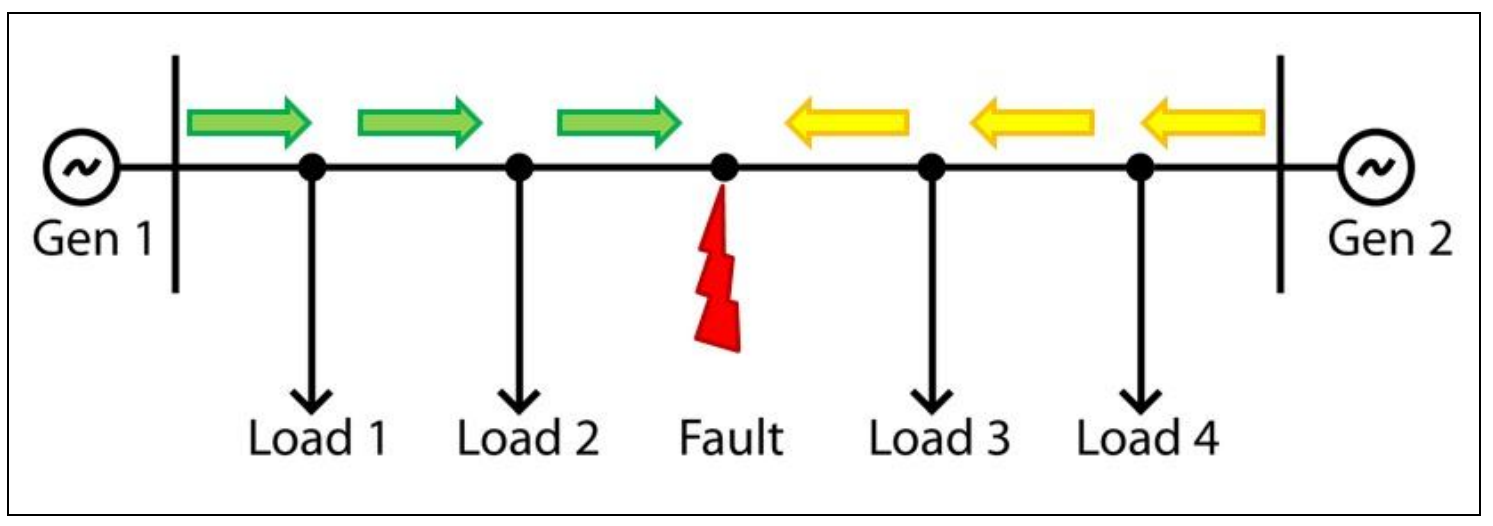

Figure 4.4: Power flow during fault

The current direction for an SLG fault is determined by looking at the phase angle of the zero sequence current. For a SLG fault at a node with generation sources on either side, the phase angle of the zero sequence current in the adjoining lines of the node will be of opposite sign. While for multiple phase faults like 3LG and LL, the current direction can be determined from the values of real power. For a $3 \mathrm{LG}$ fault, the real power values will be of opposite signs in all the 3 phases; while for a LL fault, this would be the case in the two faulted phases in the lines attached to the faulty node. To add further credibility for the determination of fault location in case of $3 \mathrm{LG}$ and LL faults, the phase angles of the 
corresponding sequence components can be used. Similar to SLG fault, these phase angles in the adjoining lines of the faulty node will be of opposite sign.

In the scenario where the fault occurs at a node with unidirectional power flow, the condition for current reversal will not be satisfied. The sequence component current value in the line above the faulty node will exceed any of the thresholds $\mathrm{I}_{1}$ th, $\mathrm{I}_{2}$ th and $\mathrm{I}_{\mathrm{r}}$ th depending on the type of the fault, and the line below will have a low value of the residual current.

The complete flow chart of the fault location algorithm is provided in Figure 4-5. As seen in the flow chart, first the conditions for current reversal are checked. When these conditions are not true, the condition for negligible residual current is checked. 


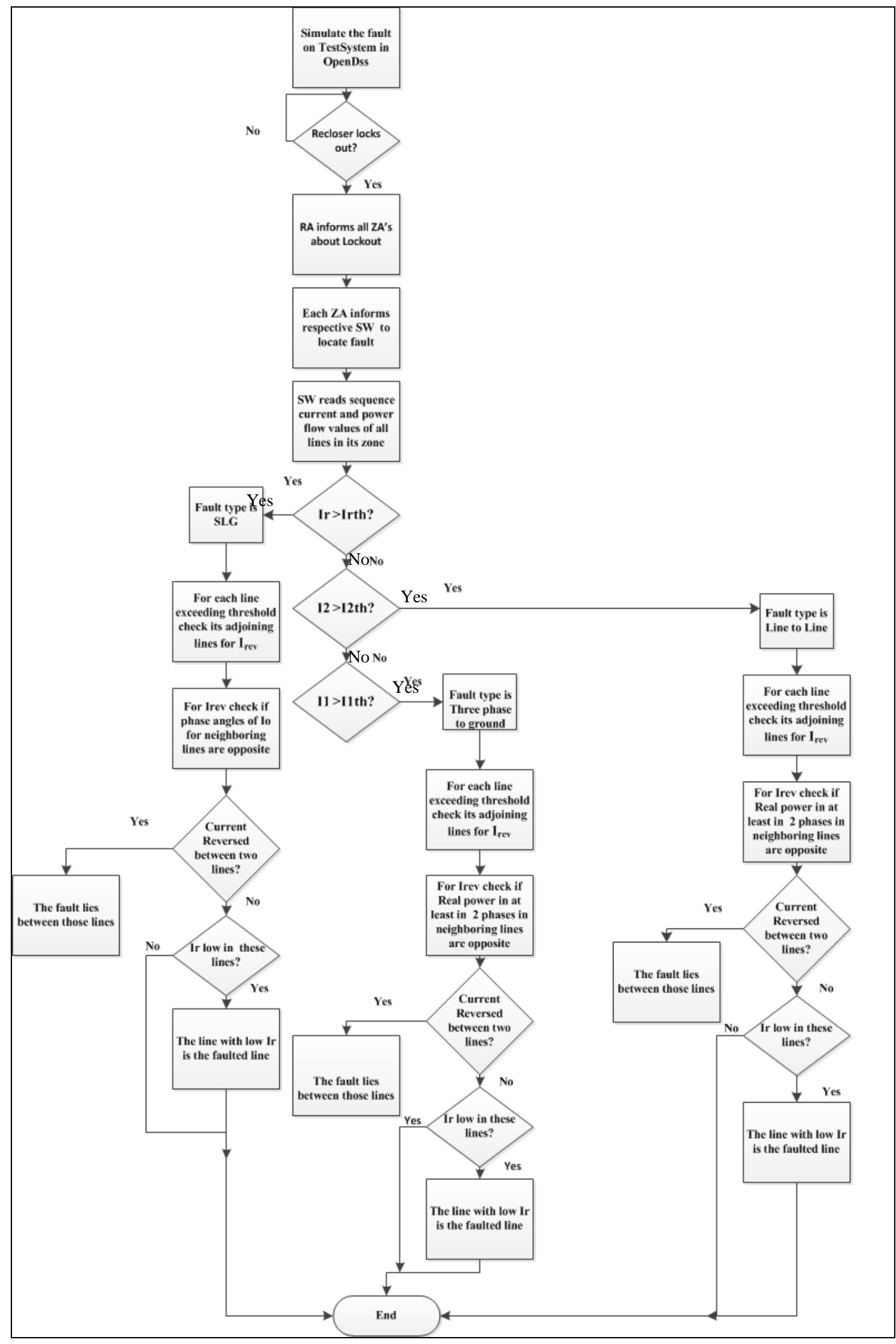

Figure 4-5: Flow Chart for fault Location 


\subsection{Short Circuit Analysis:}

In this section, the results of the short circuit analysis for the different penetration levels of the Distributed Generation in the Microgrid are presented. All the fault types considered in this work (LL, SLG and 3LG) are simulated at various nodes in each of the zones of the test system. The values of I1 for 3LG, Ir for SLG and I2 for LL fault in all the distribution lines in the system have been tabulated. Based on these values I1th, I2th and Irth for each of zones have been determined. The values of these thresholds for the Switch Agent vary for the each of the zones in the test system. The resistance of the fault is considered as $0.5 \mathrm{ohm}$.

\subsubsection{Short Circuit Analysis 50\% DG Penetration}

Table 4-14: $I_{1}$ value, zone 1,3 phase fault, $50 \%$ penetration

\begin{tabular}{|c|c|c|c|c|c|c|c|c|}
\hline Element & Terminal & \multicolumn{9}{|c|}{ Faulty Node } \\
\hline & & $\mathbf{7 0 5}$ & $\mathbf{7 1 3}$ & $\mathbf{7 0 4}$ & $\mathbf{7 1 4}$ & $\mathbf{7 2 0}$ & $\mathbf{7 0 6}$ & $\mathbf{7 0 7}$ \\
\hline Vsource & 1 & 17.15 & 17.36 & 16.95 & 16.85 & 16.32 & 15.86 & 15.33 \\
\hline Vsource & 2 & 17.15 & 17.36 & 16.95 & 16.85 & 16.32 & 15.86 & 15.33 \\
\hline Subxf & 1 & 17.15 & 17.36 & 16.95 & 16.85 & 16.32 & 15.86 & 15.33 \\
\hline Subxf & 2 & 822.00 & 831.65 & 812.04 & 807.52 & 781.96 & 760.06 & 734.56 \\
\hline Xfm1 & 1 & 0.00 & 0.00 & 0.00 & 0.00 & 0.00 & 0.00 & 0.00 \\
\hline Xfm1 & 2 & 0.00 & 0.00 & 0.00 & 0.00 & 0.00 & 0.00 & 0.00 \\
\hline Line.11 & 1 & 745.10 & 754.06 & 736.04 & 731.87 & 708.40 & 688.29 & 664.72 \\
\hline Line.11 & 2 & 745.10 & 754.06 & 736.04 & 731.87 & 708.40 & 688.29 & 664.72 \\
\hline Line.12 & 1 & 1037.53 & 56.58 & 54.74 & 54.29 & 51.92 & 49.89 & 47.33 \\
\hline Line.12 & 2 & 1037.53 & 56.58 & 54.74 & 54.29 & 51.92 & 49.88 & 47.33 \\
\hline Line.13 & 1 & 94.48 & 1013.47 & 985.86 & 979.35 & 943.62 & 912.94 & 875.77 \\
\hline Line.13 & 2 & 94.48 & 1013.47 & 985.86 & 979.35 & 943.62 & 912.94 & 875.77 \\
\hline Line.14 & 1 & 199.49 & 203.93 & 196.33 & 194.44 & 184.75 & 176.36 & 165.17 \\
\hline Line.14 & 2 & 199.49 & 203.93 & 196.33 & 194.44 & 184.75 & 176.35 & 165.16 \\
\hline Line.15 & 1 & 97.22 & 101.53 & 983.32 & 976.73 & 940.68 & 909.70 & 871.99 \\
\hline Line.15 & 2 & 97.21 & 101.53 & 983.32 & 976.73 & 940.68 & 909.71 & 871.99 \\
\hline Line.16 & 1 & 43.63 & 45.99 & 46.21 & 45.78 & 993.63 & 960.86 & 921.13 \\
\hline Line.16 & 2 & 43.63 & 45.99 & 46.21 & 45.78 & 993.63 & 960.86 & 921.13 \\
\hline Line.17 & 1 & 1.39 & 1.15 & 1.11 & 1.15 & 1.06 & 1005.05 & 1.50 \\
\hline Line.17 & 2 & 1.39 & 1.15 & 1.11 & 1.15 & 1.06 & 1005.05 & 1.50 \\
\hline Line.18 & 1 & 1.39 & 1.15 & 1.11 & 1.15 & 1.06 & 1.02 & 1.50 \\
\hline & & & & & & & & \\
\hline
\end{tabular}




\begin{tabular}{|c|c|c|c|c|c|c|c|c|}
\hline \multirow[t]{2}{*}{ Element } & \multirow[t]{2}{*}{ Terminal } & \multicolumn{7}{|c|}{ Faulty Node } \\
\hline & & 705 & 713 & 704 & 714 & 720 & 706 & 707 \\
\hline Line.18 & 2 & 1.39 & 1.15 & 1.11 & 1.15 & 1.06 & 1.02 & 1.50 \\
\hline Line.19 & 1 & 47.74 & 49.42 & 49.50 & 49.18 & 49.55 & 48.08 & 916.64 \\
\hline Line.19 & 2 & 47.74 & 49.42 & 49.50 & 49.18 & 49.55 & 48.08 & 916.64 \\
\hline Line. 110 & 1 & 19.36 & 19.36 & 19.37 & 19.37 & 19.37 & 19.36 & 19.37 \\
\hline Line.110 & 2 & 19.36 & 19.36 & 19.37 & 19.37 & 19.37 & 19.36 & 19.37 \\
\hline Line. 111 & 1 & 66.81 & 68.61 & 68.65 & 68.29 & 68.60 & 66.72 & 68.33 \\
\hline Line.111 & 2 & 66.80 & 68.61 & 68.65 & 68.29 & 68.60 & 66.71 & 68.33 \\
\hline Line.112 & 1 & 53.75 & 55.65 & 55.71 & 1021.59 & 53.22 & 51.41 & 49.34 \\
\hline Line.112 & 2 & 53.75 & 55.65 & 55.71 & 1021.59 & 53.22 & 51.41 & 49.34 \\
\hline Line. 113 & 1 & 58.30 & 60.21 & 60.27 & 60.24 & 57.71 & 55.85 & 53.78 \\
\hline Line.113 & 2 & 58.29 & 60.21 & 60.27 & 60.24 & 57.71 & 55.84 & 53.78 \\
\hline Line.114 & 1 & 2.23 & 2.53 & 2.81 & 2.90 & 3.24 & 3.56 & 4.09 \\
\hline Line.114 & 2 & 2.23 & 2.53 & 2.81 & 2.90 & 3.24 & 3.56 & 4.09 \\
\hline Line. 115 & 1 & 60.08 & 59.10 & 57.51 & 57.15 & 55.08 & 53.32 & 51.29 \\
\hline Line.115 & 2 & 60.08 & 59.10 & 57.51 & 57.15 & 55.08 & 53.32 & 51.29 \\
\hline Line.116 & 1 & 59.14 & 60.35 & 58.33 & 57.83 & 55.26 & 53.03 & 50.03 \\
\hline Line.116 & 2 & 59.14 & 60.35 & 58.33 & 57.83 & 55.26 & 53.03 & 50.03 \\
\hline Line.117 & 1 & 140.46 & 143.68 & 138.08 & 136.70 & 129.57 & 123.40 & 115.23 \\
\hline Line.117 & 2 & 140.46 & 143.68 & 138.08 & 136.70 & 129.57 & 123.40 & 115.23 \\
\hline Line.118 & 1 & 143.06 & 146.06 & 140.69 & 139.38 & 132.50 & 126.56 & 118.86 \\
\hline Line.118 & 2 & 143.06 & 146.06 & 140.69 & 139.38 & 132.50 & 126.56 & 118.86 \\
\hline Line.119 & 1 & 60.96 & 61.72 & 60.12 & 59.76 & 57.67 & 55.89 & 53.87 \\
\hline Line.119 & 2 & 60.96 & 61.72 & 60.12 & 59.76 & 57.67 & 55.89 & 53.87 \\
\hline Line. 120 & 1 & 82.12 & 84.36 & 80.62 & 79.68 & 74.96 & 70.88 & 65.21 \\
\hline Line. 120 & 2 & 82.11 & 84.36 & 80.62 & 79.68 & 74.96 & 70.88 & 65.20 \\
\hline Line. 121 & 1 & 1.46 & 1.34 & 1.48 & 1.52 & 1.69 & 1.84 & 2.10 \\
\hline Line.121 & 2 & 1.46 & 1.34 & 1.48 & 1.52 & 1.69 & 1.84 & 2.10 \\
\hline Line.122 & 1 & 83.56 & 85.68 & 82.06 & 81.16 & 76.57 & 72.60 & 67.18 \\
\hline Line.122 & 2 & 83.56 & 85.68 & 82.06 & 81.16 & 76.57 & 72.60 & 67.18 \\
\hline Line.123 & 1 & 60.52 & 61.62 & 59.72 & 59.25 & 56.82 & 54.70 & 51.95 \\
\hline Line.123 & 2 & 60.52 & 61.62 & 59.72 & 59.25 & 56.82 & 54.70 & 51.95 \\
\hline Line.124 & 1 & 66.06 & 66.69 & 65.25 & 64.94 & 63.04 & 61.43 & 59.67 \\
\hline Line. 124 & 2 & 66.06 & 66.69 & 65.25 & 64.93 & 63.04 & 61.43 & 59.66 \\
\hline Line. 125 & 1 & 4.23 & 3.87 & 4.28 & 4.40 & 4.90 & 5.36 & 6.14 \\
\hline Line. 125 & 2 & 4.23 & 3.87 & 4.28 & 4.40 & 4.90 & 5.36 & 6.14 \\
\hline Line.126 & 1 & 93.68 & 95.81 & 92.08 & 91.17 & 86.41 & 82.31 & 76.91 \\
\hline Line. 126 & 2 & 93.68 & 95.81 & 92.08 & 91.16 & 86.41 & 82.30 & 76.91 \\
\hline Line.127 & 1 & 35.00 & 36.13 & 34.35 & 33.89 & 31.68 & 29.75 & 26.91 \\
\hline Line.127 & 2 & 35.00 & 36.13 & 34.35 & 33.89 & 31.67 & 29.75 & 26.91 \\
\hline
\end{tabular}




\begin{tabular}{|c|c|c|c|c|c|c|c|c|}
\hline Element & Terminal & \multicolumn{7}{|c|}{ Faulty Node } \\
\hline & & $\mathbf{7 0 5}$ & $\mathbf{7 1 3}$ & $\mathbf{7 0 4}$ & $\mathbf{7 1 4}$ & $\mathbf{7 2 0}$ & $\mathbf{7 0 6}$ & $\mathbf{7 0 7}$ \\
\hline Line.128 & 1 & 51.64 & 52.76 & 50.79 & 50.31 & 47.79 & 45.61 & 42.81 \\
\hline Line.128 & 2 & 51.63 & 52.76 & 50.79 & 50.31 & 47.79 & 45.61 & 42.81 \\
\hline Line.129 & 1 & 55.99 & 56.76 & 55.16 & 54.79 & 52.71 & 50.94 & 48.87 \\
\hline Line.129 & 2 & 55.99 & 56.76 & 55.16 & 54.79 & 52.71 & 50.94 & 48.87 \\
\hline Line.130 & 1 & 59.02 & 59.56 & 58.22 & 57.93 & 56.15 & 54.64 & 53.10 \\
\hline Line.130 & 2 & 59.02 & 59.56 & 58.22 & 57.93 & 56.15 & 54.64 & 53.10 \\
\hline Line.131 & 1 & 3.06 & 2.83 & 3.10 & 3.18 & 3.52 & 3.83 & 4.34 \\
\hline Line.131 & 2 & 3.06 & 2.83 & 3.10 & 3.18 & 3.52 & 3.83 & 4.34 \\
\hline Line.132 & 1 & 60.23 & 61.12 & 59.37 & 58.96 & 56.68 & 54.74 & 52.45 \\
\hline Line.132 & 2 & 60.23 & 61.12 & 59.36 & 58.96 & 56.68 & 54.74 & 52.45 \\
\hline Line.133 & 1 & 63.27 & 63.92 & 62.42 & 62.09 & 60.12 & 58.46 & 56.69 \\
\hline Line.133 & 2 & 63.27 & 63.91 & 62.42 & 62.09 & 60.12 & 58.45 & 56.69 \\
\hline Line.134 & 1 & 3.05 & 2.81 & 3.09 & 3.17 & 3.51 & 3.82 & 4.33 \\
\hline Line.134 & 2 & 3.05 & 2.81 & 3.09 & 3.17 & 3.51 & 3.82 & 4.33 \\
\hline
\end{tabular}

Table 4-15: $I_{\mathrm{r}}$ value, zone 1, SLG fault, $50 \%$ penetration

\begin{tabular}{|c|c|c|c|c|c|c|c|c|}
\hline Element & Terminal & \multicolumn{7}{|c|}{ Faulty Node } \\
\hline & & $\mathbf{7 0 5}$ & $\mathbf{7 1 3}$ & $\mathbf{7 0 4}$ & $\mathbf{7 1 4}$ & $\mathbf{7 2 0}$ & $\mathbf{7 0 6}$ & $\mathbf{7 0 7}$ \\
\hline Vsource & 1 & 0.00 & 0.00 & 0.00 & 0.00 & 0.00 & 0.00 & 0.00 \\
\hline Vsource & 2 & 0.00 & 0.00 & 0.00 & 0.00 & 0.00 & 0.00 & 0.00 \\
\hline Subxf & 1 & 0.00 & 0.00 & 0.00 & 0.00 & 0.00 & 0.00 & 0.00 \\
\hline Subxf & 2 & 0.00 & 0.00 & 0.00 & 0.00 & 0.00 & 0.00 & 0.00 \\
\hline Xfm1 & 1 & 0.00 & 0.00 & 0.00 & 0.00 & 0.00 & 0.00 & 0.00 \\
\hline Xfm1 & 2 & 0.00 & 0.00 & 0.00 & 0.00 & 0.00 & 0.00 & 0.00 \\
\hline Line.11 & 1 & 0.22 & 0.22 & 0.21 & 0.21 & 0.20 & 0.20 & 0.19 \\
\hline Line.11 & 2 & 0.22 & 0.22 & 0.21 & 0.21 & 0.20 & 0.20 & 0.19 \\
\hline Line.12 & 1 & 795.93 & 132.91 & 128.95 & 128.04 & 122.42 & 117.38 & 112.96 \\
\hline Line.12 & 2 & 795.93 & 132.91 & 128.95 & 128.04 & 122.42 & 117.38 & 112.96 \\
\hline Line.13 & 1 & 257.83 & 678.75 & 658.49 & 653.86 & 625.13 & 599.41 & 576.85 \\
\hline Line.13 & 2 & 257.83 & 678.75 & 658.49 & 653.86 & 625.13 & 599.41 & 576.85 \\
\hline Line.14 & 1 & 538.54 & 546.47 & 530.16 & 526.43 & 503.30 & 482.59 & 464.43 \\
\hline Line.14 & 2 & 538.54 & 546.47 & 530.16 & 526.43 & 503.30 & 482.59 & 464.43 \\
\hline Line.15 & 1 & 257.85 & 266.79 & 658.47 & 653.84 & 625.11 & 599.40 & 576.83 \\
\hline Line.15 & 2 & 257.85 & 266.79 & 658.47 & 653.84 & 625.11 & 599.40 & 576.83 \\
\hline Line.16 & 1 & 128.42 & 132.87 & 132.65 & 131.72 & 751.26 & 720.35 & 693.24 \\
\hline Line.16 & 2 & 128.42 & 132.87 & 132.65 & 131.72 & 751.26 & 720.35 & 693.24 \\
\hline Line.17 & 1 & 0.04 & 0.05 & 0.05 & 0.05 & 0.05 & 845.69 & 0.04 \\
\hline Line.17 & 2 & 0.04 & 0.05 & 0.05 & 0.05 & 0.05 & 845.69 & 0.04 \\
\hline & & & & & & & & \\
\hline
\end{tabular}




\begin{tabular}{|c|c|c|c|c|c|c|c|c|}
\hline \multirow[t]{2}{*}{ Element } & \multirow[t]{2}{*}{ Terminal } & \multicolumn{7}{|c|}{ Faulty Node } \\
\hline & & 705 & 713 & 704 & 714 & 720 & 706 & 707 \\
\hline Line.18 & 1 & 0.01 & 0.01 & 0.01 & 0.01 & 0.01 & 0.01 & 0.01 \\
\hline Line. 18 & 2 & 0.01 & 0.01 & 0.01 & 0.01 & 0.01 & 0.01 & 0.01 \\
\hline Line.19 & 1 & 128.51 & 132.96 & 132.74 & 131.81 & 131.91 & 126.48 & 693.16 \\
\hline Line.19 & 2 & 128.51 & 132.96 & 132.74 & 131.81 & 131.91 & 126.48 & 693.16 \\
\hline Line.110 & 1 & 0.01 & 0.01 & 0.01 & 0.01 & 0.01 & 0.01 & 0.01 \\
\hline Line.110 & 2 & 0.01 & 0.01 & 0.01 & 0.01 & 0.01 & 0.01 & 0.01 \\
\hline Line.111 & 1 & 128.55 & 133.01 & 132.79 & 131.86 & 131.95 & 126.53 & 130.56 \\
\hline Line.111 & 2 & 128.55 & 133.01 & 132.79 & 131.86 & 131.95 & 126.53 & 130.56 \\
\hline Line.112 & 1 & 129.50 & 133.99 & 133.77 & 785.07 & 126.99 & 121.77 & 117.19 \\
\hline Line.112 & 2 & 129.50 & 133.99 & 133.77 & 785.07 & 126.99 & 121.77 & 117.19 \\
\hline Line.113 & 1 & 129.50 & 133.99 & 133.77 & 133.58 & 127.00 & 121.77 & 117.19 \\
\hline Line.113 & 2 & 129.50 & 133.99 & 133.77 & 133.58 & 127.00 & 121.77 & 117.19 \\
\hline Line.114 & 1 & 0.01 & 0.01 & 0.01 & 0.01 & 0.01 & 0.01 & 0.01 \\
\hline Line.114 & 2 & 0.01 & 0.01 & 0.01 & 0.01 & 0.01 & 0.01 & 0.01 \\
\hline Line.115 & 1 & 134.65 & 132.94 & 128.97 & 128.07 & 122.44 & 117.41 & 112.99 \\
\hline Line.115 & 2 & 134.65 & 132.94 & 128.97 & 128.07 & 122.44 & 117.41 & 112.99 \\
\hline Line.116 & 1 & 136.69 & 138.70 & 134.56 & 133.61 & 127.75 & 122.49 & 117.88 \\
\hline Line.116 & 2 & 136.69 & 138.70 & 134.56 & 133.61 & 127.75 & 122.49 & 117.88 \\
\hline Line.117 & 1 & 402.22 & 408.14 & 395.95 & 393.17 & 375.89 & 360.43 & 346.86 \\
\hline Line.117 & 2 & 402.22 & 408.14 & 395.95 & 393.17 & 375.89 & 360.43 & 346.86 \\
\hline Line.118 & 1 & 402.25 & 408.17 & 395.98 & 393.20 & 375.92 & 360.46 & 346.89 \\
\hline Line.118 & 2 & 402.25 & 408.17 & 395.98 & 393.20 & 375.92 & 360.46 & 346.89 \\
\hline Line.119 & 1 & 134.93 & 136.91 & 132.82 & 131.89 & 126.10 & 120.91 & 116.36 \\
\hline Line.119 & 2 & 134.93 & 136.91 & 132.82 & 131.89 & 126.10 & 120.91 & 116.36 \\
\hline Line. 120 & 1 & 267.49 & 271.43 & 263.33 & 261.48 & 249.99 & 239.70 & 230.68 \\
\hline Line. 120 & 2 & 267.49 & 271.43 & 263.33 & 261.48 & 249.99 & 239.70 & 230.68 \\
\hline Line. 121 & 1 & 0.01 & 0.01 & 0.01 & 0.01 & 0.01 & 0.01 & 0.01 \\
\hline Line.121 & 2 & 0.01 & 0.01 & 0.01 & 0.01 & 0.01 & 0.01 & 0.01 \\
\hline Line.122 & 1 & 267.52 & 271.46 & 263.36 & 261.51 & 250.01 & 239.73 & 230.70 \\
\hline Line.122 & 2 & 267.52 & 271.46 & 263.36 & 261.51 & 250.01 & 239.73 & 230.70 \\
\hline Line. 123 & 1 & 136.70 & 138.71 & 134.57 & 133.62 & 127.75 & 122.50 & 117.89 \\
\hline Line. 123 & 2 & 136.70 & 138.71 & 134.57 & 133.62 & 127.75 & 122.50 & 117.89 \\
\hline Line. 124 & 1 & 136.72 & 138.74 & 134.59 & 133.65 & 127.78 & 122.52 & 117.91 \\
\hline Line. 124 & 2 & 136.72 & 138.74 & 134.59 & 133.65 & 127.78 & 122.52 & 117.91 \\
\hline Line. 125 & 1 & 0.01 & 0.01 & 0.01 & 0.01 & 0.01 & 0.01 & 0.01 \\
\hline Line.125 & 2 & 0.01 & 0.01 & 0.01 & 0.01 & 0.01 & 0.01 & 0.01 \\
\hline Line.126 & 1 & 267.54 & 271.48 & 263.37 & 261.52 & 250.03 & 239.74 & 230.72 \\
\hline Line.126 & 2 & 267.54 & 271.48 & 263.37 & 261.52 & 250.03 & 239.74 & 230.72 \\
\hline Line.127 & 1 & 133.50 & 135.46 & 131.42 & 130.49 & 124.76 & 119.62 & 115.12 \\
\hline
\end{tabular}




\begin{tabular}{|c|c|c|c|c|c|c|c|c|}
\hline Element & Terminal & \multicolumn{7}{|c|}{ Faulty Node } \\
\hline & & $\mathbf{7 0 5}$ & $\mathbf{7 1 3}$ & $\mathbf{7 0 4}$ & $\mathbf{7 1 4}$ & $\mathbf{7 2 0}$ & $\mathbf{7 0 6}$ & $\mathbf{7 0 7}$ \\
\hline Line.127 & 2 & 133.50 & 135.46 & 131.42 & 130.49 & 124.76 & 119.62 & 115.12 \\
\hline Line.128 & 1 & 133.53 & 135.50 & 131.45 & 130.52 & 124.79 & 119.65 & 115.15 \\
\hline Line.128 & 2 & 133.53 & 135.50 & 131.45 & 130.52 & 124.79 & 119.65 & 115.15 \\
\hline Line.129 & 1 & 133.55 & 135.52 & 131.47 & 130.55 & 124.81 & 119.67 & 115.17 \\
\hline Line.129 & 2 & 133.55 & 135.52 & 131.47 & 130.55 & 124.81 & 119.67 & 115.17 \\
\hline Line.130 & 1 & 133.58 & 135.55 & 131.50 & 130.57 & 124.84 & 119.70 & 115.19 \\
\hline Line.130 & 2 & 133.58 & 135.55 & 131.50 & 130.57 & 124.84 & 119.70 & 115.19 \\
\hline Line.131 & 1 & 0.01 & 0.01 & 0.01 & 0.01 & 0.01 & 0.01 & 0.01 \\
\hline Line.131 & 2 & 0.01 & 0.01 & 0.01 & 0.01 & 0.01 & 0.01 & 0.01 \\
\hline Line.132 & 1 & 134.08 & 136.05 & 131.99 & 131.06 & 125.30 & 120.15 & 115.63 \\
\hline Line.132 & 2 & 134.08 & 136.05 & 131.99 & 131.06 & 125.30 & 120.15 & 115.63 \\
\hline Line.133 & 1 & 134.11 & 136.08 & 132.02 & 131.09 & 125.33 & 120.18 & 115.65 \\
\hline Line.133 & 2 & 134.11 & 136.08 & 132.02 & 131.09 & 125.33 & 120.18 & 115.65 \\
\hline Line.134 & 1 & 0.01 & 0.01 & 0.01 & 0.01 & 0.01 & 0.01 & 0.01 \\
\hline Line.134 & 2 & 0.01 & 0.01 & 0.01 & 0.01 & 0.01 & 0.01 & 0.01 \\
\hline
\end{tabular}

Table 4-16: $I_{2}$ value, zone 1, LL fault, $50 \%$ penetration

\begin{tabular}{|c|c|c|c|c|c|c|c|c|}
\hline Element & Terminal & \multicolumn{7}{|c|}{ Faulty Node } \\
\hline & & $\mathbf{7 0 5}$ & $\mathbf{7 1 3}$ & $\mathbf{7 0 4}$ & $\mathbf{7 1 4}$ & $\mathbf{7 2 0}$ & $\mathbf{7 0 6}$ & $\mathbf{7 0 7}$ \\
\hline Vsource & 1 & 7.54 & 7.66 & 7.45 & 7.39 & 7.12 & 6.87 & 6.59 \\
\hline Vsource & 2 & 7.54 & 7.66 & 7.45 & 7.39 & 7.12 & 6.87 & 6.59 \\
\hline Subxf & 1 & 7.54 & 7.66 & 7.45 & 7.39 & 7.12 & 6.87 & 6.59 \\
\hline Subxf & 2 & 361.13 & 366.95 & 356.86 & 354.34 & 341.25 & 329.40 & 315.65 \\
\hline Xfm1 & 1 & 0.00 & 0.00 & 0.00 & 0.00 & 0.00 & 0.00 & 0.00 \\
\hline Xfm1 & 2 & 0.00 & 0.00 & 0.00 & 0.00 & 0.00 & 0.00 & 0.00 \\
\hline Line.11 & 1 & 328.58 & 333.85 & 324.67 & 322.39 & 310.47 & 299.68 & 287.23 \\
\hline Line.11 & 2 & 328.56 & 333.82 & 324.65 & 322.36 & 310.45 & 299.66 & 287.20 \\
\hline Line.12 & 1 & 556.48 & 44.62 & 43.67 & 43.45 & 42.19 & 41.07 & 39.94 \\
\hline Line.12 & 2 & 556.48 & 44.63 & 43.67 & 43.46 & 42.20 & 41.07 & 39.94 \\
\hline Line.13 & 1 & 95.27 & 514.12 & 499.64 & 496.04 & 477.14 & 459.96 & 440.17 \\
\hline Line.13 & 2 & 95.27 & 514.12 & 499.64 & 496.04 & 477.13 & 459.96 & 440.17 \\
\hline Line.14 & 1 & 133.36 & 135.80 & 131.42 & 130.33 & 124.59 & 119.32 & 113.18 \\
\hline Line.14 & 2 & 133.39 & 135.84 & 131.46 & 130.36 & 124.62 & 119.36 & 113.22 \\
\hline Line.15 & 1 & 95.60 & 98.04 & 498.78 & 495.21 & 476.33 & 459.20 & 439.73 \\
\hline Line.15 & 2 & 95.61 & 98.05 & 498.77 & 495.20 & 476.32 & 459.20 & 439.73 \\
\hline Line.16 & 1 & 60.12 & 60.79 & 60.88 & 60.75 & 512.63 & 493.94 & 472.39 \\
\hline Line.16 & 2 & 60.13 & 60.81 & 60.89 & 60.77 & 512.62 & 493.93 & 472.38 \\
\hline
\end{tabular}




\begin{tabular}{|c|c|c|c|c|c|c|c|c|}
\hline \multirow[t]{2}{*}{ Element } & \multirow[t]{2}{*}{ Terminal } & \multicolumn{7}{|c|}{ Faulty Node } \\
\hline & & 705 & 713 & 704 & 714 & 720 & 706 & 707 \\
\hline Line.17 & 1 & 4.18 & 4.26 & 4.29 & 4.28 & 4.34 & 549.64 & 4.24 \\
\hline Line.17 & 2 & 4.18 & 4.27 & 4.30 & 4.29 & 4.35 & 549.63 & 4.25 \\
\hline Line.18 & 1 & 4.18 & 4.27 & 4.30 & 4.29 & 4.35 & 4.37 & 4.25 \\
\hline Line.18 & 2 & 4.19 & 4.27 & 4.30 & 4.29 & 4.35 & 4.38 & 4.25 \\
\hline Line.19 & 1 & 56.12 & 56.91 & 57.03 & 56.88 & 57.16 & 56.26 & 476.27 \\
\hline Line.19 & 2 & 56.13 & 56.93 & 57.04 & 56.89 & 57.17 & 56.27 & 476.26 \\
\hline Line.110 & 1 & 17.96 & 18.07 & 18.08 & 18.06 & 18.09 & 18.00 & 18.10 \\
\hline Line.110 & 2 & 17.96 & 18.07 & 18.08 & 18.06 & 18.09 & 18.00 & 18.10 \\
\hline Line.111 & 1 & 42.75 & 44.23 & 44.46 & 44.19 & 44.72 & 43.37 & 45.13 \\
\hline Line.111 & 2 & 42.76 & 44.24 & 44.47 & 44.20 & 44.73 & 43.38 & 45.14 \\
\hline Line.112 & 1 & 38.64 & 40.51 & 40.81 & 555.29 & 38.93 & 37.47 & 35.52 \\
\hline Line.112 & 2 & 38.64 & 40.51 & 40.82 & 555.29 & 38.93 & 37.47 & 35.52 \\
\hline Line.113 & 1 & 38.78 & 40.83 & 41.15 & 41.18 & 39.00 & 37.38 & 35.31 \\
\hline Line.113 & 2 & 38.78 & 40.84 & 41.16 & 41.19 & 39.01 & 37.39 & 35.31 \\
\hline Line.114 & 1 & 9.84 & 9.93 & 10.04 & 10.08 & 10.20 & 10.32 & 10.54 \\
\hline Line.114 & 2 & 9.84 & 9.93 & 10.04 & 10.08 & 10.20 & 10.32 & 10.54 \\
\hline Line.115 & 1 & 47.39 & 46.59 & 45.60 & 45.36 & 44.08 & 42.92 & 41.58 \\
\hline Line.115 & 2 & 47.39 & 46.59 & 45.61 & 45.36 & 44.08 & 42.92 & 41.58 \\
\hline Line.116 & 1 & 36.21 & 36.99 & 35.75 & 35.43 & 33.83 & 32.35 & 30.51 \\
\hline Line.116 & 2 & 36.22 & 36.99 & 35.76 & 35.43 & 33.83 & 32.36 & 30.51 \\
\hline Line.117 & 1 & 97.41 & 99.07 & 95.92 & 95.15 & 91.01 & 87.23 & 82.98 \\
\hline Line.117 & 2 & 97.42 & 99.08 & 95.93 & 95.16 & 91.02 & 87.24 & 82.99 \\
\hline Line.118 & 1 & 98.13 & 99.97 & 96.86 & 96.05 & 91.95 & 88.14 & 83.47 \\
\hline Line.118 & 2 & 98.13 & 99.98 & 96.86 & 96.06 & 91.96 & 88.14 & 83.48 \\
\hline Line.119 & 1 & 47.78 & 48.34 & 47.34 & 47.10 & 45.79 & 44.60 & 43.24 \\
\hline Line.119 & 2 & 47.79 & 48.35 & 47.35 & 47.11 & 45.80 & 44.61 & 43.25 \\
\hline Line. 120 & 1 & 51.71 & 52.86 & 50.62 & 50.08 & 47.18 & 44.54 & 41.57 \\
\hline Line.120 & 2 & 51.71 & 52.86 & 50.62 & 50.08 & 47.18 & 44.54 & 41.57 \\
\hline Line.121 & 1 & 4.96 & 4.90 & 4.96 & 4.98 & 5.04 & 5.09 & 5.20 \\
\hline Line.121 & 2 & 4.96 & 4.90 & 4.96 & 4.97 & 5.03 & 5.09 & 5.20 \\
\hline Line. 122 & 1 & 51.79 & 53.08 & 50.90 & 50.33 & 47.49 & 44.84 & 41.53 \\
\hline Line.122 & 2 & 51.79 & 53.09 & 50.91 & 50.34 & 47.50 & 44.85 & 41.53 \\
\hline Line. 123 & 1 & 37.34 & 38.17 & 36.98 & 36.65 & 35.10 & 33.66 & 31.70 \\
\hline Line.123 & 2 & 37.34 & 38.18 & 36.99 & 36.65 & 35.11 & 33.66 & 31.70 \\
\hline Line. 124 & 1 & 37.95 & 38.64 & 37.65 & 37.37 & 36.04 & 34.77 & 33.09 \\
\hline Line.124 & 2 & 37.96 & 38.64 & 37.65 & 37.37 & 36.04 & 34.78 & 33.09 \\
\hline Line.125 & 1 & 7.21 & 7.32 & 7.12 & 7.07 & 6.81 & 6.58 & 6.30 \\
\hline Line. 125 & 2 & 7.21 & 7.32 & 7.12 & 7.07 & 6.81 & 6.58 & 6.30 \\
\hline Line.126 & 1 & 61.96 & 63.24 & 60.93 & 60.36 & 57.34 & 54.59 & 51.42 \\
\hline
\end{tabular}




\begin{tabular}{|c|c|c|c|c|c|c|c|c|}
\hline Element & Terminal & \multicolumn{7}{|c|}{ Faulty Node } \\
\hline & & $\mathbf{7 0 5}$ & $\mathbf{7 1 3}$ & $\mathbf{7 0 4}$ & $\mathbf{7 1 4}$ & $\mathbf{7 2 0}$ & $\mathbf{7 0 6}$ & $\mathbf{7 0 7}$ \\
\hline Line.126 & 2 & 61.97 & 63.25 & 60.94 & 60.37 & 57.35 & 54.60 & 51.43 \\
\hline Line.127 & 1 & 19.32 & 20.22 & 19.02 & 18.66 & 17.13 & 15.66 & 13.42 \\
\hline Line.127 & 2 & 19.34 & 20.24 & 19.03 & 18.67 & 17.14 & 15.67 & 13.43 \\
\hline Line.128 & 1 & 35.98 & 36.84 & 35.37 & 35.01 & 33.11 & 31.40 & 29.44 \\
\hline Line.128 & 2 & 35.98 & 36.84 & 35.38 & 35.01 & 33.12 & 31.41 & 29.44 \\
\hline Line.129 & 1 & 38.98 & 39.41 & 38.39 & 38.17 & 36.81 & 35.62 & 34.52 \\
\hline Line.129 & 2 & 38.98 & 39.41 & 38.40 & 38.17 & 36.82 & 35.62 & 34.53 \\
\hline Line.130 & 1 & 40.72 & 41.34 & 40.20 & 39.92 & 38.43 & 37.08 & 35.58 \\
\hline Line.130 & 2 & 40.72 & 41.34 & 40.21 & 39.93 & 38.44 & 37.09 & 35.59 \\
\hline Line.131 & 1 & 10.04 & 9.92 & 10.03 & 10.07 & 10.19 & 10.30 & 10.51 \\
\hline Line.131 & 2 & 10.04 & 9.92 & 10.03 & 10.07 & 10.19 & 10.30 & 10.51 \\
\hline Line.132 & 1 & 43.08 & 43.58 & 42.54 & 42.30 & 40.93 & 39.70 & 38.45 \\
\hline Line.132 & 2 & 43.09 & 43.58 & 42.55 & 42.31 & 40.94 & 39.71 & 38.46 \\
\hline Line.133 & 1 & 45.37 & 46.00 & 44.91 & 44.63 & 43.21 & 41.91 & 40.40 \\
\hline Line.133 & 2 & 45.39 & 46.02 & 44.92 & 44.65 & 43.22 & 41.93 & 40.42 \\
\hline Line.134 & 1 & 10.05 & 9.93 & 10.04 & 10.08 & 10.20 & 10.31 & 10.52 \\
\hline Line.134 & 2 & 10.05 & 9.93 & 10.04 & 10.08 & 10.20 & 10.31 & 10.52 \\
\hline Line.135 & 1 & 328.30 & 333.59 & 324.42 & 322.13 & 310.23 & 299.45 & 286.95 \\
\hline
\end{tabular}

Table 4-17: $I_{1}$ value, zone 2,3 phase fault, $50 \%$ penetration

\begin{tabular}{|c|c|c|c|c|c|c|}
\hline Element & Terminal & \multicolumn{5}{|c|}{ Faulty Node } \\
\hline & & $\mathbf{7 4 4}$ & $\mathbf{7 2 7}$ & $\mathbf{7 3 0}$ & $\mathbf{7 0 9}$ & $\mathbf{7 0 8}$ \\
\hline Vsource & 1 & 16.61 & 16.82 & 16.67 & 16.53 & 16.29 \\
\hline Vsource & 2 & 16.61 & 16.82 & 16.67 & 16.53 & 16.29 \\
\hline Subxf & 1 & 16.61 & 16.82 & 16.67 & 16.53 & 16.29 \\
\hline Subxf & 2 & 795.68 & 806.11 & 798.83 & 792.08 & 780.76 \\
\hline Xfm1 & 1 & 0.00 & 0.00 & 0.00 & 0.00 & 0.00 \\
\hline Xfm1 & 2 & 0.00 & 0.00 & 0.00 & 0.00 & 0.00 \\
\hline Line.11 & 1 & 721.05 & 730.63 & 723.98 & 717.78 & 707.37 \\
\hline Line.11 & 2 & 721.05 & 730.63 & 723.98 & 717.78 & 707.38 \\
\hline Line.12 & 1 & 53.26 & 54.23 & 53.61 & 52.98 & 51.92 \\
\hline Line.12 & 2 & 53.26 & 54.23 & 53.61 & 52.98 & 51.92 \\
\hline Line.13 & 1 & 90.40 & 92.21 & 91.16 & 89.98 & 88.00 \\
\hline Line.13 & 2 & 90.40 & 92.21 & 91.16 & 89.98 & 88.00 \\
\hline Line.14 & 1 & 863.39 & 875.84 & 867.39 & 859.34 & 845.83 \\
\hline Line.14 & 2 & 863.40 & 875.84 & 867.40 & 859.34 & 845.83 \\
\hline Line.15 & 1 & 93.26 & 94.96 & 93.91 & 92.81 & 90.96 \\
\hline Line.15 & 2 & 93.26 & 94.96 & 93.91 & 92.81 & 90.96 \\
\hline
\end{tabular}




\begin{tabular}{|l|c|c|c|c|c|c|}
\hline Element & Terminal & \multicolumn{5}{|c|}{ Faulty Node } \\
\hline & & $\mathbf{7 4 4}$ & $\mathbf{7 2 7}$ & $\mathbf{7 3 0}$ & $\mathbf{7 0 9}$ & $\mathbf{7 0 8}$ \\
\hline Line.16 & 1 & 41.93 & 42.76 & 42.34 & 41.80 & 40.90 \\
\hline Line.16 & 2 & 41.93 & 42.75 & 42.34 & 41.80 & 40.89 \\
\hline Line.17 & 1 & 1.48 & 1.41 & 1.43 & 1.48 & 1.56 \\
\hline Line.17 & 2 & 1.48 & 1.41 & 1.43 & 1.48 & 1.56 \\
\hline Line.18 & 1 & 1.48 & 1.41 & 1.43 & 1.48 & 1.56 \\
\hline Line.18 & 2 & 1.48 & 1.41 & 1.43 & 1.48 & 1.56 \\
\hline Line.19 & 1 & 46.15 & 46.82 & 46.40 & 45.96 & 45.22 \\
\hline Line.19 & 2 & 46.14 & 46.82 & 46.40 & 45.96 & 45.22 \\
\hline Line.110 & 1 & 19.36 & 19.36 & 19.36 & 19.36 & 19.36 \\
\hline Line.110 & 2 & 19.36 & 19.36 & 19.36 & 19.36 & 19.36 \\
\hline Line.111 & 1 & 64.73 & 65.53 & 64.95 & 64.43 & 63.56 \\
\hline Line.111 & 2 & 64.73 & 65.53 & 64.95 & 64.43 & 63.55 \\
\hline Line.112 & 1 & 51.66 & 52.48 & 51.90 & 51.36 & 50.47 \\
\hline Line.112 & 2 & 51.66 & 52.48 & 51.89 & 51.36 & 50.47 \\
\hline Line.113 & 1 & 56.14 & 56.98 & 56.37 & 55.83 & 54.92 \\
\hline Line.113 & 2 & 56.14 & 56.98 & 56.37 & 55.83 & 54.92 \\
\hline Line.114 & 1 & 3.00 & 2.85 & 2.90 & 3.00 & 3.16 \\
\hline Line.114 & 2 & 3.00 & 2.85 & 2.90 & 3.00 & 3.16 \\
\hline Line.115 & 1 & 56.19 & 57.03 & 56.44 & 55.89 & 54.97 \\
\hline Line.115 & 2 & 56.19 & 57.03 & 56.44 & 55.89 & 54.97 \\
\hline Line.116 & 1 & 1005.21 & 1020.82 & 60.29 & 59.56 & 58.35 \\
\hline Line.116 & 2 & 1005.21 & 1020.82 & 60.29 & 59.56 & 58.35 \\
\hline Line.117 & 1 & 142.35 & 145.45 & 926.97 & 918.17 & 903.41 \\
\hline Line.117 & 2 & 142.35 & 145.45 & 926.97 & 918.17 & 903.41 \\
\hline Line.118 & 1 & 144.77 & 147.75 & 152.78 & 916.11 & 901.21 \\
\hline Line.118 & 2 & 144.77 & 147.75 & 152.78 & 916.11 & 901.21 \\
\hline Line.119 & 1 & 61.31 & 62.20 & 63.51 & 63.56 & 62.54 \\
\hline Line.119 & 2 & 61.30 & 62.20 & 63.51 & 63.56 & 62.53 \\
\hline Line.120 & 1 & 83.49 & 85.56 & 89.28 & 89.54 & 963.46 \\
\hline Line.120 & 2 & 83.49 & 85.56 & 89.28 & 89.54 & 963.46 \\
\hline Line.121 & 1 & 1.36 & 1.29 & 1.10 & 1.08 & 1.05 \\
\hline Line.121 & 2 & 1.36 & 1.29 & 1.10 & 1.08 & 1.05 \\
\hline Line.122 & 1 & 84.83 & 86.84 & 90.38 & 90.62 & 90.87 \\
\hline Line.122 & 2 & 84.83 & 86.84 & 90.38 & 90.62 & 90.87 \\
\hline Line.123 & 1 & 1004.05 & 63.67 & 61.53 & 60.84 & 59.69 \\
\hline Line.123 & 2 & 1004.05 & 63.67 & 61.52 & 60.84 & 59.69 \\
\hline Line.124 & 1 & 68.00 & 68.01 & 66.54 & 66.02 & 65.16 \\
\hline Line.124 & 2 & 68.00 & 68.01 & 66.54 & 66.02 & 65.15 \\
\hline Line.125 & 1 & 3.23 & 3.30 & 3.84 & 3.98 & 4.23 \\
\hline
\end{tabular}




\begin{tabular}{|l|c|c|c|c|c|c|}
\hline Element & Terminal & \multicolumn{5}{|c|}{ Faulty Node } \\
\hline & & $\mathbf{7 4 4}$ & $\mathbf{7 2 7}$ & $\mathbf{7 3 0}$ & $\mathbf{7 0 9}$ & $\mathbf{7 0 8}$ \\
\hline Line.125 & 2 & 3.23 & 3.30 & 3.84 & 3.98 & 4.23 \\
\hline Line.126 & 1 & 94.92 & 96.98 & 100.54 & 100.77 & 101.00 \\
\hline Line.126 & 2 & 94.91 & 96.98 & 100.54 & 100.77 & 101.00 \\
\hline Line.127 & 1 & 35.70 & 36.69 & 38.53 & 38.67 & 38.82 \\
\hline Line.127 & 2 & 35.70 & 36.69 & 38.53 & 38.67 & 38.82 \\
\hline Line.128 & 1 & 52.26 & 53.36 & 55.23 & 55.36 & 55.47 \\
\hline Line.128 & 2 & 52.26 & 53.36 & 55.23 & 55.35 & 55.47 \\
\hline Line.129 & 1 & 56.39 & 57.28 & 58.62 & 58.69 & 58.73 \\
\hline Line.129 & 2 & 56.39 & 57.28 & 58.62 & 58.69 & 58.73 \\
\hline Line.130 & 1 & 59.23 & 59.99 & 60.96 & 60.98 & 60.97 \\
\hline Line.130 & 2 & 59.23 & 59.99 & 60.96 & 60.98 & 60.97 \\
\hline Line.131 & 1 & 2.87 & 2.72 & 2.35 & 2.30 & 2.25 \\
\hline Line.131 & 2 & 2.87 & 2.72 & 2.35 & 2.30 & 2.25 \\
\hline Line.132 & 1 & 60.70 & 61.67 & 63.19 & 63.27 & 63.33 \\
\hline Line.132 & 2 & 60.70 & 61.67 & 63.19 & 63.27 & 63.33 \\
\hline Line.133 & 1 & 63.54 & 64.38 & 65.52 & 65.56 & 65.56 \\
\hline Line.133 & 2 & 63.54 & 64.38 & 65.52 & 65.56 & 65.56 \\
\hline Line.134 & 1 & 2.86 & 2.71 & 2.34 & 2.29 & 2.24 \\
\hline Line.134 & 2 & 2.86 & 2.71 & 2.34 & 2.29 & 2.24 \\
\hline
\end{tabular}

Table 4-18: $I_{r}$ value, zone 2, SLG fault, $50 \%$ penetration

\begin{tabular}{|c|c|c|c|c|c|c|}
\hline Element & Terminal & \multicolumn{6}{|c|}{ Faulty Node } \\
\hline & & $\mathbf{7 4 4}$ & $\mathbf{7 2 7}$ & $\mathbf{7 3 0}$ & $\mathbf{7 0 9}$ & $\mathbf{7 0 8}$ \\
\hline Vsource & 1 & 0.00 & 0.00 & 0.00 & 0.00 & 0.00 \\
\hline Vsource & 2 & 0.00 & 0.00 & 0.00 & 0.00 & 0.00 \\
\hline Subxf & 1 & 0.00 & 0.00 & 0.00 & 0.00 & 0.00 \\
\hline Subxf & 2 & 0.00 & 0.00 & 0.00 & 0.00 & 0.00 \\
\hline Xfm1 & 1 & 0.00 & 0.00 & 0.00 & 0.00 & 0.00 \\
\hline Xfm1 & 2 & 0.00 & 0.00 & 0.00 & 0.00 & 0.00 \\
\hline Line.11 & 1 & 0.21 & 0.22 & 0.21 & 0.21 & 0.21 \\
\hline Line.11 & 2 & 0.21 & 0.22 & 0.21 & 0.21 & 0.21 \\
\hline Line.12 & 1 & 127.96 & 130.28 & 129.08 & 127.80 & 125.48 \\
\hline Line.12 & 2 & 127.96 & 130.28 & 129.08 & 127.80 & 125.48 \\
\hline Line.13 & 1 & 251.88 & 256.44 & 254.08 & 251.56 & 246.99 \\
\hline Line.13 & 2 & 251.88 & 256.44 & 254.08 & 251.56 & 246.99 \\
\hline Line.14 & 1 & 379.47 & 386.35 & 382.79 & 379.00 & 372.11 \\
\hline Line.14 & 2 & 379.47 & 386.35 & 382.79 & 379.00 & 372.11 \\
\hline
\end{tabular}




\begin{tabular}{|c|c|c|c|c|c|c|}
\hline \multirow[t]{2}{*}{ Element } & \multirow[t]{2}{*}{ Terminal } & \multicolumn{5}{|c|}{$\begin{array}{l}\text { Faulty Node } \\
\end{array}$} \\
\hline & & 744 & 727 & 730 & 709 & 708 \\
\hline Line.15 & 1 & 251.89 & 256.46 & 254.10 & 251.58 & 247.01 \\
\hline Line. 15 & 2 & 251.89 & 256.46 & 254.10 & 251.58 & 247.01 \\
\hline Line.16 & 1 & 125.45 & 127.73 & 126.55 & 125.30 & 123.02 \\
\hline Line. 16 & 2 & 125.45 & 127.73 & 126.55 & 125.30 & 123.02 \\
\hline Line.17 & 1 & 0.04 & 0.04 & 0.04 & 0.04 & 0.04 \\
\hline Line.17 & 2 & 0.04 & 0.04 & 0.04 & 0.04 & 0.04 \\
\hline Line.18 & 1 & 0.01 & 0.01 & 0.01 & 0.01 & 0.01 \\
\hline Line.18 & 2 & 0.01 & 0.01 & 0.01 & 0.01 & 0.01 \\
\hline Line.19 & 1 & 125.54 & 127.81 & 126.64 & 125.38 & 123.10 \\
\hline Line.19 & 2 & 125.54 & 127.81 & 126.64 & 125.38 & 123.10 \\
\hline Line. 110 & 1 & 0.01 & 0.01 & 0.01 & 0.01 & 0.00 \\
\hline Line. 110 & 2 & 0.01 & 0.01 & 0.01 & 0.01 & 0.00 \\
\hline Line.111 & 1 & 125.58 & 127.86 & 126.68 & 125.43 & 123.15 \\
\hline Line.111 & 2 & 125.58 & 127.86 & 126.68 & 125.43 & 123.15 \\
\hline Line.112 & 1 & 126.50 & 128.80 & 127.61 & 126.35 & 124.05 \\
\hline Line.112 & 2 & 126.50 & 128.80 & 127.61 & 126.35 & 124.05 \\
\hline Line.113 & 1 & 126.51 & 128.80 & 127.62 & 126.35 & 124.05 \\
\hline Line.113 & 2 & 126.51 & 128.80 & 127.62 & 126.35 & 124.05 \\
\hline Line.114 & 1 & 0.01 & 0.01 & 0.01 & 0.01 & 0.01 \\
\hline Line.114 & 2 & 0.01 & 0.01 & 0.01 & 0.01 & 0.01 \\
\hline Line.115 & 1 & 127.99 & 130.31 & 129.11 & 127.83 & 125.51 \\
\hline Line.115 & 2 & 127.99 & 130.31 & 129.11 & 127.83 & 125.51 \\
\hline Line.116 & 1 & 778.21 & 792.33 & 136.76 & 135.40 & 132.94 \\
\hline Line.116 & 2 & 778.21 & 792.33 & 136.76 & 135.40 & 132.94 \\
\hline Line.117 & 1 & 398.93 & 406.16 & 519.33 & 514.19 & 504.84 \\
\hline Line.117 & 2 & 398.93 & 406.16 & 519.33 & 514.19 & 504.84 \\
\hline Line.118 & 1 & 398.96 & 406.19 & 415.54 & 514.16 & 504.81 \\
\hline Line.118 & 2 & 398.96 & 406.19 & 415.54 & 514.16 & 504.81 \\
\hline Line.119 & 1 & 133.82 & 136.25 & 139.38 & 139.49 & 136.95 \\
\hline Line.119 & 2 & 133.82 & 136.25 & 139.38 & 139.49 & 136.95 \\
\hline Line. 120 & 1 & 265.31 & 270.12 & 276.34 & 276.55 & 641.40 \\
\hline Line.120 & 2 & 265.31 & 270.12 & 276.34 & 276.55 & 641.40 \\
\hline Line.121 & 1 & 0.01 & 0.01 & 0.01 & 0.01 & 0.01 \\
\hline Line.121 & 2 & 0.01 & 0.01 & 0.01 & 0.01 & 0.01 \\
\hline Line.122 & 1 & 265.34 & 270.15 & 276.37 & 276.58 & 276.29 \\
\hline Line.122 & 2 & 265.34 & 270.15 & 276.37 & 276.58 & 276.29 \\
\hline Line.123 & 1 & 778.20 & 140.27 & 136.77 & 135.41 & 132.95 \\
\hline Line.123 & 2 & 778.20 & 140.27 & 136.77 & 135.41 & 132.95 \\
\hline Line.124 & 1 & 139.94 & 140.30 & 136.79 & 135.44 & 132.98 \\
\hline
\end{tabular}




\begin{tabular}{|l|c|c|c|c|c|c|}
\hline Element & Terminal & \multicolumn{7}{|c|}{ Faulty Node } \\
\hline & & $\mathbf{7 4 4}$ & $\mathbf{7 2 7}$ & $\mathbf{7 3 0}$ & $\mathbf{7 0 9}$ & $\mathbf{7 0 8}$ \\
\hline Line.124 & 2 & 139.94 & 140.30 & 136.79 & 135.44 & 132.98 \\
\hline Line.125 & 1 & 0.01 & 0.01 & 0.01 & 0.01 & 0.01 \\
\hline Line.125 & 2 & 0.01 & 0.01 & 0.01 & 0.01 & 0.01 \\
\hline Line.126 & 1 & 265.35 & 270.17 & 276.38 & 276.60 & 276.31 \\
\hline Line.126 & 2 & 265.35 & 270.17 & 276.38 & 276.60 & 276.31 \\
\hline Line.127 & 1 & 132.40 & 134.81 & 137.91 & 138.02 & 137.87 \\
\hline Line.127 & 2 & 132.40 & 134.81 & 137.91 & 138.02 & 137.87 \\
\hline Line.128 & 1 & 132.44 & 134.84 & 137.94 & 138.05 & 137.91 \\
\hline Line.128 & 2 & 132.44 & 134.84 & 137.94 & 138.05 & 137.91 \\
\hline Line.129 & 1 & 132.46 & 134.86 & 137.97 & 138.07 & 137.93 \\
\hline Line.129 & 2 & 132.46 & 134.86 & 137.97 & 138.07 & 137.93 \\
\hline Line.130 & 1 & 132.49 & 134.89 & 138.00 & 138.10 & 137.96 \\
\hline Line.130 & 2 & 132.49 & 134.89 & 138.00 & 138.10 & 137.96 \\
\hline Line.131 & 1 & 0.01 & 0.01 & 0.01 & 0.01 & 0.01 \\
\hline Line.131 & 2 & 0.01 & 0.01 & 0.01 & 0.01 & 0.01 \\
\hline Line.132 & 1 & 132.98 & 135.39 & 138.51 & 138.62 & 138.47 \\
\hline Line.132 & 2 & 132.98 & 135.39 & 138.51 & 138.62 & 138.47 \\
\hline Line.133 & 1 & 133.01 & 135.43 & 138.54 & 138.65 & 138.50 \\
\hline Line.133 & 2 & 133.01 & 135.43 & 138.54 & 138.65 & 138.50 \\
\hline Line.134 & 1 & 0.01 & 0.01 & 0.01 & 0.01 & 0.01 \\
\hline Line.134 & 2 & 0.01 & 0.01 & 0.01 & 0.01 & 0.01 \\
\hline
\end{tabular}


Table 4-19: $I_{2}$ value, zone 2, LL fault, $50 \%$ penetration

\begin{tabular}{|c|c|c|c|c|c|c|}
\hline Element & Terminal & \multicolumn{5}{|c|}{ Faulty Node } \\
\hline & & $\mathbf{7 4 4}$ & $\mathbf{7 2 7}$ & $\mathbf{7 3 0}$ & $\mathbf{7 0 9}$ & $\mathbf{7 0 8}$ \\
\hline Vsource & 1 & 7.23 & 7.35 & 7.26 & 7.18 & 7.05 \\
\hline Vsource & 2 & 7.23 & 7.35 & 7.26 & 7.18 & 7.05 \\
\hline Subxf & 1 & 7.23 & 7.35 & 7.26 & 7.18 & 7.05 \\
\hline Subxf & 2 & 346.64 & 352.28 & 347.97 & 344.15 & 337.73 \\
\hline Xfm1 & 1 & 0.00 & 0.00 & 0.00 & 0.00 & 0.00 \\
\hline Xfm1 & 2 & 0.00 & 0.00 & 0.00 & 0.00 & 0.00 \\
\hline Line.11 & 1 & 315.36 & 320.48 & 316.55 & 313.08 & 307.24 \\
\hline Line.11 & 2 & 315.33 & 320.46 & 316.52 & 313.06 & 307.21 \\
\hline Line.12 & 1 & 42.62 & 43.16 & 42.68 & 42.32 & 41.71 \\
\hline Line.12 & 2 & 42.63 & 43.16 & 42.68 & 42.32 & 41.71 \\
\hline Line.13 & 1 & 92.18 & 93.21 & 92.27 & 91.57 & 90.38 \\
\hline Line.13 & 2 & 92.19 & 93.22 & 92.28 & 91.58 & 90.39 \\
\hline Line.14 & 1 & 449.49 & 456.17 & 450.81 & 446.29 & 438.67 \\
\hline Line.14 & 2 & 449.45 & 456.13 & 450.78 & 446.26 & 438.64 \\
\hline Line.15 & 1 & 92.65 & 93.69 & 92.80 & 92.09 & 90.89 \\
\hline Line.15 & 2 & 92.66 & 93.70 & 92.81 & 92.10 & 90.89 \\
\hline Line.16 & 1 & 58.80 & 59.18 & 58.76 & 58.50 & 58.05 \\
\hline Line.16 & 2 & 58.81 & 59.19 & 58.77 & 58.51 & 58.06 \\
\hline Line.17 & 1 & 4.19 & 4.20 & 4.20 & 4.19 & 4.18 \\
\hline Line.17 & 2 & 4.19 & 4.20 & 4.21 & 4.20 & 4.18 \\
\hline Line.18 & 1 & 4.19 & 4.20 & 4.21 & 4.20 & 4.18 \\
\hline Line.18 & 2 & 4.19 & 4.21 & 4.21 & 4.20 & 4.19 \\
\hline Line.19 & 1 & 54.86 & 55.26 & 54.86 & 54.59 & 54.13 \\
\hline Line.19 & 2 & 54.87 & 55.27 & 54.87 & 54.60 & 54.14 \\
\hline Line.110 & 1 & 17.87 & 17.91 & 17.88 & 17.85 & 17.80 \\
\hline Line.110 & 2 & 17.87 & 17.91 & 17.88 & 17.85 & 17.80 \\
\hline Line.111 & 1 & 41.22 & 41.80 & 41.34 & 40.94 & 40.28 \\
\hline Line.111 & 2 & 41.23 & 41.81 & 41.35 & 40.95 & 40.29 \\
\hline Line.112 & 1 & 37.14 & 37.81 & 37.38 & 36.92 & 36.15 \\
\hline Line.112 & 2 & 37.14 & 37.81 & 37.38 & 36.92 & 36.15 \\
\hline Line.113 & 1 & 37.08 & 37.82 & 37.32 & 36.82 & 35.98 \\
\hline Line.113 & 2 & 37.08 & 37.82 & 37.33 & 36.83 & 35.99 \\
\hline Line.114 & 1 & 10.09 & 10.03 & 10.03 & 10.06 & 10.12 \\
\hline Line.114 & 2 & 10.09 & 10.03 & 10.03 & 10.06 & 10.12 \\
\hline Line.115 & 1 & 44.57 & 45.12 & 44.67 & 44.30 & 43.66 \\
\hline Line.115 & 2 & 44.57 & 45.13 & 44.68 & 44.30 & 43.67 \\
\hline Line.116 & 1 & 547.34 & 555.88 & 36.70 & 36.22 & 35.40 \\
\hline
\end{tabular}




\begin{tabular}{|c|c|c|c|c|c|c|}
\hline Element & Terminal & & & ulty No & & \\
\hline & & 744 & 727 & 730 & 709 & 708 \\
\hline Line.116 & 2 & 547.34 & 555.88 & 36.71 & 36.22 & 35.40 \\
\hline Line.117 & 1 & 97.91 & 99.79 & 487.29 & 482.27 & 473.81 \\
\hline Line.117 & 2 & 97.92 & 99.80 & 487.28 & 482.26 & 473.80 \\
\hline Line.118 & 1 & 98.77 & 100.60 & 103.65 & 481.78 & 473.35 \\
\hline Line.118 & 2 & 98.77 & 100.60 & 103.65 & 481.78 & 473.34 \\
\hline Line.119 & 1 & 47.97 & 48.55 & 49.52 & 49.59 & 48.88 \\
\hline Line.119 & 2 & 47.98 & 48.56 & 49.53 & 49.60 & 48.89 \\
\hline Line. 120 & 1 & 52.04 & 53.40 & 55.47 & 55.60 & 520.45 \\
\hline Line. 120 & 2 & 52.04 & 53.40 & 55.48 & 55.60 & 520.44 \\
\hline Line. 121 & 1 & 4.93 & 4.90 & 4.83 & 4.83 & 4.82 \\
\hline Line.121 & 2 & 4.93 & 4.90 & 4.83 & 4.82 & 4.82 \\
\hline Line. 122 & 1 & 52.24 & 53.54 & 55.69 & 55.83 & 55.96 \\
\hline Line. 122 & 2 & 52.24 & 53.54 & 55.69 & 55.84 & 55.97 \\
\hline Line. 123 & 1 & 547.11 & 39.48 & 37.91 & 37.44 & 36.65 \\
\hline Line. 123 & 2 & 547.10 & 39.48 & 37.92 & 37.45 & 36.65 \\
\hline Line. 124 & 1 & 39.81 & 39.70 & 38.42 & 38.02 & 37.35 \\
\hline Line. 124 & 2 & 39.82 & 39.71 & 38.42 & 38.03 & 37.35 \\
\hline Line. 125 & 1 & 7.55 & 7.53 & 7.27 & 7.19 & 7.06 \\
\hline Line. 125 & 2 & 7.55 & 7.53 & 7.27 & 7.19 & 7.06 \\
\hline Line. 126 & 1 & 62.37 & 63.74 & 65.91 & 66.05 & 66.17 \\
\hline Line.126 & 2 & 62.38 & 63.75 & 65.92 & 66.06 & 66.18 \\
\hline Line. 127 & 1 & 19.70 & 20.41 & 21.76 & 21.86 & 21.97 \\
\hline Line. 127 & 2 & 19.71 & 20.43 & 21.77 & 21.87 & 21.98 \\
\hline Line. 128 & 1 & 36.28 & 37.13 & 38.54 & 38.64 & 38.72 \\
\hline Line. 128 & 2 & 36.28 & 37.14 & 38.55 & 38.64 & 38.73 \\
\hline Line.129 & 1 & 39.07 & 39.67 & 40.51 & 40.55 & 40.58 \\
\hline Line.129 & 2 & 39.07 & 39.68 & 40.51 & 40.56 & 40.59 \\
\hline Line.130 & 1 & 40.92 & 41.58 & 42.64 & 42.71 & 42.78 \\
\hline Line.130 & 2 & 40.92 & 41.59 & 42.65 & 42.72 & 42.78 \\
\hline Line.131 & 1 & 9.98 & 9.92 & 9.77 & 9.77 & 9.76 \\
\hline Line.131 & 2 & 9.98 & 9.92 & 9.77 & 9.77 & 9.76 \\
\hline Line.132 & 1 & 43.21 & 43.82 & 44.73 & 44.79 & 44.84 \\
\hline Line.132 & 2 & 43.22 & 43.83 & 44.74 & 44.80 & 44.85 \\
\hline Line.133 & 1 & 45.59 & 46.22 & 47.29 & 47.36 & 47.44 \\
\hline Line.133 & 2 & 45.60 & 46.24 & 47.30 & 47.38 & 47.46 \\
\hline Line.134 & 1 & 9.99 & 9.93 & 9.79 & 9.78 & 9.77 \\
\hline Line.134 & 2 & 9.99 & 9.93 & 9.78 & 9.78 & 9.77 \\
\hline Line. 135 & 1 & 315.13 & 320.25 & 316.33 & 312.87 & 307.03 \\
\hline
\end{tabular}


Table 4-20: $I_{1}$ value, zone 3,3 phase fault, $50 \%$ penetration

\begin{tabular}{|c|c|c|c|c|c|c|}
\hline Element & Terminal & \multicolumn{5}{|c|}{ Faulty Node } \\
\hline & & $\mathbf{7 3 4}$ & $\mathbf{7 1 0}$ & $\mathbf{7 3 7}$ & $\mathbf{7 3 8}$ & $\mathbf{7 1 1}$ \\
\hline Vsource & 1 & 15.68 & 15.16 & 15.24 & 14.98 & 14.73 \\
\hline Vsource & 2 & 15.68 & 15.16 & 15.24 & 14.98 & 14.73 \\
\hline Subxf & 1 & 15.68 & 15.16 & 15.24 & 14.98 & 14.73 \\
\hline Subxf & 2 & 751.45 & 726.45 & 730.24 & 717.89 & 706.03 \\
\hline Xfm1 & 1 & 0.00 & 0.00 & 0.00 & 0.00 & 0.00 \\
\hline Xfm1 & 2 & 0.00 & 0.00 & 0.00 & 0.00 & 0.00 \\
\hline Line.11 & 1 & 680.44 & 657.38 & 660.96 & 649.62 & 638.72 \\
\hline Line.11 & 2 & 680.45 & 657.39 & 660.97 & 649.62 & 638.73 \\
\hline Line.12 & 1 & 49.19 & 46.75 & 47.22 & 46.07 & 44.97 \\
\hline Line.12 & 2 & 49.19 & 46.75 & 47.22 & 46.07 & 44.96 \\
\hline Line.13 & 1 & 82.89 & 78.02 & 79.19 & 77.02 & 74.95 \\
\hline Line.13 & 2 & 82.89 & 78.01 & 79.18 & 77.02 & 74.94 \\
\hline Line.14 & 1 & 810.86 & 780.51 & 785.59 & 770.86 & 756.71 \\
\hline Line.14 & 2 & 810.87 & 780.51 & 785.59 & 770.87 & 756.72 \\
\hline Line.15 & 1 & 86.15 & 81.69 & 82.67 & 80.63 & 78.67 \\
\hline Line.15 & 2 & 86.15 & 81.69 & 82.66 & 80.63 & 78.67 \\
\hline Line.16 & 1 & 38.55 & 36.13 & 36.85 & 35.85 & 34.90 \\
\hline Line.16 & 2 & 38.54 & 36.13 & 36.84 & 35.85 & 34.89 \\
\hline Line.17 & 1 & 1.76 & 1.99 & 1.91 & 1.99 & 2.07 \\
\hline Line.17 & 2 & 1.76 & 1.99 & 1.91 & 1.99 & 2.07 \\
\hline Line.18 & 1 & 1.76 & 1.99 & 1.91 & 1.99 & 2.07 \\
\hline Line.18 & 2 & 1.76 & 1.99 & 1.91 & 1.99 & 2.07 \\
\hline Line.19 & 1 & 43.27 & 41.41 & 41.83 & 40.99 & 40.17 \\
\hline Line.19 & 2 & 43.26 & 41.40 & 41.82 & 40.98 & 40.17 \\
\hline Line.110 & 1 & 19.36 & 19.36 & 19.36 & 19.36 & 19.36 \\
\hline Line.110 & 2 & 19.36 & 19.36 & 19.36 & 19.36 & 19.36 \\
\hline Line.111 & 1 & 61.29 & 59.39 & 59.65 & 58.69 & 57.77 \\
\hline Line.111 & 2 & 61.29 & 59.39 & 59.64 & 58.69 & 57.77 \\
\hline Line.112 & 1 & 48.17 & 46.22 & 46.50 & 45.53 & 44.61 \\
\hline Line.112 & 2 & 48.17 & 46.22 & 46.50 & 45.53 & 44.61 \\
\hline Line.113 & 1 & 52.57 & 50.63 & 50.88 & 49.90 & 48.96 \\
\hline Line.113 & 2 & 52.57 & 50.63 & 50.88 & 49.90 & 48.96 \\
\hline Line.114 & 1 & 3.59 & 4.05 & 3.90 & 4.08 & 4.26 \\
\hline Line.114 & 2 & 3.59 & 4.05 & 3.90 & 4.08 & 4.26 \\
\hline Line.115 & 1 & 52.61 & 50.62 & 50.90 & 49.91 & 48.96 \\
\hline Line.115 & 2 & 52.61 & 50.62 & 50.90 & 49.91 & 48.96 \\
\hline Line.116 & 1 & 55.22 & 52.30 & 52.97 & 51.65 & 50.39 \\
\hline
\end{tabular}




\begin{tabular}{|l|c|c|c|c|c|c|}
\hline Element & Terminal & \multicolumn{5}{|c|}{ Faulty Node } \\
\hline & & $\mathbf{7 3 4}$ & $\mathbf{7 1 0}$ & $\mathbf{7 3 7}$ & $\mathbf{7 3 8}$ & $\mathbf{7 1 1}$ \\
\hline Line.116 & 2 & 55.22 & 52.30 & 52.97 & 51.65 & 50.39 \\
\hline Line.117 & 1 & 865.23 & 831.92 & 837.65 & 821.57 & 806.13 \\
\hline Line.117 & 2 & 865.24 & 831.92 & 837.65 & 821.57 & 806.14 \\
\hline Line.118 & 1 & 862.67 & 828.93 & 834.83 & 818.59 & 803.01 \\
\hline Line.118 & 2 & 862.67 & 828.93 & 834.83 & 818.60 & 803.01 \\
\hline Line.119 & 1 & 59.88 & 57.66 & 57.96 & 56.85 & 55.79 \\
\hline Line.119 & 2 & 59.88 & 57.66 & 57.96 & 56.85 & 55.78 \\
\hline Line.120 & 1 & 922.33 & 886.42 & 892.61 & 875.29 & 858.66 \\
\hline Line.120 & 2 & 922.33 & 886.42 & 892.61 & 875.29 & 858.66 \\
\hline Line.121 & 1 & 1.26 & 1.51 & 1.42 & 1.52 & 1.61 \\
\hline Line.121 & 2 & 1.26 & 1.51 & 1.42 & 1.52 & 1.61 \\
\hline Line.122 & 1 & 921.08 & 884.93 & 891.21 & 873.80 & 857.09 \\
\hline Line.122 & 2 & 921.08 & 884.93 & 891.21 & 873.80 & 857.09 \\
\hline Line.123 & 1 & 56.72 & 54.01 & 54.58 & 53.33 & 52.13 \\
\hline Line.123 & 2 & 56.72 & 54.01 & 54.58 & 53.33 & 52.13 \\
\hline Line.124 & 1 & 62.90 & 61.04 & 61.26 & 60.30 & 59.38 \\
\hline Line.124 & 2 & 62.90 & 61.04 & 61.26 & 60.30 & 59.38 \\
\hline Line.125 & 1 & 4.87 & 5.58 & 5.33 & 5.61 & 5.87 \\
\hline Line.125 & 2 & 4.87 & 5.58 & 5.33 & 5.61 & 5.87 \\
\hline Line.126 & 1 & 910.88 & 874.77 & 881.08 & 863.72 & 847.05 \\
\hline Line.126 & 2 & 910.88 & 874.77 & 881.08 & 863.72 & 847.05 \\
\hline Line.127 & 1 & 39.30 & 36.52 & 940.80 & 922.07 & 904.08 \\
\hline Line.127 & 2 & 39.29 & 36.52 & 940.80 & 922.07 & 904.08 \\
\hline Line.128 & 1 & 55.85 & 52.75 & 56.00 & 905.26 & 887.34 \\
\hline Line.128 & 2 & 55.85 & 52.75 & 56.00 & 905.26 & 887.34 \\
\hline Line.129 & 1 & 58.90 & 56.50 & 58.93 & 58.98 & 884.15 \\
\hline Line.129 & 2 & 58.90 & 56.50 & 58.93 & 58.98 & 884.15 \\
\hline Line.130 & 1 & 60.98 & 59.05 & 60.90 & 60.90 & 60.90 \\
\hline Line.130 & 2 & 60.98 & 59.05 & 60.90 & 60.90 & 60.90 \\
\hline Line.131 & 1 & 2.09 & 2.59 & 2.00 & 1.94 & 1.89 \\
\hline Line.131 & 2 & 2.09 & 2.59 & 2.00 & 1.94 & 1.89 \\
\hline Line.132 & 1 & 63.55 & 908.87 & 61.31 & 60.00 & 58.75 \\
\hline Line.132 & 2 & 63.55 & 908.87 & 61.31 & 60.00 & 58.75 \\
\hline Line.133 & 1 & 65.62 & 65.47 & 63.66 & 62.52 & 61.43 \\
\hline Line.133 & 2 & 65.62 & 65.47 & 63.66 & 62.52 & 61.43 \\
\hline Line.134 & 1 & 2.08 & 1.96 & 2.40 & 2.59 & 2.78 \\
\hline Line.134 & 2 & 2.08 & 1.96 & 2.40 & 2.59 & 2.78 \\
\hline
\end{tabular}


Table 4-21: Ir value, zone 3, SLG fault, $50 \%$ penetration

\begin{tabular}{|c|c|c|c|c|c|c|}
\hline Element & Terminal & \multicolumn{5}{|c|}{ Faulty Node } \\
\hline & & $\mathbf{7 3 4}$ & $\mathbf{7 1 0}$ & $\mathbf{7 3 7}$ & $\mathbf{7 3 8}$ & $\mathbf{7 1 1}$ \\
\hline Vsource & 1 & 0.00 & 0.00 & 0.00 & 0.00 & 0.00 \\
\hline Vsource & 2 & 0.00 & 0.00 & 0.00 & 0.00 & 0.00 \\
\hline Subxf & 1 & 0.00 & 0.00 & 0.00 & 0.00 & 0.00 \\
\hline Subxf & 2 & 0.00 & 0.00 & 0.00 & 0.00 & 0.00 \\
\hline Xfm1 & 1 & 0.00 & 0.00 & 0.00 & 0.00 & 0.00 \\
\hline Xfm1 & 2 & 0.00 & 0.00 & 0.00 & 0.00 & 0.00 \\
\hline Line.11 & 1 & 0.20 & 0.19 & 0.19 & 0.19 & 0.18 \\
\hline Line.11 & 2 & 0.20 & 0.19 & 0.19 & 0.19 & 0.18 \\
\hline Line.12 & 1 & 119.36 & 114.04 & 114.64 & 111.79 & 109.02 \\
\hline Line.12 & 2 & 119.36 & 114.04 & 114.64 & 111.79 & 109.02 \\
\hline Line.13 & 1 & 234.95 & 224.47 & 225.65 & 220.04 & 214.59 \\
\hline Line.13 & 2 & 234.95 & 224.47 & 225.65 & 220.04 & 214.59 \\
\hline Line.14 & 1 & 353.97 & 338.18 & 339.97 & 331.51 & 323.30 \\
\hline Line.14 & 2 & 353.97 & 338.18 & 339.97 & 331.51 & 323.30 \\
\hline Line.15 & 1 & 234.96 & 224.49 & 225.67 & 220.05 & 214.61 \\
\hline Line.15 & 2 & 234.96 & 224.49 & 225.67 & 220.05 & 214.61 \\
\hline Line.16 & 1 & 117.02 & 111.80 & 112.39 & 109.59 & 106.88 \\
\hline Line.16 & 2 & 117.02 & 111.80 & 112.39 & 109.59 & 106.88 \\
\hline Line.17 & 1 & 0.04 & 0.04 & 0.04 & 0.04 & 0.04 \\
\hline Line.17 & 2 & 0.04 & 0.04 & 0.04 & 0.04 & 0.04 \\
\hline Line.18 & 1 & 0.01 & 0.01 & 0.01 & 0.01 & 0.01 \\
\hline Line.18 & 2 & 0.01 & 0.01 & 0.01 & 0.01 & 0.01 \\
\hline Line.19 & 1 & 117.10 & 111.88 & 112.47 & 109.67 & 106.95 \\
\hline Line.19 & 2 & 117.10 & 111.88 & 112.47 & 109.67 & 106.95 \\
\hline Line.110 & 1 & 0.00 & 0.00 & 0.00 & 0.00 & 0.00 \\
\hline Line.110 & 2 & 0.00 & 0.00 & 0.00 & 0.00 & 0.00 \\
\hline Line.111 & 1 & 117.14 & 111.92 & 112.51 & 109.71 & 106.99 \\
\hline Line.111 & 2 & 117.14 & 111.92 & 112.51 & 109.71 & 106.99 \\
\hline Line.112 & 1 & 118.00 & 112.74 & 113.33 & 110.51 & 107.78 \\
\hline Line.112 & 2 & 118.00 & 112.74 & 113.33 & 110.51 & 107.78 \\
\hline Line.113 & 1 & 118.01 & 112.74 & 113.34 & 110.52 & 107.78 \\
\hline Line.113 & 2 & 118.01 & 112.74 & 113.34 & 110.52 & 107.78 \\
\hline Line.114 & 1 & 0.01 & 0.01 & 0.01 & 0.01 & 0.01 \\
\hline Line.114 & 2 & 0.01 & 0.01 & 0.01 & 0.01 & 0.01 \\
\hline Line.115 & 1 & 119.39 & 114.06 & 114.66 & 111.81 & 109.04 \\
\hline Line.115 & 2 & 119.39 & 114.06 & 114.66 & 111.81 & 109.04 \\
\hline Line.116 & 1 & 126.46 & 120.82 & 121.46 & 118.44 & 115.50 \\
\hline Line.116 & 2 & 126.46 & 120.82 & 121.46 & 118.44 & 115.50 \\
\hline
\end{tabular}




\begin{tabular}{|l|c|c|c|c|c|c|}
\hline Element & Terminal & \multicolumn{5}{|c|}{ Faulty Node } \\
\hline & & $\mathbf{7 3 4}$ & $\mathbf{7 1 0}$ & $\mathbf{7 3 7}$ & $\mathbf{7 3 8}$ & $\mathbf{7 1 1}$ \\
\hline Line.117 & 1 & 480.23 & 458.81 & 461.23 & 449.76 & 438.62 \\
\hline Line.117 & 2 & 480.23 & 458.81 & 461.23 & 449.76 & 438.62 \\
\hline Line.118 & 1 & 480.20 & 458.78 & 461.20 & 449.73 & 438.59 \\
\hline Line.118 & 2 & 480.20 & 458.78 & 461.20 & 449.73 & 438.59 \\
\hline Line.119 & 1 & 130.28 & 124.47 & 125.12 & 122.01 & 118.99 \\
\hline Line.119 & 2 & 130.28 & 124.47 & 125.12 & 122.01 & 118.99 \\
\hline Line.120 & 1 & 610.12 & 582.91 & 585.99 & 571.41 & 557.26 \\
\hline Line.120 & 2 & 610.12 & 582.91 & 585.99 & 571.41 & 557.26 \\
\hline Line.121 & 1 & 0.01 & 0.01 & 0.01 & 0.01 & 0.01 \\
\hline Line.121 & 2 & 0.01 & 0.01 & 0.01 & 0.01 & 0.01 \\
\hline Line.122 & 1 & 610.09 & 582.88 & 585.96 & 571.38 & 557.23 \\
\hline Line.122 & 2 & 610.09 & 582.88 & 585.96 & 571.38 & 557.23 \\
\hline Line.123 & 1 & 126.47 & 120.83 & 121.47 & 118.44 & 115.51 \\
\hline Line.123 & 2 & 126.47 & 120.83 & 121.47 & 118.44 & 115.51 \\
\hline Line.124 & 1 & 126.49 & 120.85 & 121.49 & 118.47 & 115.53 \\
\hline Line.124 & 2 & 126.49 & 120.85 & 121.49 & 118.47 & 115.53 \\
\hline Line.125 & 1 & 0.01 & 0.01 & 0.01 & 0.01 & 0.01 \\
\hline Line.125 & 2 & 0.01 & 0.01 & 0.01 & 0.01 & 0.01 \\
\hline Line.126 & 1 & 610.08 & 582.87 & 585.94 & 571.37 & 557.22 \\
\hline Line.126 & 2 & 610.08 & 582.87 & 585.94 & 571.37 & 557.22 \\
\hline Line.127 & 1 & 137.70 & 131.55 & 718.05 & 700.19 & 682.85 \\
\hline Line.127 & 2 & 137.70 & 131.55 & 718.05 & 700.19 & 682.85 \\
\hline Line.128 & 1 & 137.73 & 131.59 & 137.21 & 700.15 & 682.82 \\
\hline Line.128 & 2 & 137.73 & 131.59 & 137.21 & 700.15 & 682.82 \\
\hline Line.129 & 1 & 137.75 & 131.61 & 137.23 & 136.98 & 682.80 \\
\hline Line.129 & 2 & 137.75 & 131.61 & 137.23 & 136.98 & 682.80 \\
\hline Line.130 & 1 & 137.78 & 131.64 & 137.26 & 137.01 & 136.82 \\
\hline Line.130 & 2 & 137.78 & 131.64 & 137.26 & 137.01 & 136.82 \\
\hline Line.131 & 1 & 0.01 & 0.01 & 0.01 & 0.01 & 0.01 \\
\hline Line.131 & 2 & 0.01 & 0.01 & 0.01 & 0.01 & 0.01 \\
\hline Line.132 & 1 & 138.30 & 713.58 & 132.82 & 129.52 & 126.31 \\
\hline Line.132 & 2 & 138.30 & 713.58 & 132.82 & 129.52 & 126.31 \\
\hline Line.133 & 1 & 138.33 & 137.21 & 132.86 & 129.55 & 126.34 \\
\hline Line.133 & 2 & 138.33 & 137.21 & 132.86 & 129.55 & 126.34 \\
\hline Line.134 & 1 & 0.01 & 0.01 & 0.01 & 0.01 & 0.01 \\
\hline Line.134 & 2 & 0.01 & 0.01 & 0.01 & 0.01 & 0.01 \\
\hline & & & & & & \\
\hline
\end{tabular}


Table 4-22: $I_{2}$ value, zone 3 , LL fault, $50 \%$ penetration

\begin{tabular}{|c|c|c|c|c|c|c|}
\hline Element & Terminal & \multicolumn{5}{|c|}{ Faulty Node } \\
\hline & & $\mathbf{7 3 4}$ & $\mathbf{7 1 0}$ & $\mathbf{7 3 7}$ & $\mathbf{7 3 8}$ & $\mathbf{7 1 1}$ \\
\hline Vsource & 1 & 6.70 & 6.41 & 6.46 & 6.32 & 6.18 \\
\hline Vsource & 2 & 6.70 & 6.41 & 6.46 & 6.32 & 6.18 \\
\hline Subxf & 1 & 6.70 & 6.41 & 6.46 & 6.32 & 6.18 \\
\hline Subxf & 2 & 321.26 & 307.27 & 309.53 & 302.82 & 296.36 \\
\hline Xfm1 & 1 & 0.00 & 0.00 & 0.00 & 0.00 & 0.00 \\
\hline Xfm1 & 2 & 0.00 & 0.00 & 0.00 & 0.00 & 0.00 \\
\hline Line.11 & 1 & 292.26 & 279.57 & 281.59 & 275.49 & 269.62 \\
\hline Line.11 & 2 & 292.24 & 279.55 & 281.57 & 275.46 & 269.59 \\
\hline Line.12 & 1 & 40.14 & 38.93 & 39.03 & 38.40 & 37.79 \\
\hline Line.12 & 2 & 40.15 & 38.94 & 39.04 & 38.40 & 37.79 \\
\hline Line.13 & 1 & 87.28 & 84.82 & 85.02 & 83.71 & 82.44 \\
\hline Line.13 & 2 & 87.28 & 84.82 & 85.02 & 83.72 & 82.44 \\
\hline Line.14 & 1 & 419.07 & 402.76 & 405.06 & 397.03 & 389.29 \\
\hline Line.14 & 2 & 419.04 & 402.73 & 405.02 & 397.00 & 389.26 \\
\hline Line.15 & 1 & 87.74 & 85.14 & 85.46 & 84.14 & 82.86 \\
\hline Line.15 & 2 & 87.75 & 85.15 & 85.47 & 84.15 & 82.86 \\
\hline Line.16 & 1 & 56.86 & 55.97 & 55.96 & 55.44 & 54.92 \\
\hline Line.16 & 2 & 56.87 & 55.98 & 55.97 & 55.45 & 54.93 \\
\hline Line.17 & 1 & 4.15 & 4.12 & 4.14 & 4.14 & 4.14 \\
\hline Line.17 & 2 & 4.16 & 4.12 & 4.15 & 4.15 & 4.15 \\
\hline Line.18 & 1 & 4.16 & 4.12 & 4.15 & 4.15 & 4.15 \\
\hline Line.18 & 2 & 4.16 & 4.13 & 4.15 & 4.15 & 4.15 \\
\hline Line.19 & 1 & 52.90 & 51.95 & 52.00 & 51.47 & 50.95 \\
\hline Line.19 & 2 & 52.92 & 51.96 & 52.01 & 51.48 & 50.96 \\
\hline Line.110 & 1 & 17.68 & 17.55 & 17.58 & 17.53 & 17.47 \\
\hline Line.110 & 2 & 17.68 & 17.55 & 17.58 & 17.53 & 17.48 \\
\hline Line.111 & 1 & 38.56 & 37.13 & 37.35 & 36.65 & 35.98 \\
\hline Line.111 & 2 & 38.57 & 37.13 & 37.36 & 36.66 & 35.99 \\
\hline Line.112 & 1 & 34.14 & 32.28 & 32.72 & 31.90 & 31.11 \\
\hline Line.112 & 2 & 34.15 & 32.28 & 32.72 & 31.90 & 31.11 \\
\hline Line.113 & 1 & 33.82 & 31.86 & 32.30 & 31.42 & 30.58 \\
\hline Line.113 & 2 & 33.83 & 31.87 & 32.30 & 31.43 & 30.59 \\
\hline Line.114 & 1 & 10.27 & 10.46 & 10.38 & 10.44 & 10.49 \\
\hline Line.114 & 2 & 10.27 & 10.46 & 10.38 & 10.44 & 10.49 \\
\hline Line.115 & 1 & 42.03 & 40.65 & 40.87 & 40.20 & 39.56 \\
\hline Line.115 & 2 & 42.03 & 40.66 & 40.87 & 40.20 & 39.56 \\
\hline Line.116 & 1 & 33.27 & 31.36 & 31.74 & 30.85 & 30.00 \\
\hline
\end{tabular}




\begin{tabular}{|l|c|c|c|c|c|c|}
\hline Element & Terminal & \multicolumn{5}{|c|}{ Faulty Node } \\
\hline & & $\mathbf{7 3 4}$ & $\mathbf{7 1 0}$ & $\mathbf{7 3 7}$ & $\mathbf{7 3 8}$ & $\mathbf{7 1 1}$ \\
\hline Line.116 & 2 & 33.27 & 31.36 & 31.74 & 30.86 & 30.01 \\
\hline Line.117 & 1 & 452.06 & 433.86 & 436.51 & 427.60 & 419.01 \\
\hline Line.117 & 2 & 452.05 & 433.85 & 436.50 & 427.59 & 419.00 \\
\hline Line.118 & 1 & 451.66 & 433.65 & 436.16 & 427.28 & 418.72 \\
\hline Line.118 & 2 & 451.66 & 433.64 & 436.16 & 427.28 & 418.72 \\
\hline Line.119 & 1 & 47.05 & 45.53 & 45.75 & 45.01 & 44.29 \\
\hline Line.119 & 2 & 47.06 & 45.54 & 45.76 & 45.02 & 44.30 \\
\hline Line.120 & 1 & 496.94 & 477.36 & 480.13 & 470.50 & 461.22 \\
\hline Line.120 & 2 & 496.93 & 477.35 & 480.13 & 470.50 & 461.22 \\
\hline Line.121 & 1 & 4.90 & 5.01 & 4.96 & 5.00 & 5.03 \\
\hline Line.121 & 2 & 4.90 & 5.01 & 4.96 & 4.99 & 5.03 \\
\hline Line.122 & 1 & 496.74 & 477.25 & 479.96 & 470.34 & 461.08 \\
\hline Line.122 & 2 & 496.73 & 477.25 & 479.95 & 470.34 & 461.08 \\
\hline Line.123 & 1 & 34.56 & 32.59 & 33.06 & 32.19 & 31.35 \\
\hline Line.123 & 2 & 34.57 & 32.60 & 33.07 & 32.20 & 31.36 \\
\hline Line.124 & 1 & 35.54 & 33.83 & 34.22 & 33.45 & 32.70 \\
\hline Line.124 & 2 & 35.55 & 33.84 & 34.22 & 33.45 & 32.71 \\
\hline Line.125 & 1 & 6.72 & 6.42 & 6.47 & 6.33 & 6.20 \\
\hline Line.125 & 2 & 6.72 & 6.42 & 6.47 & 6.33 & 6.20 \\
\hline Line.126 & 1 & 486.58 & 467.17 & 469.95 & 460.41 & 451.22 \\
\hline Line.126 & 2 & 486.57 & 467.16 & 469.94 & 460.41 & 451.21 \\
\hline Line.127 & 1 & 22.34 & 20.35 & 512.94 & 502.61 & 492.64 \\
\hline Line.127 & 2 & 22.35 & 20.36 & 512.93 & 502.60 & 492.63 \\
\hline Line.128 & 1 & 39.02 & 36.59 & 39.16 & 485.83 & 476.00 \\
\hline Line.128 & 2 & 39.03 & 36.59 & 39.17 & 485.82 & 475.99 \\
\hline Line.129 & 1 & 40.74 & 39.14 & 40.80 & 40.89 & 474.07 \\
\hline Line.129 & 2 & 40.74 & 39.14 & 40.81 & 40.90 & 474.07 \\
\hline Line.130 & 1 & 43.02 & 41.18 & 43.14 & 43.27 & 43.40 \\
\hline Line.130 & 2 & 43.03 & 41.18 & 43.15 & 43.28 & 43.41 \\
\hline Line.131 & 1 & 9.74 & 9.96 & 9.74 & 9.74 & 9.74 \\
\hline Line.131 & 2 & 9.74 & 9.96 & 9.74 & 9.74 & 9.74 \\
\hline Line.132 & 1 & 45.04 & 486.84 & 43.54 & 42.69 & 41.87 \\
\hline Line.132 & 2 & 45.04 & 486.83 & 43.55 & 42.70 & 41.88 \\
\hline Line.133 & 1 & 47.71 & 47.92 & 46.18 & 45.31 & 44.47 \\
\hline Line.133 & 2 & 47.72 & 47.94 & 46.20 & 45.33 & 44.49 \\
\hline Line.134 & 1 & 9.75 & 9.74 & 9.88 & 9.95 & 10.01 \\
\hline Line.134 & 2 & 9.75 & 9.74 & 9.88 & 9.95 & 10.01 \\
\hline
\end{tabular}


Table 4-23: Threshold values, 50\% penetration

\begin{tabular}{|c|c|c|c|}
\hline Zone \# & $\mathbf{I}_{\mathbf{1}}$ th & $\mathbf{I}_{\mathbf{r}}$ th & $\mathbf{I}_{\mathbf{2}}$ th \\
\hline 1 & 920 & 650 & 470 \\
\hline 2 & 900 & 500 & 470 \\
\hline 3 & 880 & 600 & 480 \\
\hline
\end{tabular}

\subsubsection{Short Circuit Analysis 100\% DG Penetration}

Table 4-24: $I_{1}$ value, zone 1, 3 phase fault, $100 \%$ penetration

\begin{tabular}{|c|c|c|c|c|c|c|c|c|}
\hline Element & Terminal & \multicolumn{7}{|c|}{ Faulty Node } \\
\hline & & $\mathbf{7 0 5}$ & $\mathbf{7 1 3}$ & $\mathbf{7 0 4}$ & $\mathbf{7 1 4}$ & $\mathbf{7 2 0}$ & $\mathbf{7 0 6}$ & $\mathbf{7 0 7}$ \\
\hline Vsource & 1 & 0.00 & 0.00 & 0.00 & 0.00 & 0.00 & 0.00 & 0.00 \\
\hline Vsource & 2 & 0.00 & 0.00 & 0.00 & 0.00 & 0.00 & 0.00 & 0.00 \\
\hline Subxf & 1 & 0.00 & 0.00 & 0.00 & 0.00 & 0.00 & 0.00 & 0.00 \\
\hline Subxf & 2 & 0.00 & 0.00 & 0.00 & 0.00 & 0.00 & 0.00 & 0.00 \\
\hline Xfm1 & 1 & 0.00 & 0.00 & 0.00 & 0.00 & 0.00 & 0.00 & 0.00 \\
\hline Xfm1 & 2 & 0.00 & 0.00 & 0.00 & 0.00 & 0.00 & 0.00 & 0.00 \\
\hline Line.11 & 1 & 1.24 & 1.12 & 1.24 & 1.28 & 1.45 & 1.62 & 1.87 \\
\hline Line.11 & 2 & 1.24 & 1.12 & 1.24 & 1.28 & 1.45 & 1.62 & 1.87 \\
\hline Line.12 & 1 & 581.71 & 90.88 & 89.71 & 89.41 & 87.63 & 85.91 & 84.33 \\
\hline Line.12 & 2 & 581.71 & 90.88 & 89.71 & 89.41 & 87.63 & 85.91 & 84.33 \\
\hline Line.13 & 1 & 171.43 & 504.55 & 497.89 & 496.16 & 486.04 & 476.23 & 466.71 \\
\hline Line.13 & 2 & 171.43 & 504.55 & 497.89 & 496.16 & 486.04 & 476.23 & 466.71 \\
\hline Line.14 & 1 & 411.54 & 414.80 & 409.42 & 408.03 & 399.84 & 391.91 & 384.23 \\
\hline Line.14 & 2 & 411.54 & 414.80 & 409.42 & 408.03 & 399.84 & 391.91 & 384.22 \\
\hline Line.15 & 1 & 173.16 & 176.35 & 496.31 & 494.54 & 484.19 & 474.17 & 464.30 \\
\hline Line.15 & 2 & 173.16 & 176.35 & 496.31 & 494.54 & 484.19 & 474.17 & 464.30 \\
\hline Line.16 & 1 & 86.24 & 88.01 & 88.38 & 88.07 & 571.04 & 559.50 & 548.41 \\
\hline Line.16 & 2 & 86.24 & 88.01 & 88.38 & 88.07 & 571.04 & 559.50 & 548.41 \\
\hline Line.17 & 1 & 0.88 & 0.73 & 0.70 & 0.72 & 0.66 & 646.78 & 0.95 \\
\hline Line.17 & 2 & 0.88 & 0.73 & 0.70 & 0.72 & 0.66 & 646.78 & 0.95 \\
\hline Line.18 & 1 & 0.88 & 0.73 & 0.70 & 0.72 & 0.66 & 0.65 & 0.95 \\
\hline
\end{tabular}




\begin{tabular}{|c|c|c|c|c|c|c|c|c|}
\hline \multirow[t]{2}{*}{ Element } & \multirow[t]{2}{*}{ Terminal } & \multicolumn{7}{|c|}{ Faulty Node } \\
\hline & & 705 & 713 & 704 & 714 & 720 & 706 & 707 \\
\hline Line.18 & 2 & 0.88 & 0.73 & 0.70 & 0.72 & 0.66 & 0.65 & 0.95 \\
\hline Line.19 & 1 & 88.93 & 90.25 & 90.51 & 90.28 & 90.65 & 89.25 & 545.52 \\
\hline Line.19 & 2 & 88.93 & 90.25 & 90.51 & 90.28 & 90.65 & 89.25 & 545.52 \\
\hline Line. 110 & 1 & 19.36 & 19.37 & 19.37 & 19.37 & 19.37 & 19.37 & 19.36 \\
\hline Line.110 & 2 & 19.36 & 19.37 & 19.37 & 19.37 & 19.37 & 19.37 & 19.36 \\
\hline Line.111 & 1 & 108.12 & 109.53 & 109.81 & 109.56 & 109.93 & 108.32 & 109.94 \\
\hline Line.111 & 2 & 108.12 & 109.53 & 109.81 & 109.56 & 109.93 & 108.32 & 109.94 \\
\hline Line.112 & 1 & 87.02 & 88.41 & 88.70 & 582.43 & 86.86 & 85.34 & 84.12 \\
\hline Line.112 & 2 & 87.02 & 88.41 & 88.70 & 582.43 & 86.86 & 85.34 & 84.12 \\
\hline Line.113 & 1 & 91.58 & 92.98 & 93.26 & 93.27 & 91.40 & 89.85 & 88.63 \\
\hline Line.113 & 2 & 91.58 & 92.98 & 93.26 & 93.27 & 91.40 & 89.85 & 88.63 \\
\hline Line.114 & 1 & 1.40 & 1.58 & 1.75 & 1.80 & 2.03 & 2.27 & 2.61 \\
\hline Line.114 & 2 & 1.40 & 1.58 & 1.75 & 1.80 & 2.03 & 2.27 & 2.61 \\
\hline Line. 115 & 1 & 93.19 & 92.46 & 91.45 & 91.21 & 89.64 & 88.14 & 86.90 \\
\hline Line. 115 & 2 & 93.19 & 92.46 & 91.45 & 91.21 & 89.64 & 88.14 & 86.90 \\
\hline Line.116 & 1 & 114.36 & 115.25 & 113.82 & 113.45 & 111.29 & 109.18 & 107.12 \\
\hline Line.116 & 2 & 114.36 & 115.25 & 113.82 & 113.45 & 111.29 & 109.18 & 107.12 \\
\hline Line.117 & 1 & 297.30 & 299.67 & 295.72 & 294.70 & 288.68 & 282.85 & 277.25 \\
\hline Line.117 & 2 & 297.30 & 299.67 & 295.72 & 294.70 & 288.68 & 282.85 & 277.25 \\
\hline Line.118 & 1 & 299.09 & 301.32 & 297.51 & 296.54 & 290.71 & 285.07 & 279.77 \\
\hline Line. 118 & 2 & 299.09 & 301.32 & 297.51 & 296.54 & 290.70 & 285.07 & 279.77 \\
\hline Line.119 & 1 & 121.64 & 122.27 & 121.07 & 120.78 & 118.92 & 117.13 & 115.60 \\
\hline Line.119 & 2 & 121.64 & 122.27 & 121.07 & 120.78 & 118.92 & 117.13 & 115.60 \\
\hline Line. 120 & 1 & 177.60 & 179.18 & 176.56 & 175.88 & 171.89 & 168.03 & 164.29 \\
\hline Line. 120 & 2 & 177.60 & 179.18 & 176.56 & 175.88 & 171.89 & 168.03 & 164.29 \\
\hline Line. 121 & 1 & 1.03 & 0.95 & 1.04 & 1.06 & 1.18 & 1.29 & 1.46 \\
\hline Line. 121 & 2 & 1.03 & 0.95 & 1.04 & 1.06 & 1.18 & 1.29 & 1.46 \\
\hline Line.122 & 1 & 178.62 & 180.13 & 177.58 & 176.93 & 173.04 & 169.28 & 165.71 \\
\hline Line.122 & 2 & 178.62 & 180.13 & 177.58 & 176.93 & 173.04 & 169.28 & 165.71 \\
\hline Line. 123 & 1 & 115.29 & 116.10 & 114.75 & 114.40 & 112.34 & 110.33 & 108.44 \\
\hline Line. 123 & 2 & 115.29 & 116.10 & 114.75 & 114.40 & 112.34 & 110.33 & 108.44 \\
\hline Line.124 & 1 & 118.92 & 119.42 & 118.35 & 118.11 & 116.43 & 114.83 & 113.58 \\
\hline Line. 124 & 2 & 118.92 & 119.42 & 118.35 & 118.11 & 116.43 & 114.83 & 113.58 \\
\hline Line. 125 & 1 & 2.77 & 2.53 & 2.77 & 2.85 & 3.17 & 3.51 & 4.01 \\
\hline Line. 125 & 2 & 2.77 & 2.53 & 2.77 & 2.85 & 3.17 & 3.51 & 4.01 \\
\hline Line.126 & 1 & 188.80 & 190.30 & 187.72 & 187.07 & 183.13 & 179.32 & 175.78 \\
\hline Line.126 & 2 & 188.80 & 190.30 & 187.72 & 187.07 & 183.13 & 179.32 & 175.78 \\
\hline Line. 127 & 1 & 82.86 & 83.66 & 82.38 & 82.04 & 80.09 & 78.19 & 76.30 \\
\hline Line. 127 & 2 & 82.86 & 83.66 & 82.37 & 82.04 & 80.09 & 78.18 & 76.30 \\
\hline
\end{tabular}




\begin{tabular}{|c|c|c|c|c|c|c|c|c|}
\hline Element & Terminal & \multicolumn{7}{|c|}{ Faulty Node } \\
\hline & & $\mathbf{7 0 5}$ & $\mathbf{7 1 3}$ & $\mathbf{7 0 4}$ & $\mathbf{7 1 4}$ & $\mathbf{7 2 0}$ & $\mathbf{7 0 6}$ & $\mathbf{7 0 7}$ \\
\hline Line.128 & 1 & 99.57 & 100.36 & 99.02 & 98.68 & 96.63 & 94.65 & 92.81 \\
\hline Line.128 & 2 & 99.57 & 100.36 & 99.02 & 98.68 & 96.63 & 94.64 & 92.81 \\
\hline Line.129 & 1 & 102.70 & 103.25 & 102.13 & 101.87 & 100.12 & 98.46 & 97.09 \\
\hline Line.129 & 2 & 102.70 & 103.25 & 102.13 & 101.87 & 100.12 & 98.46 & 97.09 \\
\hline Line.130 & 1 & 104.97 & 105.38 & 104.41 & 104.20 & 102.67 & 101.22 & 100.18 \\
\hline Line.130 & 2 & 104.97 & 105.38 & 104.41 & 104.20 & 102.67 & 101.21 & 100.18 \\
\hline Line.131 & 1 & 2.30 & 2.14 & 2.31 & 2.36 & 2.60 & 2.83 & 3.16 \\
\hline Line.131 & 2 & 2.30 & 2.14 & 2.31 & 2.36 & 2.60 & 2.83 & 3.16 \\
\hline Line.132 & 1 & 107.07 & 107.69 & 106.49 & 106.20 & 104.33 & 102.56 & 101.06 \\
\hline Line.132 & 2 & 107.07 & 107.69 & 106.49 & 106.20 & 104.33 & 102.55 & 101.06 \\
\hline Line.133 & 1 & 109.32 & 109.79 & 108.75 & 108.51 & 106.86 & 105.30 & 104.14 \\
\hline Line.133 & 2 & 109.32 & 109.79 & 108.75 & 108.51 & 106.86 & 105.30 & 104.14 \\
\hline Line.134 & 1 & 2.27 & 2.12 & 2.28 & 2.34 & 2.57 & 2.80 & 3.13 \\
\hline Line.134 & 2 & 2.27 & 2.12 & 2.28 & 2.34 & 2.57 & 2.80 & 3.13 \\
\hline
\end{tabular}

Table 4-25: $I_{r}$ value, zone 1, SLG fault, $100 \%$ penetration

\begin{tabular}{|c|c|c|c|c|c|c|c|c|}
\hline Element & Terminal & \multicolumn{7}{|c|}{ Faulty Node } \\
\hline & & $\mathbf{7 0 5}$ & $\mathbf{7 1 3}$ & $\mathbf{7 0 4}$ & $\mathbf{7 1 4}$ & $\mathbf{7 2 0}$ & $\mathbf{7 0 6}$ & $\mathbf{7 0 7}$ \\
\hline Vsource & 1 & 0.00 & 0.00 & 0.00 & 0.00 & 0.00 & 0.00 & 0.00 \\
\hline Vsource & 2 & 0.00 & 0.00 & 0.00 & 0.00 & 0.00 & 0.00 & 0.00 \\
\hline Subxf & 1 & 0.00 & 0.00 & 0.00 & 0.00 & 0.00 & 0.00 & 0.00 \\
\hline Subxf & 2 & 0.00 & 0.00 & 0.00 & 0.00 & 0.00 & 0.00 & 0.00 \\
\hline Xfm1 & 1 & 0.00 & 0.00 & 0.00 & 0.00 & 0.00 & 0.00 & 0.00 \\
\hline Xfm1 & 2 & 0.00 & 0.00 & 0.00 & 0.00 & 0.00 & 0.00 & 0.00 \\
\hline Line.11 & 1 & 0.13 & 0.13 & 0.13 & 0.12 & 0.12 & 0.11 & 0.11 \\
\hline Line.11 & 2 & 0.13 & 0.13 & 0.13 & 0.12 & 0.12 & 0.11 & 0.11 \\
\hline Line.12 & 1 & 724.53 & 113.33 & 110.06 & 109.31 & 104.64 & 100.44 & 96.85 \\
\hline Line.12 & 2 & 724.53 & 113.33 & 110.06 & 109.31 & 104.64 & 100.44 & 96.85 \\
\hline Line.13 & 1 & 221.51 & 623.28 & 605.28 & 601.16 & 575.49 & 552.36 & 532.65 \\
\hline Line.13 & 2 & 221.51 & 623.28 & 605.28 & 601.16 & 575.49 & 552.36 & 532.65 \\
\hline Line.14 & 1 & 503.56 & 510.85 & 496.10 & 492.72 & 471.68 & 452.72 & 436.56 \\
\hline Line.14 & 2 & 503.56 & 510.85 & 496.10 & 492.72 & 471.68 & 452.72 & 436.56 \\
\hline Line.15 & 1 & 221.52 & 232.10 & 605.27 & 601.15 & 575.48 & 552.35 & 532.64 \\
\hline Line.15 & 2 & 221.52 & 232.10 & 605.27 & 601.15 & 575.48 & 552.35 & 532.64 \\
\hline Line.16 & 1 & 111.83 & 117.17 & 119.34 & 118.53 & 685.09 & 657.55 & 634.08 \\
\hline Line.16 & 2 & 111.83 & 117.17 & 119.34 & 118.53 & 685.09 & 657.55 & 634.08 \\
\hline Line.17 & 1 & 0.03 & 0.03 & 0.03 & 0.03 & 0.03 & 772.71 & 0.03 \\
\hline
\end{tabular}




\begin{tabular}{|c|c|c|c|c|c|c|c|c|}
\hline \multirow[t]{2}{*}{ Element } & \multirow[t]{2}{*}{ Terminal } & \multicolumn{7}{|c|}{ Faulty Node } \\
\hline & & 705 & 713 & 704 & 714 & 720 & 706 & 707 \\
\hline Line.17 & 2 & 0.03 & 0.03 & 0.03 & 0.03 & 0.03 & 772.71 & 0.03 \\
\hline Line. 18 & 1 & 0.01 & 0.01 & 0.01 & 0.01 & 0.01 & 0.01 & 0.01 \\
\hline Line.18 & 2 & 0.01 & 0.01 & 0.01 & 0.01 & 0.01 & 0.01 & 0.01 \\
\hline Line.19 & 1 & 111.88 & 117.23 & 119.40 & 118.58 & 122.44 & 117.52 & 634.03 \\
\hline Line.19 & 2 & 111.88 & 117.23 & 119.40 & 118.58 & 122.44 & 117.52 & 634.03 \\
\hline Line.110 & 1 & 0.00 & 0.00 & 0.00 & 0.00 & 0.00 & 0.00 & 0.00 \\
\hline Line.110 & 2 & 0.00 & 0.00 & 0.00 & 0.00 & 0.00 & 0.00 & 0.00 \\
\hline Line.111 & 1 & 111.91 & 117.26 & 119.43 & 118.61 & 122.46 & 117.54 & 127.14 \\
\hline Line.111 & 2 & 111.91 & 117.26 & 119.43 & 118.61 & 122.46 & 117.54 & 127.14 \\
\hline Line.112 & 1 & 109.77 & 115.01 & 117.14 & 718.62 & 111.38 & 106.90 & 103.09 \\
\hline Line.112 & 2 & 109.77 & 115.01 & 117.14 & 718.62 & 111.38 & 106.90 & 103.09 \\
\hline Line.113 & 1 & 109.77 & 115.02 & 117.15 & 117.45 & 111.38 & 106.91 & 103.09 \\
\hline Line.113 & 2 & 109.77 & 115.02 & 117.15 & 117.45 & 111.38 & 106.91 & 103.09 \\
\hline Line.114 & 1 & 0.01 & 0.01 & 0.01 & 0.01 & 0.01 & 0.01 & 0.01 \\
\hline Line.114 & 2 & 0.01 & 0.01 & 0.01 & 0.01 & 0.01 & 0.01 & 0.01 \\
\hline Line.115 & 1 & 116.98 & 113.35 & 110.07 & 109.32 & 104.66 & 100.45 & 96.87 \\
\hline Line.115 & 2 & 116.98 & 113.35 & 110.07 & 109.32 & 104.66 & 100.45 & 96.87 \\
\hline Line.116 & 1 & 130.62 & 132.51 & 128.69 & 127.81 & 122.35 & 117.43 & 113.24 \\
\hline Line.116 & 2 & 130.62 & 132.51 & 128.69 & 127.81 & 122.35 & 117.43 & 113.24 \\
\hline Line.117 & 1 & 373.57 & 378.98 & 368.04 & 365.53 & 349.92 & 335.86 & 323.87 \\
\hline Line.117 & 2 & 373.57 & 378.98 & 368.04 & 365.53 & 349.92 & 335.86 & 323.87 \\
\hline Line.118 & 1 & 373.59 & 379.00 & 368.06 & 365.55 & 349.94 & 335.87 & 323.88 \\
\hline Line.118 & 2 & 373.59 & 379.00 & 368.06 & 365.55 & 349.94 & 335.87 & 323.88 \\
\hline Line.119 & 1 & 127.39 & 129.23 & 125.50 & 124.65 & 119.32 & 114.53 & 110.44 \\
\hline Line.119 & 2 & 127.39 & 129.23 & 125.50 & 124.65 & 119.32 & 114.53 & 110.44 \\
\hline Line. 120 & 1 & 246.59 & 250.16 & 242.94 & 241.28 & 230.98 & 221.70 & 213.78 \\
\hline Line. 120 & 2 & 246.59 & 250.16 & 242.94 & 241.28 & 230.98 & 221.70 & 213.78 \\
\hline Line.121 & 1 & 0.01 & 0.01 & 0.01 & 0.01 & 0.01 & 0.01 & 0.01 \\
\hline Line.121 & 2 & 0.01 & 0.01 & 0.01 & 0.01 & 0.01 & 0.01 & 0.01 \\
\hline Line.122 & 1 & 246.61 & 250.18 & 242.96 & 241.30 & 231.00 & 221.71 & 213.80 \\
\hline Line. 122 & 2 & 246.61 & 250.18 & 242.96 & 241.30 & 231.00 & 221.71 & 213.80 \\
\hline Line. 123 & 1 & 130.62 & 132.52 & 128.69 & 127.81 & 122.36 & 117.44 & 113.25 \\
\hline Line. 123 & 2 & 130.62 & 132.52 & 128.69 & 127.81 & 122.36 & 117.44 & 113.25 \\
\hline Line. 124 & 1 & 130.64 & 132.53 & 128.71 & 127.83 & 122.37 & 117.45 & 113.26 \\
\hline Line. 124 & 2 & 130.64 & 132.53 & 128.71 & 127.83 & 122.37 & 117.45 & 113.26 \\
\hline Line.125 & 1 & 0.01 & 0.01 & 0.01 & 0.00 & 0.00 & 0.00 & 0.00 \\
\hline Line.125 & 2 & 0.01 & 0.01 & 0.01 & 0.00 & 0.00 & 0.00 & 0.00 \\
\hline Line.126 & 1 & 246.62 & 250.19 & 242.97 & 241.31 & 231.01 & 221.72 & 213.81 \\
\hline Line.126 & 2 & 246.62 & 250.19 & 242.97 & 241.31 & 231.01 & 221.72 & 213.81 \\
\hline
\end{tabular}




\begin{tabular}{|c|c|c|c|c|c|c|c|c|}
\hline Element & Terminal & \multicolumn{7}{|c|}{ Faulty Node } \\
\hline & & $\mathbf{7 0 5}$ & $\mathbf{7 1 3}$ & $\mathbf{7 0 4}$ & $\mathbf{7 1 4}$ & $\mathbf{7 2 0}$ & $\mathbf{7 0 6}$ & $\mathbf{7 0 7}$ \\
\hline Line.127 & 1 & 123.62 & 125.41 & 121.79 & 120.96 & 115.79 & 111.14 & 107.17 \\
\hline Line.127 & 2 & 123.62 & 125.41 & 121.79 & 120.96 & 115.79 & 111.14 & 107.17 \\
\hline Line.128 & 1 & 123.64 & 125.43 & 121.81 & 120.98 & 115.81 & 111.15 & 107.19 \\
\hline Line.128 & 2 & 123.64 & 125.43 & 121.81 & 120.98 & 115.81 & 111.15 & 107.19 \\
\hline Line.129 & 1 & 123.65 & 125.44 & 121.82 & 120.99 & 115.82 & 111.16 & 107.20 \\
\hline Line.129 & 2 & 123.65 & 125.44 & 121.82 & 120.99 & 115.82 & 111.16 & 107.20 \\
\hline Line.130 & 1 & 123.67 & 125.46 & 121.83 & 121.00 & 115.84 & 111.18 & 107.21 \\
\hline Line.130 & 2 & 123.67 & 125.46 & 121.83 & 121.00 & 115.84 & 111.18 & 107.21 \\
\hline Line.131 & 1 & 0.00 & 0.00 & 0.00 & 0.00 & 0.00 & 0.00 & 0.00 \\
\hline Line.131 & 2 & 0.00 & 0.00 & 0.00 & 0.00 & 0.00 & 0.00 & 0.00 \\
\hline Line.132 & 1 & 123.03 & 124.81 & 121.20 & 120.38 & 115.24 & 110.61 & 106.66 \\
\hline Line.132 & 2 & 123.03 & 124.81 & 121.20 & 120.38 & 115.24 & 110.61 & 106.66 \\
\hline Line.133 & 1 & 123.04 & 124.82 & 121.22 & 120.39 & 115.25 & 110.62 & 106.67 \\
\hline Line.133 & 2 & 123.04 & 124.82 & 121.22 & 120.39 & 115.25 & 110.62 & 106.67 \\
\hline Line.134 & 1 & 0.00 & 0.00 & 0.00 & 0.00 & 0.00 & 0.00 & 0.00 \\
\hline Line.134 & 2 & 0.00 & 0.00 & 0.00 & 0.00 & 0.00 & 0.00 & 0.00 \\
\hline
\end{tabular}

Table 4-26: $I_{2}$ value, zone 1, LL fault, $100 \%$ penetration

\begin{tabular}{|c|c|c|c|c|c|c|c|c|}
\hline Element & Terminal & \multicolumn{7}{|c|}{ Faulty Node } \\
\hline & & $\mathbf{7 0 5}$ & $\mathbf{7 1 3}$ & $\mathbf{7 0 4}$ & $\mathbf{7 1 4}$ & $\mathbf{7 2 0}$ & $\mathbf{7 0 6}$ & $\mathbf{7 0 7}$ \\
\hline Vsource & 1 & 0.00 & 0.00 & 0.00 & 0.00 & 0.00 & 0.00 & 0.00 \\
\hline Vsource & 2 & 0.00 & 0.00 & 0.00 & 0.00 & 0.00 & 0.00 & 0.00 \\
\hline Subxf & 1 & 0.00 & 0.00 & 0.00 & 0.00 & 0.00 & 0.00 & 0.00 \\
\hline Subxf & 2 & 0.00 & 0.00 & 0.00 & 0.00 & 0.00 & 0.00 & 0.00 \\
\hline Xfm1 & 1 & 0.00 & 0.00 & 0.00 & 0.00 & 0.00 & 0.00 & 0.00 \\
\hline Xfm1 & 2 & 0.00 & 0.00 & 0.00 & 0.00 & 0.00 & 0.00 & 0.00 \\
\hline Line.11 & 1 & 4.25 & 4.25 & 4.24 & 4.24 & 4.22 & 4.20 & 4.21 \\
\hline Line.11 & 2 & 4.26 & 4.25 & 4.24 & 4.24 & 4.23 & 4.21 & 4.22 \\
\hline Line.12 & 1 & 331.38 & 56.41 & 55.77 & 55.60 & 54.63 & 53.63 & 52.87 \\
\hline Line.12 & 2 & 331.38 & 56.41 & 55.78 & 55.61 & 54.64 & 53.63 & 52.87 \\
\hline Line.13 & 1 & 120.13 & 270.48 & 266.67 & 265.56 & 259.76 & 253.60 & 248.06 \\
\hline Line.13 & 2 & 120.14 & 270.48 & 266.66 & 265.55 & 259.76 & 253.60 & 248.05 \\
\hline Line.14 & 1 & 211.62 & 214.11 & 210.93 & 209.98 & 205.17 & 200.01 & 195.16 \\
\hline Line.14 & 2 & 211.66 & 214.14 & 210.96 & 210.01 & 205.20 & 200.05 & 195.19 \\
\hline Line.15 & 1 & 121.29 & 123.39 & 265.11 & 264.03 & 258.26 & 252.15 & 246.85 \\
\hline Line.15 & 2 & 121.29 & 123.40 & 265.10 & 264.02 & 258.25 & 252.15 & 246.84 \\
\hline Line.16 & 1 & 71.90 & 72.51 & 72.80 & 72.71 & 309.08 & 301.65 & 294.95 \\
\hline
\end{tabular}




\begin{tabular}{|c|c|c|c|c|c|c|c|c|}
\hline \multirow[t]{2}{*}{ Element } & \multirow[t]{2}{*}{ Terminal } & \multicolumn{7}{|c|}{ Faulty Node } \\
\hline & & 705 & 713 & 704 & 714 & 720 & 706 & 707 \\
\hline Line.16 & 2 & 71.91 & 72.52 & 72.81 & 72.72 & 309.07 & 301.64 & 294.94 \\
\hline Line.17 & 1 & 3.72 & 3.79 & 3.81 & 3.80 & 3.85 & 370.26 & 3.77 \\
\hline Line.17 & 2 & 3.73 & 3.79 & 3.82 & 3.81 & 3.85 & 370.26 & 3.78 \\
\hline Line.18 & 1 & 3.73 & 3.79 & 3.82 & 3.81 & 3.85 & 3.88 & 3.78 \\
\hline Line.18 & 2 & 3.73 & 3.79 & 3.82 & 3.81 & 3.86 & 3.88 & 3.78 \\
\hline Line.19 & 1 & 69.34 & 70.08 & 70.40 & 70.29 & 70.77 & 69.98 & 297.08 \\
\hline Line.19 & 2 & 69.35 & 70.09 & 70.41 & 70.30 & 70.78 & 69.99 & 297.07 \\
\hline Line.110 & 1 & 18.03 & 18.10 & 18.12 & 18.11 & 18.13 & 18.07 & 18.15 \\
\hline Line.110 & 2 & 18.03 & 18.10 & 18.12 & 18.11 & 18.13 & 18.07 & 18.15 \\
\hline Line.111 & 1 & 56.91 & 58.24 & 58.70 & 58.49 & 59.22 & 58.01 & 60.04 \\
\hline Line.111 & 2 & 56.92 & 58.25 & 58.71 & 58.50 & 59.23 & 58.02 & 60.05 \\
\hline Line.112 & 1 & 51.95 & 53.49 & 53.98 & 336.20 & 52.58 & 51.33 & 50.05 \\
\hline Line.112 & 2 & 51.96 & 53.49 & 53.99 & 336.20 & 52.58 & 51.33 & 50.05 \\
\hline Line.113 & 1 & 52.22 & 53.90 & 54.44 & 54.50 & 52.85 & 51.46 & 50.10 \\
\hline Line.113 & 2 & 52.23 & 53.91 & 54.45 & 54.51 & 52.86 & 51.46 & 50.11 \\
\hline Line.114 & 1 & 8.51 & 8.57 & 8.65 & 8.67 & 8.78 & 8.90 & 9.06 \\
\hline Line.114 & 2 & 8.51 & 8.57 & 8.64 & 8.67 & 8.78 & 8.89 & 9.06 \\
\hline Line.115 & 1 & 59.50 & 58.79 & 58.13 & 57.95 & 56.95 & 55.91 & 55.01 \\
\hline Line.115 & 2 & 59.51 & 58.80 & 58.14 & 57.95 & 56.96 & 55.92 & 55.02 \\
\hline Line.116 & 1 & 56.77 & 57.47 & 56.59 & 56.33 & 55.00 & 53.59 & 52.20 \\
\hline Line.116 & 2 & 56.77 & 57.48 & 56.60 & 56.33 & 55.01 & 53.59 & 52.21 \\
\hline Line.117 & 1 & 155.05 & 156.81 & 154.52 & 153.84 & 150.36 & 146.62 & 143.17 \\
\hline Line.117 & 2 & 155.06 & 156.82 & 154.53 & 153.85 & 150.36 & 146.63 & 143.18 \\
\hline Line.118 & 1 & 156.89 & 158.77 & 156.46 & 155.75 & 152.26 & 148.50 & 144.83 \\
\hline Line.118 & 2 & 156.90 & 158.77 & 156.46 & 155.76 & 152.27 & 148.50 & 144.83 \\
\hline Line.119 & 1 & 67.29 & 67.83 & 67.07 & 66.85 & 65.70 & 64.49 & 63.44 \\
\hline Line.119 & 2 & 67.30 & 67.84 & 67.08 & 66.86 & 65.71 & 64.49 & 63.44 \\
\hline Line.120 & 1 & 90.08 & 91.36 & 89.80 & 89.32 & 86.96 & 84.40 & 81.90 \\
\hline Line.120 & 2 & 90.09 & 91.36 & 89.81 & 89.32 & 86.97 & 84.41 & 81.90 \\
\hline Line.121 & 1 & 4.34 & 4.29 & 4.33 & 4.34 & 4.40 & 4.45 & 4.53 \\
\hline Line.121 & 2 & 4.34 & 4.29 & 4.33 & 4.34 & 4.39 & 4.45 & 4.53 \\
\hline Line.122 & 1 & 91.04 & 92.39 & 90.83 & 90.33 & 87.98 & 85.41 & 82.75 \\
\hline Line. 122 & 2 & 91.04 & 92.39 & 90.83 & 90.34 & 87.98 & 85.41 & 82.75 \\
\hline Line. 123 & 1 & 58.15 & 58.90 & 58.02 & 57.75 & 56.44 & 55.02 & 53.56 \\
\hline Line. 123 & 2 & 58.16 & 58.90 & 58.03 & 57.75 & 56.44 & 55.03 & 53.57 \\
\hline Line.124 & 1 & 58.63 & 59.28 & 58.50 & 58.26 & 57.07 & 55.79 & 54.49 \\
\hline Line.124 & 2 & 58.63 & 59.29 & 58.50 & 58.26 & 57.08 & 55.79 & 54.49 \\
\hline Line.125 & 1 & 6.49 & 6.56 & 6.47 & 6.44 & 6.31 & 6.17 & 6.04 \\
\hline Line. 125 & 2 & 6.49 & 6.56 & 6.47 & 6.44 & 6.31 & 6.17 & 6.04 \\
\hline
\end{tabular}




\begin{tabular}{|c|c|c|c|c|c|c|c|c|}
\hline Element & Terminal & \multicolumn{7}{|c|}{ Faulty Node } \\
\hline & & $\mathbf{7 0 5}$ & $\mathbf{7 1 3}$ & $\mathbf{7 0 4}$ & $\mathbf{7 1 4}$ & $\mathbf{7 2 0}$ & $\mathbf{7 0 6}$ & $\mathbf{7 0 7}$ \\
\hline Line.126 & 1 & 100.86 & 102.15 & 100.50 & 100.01 & 97.51 & 94.84 & 92.33 \\
\hline Line.126 & 2 & 100.87 & 102.16 & 100.51 & 100.02 & 97.52 & 94.85 & 92.34 \\
\hline Line.127 & 1 & 39.92 & 40.78 & 39.92 & 39.62 & 38.34 & 36.91 & 35.21 \\
\hline Line.127 & 2 & 39.93 & 40.79 & 39.93 & 39.63 & 38.35 & 36.92 & 35.22 \\
\hline Line.128 & 1 & 55.86 & 56.62 & 55.61 & 55.32 & 53.80 & 52.21 & 50.77 \\
\hline Line.128 & 2 & 55.87 & 56.63 & 55.62 & 55.33 & 53.81 & 52.21 & 50.78 \\
\hline Line.129 & 1 & 58.24 & 58.72 & 57.95 & 57.75 & 56.56 & 55.33 & 54.41 \\
\hline Line.129 & 2 & 58.25 & 58.73 & 57.95 & 57.75 & 56.56 & 55.33 & 54.42 \\
\hline Line.130 & 1 & 60.63 & 61.22 & 60.39 & 60.16 & 58.90 & 57.58 & 56.46 \\
\hline Line.130 & 2 & 60.64 & 61.22 & 60.40 & 60.16 & 58.91 & 57.59 & 56.47 \\
\hline Line.131 & 1 & 8.85 & 8.77 & 8.84 & 8.87 & 8.97 & 9.08 & 9.23 \\
\hline Line.131 & 2 & 8.85 & 8.77 & 8.84 & 8.87 & 8.97 & 9.08 & 9.23 \\
\hline Line.132 & 1 & 62.11 & 62.62 & 61.84 & 61.63 & 60.45 & 59.22 & 58.24 \\
\hline Line.132 & 2 & 62.11 & 62.62 & 61.85 & 61.64 & 60.46 & 59.23 & 58.25 \\
\hline Line.133 & 1 & 64.69 & 65.29 & 64.48 & 64.24 & 63.02 & 61.74 & 60.60 \\
\hline Line.133 & 2 & 64.71 & 65.30 & 64.49 & 64.26 & 63.04 & 61.75 & 60.61 \\
\hline Line.134 & 1 & 8.85 & 8.77 & 8.84 & 8.87 & 8.97 & 9.08 & 9.23 \\
\hline Line.134 & 2 & 8.85 & 8.77 & 8.84 & 8.87 & 8.97 & 9.08 & 9.23 \\
\hline
\end{tabular}

Table 4-27: $I_{1}$ value, zone 2, 3 phase fault, $100 \%$ penetration

\begin{tabular}{|c|c|c|c|c|c|c|}
\hline Element & Terminal & \multicolumn{5}{|c|}{ Faulty Node } \\
\hline & & $\mathbf{7 4 4}$ & $\mathbf{7 2 7}$ & $\mathbf{7 3 0}$ & $\mathbf{7 0 9}$ & $\mathbf{7 0 8}$ \\
\hline Vsource & 1 & 0.00 & 0.00 & 0.00 & 0.00 & 0.00 \\
\hline Vsource & 2 & 0.00 & 0.00 & 0.00 & 0.00 & 0.00 \\
\hline Subxf & 1 & 0.00 & 0.00 & 0.00 & 0.00 & 0.00 \\
\hline Subxf & 2 & 0.00 & 0.00 & 0.00 & 0.00 & 0.00 \\
\hline Xfm1 & 1 & 0.00 & 0.00 & 0.00 & 0.00 & 0.00 \\
\hline Xfm1 & 2 & 0.00 & 0.00 & 0.00 & 0.00 & 0.00 \\
\hline Line.11 & 1 & 1.30 & 1.23 & 1.22 & 1.26 & 1.33 \\
\hline Line.11 & 2 & 1.30 & 1.23 & 1.22 & 1.26 & 1.33 \\
\hline Line.12 & 1 & 89.22 & 89.92 & 89.82 & 89.51 & 88.82 \\
\hline Line.12 & 2 & 89.22 & 89.92 & 89.82 & 89.51 & 88.82 \\
\hline Line.13 & 1 & 169.57 & 170.92 & 170.75 & 170.13 & 168.78 \\
\hline Line.13 & 2 & 169.57 & 170.92 & 170.75 & 170.13 & 168.78 \\
\hline Line.14 & 1 & 257.47 & 259.59 & 259.33 & 258.35 & 256.25 \\
\hline Line.14 & 2 & 257.47 & 259.59 & 259.33 & 258.36 & 256.25 \\
\hline Line.15 & 1 & 171.36 & 172.63 & 172.44 & 171.86 & 170.60 \\
\hline
\end{tabular}




\begin{tabular}{|c|c|c|c|c|c|c|}
\hline \multirow[t]{2}{*}{ Element } & \multirow[t]{2}{*}{ Terminal } & \multicolumn{5}{|c|}{ Faulty Node } \\
\hline & & 744 & 727 & 730 & 709 & 708 \\
\hline Line.15 & 2 & 171.36 & 172.63 & 172.44 & 171.86 & 170.60 \\
\hline Line.16 & 1 & 85.37 & 86.03 & 85.97 & 85.66 & 84.99 \\
\hline Line.16 & 2 & 85.37 & 86.03 & 85.97 & 85.66 & 84.99 \\
\hline Line.17 & 1 & 0.91 & 0.87 & 0.86 & 0.88 & 0.93 \\
\hline Line.17 & 2 & 0.91 & 0.87 & 0.86 & 0.88 & 0.93 \\
\hline Line.18 & 1 & 0.91 & 0.87 & 0.86 & 0.88 & 0.93 \\
\hline Line.18 & 2 & 0.91 & 0.87 & 0.86 & 0.88 & 0.93 \\
\hline Line.19 & 1 & 88.14 & 88.68 & 88.59 & 88.34 & 87.81 \\
\hline Line.19 & 2 & 88.14 & 88.68 & 88.59 & 88.34 & 87.81 \\
\hline Line.110 & 1 & 19.36 & 19.36 & 19.36 & 19.36 & 19.36 \\
\hline Line.110 & 2 & 19.36 & 19.36 & 19.36 & 19.36 & 19.36 \\
\hline Line.111 & 1 & 107.20 & 107.81 & 107.69 & 107.42 & 106.83 \\
\hline Line.111 & 2 & 107.20 & 107.81 & 107.69 & 107.42 & 106.83 \\
\hline Line.112 & 1 & 86.12 & 86.71 & 86.59 & 86.32 & 85.74 \\
\hline Line.112 & 2 & 86.12 & 86.71 & 86.59 & 86.32 & 85.74 \\
\hline Line.113 & 1 & 90.66 & 91.27 & 91.14 & 90.87 & 90.28 \\
\hline Line.113 & 2 & 90.66 & 91.27 & 91.14 & 90.87 & 90.28 \\
\hline Line.114 & 1 & 1.82 & 1.72 & 1.71 & 1.76 & 1.85 \\
\hline Line.114 & 2 & 1.82 & 1.72 & 1.71 & 1.76 & 1.85 \\
\hline Line.115 & 1 & 91.03 & 91.64 & 91.52 & 91.25 & 90.66 \\
\hline Line.115 & 2 & 91.03 & 91.64 & 91.52 & 91.25 & 90.66 \\
\hline Line.116 & 1 & 559.52 & 564.08 & 116.83 & 116.42 & 115.55 \\
\hline Line.116 & 2 & 559.52 & 564.08 & 116.83 & 116.42 & 115.55 \\
\hline Line.117 & 1 & 302.08 & 304.51 & 376.10 & 374.72 & 371.74 \\
\hline Line.117 & 2 & 302.08 & 304.51 & 376.10 & 374.72 & 371.74 \\
\hline Line.118 & 1 & 303.70 & 306.05 & 311.69 & 373.39 & 370.33 \\
\hline Line.118 & 2 & 303.70 & 306.05 & 311.69 & 373.39 & 370.33 \\
\hline Line.119 & 1 & 123.08 & 123.82 & 125.53 & 125.81 & 125.06 \\
\hline Line.119 & 2 & 123.08 & 123.82 & 125.53 & 125.81 & 125.06 \\
\hline Line. 120 & 1 & 180.79 & 182.41 & 186.37 & 187.02 & 495.33 \\
\hline Line. 120 & 2 & 180.79 & 182.41 & 186.37 & 187.02 & 495.33 \\
\hline Line.121 & 1 & 0.94 & 0.89 & 0.75 & 0.72 & 0.70 \\
\hline Line.121 & 2 & 0.94 & 0.89 & 0.75 & 0.72 & 0.70 \\
\hline Line. 122 & 1 & 181.72 & 183.30 & 187.11 & 187.74 & 188.31 \\
\hline Line.122 & 2 & 181.72 & 183.30 & 187.11 & 187.74 & 188.31 \\
\hline Line. 123 & 1 & 558.78 & 118.89 & 117.62 & 117.24 & 116.41 \\
\hline Line.123 & 2 & 558.78 & 118.89 & 117.62 & 117.23 & 116.41 \\
\hline Line.124 & 1 & 121.75 & 121.67 & 120.77 & 120.48 & 119.83 \\
\hline Line.124 & 2 & 121.75 & 121.67 & 120.77 & 120.48 & 119.83 \\
\hline
\end{tabular}




\begin{tabular}{|l|c|c|c|c|c|c|}
\hline Element & Terminal & \multicolumn{5}{|c|}{ Faulty Node } \\
\hline & & $\mathbf{7 4 4}$ & $\mathbf{7 2 7}$ & $\mathbf{7 3 0}$ & $\mathbf{7 0 9}$ & $\mathbf{7 0 8}$ \\
\hline Line.125 & 1 & 2.05 & 2.10 & 2.38 & 2.45 & 2.59 \\
\hline Line.125 & 2 & 2.05 & 2.10 & 2.38 & 2.45 & 2.59 \\
\hline Line.126 & 1 & 191.93 & 193.52 & 197.34 & 197.96 & 198.53 \\
\hline Line.126 & 2 & 191.92 & 193.52 & 197.34 & 197.96 & 198.53 \\
\hline Line.127 & 1 & 84.40 & 85.20 & 87.15 & 87.47 & 87.76 \\
\hline Line.127 & 2 & 84.40 & 85.20 & 87.15 & 87.47 & 87.76 \\
\hline Line.128 & 1 & 101.17 & 102.00 & 103.98 & 104.30 & 104.59 \\
\hline Line.128 & 2 & 101.17 & 101.99 & 103.98 & 104.30 & 104.59 \\
\hline Line.129 & 1 & 104.09 & 104.78 & 106.36 & 106.63 & 106.86 \\
\hline Line.129 & 2 & 104.09 & 104.78 & 106.36 & 106.63 & 106.86 \\
\hline Line.130 & 1 & 106.18 & 106.79 & 108.08 & 108.30 & 108.48 \\
\hline Line.130 & 2 & 106.18 & 106.79 & 108.08 & 108.30 & 108.48 \\
\hline Line.131 & 1 & 2.11 & 2.01 & 1.72 & 1.68 & 1.63 \\
\hline Line.131 & 2 & 2.11 & 2.01 & 1.72 & 1.68 & 1.63 \\
\hline Line.132 & 1 & 108.54 & 109.28 & 111.00 & 111.29 & 111.54 \\
\hline Line.132 & 2 & 108.54 & 109.28 & 111.00 & 111.29 & 111.54 \\
\hline Line.133 & 1 & 110.62 & 111.27 & 112.70 & 112.94 & 113.14 \\
\hline Line.133 & 2 & 110.62 & 111.27 & 112.70 & 112.94 & 113.14 \\
\hline Line.134 & 1 & 2.08 & 1.99 & 1.70 & 1.65 & 1.60 \\
\hline Line.134 & 2 & 2.08 & 1.99 & 1.70 & 1.65 & 1.60 \\
\hline
\end{tabular}

Table 4-28: $I_{r}$ value, zone 2, SLG fault, $100 \%$ penetration

\begin{tabular}{|c|c|c|c|c|c|c|}
\hline Element & Terminal & \multicolumn{5}{|c|}{ Faulty Node } \\
\hline & & $\mathbf{7 4 4}$ & $\mathbf{7 2 7}$ & $\mathbf{7 3 0}$ & $\mathbf{7 0 9}$ & $\mathbf{7 0 8}$ \\
\hline Vsource & 1 & 0.00 & 0.00 & 0.00 & 0.00 & 0.00 \\
\hline Vsource & 2 & 0.00 & 0.00 & 0.00 & 0.00 & 0.00 \\
\hline Subxf & 1 & 0.00 & 0.00 & 0.00 & 0.00 & 0.00 \\
\hline Subxf & 2 & 0.00 & 0.00 & 0.00 & 0.00 & 0.00 \\
\hline Xfm1 & 1 & 0.00 & 0.00 & 0.00 & 0.00 & 0.00 \\
\hline Xfm1 & 2 & 0.00 & 0.00 & 0.00 & 0.00 & 0.00 \\
\hline Line.11 & 1 & 0.13 & 0.13 & 0.13 & 0.13 & 0.12 \\
\hline Line.11 & 2 & 0.13 & 0.13 & 0.13 & 0.13 & 0.12 \\
\hline Line.12 & 1 & 110.72 & 112.64 & 111.81 & 110.82 & 108.93 \\
\hline Line.12 & 2 & 110.72 & 112.64 & 111.81 & 110.82 & 108.93 \\
\hline Line.13 & 1 & 219.54 & 223.36 & 221.70 & 219.73 & 215.98 \\
\hline Line.13 & 2 & 219.54 & 223.36 & 221.70 & 219.73 & 215.98 \\
\hline Line.14 & 1 & 329.97 & 335.70 & 333.21 & 330.25 & 324.61 \\
\hline
\end{tabular}




\begin{tabular}{|c|c|c|c|c|c|c|}
\hline \multirow[t]{2}{*}{ Element } & \multirow[t]{2}{*}{ Terminal } & \multicolumn{5}{|c|}{$\begin{array}{l}\text { Faulty Node } \\
\end{array}$} \\
\hline & & 744 & 727 & 730 & 709 & 708 \\
\hline Line.14 & 2 & 329.97 & 335.70 & 333.21 & 330.25 & 324.61 \\
\hline Line. 15 & 1 & 219.56 & 223.37 & 221.71 & 219.74 & 215.99 \\
\hline Line. 15 & 2 & 219.56 & 223.37 & 221.71 & 219.74 & 215.99 \\
\hline Line.16 & 1 & 110.84 & 112.76 & 111.93 & 110.93 & 109.04 \\
\hline Line.16 & 2 & 110.84 & 112.76 & 111.93 & 110.93 & 109.04 \\
\hline Line.17 & 1 & 0.03 & 0.03 & 0.03 & 0.03 & 0.03 \\
\hline Line.17 & 2 & 0.03 & 0.03 & 0.03 & 0.03 & 0.03 \\
\hline Line.18 & 1 & 0.01 & 0.01 & 0.01 & 0.01 & 0.01 \\
\hline Line.18 & 2 & 0.01 & 0.01 & 0.01 & 0.01 & 0.01 \\
\hline Line.19 & 1 & 110.89 & 112.82 & 111.98 & 110.99 & 109.09 \\
\hline Line.19 & 2 & 110.89 & 112.82 & 111.98 & 110.99 & 109.09 \\
\hline Line. 110 & 1 & 0.00 & 0.00 & 0.00 & 0.00 & 0.00 \\
\hline Line. 110 & 2 & 0.00 & 0.00 & 0.00 & 0.00 & 0.00 \\
\hline Line.111 & 1 & 110.92 & 112.84 & 112.01 & 111.01 & 109.12 \\
\hline Line.111 & 2 & 110.92 & 112.84 & 112.01 & 111.01 & 109.12 \\
\hline Line.112 & 1 & 108.80 & 110.69 & 109.87 & 108.89 & 107.03 \\
\hline Line.112 & 2 & 108.80 & 110.69 & 109.87 & 108.89 & 107.03 \\
\hline Line.113 & 1 & 108.80 & 110.69 & 109.87 & 108.89 & 107.03 \\
\hline Line.113 & 2 & 108.80 & 110.69 & 109.87 & 108.89 & 107.03 \\
\hline Line.114 & 1 & 0.01 & 0.01 & 0.01 & 0.01 & 0.01 \\
\hline Line.114 & 2 & 0.01 & 0.01 & 0.01 & 0.01 & 0.01 \\
\hline Line.115 & 1 & 110.74 & 112.66 & 111.83 & 110.83 & 108.94 \\
\hline Line.115 & 2 & 110.74 & 112.66 & 111.83 & 110.83 & 108.94 \\
\hline Line.116 & 1 & 709.72 & 722.04 & 134.18 & 132.99 & 130.72 \\
\hline Line.116 & 2 & 709.72 & 722.04 & 134.18 & 132.99 & 130.72 \\
\hline Line.117 & 1 & 380.01 & 386.61 & 467.14 & 462.99 & 455.09 \\
\hline Line.117 & 2 & 380.01 & 386.61 & 467.14 & 462.99 & 455.09 \\
\hline Line.118 & 1 & 380.03 & 386.63 & 404.55 & 462.97 & 455.07 \\
\hline Line.118 & 2 & 380.03 & 386.63 & 404.55 & 462.97 & 455.07 \\
\hline Line.119 & 1 & 129.58 & 131.83 & 137.94 & 139.15 & 136.77 \\
\hline Line.119 & 2 & 129.58 & 131.83 & 137.94 & 139.15 & 136.77 \\
\hline Line. 120 & 1 & 250.85 & 255.20 & 267.03 & 269.37 & 591.11 \\
\hline Line. 120 & 2 & 250.85 & 255.20 & 267.03 & 269.37 & 591.11 \\
\hline Line.121 & 1 & 0.01 & 0.01 & 0.01 & 0.01 & 0.01 \\
\hline Line.121 & 2 & 0.01 & 0.01 & 0.01 & 0.01 & 0.01 \\
\hline Line.122 & 1 & 250.86 & 255.22 & 267.05 & 269.39 & 272.45 \\
\hline Line. 122 & 2 & 250.86 & 255.22 & 267.05 & 269.39 & 272.45 \\
\hline Line.123 & 1 & 709.71 & 138.85 & 134.18 & 132.99 & 130.72 \\
\hline Line.123 & 2 & 709.71 & 138.85 & 134.18 & 132.99 & 130.72 \\
\hline
\end{tabular}




\begin{tabular}{|l|c|c|c|c|c|c|}
\hline Element & Terminal & \multicolumn{7}{|c|}{ Faulty Node } \\
\hline & & $\mathbf{7 4 4}$ & $\mathbf{7 2 7}$ & $\mathbf{7 3 0}$ & $\mathbf{7 0 9}$ & $\mathbf{7 0 8}$ \\
\hline Line.124 & 1 & 140.02 & 138.86 & 134.20 & 133.01 & 130.74 \\
\hline Line.124 & 2 & 140.02 & 138.86 & 134.20 & 133.01 & 130.74 \\
\hline Line.125 & 1 & 0.01 & 0.01 & 0.01 & 0.01 & 0.01 \\
\hline Line.125 & 2 & 0.01 & 0.01 & 0.01 & 0.01 & 0.01 \\
\hline Line.126 & 1 & 250.87 & 255.23 & 267.06 & 269.40 & 272.46 \\
\hline Line.126 & 2 & 250.87 & 255.23 & 267.06 & 269.40 & 272.46 \\
\hline Line.127 & 1 & 125.75 & 127.94 & 133.87 & 135.04 & 136.57 \\
\hline Line.127 & 2 & 125.75 & 127.94 & 133.87 & 135.04 & 136.57 \\
\hline Line.128 & 1 & 125.77 & 127.96 & 133.89 & 135.06 & 136.59 \\
\hline Line.128 & 2 & 125.77 & 127.96 & 133.89 & 135.06 & 136.59 \\
\hline Line.129 & 1 & 125.78 & 127.97 & 133.90 & 135.07 & 136.61 \\
\hline Line.129 & 2 & 125.78 & 127.97 & 133.90 & 135.07 & 136.61 \\
\hline Line.130 & 1 & 125.80 & 127.99 & 133.92 & 135.09 & 136.63 \\
\hline Line.130 & 2 & 125.80 & 127.99 & 133.92 & 135.09 & 136.63 \\
\hline Line.131 & 1 & 0.00 & 0.00 & 0.01 & 0.01 & 0.01 \\
\hline Line.131 & 2 & 0.00 & 0.00 & 0.01 & 0.01 & 0.01 \\
\hline Line.132 & 1 & 125.15 & 127.32 & 133.22 & 134.39 & 135.91 \\
\hline Line.132 & 2 & 125.15 & 127.32 & 133.22 & 134.39 & 135.91 \\
\hline Line.133 & 1 & 125.17 & 127.34 & 133.24 & 134.41 & 135.93 \\
\hline Line.133 & 2 & 125.17 & 127.34 & 133.24 & 134.41 & 135.93 \\
\hline Line.134 & 1 & 0.00 & 0.00 & 0.01 & 0.01 & 0.01 \\
\hline Line.134 & 2 & 0.00 & 0.00 & 0.01 & 0.01 & 0.01 \\
\hline
\end{tabular}


Table 4-29: $I_{2}$ value, zone 2, LL fault, $100 \%$ penetration

\begin{tabular}{|c|c|c|c|c|c|c|}
\hline Element & Terminal & \multicolumn{5}{|c|}{ Faulty Node } \\
\hline & & $\mathbf{7 4 4}$ & $\mathbf{7 2 7}$ & $\mathbf{7 3 0}$ & $\mathbf{7 0 9}$ & $\mathbf{7 0 8}$ \\
\hline Vsource & 1 & 0.00 & 0.00 & 0.00 & 0.00 & 0.00 \\
\hline Vsource & 2 & 0.00 & 0.00 & 0.00 & 0.00 & 0.00 \\
\hline Subxf & 1 & 0.00 & 0.00 & 0.00 & 0.00 & 0.00 \\
\hline Subxf & 2 & 0.00 & 0.00 & 0.00 & 0.00 & 0.00 \\
\hline Xfm1 & 1 & 0.00 & 0.00 & 0.00 & 0.00 & 0.00 \\
\hline Xfm1 & 2 & 0.00 & 0.00 & 0.00 & 0.00 & 0.00 \\
\hline Line.11 & 1 & 4.22 & 4.23 & 4.21 & 4.21 & 4.20 \\
\hline Line.11 & 2 & 4.23 & 4.23 & 4.22 & 4.21 & 4.20 \\
\hline Line.12 & 1 & 55.18 & 55.60 & 55.39 & 55.16 & 54.70 \\
\hline Line.12 & 2 & 55.19 & 55.60 & 55.39 & 55.17 & 54.70 \\
\hline Line.13 & 1 & 118.53 & 119.30 & 118.89 & 118.47 & 117.61 \\
\hline Line.13 & 2 & 118.54 & 119.31 & 118.89 & 118.47 & 117.62 \\
\hline Line.14 & 1 & 173.91 & 175.09 & 174.46 & 173.82 & 172.51 \\
\hline Line.14 & 2 & 173.88 & 175.06 & 174.43 & 173.79 & 172.48 \\
\hline Line.15 & 1 & 119.72 & 120.51 & 120.11 & 119.68 & 118.80 \\
\hline Line.15 & 2 & 119.73 & 120.52 & 120.12 & 119.69 & 118.81 \\
\hline Line.16 & 1 & 71.19 & 71.47 & 71.24 & 71.09 & 70.78 \\
\hline Line.16 & 2 & 71.20 & 71.48 & 71.26 & 71.10 & 70.79 \\
\hline Line.17 & 1 & 3.72 & 3.73 & 3.73 & 3.72 & 3.70 \\
\hline Line.17 & 2 & 3.72 & 3.73 & 3.73 & 3.72 & 3.71 \\
\hline Line.18 & 1 & 3.72 & 3.73 & 3.73 & 3.72 & 3.71 \\
\hline Line.18 & 2 & 3.72 & 3.73 & 3.73 & 3.73 & 3.71 \\
\hline Line.19 & 1 & 68.63 & 68.94 & 68.73 & 68.56 & 68.21 \\
\hline Line.19 & 2 & 68.64 & 68.95 & 68.74 & 68.57 & 68.23 \\
\hline Line.110 & 1 & 17.99 & 18.02 & 18.02 & 18.00 & 17.97 \\
\hline Line.110 & 2 & 17.99 & 18.02 & 18.02 & 18.00 & 17.97 \\
\hline Line.111 & 1 & 56.05 & 56.52 & 56.32 & 56.06 & 55.54 \\
\hline Line.111 & 2 & 56.06 & 56.53 & 56.33 & 56.07 & 55.55 \\
\hline Line.112 & 1 & 51.14 & 51.65 & 51.49 & 51.21 & 50.63 \\
\hline Line.112 & 2 & 51.14 & 51.65 & 51.49 & 51.21 & 50.64 \\
\hline Line.113 & 1 & 51.32 & 51.88 & 51.71 & 51.40 & 50.77 \\
\hline Line.113 & 2 & 51.32 & 51.89 & 51.71 & 51.41 & 50.78 \\
\hline Line.114 & 1 & 8.68 & 8.63 & 8.61 & 8.63 & 8.67 \\
\hline Line.114 & 2 & 8.67 & 8.63 & 8.61 & 8.63 & 8.67 \\
\hline Line.115 & 1 & 57.52 & 57.95 & 57.76 & 57.52 & 57.03 \\
\hline Line.115 & 2 & 57.52 & 57.96 & 57.76 & 57.52 & 57.03 \\
\hline Line.116 & 1 & 331.07 & 333.79 & 58.14 & 57.81 & 57.16 \\
\hline
\end{tabular}




\begin{tabular}{|l|c|c|c|c|c|c|}
\hline Element & Terminal & \multicolumn{5}{|c|}{ Faulty Node } \\
\hline & & $\mathbf{7 4 4}$ & $\mathbf{7 2 7}$ & $\mathbf{7 3 0}$ & $\mathbf{7 0 9}$ & $\mathbf{7 0 8}$ \\
\hline Line.116 & 2 & 331.07 & 333.79 & 58.14 & 57.82 & 57.16 \\
\hline Line.117 & 1 & 157.66 & 159.18 & 231.77 & 230.80 & 228.81 \\
\hline Line.117 & 2 & 157.67 & 159.19 & 231.76 & 230.79 & 228.80 \\
\hline Line.118 & 1 & 159.49 & 161.00 & 164.82 & 229.84 & 227.89 \\
\hline Line.118 & 2 & 159.49 & 161.01 & 164.83 & 229.84 & 227.88 \\
\hline Line.119 & 1 & 68.18 & 68.70 & 69.98 & 70.20 & 69.59 \\
\hline Line.119 & 2 & 68.19 & 68.71 & 69.99 & 70.20 & 69.60 \\
\hline Line.120 & 1 & 91.83 & 92.87 & 95.45 & 95.88 & 295.55 \\
\hline Line.120 & 2 & 91.84 & 92.87 & 95.45 & 95.88 & 295.54 \\
\hline Line.121 & 1 & 4.31 & 4.28 & 4.22 & 4.21 & 4.20 \\
\hline Line.121 & 2 & 4.30 & 4.28 & 4.22 & 4.21 & 4.20 \\
\hline Line.122 & 1 & 92.76 & 93.78 & 96.38 & 96.81 & 97.24 \\
\hline Line.122 & 2 & 92.77 & 93.79 & 96.39 & 96.81 & 97.24 \\
\hline Line.123 & 1 & 330.51 & 60.72 & 59.51 & 59.19 & 58.54 \\
\hline Line.123 & 2 & 330.50 & 60.73 & 59.52 & 59.20 & 58.54 \\
\hline Line.124 & 1 & 61.09 & 60.89 & 59.82 & 59.53 & 58.95 \\
\hline Line.124 & 2 & 61.09 & 60.90 & 59.82 & 59.54 & 58.95 \\
\hline Line.125 & 1 & 6.78 & 6.76 & 6.63 & 6.60 & 6.53 \\
\hline Line.125 & 2 & 6.78 & 6.76 & 6.63 & 6.60 & 6.53 \\
\hline Line.126 & 1 & 102.72 & 103.81 & 106.53 & 106.97 & 107.42 \\
\hline Line.126 & 2 & 102.73 & 103.82 & 106.54 & 106.98 & 107.43 \\
\hline Line.127 & 1 & 40.83 & 41.38 & 42.86 & 43.09 & 43.32 \\
\hline Line.127 & 2 & 40.84 & 41.39 & 42.87 & 43.10 & 43.33 \\
\hline Line.128 & 1 & 57.00 & 57.68 & 59.35 & 59.62 & 59.88 \\
\hline Line.128 & 2 & 57.01 & 57.68 & 59.35 & 59.63 & 59.89 \\
\hline Line.129 & 1 & 59.15 & 59.68 & 60.90 & 61.11 & 61.32 \\
\hline Line.129 & 2 & 59.16 & 59.68 & 60.91 & 61.12 & 61.32 \\
\hline Line.130 & 1 & 61.59 & 62.15 & 63.51 & 63.73 & 63.96 \\
\hline Line.130 & 2 & 61.60 & 62.16 & 63.51 & 63.74 & 63.97 \\
\hline Line.131 & 1 & 8.79 & 8.74 & 8.61 & 8.60 & 8.59 \\
\hline Line.131 & 2 & 8.79 & 8.74 & 8.61 & 8.60 & 8.59 \\
\hline Line.132 & 1 & 63.01 & 63.54 & 64.79 & 65.00 & 65.21 \\
\hline Line.132 & 2 & 63.02 & 63.54 & 64.79 & 65.01 & 65.22 \\
\hline Line.133 & 1 & 65.62 & 66.17 & 67.52 & 67.75 & 67.98 \\
\hline Line.133 & 2 & 65.64 & 66.19 & 67.54 & 67.77 & 67.99 \\
\hline Line.134 & 1 & 8.79 & 8.74 & 8.61 & 8.60 & 8.59 \\
\hline Line.134 & 2 & 8.79 & 8.74 & 8.61 & 8.60 & 8.59 \\
\hline
\end{tabular}


Table 4-30: $I_{1}$ value, zone 3, 3 phase fault, $100 \%$ penetration

\begin{tabular}{|c|c|c|c|c|c|c|}
\hline Element & Terminal & \multicolumn{5}{|c|}{ Faulty Node } \\
\hline & & $\mathbf{7 3 4}$ & $\mathbf{7 1 0}$ & $\mathbf{7 3 7}$ & $\mathbf{7 3 8}$ & $\mathbf{7 1 1}$ \\
\hline Vsource & 1 & 0.00 & 0.00 & 0.00 & 0.00 & 0.00 \\
\hline Vsource & 2 & 0.00 & 0.00 & 0.00 & 0.00 & 0.00 \\
\hline Subxf & 1 & 0.00 & 0.00 & 0.00 & 0.00 & 0.00 \\
\hline Subxf & 2 & 0.00 & 0.00 & 0.00 & 0.00 & 0.00 \\
\hline Xfm1 & 1 & 0.00 & 0.00 & 0.00 & 0.00 & 0.00 \\
\hline Xfm1 & 2 & 0.00 & 0.00 & 0.00 & 0.00 & 0.00 \\
\hline Line.11 & 1 & 1.52 & 1.77 & 1.69 & 1.79 & 1.88 \\
\hline Line.11 & 2 & 1.52 & 1.77 & 1.69 & 1.79 & 1.88 \\
\hline Line.12 & 1 & 87.02 & 85.28 & 85.44 & 84.50 & 83.59 \\
\hline Line.12 & 2 & 87.02 & 85.28 & 85.44 & 84.50 & 83.59 \\
\hline Line.13 & 1 & 165.22 & 161.64 & 162.08 & 160.24 & 158.44 \\
\hline Line.13 & 2 & 165.22 & 161.64 & 162.08 & 160.24 & 158.44 \\
\hline Line.14 & 1 & 250.70 & 245.13 & 245.82 & 242.95 & 240.14 \\
\hline Line.14 & 2 & 250.70 & 245.14 & 245.82 & 242.95 & 240.14 \\
\hline Line.15 & 1 & 167.28 & 164.02 & 164.34 & 162.62 & 160.93 \\
\hline Line.15 & 2 & 167.28 & 164.02 & 164.34 & 162.62 & 160.93 \\
\hline Line.16 & 1 & 83.24 & 81.39 & 81.69 & 80.78 & 79.89 \\
\hline Line.16 & 2 & 83.23 & 81.39 & 81.69 & 80.78 & 79.88 \\
\hline Line.17 & 1 & 1.05 & 1.20 & 1.15 & 1.21 & 1.27 \\
\hline Line.17 & 2 & 1.05 & 1.20 & 1.15 & 1.21 & 1.27 \\
\hline Line.18 & 1 & 1.05 & 1.20 & 1.15 & 1.21 & 1.27 \\
\hline Line.18 & 2 & 1.05 & 1.20 & 1.15 & 1.21 & 1.27 \\
\hline Line.19 & 1 & 86.38 & 84.99 & 85.11 & 84.36 & 83.63 \\
\hline Line.19 & 2 & 86.38 & 84.99 & 85.11 & 84.36 & 83.63 \\
\hline Line.110 & 1 & 19.36 & 19.36 & 19.36 & 19.36 & 19.36 \\
\hline Line.110 & 2 & 19.36 & 19.36 & 19.36 & 19.36 & 19.36 \\
\hline Line.111 & 1 & 105.26 & 103.83 & 103.87 & 103.05 & 102.25 \\
\hline Line.111 & 2 & 105.26 & 103.83 & 103.87 & 103.05 & 102.25 \\
\hline Line.112 & 1 & 84.22 & 82.83 & 82.86 & 82.07 & 81.30 \\
\hline Line.112 & 2 & 84.22 & 82.83 & 82.86 & 82.07 & 81.30 \\
\hline Line.113 & 1 & 88.74 & 87.35 & 87.36 & 86.56 & 85.78 \\
\hline Line.113 & 2 & 88.74 & 87.35 & 87.36 & 86.56 & 85.78 \\
\hline Line.114 & 1 & 2.12 & 2.45 & 2.34 & 2.47 & 2.60 \\
\hline Line.114 & 2 & 2.12 & 2.45 & 2.34 & 2.47 & 2.60 \\
\hline Line.115 & 1 & 89.11 & 87.69 & 87.72 & 86.92 & 86.13 \\
\hline Line.115 & 2 & 89.11 & 87.69 & 87.72 & 86.92 & 86.13 \\
\hline Line.116 & 1 & 113.25 & 110.96 & 111.23 & 110.05 & 108.89 \\
\hline
\end{tabular}




\begin{tabular}{|l|c|c|c|c|c|c|}
\hline Element & Terminal & \multicolumn{5}{|c|}{ Faulty Node } \\
\hline & & $\mathbf{7 3 4}$ & $\mathbf{7 1 0}$ & $\mathbf{7 3 7}$ & $\mathbf{7 3 8}$ & $\mathbf{7 1 1}$ \\
\hline Line.116 & 2 & 113.25 & 110.96 & 111.23 & 110.05 & 108.89 \\
\hline Line.117 & 1 & 363.88 & 356.03 & 356.99 & 352.93 & 348.96 \\
\hline Line.117 & 2 & 363.89 & 356.03 & 356.99 & 352.93 & 348.97 \\
\hline Line.118 & 1 & 362.25 & 354.10 & 355.18 & 351.01 & 346.93 \\
\hline Line.118 & 2 & 362.25 & 354.10 & 355.18 & 351.01 & 346.93 \\
\hline Line.119 & 1 & 123.08 & 121.25 & 121.32 & 120.30 & 119.29 \\
\hline Line.119 & 2 & 123.08 & 121.25 & 121.32 & 120.30 & 119.29 \\
\hline Line.120 & 1 & 485.29 & 475.31 & 476.47 & 471.28 & 466.20 \\
\hline Line.120 & 2 & 485.29 & 475.31 & 476.47 & 471.28 & 466.20 \\
\hline Line.121 & 1 & 0.82 & 0.99 & 0.93 & 1.00 & 1.07 \\
\hline Line.121 & 2 & 0.82 & 0.99 & 0.93 & 1.00 & 1.07 \\
\hline Line.122 & 1 & 484.47 & 474.33 & 475.55 & 470.29 & 465.16 \\
\hline Line.122 & 2 & 484.47 & 474.33 & 475.55 & 470.29 & 465.16 \\
\hline Line.123 & 1 & 114.22 & 112.09 & 112.30 & 111.17 & 110.07 \\
\hline Line.123 & 2 & 114.22 & 112.09 & 112.30 & 111.17 & 110.07 \\
\hline Line.124 & 1 & 118.13 & 116.62 & 116.61 & 115.72 & 114.85 \\
\hline Line.124 & 2 & 118.13 & 116.62 & 116.61 & 115.72 & 114.85 \\
\hline Line.125 & 1 & 2.98 & 3.46 & 3.30 & 3.50 & 3.69 \\
\hline Line.125 & 2 & 2.98 & 3.46 & 3.30 & 3.50 & 3.69 \\
\hline Line.126 & 1 & 474.26 & 464.15 & 465.40 & 460.18 & 455.08 \\
\hline Line.126 & 2 & 474.26 & 464.15 & 465.40 & 460.18 & 455.08 \\
\hline Line.127 & 1 & 88.61 & 86.39 & 575.01 & 568.64 & 562.41 \\
\hline Line.127 & 2 & 88.61 & 86.39 & 575.01 & 568.64 & 562.41 \\
\hline Line.128 & 1 & 105.45 & 103.20 & 105.67 & 551.82 & 545.63 \\
\hline Line.128 & 2 & 105.45 & 103.20 & 105.67 & 551.82 & 545.63 \\
\hline Line.129 & 1 & 107.55 & 105.78 & 107.69 & 107.83 & 543.47 \\
\hline Line.129 & 2 & 107.55 & 105.78 & 107.69 & 107.83 & 543.47 \\
\hline Line.130 & 1 & 109.03 & 107.60 & 109.11 & 109.21 & 109.31 \\
\hline Line.130 & 2 & 109.03 & 107.60 & 109.11 & 109.21 & 109.31 \\
\hline Line.131 & 1 & 1.48 & 1.82 & 1.42 & 1.38 & 1.34 \\
\hline Line.131 & 2 & 1.48 & 1.82 & 1.42 & 1.38 & 1.34 \\
\hline Line.132 & 1 & 112.29 & 549.69 & 110.44 & 109.35 & 108.29 \\
\hline Line.132 & 2 & 112.29 & 549.69 & 110.44 & 109.35 & 108.29 \\
\hline Line.133 & 1 & 113.75 & 113.81 & 112.11 & 111.16 & 110.22 \\
\hline Line.133 & 2 & 113.75 & 113.81 & 112.11 & 111.16 & 110.22 \\
\hline Line.134 & 1 & 1.46 & 1.36 & 1.68 & 1.81 & 1.95 \\
\hline Line.134 & 2 & 1.46 & 1.36 & 1.68 & 1.81 & 1.95 \\
\hline
\end{tabular}


Table 4-31: $I_{r}$ value, zone 3, SLG fault, $100 \%$ penetration

\begin{tabular}{|c|c|c|c|c|c|c|}
\hline Element & Terminal & \multicolumn{5}{|c|}{ Faulty Node } \\
\hline & & $\mathbf{7 3 4}$ & $\mathbf{7 1 0}$ & $\mathbf{7 3 7}$ & $\mathbf{7 3 8}$ & $\mathbf{7 1 1}$ \\
\hline Vsource & 1 & 0.00 & 0.00 & 0.00 & 0.00 & 0.00 \\
\hline Vsource & 2 & 0.00 & 0.00 & 0.00 & 0.00 & 0.00 \\
\hline Subxf & 1 & 0.00 & 0.00 & 0.00 & 0.00 & 0.00 \\
\hline Subxf & 2 & 0.00 & 0.00 & 0.00 & 0.00 & 0.00 \\
\hline Xfm1 & 1 & 0.00 & 0.00 & 0.00 & 0.00 & 0.00 \\
\hline Xfm1 & 2 & 0.00 & 0.00 & 0.00 & 0.00 & 0.00 \\
\hline Line.11 & 1 & 0.12 & 0.11 & 0.11 & 0.11 & 0.11 \\
\hline Line.11 & 2 & 0.12 & 0.11 & 0.11 & 0.11 & 0.11 \\
\hline Line.12 & 1 & 103.92 & 99.40 & 99.94 & 97.51 & 95.13 \\
\hline Line.12 & 2 & 103.92 & 99.40 & 99.94 & 97.51 & 95.13 \\
\hline Line.13 & 1 & 206.05 & 197.10 & 198.17 & 193.34 & 188.63 \\
\hline Line.13 & 2 & 206.05 & 197.10 & 198.17 & 193.34 & 188.63 \\
\hline Line.14 & 1 & 309.68 & 296.24 & 297.84 & 290.58 & 283.51 \\
\hline Line.14 & 2 & 309.68 & 296.24 & 297.84 & 290.58 & 283.51 \\
\hline Line.15 & 1 & 206.06 & 197.11 & 198.18 & 193.35 & 188.64 \\
\hline Line.15 & 2 & 206.06 & 197.11 & 198.18 & 193.35 & 188.64 \\
\hline Line.16 & 1 & 104.03 & 99.51 & 100.05 & 97.61 & 95.23 \\
\hline Line.16 & 2 & 104.03 & 99.51 & 100.05 & 97.61 & 95.23 \\
\hline Line.17 & 1 & 0.02 & 0.02 & 0.02 & 0.02 & 0.02 \\
\hline Line.17 & 2 & 0.02 & 0.02 & 0.02 & 0.02 & 0.02 \\
\hline Line.18 & 1 & 0.01 & 0.01 & 0.01 & 0.01 & 0.01 \\
\hline Line.18 & 2 & 0.01 & 0.01 & 0.01 & 0.01 & 0.01 \\
\hline Line.19 & 1 & 104.07 & 99.56 & 100.09 & 97.65 & 95.28 \\
\hline Line.19 & 2 & 104.07 & 99.56 & 100.09 & 97.65 & 95.28 \\
\hline Line.110 & 1 & 0.00 & 0.00 & 0.00 & 0.00 & 0.00 \\
\hline Line.110 & 2 & 0.00 & 0.00 & 0.00 & 0.00 & 0.00 \\
\hline Line.111 & 1 & 104.10 & 99.58 & 100.12 & 97.68 & 95.30 \\
\hline Line.111 & 2 & 104.10 & 99.58 & 100.12 & 97.68 & 95.30 \\
\hline Line.112 & 1 & 102.11 & 97.68 & 98.20 & 95.81 & 93.48 \\
\hline Line.112 & 2 & 102.11 & 97.68 & 98.20 & 95.81 & 93.48 \\
\hline Line.113 & 1 & 102.11 & 97.68 & 98.21 & 95.81 & 93.48 \\
\hline Line.113 & 2 & 102.11 & 97.68 & 98.21 & 95.81 & 93.48 \\
\hline Line.114 & 1 & 0.01 & 0.01 & 0.01 & 0.01 & 0.01 \\
\hline Line.114 & 2 & 0.01 & 0.01 & 0.01 & 0.01 & 0.01 \\
\hline Line.115 & 1 & 103.93 & 99.42 & 99.96 & 97.52 & 95.15 \\
\hline Line.115 & 2 & 103.93 & 99.42 & 99.96 & 97.52 & 95.15 \\
\hline Line.116 & 1 & 124.70 & 119.29 & 119.94 & 117.01 & 114.16 \\
\hline
\end{tabular}




\begin{tabular}{|l|c|c|c|c|c|c|}
\hline Element & Terminal & \multicolumn{5}{|c|}{ Faulty Node } \\
\hline & & $\mathbf{7 3 4}$ & $\mathbf{7 1 0}$ & $\mathbf{7 3 7}$ & $\mathbf{7 3 8}$ & $\mathbf{7 1 1}$ \\
\hline Line.116 & 2 & 124.70 & 119.29 & 119.94 & 117.01 & 114.16 \\
\hline Line.117 & 1 & 434.16 & 415.31 & 417.55 & 407.38 & 397.46 \\
\hline Line.117 & 2 & 434.16 & 415.31 & 417.55 & 407.38 & 397.46 \\
\hline Line.118 & 1 & 434.14 & 415.29 & 417.54 & 407.36 & 397.44 \\
\hline Line.118 & 2 & 434.14 & 415.29 & 417.54 & 407.36 & 397.44 \\
\hline Line.119 & 1 & 130.49 & 124.82 & 125.50 & 122.44 & 119.46 \\
\hline Line.119 & 2 & 130.49 & 124.82 & 125.50 & 122.44 & 119.46 \\
\hline Line.120 & 1 & 563.93 & 539.45 & 542.36 & 529.14 & 516.26 \\
\hline Line.120 & 2 & 563.93 & 539.45 & 542.36 & 529.14 & 516.26 \\
\hline Line.121 & 1 & 0.01 & 0.01 & 0.01 & 0.01 & 0.01 \\
\hline Line.121 & 2 & 0.01 & 0.01 & 0.01 & 0.01 & 0.01 \\
\hline Line.122 & 1 & 563.91 & 539.43 & 542.35 & 529.13 & 516.24 \\
\hline Line.122 & 2 & 563.91 & 539.43 & 542.35 & 529.13 & 516.24 \\
\hline Line.123 & 1 & 124.71 & 119.30 & 119.94 & 117.02 & 114.17 \\
\hline Line.123 & 2 & 124.71 & 119.30 & 119.94 & 117.02 & 114.17 \\
\hline Line.124 & 1 & 124.72 & 119.31 & 119.96 & 117.03 & 114.18 \\
\hline Line.124 & 2 & 124.72 & 119.31 & 119.96 & 117.03 & 114.18 \\
\hline Line.125 & 1 & 0.00 & 0.00 & 0.00 & 0.00 & 0.00 \\
\hline Line.125 & 2 & 0.00 & 0.00 & 0.00 & 0.00 & 0.00 \\
\hline Line.126 & 1 & 563.90 & 539.42 & 542.34 & 529.12 & 516.23 \\
\hline Line.126 & 2 & 563.90 & 539.42 & 542.34 & 529.12 & 516.23 \\
\hline Line.127 & 1 & 141.19 & 135.06 & 676.13 & 659.65 & 643.59 \\
\hline Line.127 & 2 & 141.19 & 135.06 & 676.13 & 659.65 & 643.59 \\
\hline Line.128 & 1 & 141.21 & 135.08 & 144.21 & 659.63 & 643.57 \\
\hline Line.128 & 2 & 141.21 & 135.08 & 144.21 & 659.63 & 643.57 \\
\hline Line.129 & 1 & 141.22 & 135.09 & 144.23 & 146.19 & 643.56 \\
\hline Line.129 & 2 & 141.22 & 135.09 & 144.23 & 146.19 & 643.56 \\
\hline Line.130 & 1 & 141.24 & 135.11 & 144.25 & 146.20 & 148.23 \\
\hline Line.130 & 2 & 141.24 & 135.11 & 144.25 & 146.20 & 148.23 \\
\hline Line.131 & 1 & 0.01 & 0.01 & 0.01 & 0.01 & 0.01 \\
\hline Line.131 & 2 & 0.01 & 0.01 & 0.01 & 0.01 & 0.01 \\
\hline Line.132 & 1 & 140.51 & 672.85 & 135.14 & 131.84 & 128.63 \\
\hline Line.132 & 2 & 140.51 & 672.85 & 135.14 & 131.84 & 128.63 \\
\hline Line.133 & 1 & 140.53 & 143.06 & 135.15 & 131.86 & 128.65 \\
\hline Line.133 & 2 & 140.53 & 143.06 & 135.15 & 131.86 & 128.65 \\
\hline Line.134 & 1 & 0.01 & 0.01 & 0.01 & 0.01 & 0.00 \\
\hline Line.134 & 2 & 0.01 & 0.01 & 0.01 & 0.01 & 0.00 \\
\hline
\end{tabular}


Table 4-32: $I_{2}$ value, zone 3, LL fault, $100 \%$ penetration

\begin{tabular}{|c|c|c|c|c|c|c|}
\hline Element & Terminal & \multicolumn{5}{|c|}{ Faulty Node } \\
\hline & & $\mathbf{7 3 4}$ & $\mathbf{7 1 0}$ & $\mathbf{7 3 7}$ & $\mathbf{7 3 8}$ & $\mathbf{7 1 1}$ \\
\hline Vsource & 1 & 0.00 & 0.00 & 0.00 & 0.00 & 0.00 \\
\hline Vsource & 2 & 0.00 & 0.00 & 0.00 & 0.00 & 0.00 \\
\hline Subxf & 1 & 0.00 & 0.00 & 0.00 & 0.00 & 0.00 \\
\hline Subxf & 2 & 0.00 & 0.00 & 0.00 & 0.00 & 0.00 \\
\hline Xfm1 & 1 & 0.00 & 0.00 & 0.00 & 0.00 & 0.00 \\
\hline Xfm1 & 2 & 0.00 & 0.00 & 0.00 & 0.00 & 0.00 \\
\hline Line.11 & 1 & 4.18 & 4.17 & 4.16 & 4.15 & 4.14 \\
\hline Line.11 & 2 & 4.18 & 4.18 & 4.16 & 4.15 & 4.14 \\
\hline Line.12 & 1 & 53.49 & 52.49 & 52.51 & 51.95 & 51.40 \\
\hline Line.12 & 2 & 53.50 & 52.50 & 52.51 & 51.95 & 51.40 \\
\hline Line.13 & 1 & 115.38 & 113.54 & 113.52 & 112.45 & 111.40 \\
\hline Line.13 & 2 & 115.39 & 113.55 & 113.52 & 112.46 & 111.40 \\
\hline Line.14 & 1 & 169.10 & 166.30 & 166.27 & 164.65 & 163.06 \\
\hline Line.14 & 2 & 169.07 & 166.27 & 166.24 & 164.62 & 163.03 \\
\hline Line.15 & 1 & 116.51 & 114.54 & 114.60 & 113.51 & 112.43 \\
\hline Line.15 & 2 & 116.51 & 114.54 & 114.61 & 113.51 & 112.43 \\
\hline Line.16 & 1 & 69.95 & 69.37 & 69.25 & 68.85 & 68.45 \\
\hline Line.16 & 2 & 69.96 & 69.38 & 69.26 & 68.86 & 68.46 \\
\hline Line.17 & 1 & 3.67 & 3.62 & 3.65 & 3.64 & 3.63 \\
\hline Line.17 & 2 & 3.67 & 3.63 & 3.65 & 3.64 & 3.64 \\
\hline Line.18 & 1 & 3.67 & 3.63 & 3.65 & 3.64 & 3.64 \\
\hline Line.18 & 2 & 3.68 & 3.63 & 3.66 & 3.65 & 3.64 \\
\hline Line.19 & 1 & 67.31 & 66.60 & 66.55 & 66.11 & 65.67 \\
\hline Line.19 & 2 & 67.32 & 66.61 & 66.56 & 66.12 & 65.68 \\
\hline Line.110 & 1 & 17.90 & 17.82 & 17.83 & 17.79 & 17.75 \\
\hline Line.110 & 2 & 17.90 & 17.82 & 17.83 & 17.79 & 17.75 \\
\hline Line.111 & 1 & 54.18 & 52.96 & 53.07 & 52.43 & 51.81 \\
\hline Line.111 & 2 & 54.19 & 52.97 & 53.08 & 52.44 & 51.82 \\
\hline Line.112 & 1 & 49.13 & 47.64 & 47.88 & 47.17 & 46.47 \\
\hline Line.112 & 2 & 49.13 & 47.64 & 47.89 & 47.17 & 46.47 \\
\hline Line.113 & 1 & 49.15 & 47.57 & 47.81 & 47.05 & 46.30 \\
\hline Line.113 & 2 & 49.15 & 47.58 & 47.82 & 47.05 & 46.31 \\
\hline Line.114 & 1 & 8.79 & 8.95 & 8.90 & 8.96 & 9.01 \\
\hline Line.114 & 2 & 8.79 & 8.95 & 8.90 & 8.96 & 9.01 \\
\hline Line.115 & 1 & 55.76 & 54.63 & 54.72 & 54.13 & 53.55 \\
\hline Line.115 & 2 & 55.76 & 54.63 & 54.73 & 54.14 & 53.56 \\
\hline Line.116 & 1 & 55.43 & 53.77 & 54.00 & 53.17 & 52.36 \\
\hline
\end{tabular}




\begin{tabular}{|l|c|c|c|c|c|c|}
\hline Element & Terminal & \multicolumn{5}{|c|}{ Faulty Node } \\
\hline & & $\mathbf{7 3 4}$ & $\mathbf{7 1 0}$ & $\mathbf{7 3 7}$ & $\mathbf{7 3 8}$ & $\mathbf{7 1 1}$ \\
\hline Line.116 & 2 & 55.43 & 53.77 & 54.00 & 53.18 & 52.37 \\
\hline Line.117 & 1 & 223.66 & 219.23 & 219.39 & 216.95 & 214.56 \\
\hline Line.117 & 2 & 223.65 & 219.22 & 219.38 & 216.95 & 214.55 \\
\hline Line.118 & 1 & 222.81 & 218.53 & 218.60 & 216.20 & 213.84 \\
\hline Line.118 & 2 & 222.80 & 218.53 & 218.60 & 216.20 & 213.84 \\
\hline Line.119 & 1 & 68.03 & 66.65 & 66.76 & 66.03 & 65.32 \\
\hline Line.119 & 2 & 68.04 & 66.66 & 66.77 & 66.04 & 65.33 \\
\hline Line.120 & 1 & 288.91 & 283.22 & 283.42 & 280.29 & 277.21 \\
\hline Line.120 & 2 & 288.90 & 283.21 & 283.42 & 280.29 & 277.21 \\
\hline Line.121 & 1 & 4.27 & 4.35 & 4.32 & 4.35 & 4.38 \\
\hline Line.121 & 2 & 4.26 & 4.35 & 4.32 & 4.35 & 4.38 \\
\hline Line.122 & 1 & 288.32 & 282.71 & 282.86 & 279.75 & 276.69 \\
\hline Line.122 & 2 & 288.31 & 282.70 & 282.85 & 279.74 & 276.69 \\
\hline Line.123 & 1 & 56.81 & 55.09 & 55.38 & 54.55 & 53.73 \\
\hline Line.123 & 2 & 56.81 & 55.09 & 55.38 & 54.55 & 53.74 \\
\hline Line.124 & 1 & 57.39 & 55.86 & 56.09 & 55.34 & 54.59 \\
\hline Line.124 & 2 & 57.40 & 55.86 & 56.10 & 55.34 & 54.60 \\
\hline Line.125 & 1 & 6.36 & 6.20 & 6.21 & 6.13 & 6.05 \\
\hline Line.125 & 2 & 6.36 & 6.20 & 6.21 & 6.13 & 6.05 \\
\hline Line.126 & 1 & 278.11 & 272.55 & 272.76 & 269.71 & 266.70 \\
\hline Line.126 & 2 & 278.10 & 272.54 & 272.75 & 269.70 & 266.69 \\
\hline Line.127 & 1 & 44.04 & 42.39 & 336.14 & 332.35 & 328.62 \\
\hline Line.127 & 2 & 44.05 & 42.40 & 336.13 & 332.34 & 328.61 \\
\hline Line.128 & 1 & 60.71 & 58.77 & 61.08 & 315.54 & 311.90 \\
\hline Line.128 & 2 & 60.72 & 58.77 & 61.08 & 315.53 & 311.90 \\
\hline Line.129 & 1 & 61.97 & 60.58 & 62.27 & 62.52 & 310.53 \\
\hline Line.129 & 2 & 61.98 & 60.59 & 62.27 & 62.53 & 310.53 \\
\hline Line.130 & 1 & 64.68 & 63.13 & 65.01 & 65.29 & 65.57 \\
\hline Line.130 & 2 & 64.68 & 63.13 & 65.01 & 65.29 & 65.57 \\
\hline Line.131 & 1 & 8.56 & 8.73 & 8.56 & 8.56 & 8.56 \\
\hline Line.131 & 2 & 8.56 & 8.73 & 8.56 & 8.56 & 8.56 \\
\hline Line.132 & 1 & 65.89 & 313.62 & 64.51 & 63.73 & 62.97 \\
\hline Line.132 & 2 & 65.90 & 313.62 & 64.52 & 63.74 & 62.97 \\
\hline Line.133 & 1 & 68.70 & 69.17 & 67.28 & 66.48 & 65.69 \\
\hline Line.133 & 2 & 68.71 & 69.19 & 67.30 & 66.49 & 65.70 \\
\hline Line.134 & 1 & 8.56 & 8.54 & 8.67 & 8.74 & 8.80 \\
\hline Line.134 & 2 & 8.56 & 8.54 & 8.67 & 8.73 & 8.80 \\
\hline
\end{tabular}




\subsubsection{Short Circuit Analysis 75\% DG Penetration}

Table 4-33: $I_{1}$ value, zone 1,3 phase fault, $75 \%$ penetration

\begin{tabular}{|c|c|c|c|c|c|c|c|c|}
\hline \multirow[t]{2}{*}{ Element } & \multirow[t]{2}{*}{ Terminal } & \multicolumn{7}{|c|}{ Faulty Node } \\
\hline & & 705 & 713 & 704 & 714 & 720 & 706 & 707 \\
\hline Vsource & 1 & 12.99 & 13.15 & 12.84 & 12.77 & 12.35 & 11.99 & 11.58 \\
\hline Vsource & 2 & 12.99 & 13.15 & 12.84 & 12.77 & 12.35 & 11.99 & 11.58 \\
\hline Subxf & 1 & 12.99 & 13.15 & 12.84 & 12.77 & 12.35 & 11.99 & 11.58 \\
\hline Subxf & 2 & 622.22 & 630.01 & 615.27 & 611.76 & 591.92 & 574.43 & 554.93 \\
\hline Xfm1 & 1 & 0.00 & 0.00 & 0.00 & 0.00 & 0.00 & 0.00 & 0.00 \\
\hline Xfm1 & 2 & 0.00 & 0.00 & 0.00 & 0.00 & 0.00 & 0.00 & 0.00 \\
\hline Line.11 & 1 & 563.46 & 570.73 & 557.14 & 553.89 & 535.61 & 519.50 & 501.38 \\
\hline Line.11 & 2 & 563.46 & 570.73 & 557.14 & 553.89 & 535.62 & 519.50 & 501.38 \\
\hline Line. 12 & 1 & 1047.76 & 80.77 & 78.49 & 77.95 & 74.89 & 72.21 & 69.20 \\
\hline Line. 12 & 2 & 1047.76 & 80.77 & 78.49 & 77.95 & 74.89 & 72.21 & 69.20 \\
\hline Line.13 & 1 & 144.13 & 997.52 & 971.43 & 965.14 & 930.18 & 899.33 & 864.14 \\
\hline Line.13 & 2 & 144.13 & 997.52 & 971.43 & 965.14 & 930.18 & 899.33 & 864.14 \\
\hline Line.14 & 1 & 340.71 & 346.43 & 336.23 & 333.73 & 320.13 & 308.11 & 293.97 \\
\hline Line.14 & 2 & 340.70 & 346.42 & 336.22 & 333.73 & 320.12 & 308.10 & 293.96 \\
\hline Line. 15 & 1 & 146.98 & 152.49 & 968.81 & 962.44 & 927.14 & 896.00 & 860.27 \\
\hline Line. 15 & 2 & 146.98 & 152.49 & 968.81 & 962.44 & 927.14 & 896.00 & 860.27 \\
\hline Line.16 & 1 & 68.98 & 72.00 & 72.29 & 71.72 & 1004.37 & 970.76 & 932.49 \\
\hline Line.16 & 2 & 68.98 & 72.00 & 72.29 & 71.72 & 1004.37 & 970.76 & 932.50 \\
\hline Line.17 & 1 & 1.45 & 1.20 & 1.15 & 1.19 & 1.09 & 1040.64 & 1.54 \\
\hline Line.17 & 2 & 1.45 & 1.20 & 1.15 & 1.19 & 1.09 & 1040.64 & 1.54 \\
\hline Line. 18 & 1 & 1.45 & 1.20 & 1.15 & 1.19 & 1.09 & 1.06 & 1.54 \\
\hline Line.18 & 2 & 1.45 & 1.20 & 1.15 & 1.19 & 1.09 & 1.06 & 1.54 \\
\hline Line.19 & 1 & 73.33 & 75.62 & 75.74 & 75.30 & 75.73 & 73.46 & 927.87 \\
\hline Line.19 & 2 & 73.33 & 75.62 & 75.74 & 75.30 & 75.73 & 73.46 & 927.87 \\
\hline Line. 110 & 1 & 19.36 & 19.36 & 19.37 & 19.37 & 19.37 & 19.37 & 19.37 \\
\hline Line.110 & 2 & 19.36 & 19.36 & 19.37 & 19.37 & 19.37 & 19.37 & 19.37 \\
\hline Line.111 & 1 & 92.52 & 94.89 & 95.00 & 94.53 & 94.92 & 92.34 & 94.52 \\
\hline Line.111 & 2 & 92.52 & 94.89 & 95.00 & 94.53 & 94.92 & 92.34 & 94.52 \\
\hline Line.112 & 1 & 78.12 & 80.56 & 80.68 & 1033.60 & 77.35 & 74.85 & 72.30 \\
\hline Line.112 & 2 & 78.12 & 80.56 & 80.68 & 1033.60 & 77.35 & 74.85 & 72.30 \\
\hline Line.113 & 1 & 82.67 & 85.13 & 85.25 & 85.20 & 81.87 & 79.34 & 76.78 \\
\hline Line.113 & 2 & 82.67 & 85.13 & 85.25 & 85.20 & 81.87 & 79.34 & 76.78 \\
\hline Line.114 & 1 & 2.31 & 2.62 & 2.90 & 2.99 & 3.34 & 3.66 & 4.20 \\
\hline Line.114 & 2 & 2.31 & 2.62 & 2.90 & 2.99 & 3.34 & 3.66 & 4.20 \\
\hline Line.115 & 1 & 84.55 & 83.39 & 81.37 & 80.91 & 78.18 & 75.78 & 73.31 \\
\hline
\end{tabular}




\begin{tabular}{|c|c|c|c|c|c|c|c|c|}
\hline \multirow[t]{2}{*}{ Element } & \multirow[t]{2}{*}{ Terminal } & \multicolumn{7}{|c|}{ Faulty Node } \\
\hline & & 705 & 713 & 704 & 714 & 720 & 706 & 707 \\
\hline Line.115 & 2 & 84.55 & 83.39 & 81.37 & 80.91 & 78.18 & 75.78 & 73.31 \\
\hline Line.116 & 1 & 94.27 & 95.81 & 93.13 & 92.47 & 88.91 & 85.76 & 82.00 \\
\hline Line.116 & 2 & 94.27 & 95.81 & 93.13 & 92.47 & 88.91 & 85.76 & 82.00 \\
\hline Line.117 & 1 & 246.55 & 250.72 & 243.20 & 241.37 & 231.32 & 222.45 & 212.09 \\
\hline Line.117 & 2 & 246.55 & 250.72 & 243.20 & 241.37 & 231.31 & 222.45 & 212.09 \\
\hline Line.118 & 1 & 249.32 & 253.27 & 245.97 & 244.22 & 234.44 & 225.82 & 215.94 \\
\hline Line.118 & 2 & 249.32 & 253.27 & 245.97 & 244.22 & 234.44 & 225.82 & 215.94 \\
\hline Line.119 & 1 & 92.86 & 93.88 & 91.71 & 91.22 & 88.26 & 85.67 & 83.05 \\
\hline Line.119 & 2 & 92.86 & 93.88 & 91.71 & 91.22 & 88.26 & 85.67 & 83.05 \\
\hline Line.120 & 1 & 156.50 & 159.42 & 154.33 & 153.07 & 146.31 & 140.33 & 133.08 \\
\hline Line.120 & 2 & 156.50 & 159.42 & 154.33 & 153.07 & 146.31 & 140.33 & 133.08 \\
\hline Line. 121 & 1 & 1.56 & 1.44 & 1.58 & 1.62 & 1.79 & 1.95 & 2.21 \\
\hline Line.121 & 2 & 1.56 & 1.44 & 1.58 & 1.62 & 1.79 & 1.95 & 2.21 \\
\hline Line. 122 & 1 & 158.04 & 160.85 & 155.88 & 154.66 & 148.04 & 142.19 & 135.20 \\
\hline Line. 122 & 2 & 158.04 & 160.85 & 155.88 & 154.66 & 148.04 & 142.19 & 135.20 \\
\hline Line. 123 & 1 & 95.74 & 97.16 & 94.60 & 93.98 & 90.57 & 87.54 & 84.04 \\
\hline Line. 123 & 2 & 95.74 & 97.16 & 94.60 & 93.98 & 90.57 & 87.54 & 84.04 \\
\hline Line. 124 & 1 & 101.58 & 102.51 & 100.44 & 99.98 & 97.14 & 94.67 & 92.18 \\
\hline Line.124 & 2 & 101.58 & 102.51 & 100.44 & 99.98 & 97.14 & 94.66 & 92.18 \\
\hline Line. 125 & 1 & 4.44 & 4.07 & 4.47 & 4.60 & 5.11 & 5.58 & 6.37 \\
\hline Line. 125 & 2 & 4.44 & 4.07 & 4.47 & 4.60 & 5.11 & 5.58 & 6.37 \\
\hline Line. 126 & 1 & 168.19 & 170.99 & 165.96 & 164.73 & 158.00 & 152.06 & 145.10 \\
\hline Line.126 & 2 & 168.19 & 170.99 & 165.95 & 164.73 & 158.00 & 152.06 & 145.10 \\
\hline Line. 127 & 1 & 66.85 & 68.26 & 65.78 & 65.17 & 61.89 & 58.98 & 55.50 \\
\hline Line. 127 & 2 & 66.85 & 68.26 & 65.78 & 65.17 & 61.89 & 58.98 & 55.50 \\
\hline Line. 128 & 1 & 83.60 & 85.00 & 82.44 & 81.82 & 78.39 & 75.36 & 71.94 \\
\hline Line. 128 & 2 & 83.60 & 85.00 & 82.44 & 81.82 & 78.39 & 75.36 & 71.94 \\
\hline Line.129 & 1 & 88.36 & 89.39 & 87.21 & 86.71 & 83.74 & 81.15 & 78.48 \\
\hline Line.129 & 2 & 88.35 & 89.39 & 87.21 & 86.71 & 83.74 & 81.15 & 78.48 \\
\hline Line. 130 & 1 & 91.69 & 92.48 & 90.55 & 90.14 & 87.48 & 85.18 & 83.04 \\
\hline Line. 130 & 2 & 91.68 & 92.48 & 90.55 & 90.14 & 87.48 & 85.18 & 83.04 \\
\hline Line. 131 & 1 & 3.35 & 3.11 & 3.38 & 3.46 & 3.81 & 4.12 & 4.63 \\
\hline Line. 131 & 2 & 3.35 & 3.11 & 3.38 & 3.46 & 3.81 & 4.12 & 4.63 \\
\hline Line. 132 & 1 & 102.94 & 104.22 & 101.78 & 101.20 & 97.90 & 95.00 & 91.77 \\
\hline Line. 132 & 2 & 102.94 & 104.22 & 101.78 & 101.20 & 97.90 & 95.00 & 91.77 \\
\hline Line.133 & 1 & 106.24 & 107.28 & 105.09 & 104.59 & 101.59 & 98.96 & 96.25 \\
\hline Line.133 & 2 & 106.24 & 107.28 & 105.08 & 104.58 & 101.59 & 98.96 & 96.25 \\
\hline Line.134 & 1 & 3.34 & 3.09 & 3.37 & 3.45 & 3.80 & 4.11 & 4.62 \\
\hline Line.134 & 2 & 3.34 & 3.09 & 3.37 & 3.45 & 3.80 & 4.11 & 4.62 \\
\hline
\end{tabular}


Table 4-34: $I_{r}$ value, zone 1, SLG fault, $75 \%$ penetration

\begin{tabular}{|c|c|c|c|c|c|c|c|c|}
\hline \multirow[t]{2}{*}{ Element } & \multirow[t]{2}{*}{ Terminal } & \multicolumn{7}{|c|}{ Faulty Node } \\
\hline & & 705 & 713 & 704 & 714 & 720 & 706 & 707 \\
\hline Vsource & 1 & 0.00 & 0.00 & 0.00 & 0.00 & 0.00 & 0.00 & 0.00 \\
\hline Vsource & 2 & 0.00 & 0.00 & 0.00 & 0.00 & 0.00 & 0.00 & 0.00 \\
\hline Subxf & 1 & 0.00 & 0.00 & 0.00 & 0.00 & 0.00 & 0.00 & 0.00 \\
\hline Subxf & 2 & 0.00 & 0.00 & 0.00 & 0.00 & 0.00 & 0.00 & 0.00 \\
\hline $\mathrm{Xfm} 1$ & 1 & 0.00 & 0.00 & 0.00 & 0.00 & 0.00 & 0.00 & 0.00 \\
\hline $\mathrm{Xfm} 1$ & 2 & 0.00 & 0.00 & 0.00 & 0.00 & 0.00 & 0.00 & 0.00 \\
\hline Line.11 & 1 & 0.18 & 0.18 & 0.18 & 0.18 & 0.17 & 0.16 & 0.15 \\
\hline Line.11 & 2 & 0.18 & 0.18 & 0.18 & 0.18 & 0.17 & 0.16 & 0.15 \\
\hline Line.12 & 1 & 945.93 & 152.69 & 147.13 & 145.87 & 138.17 & 131.41 & 125.46 \\
\hline Line.12 & 2 & 945.93 & 152.69 & 147.13 & 145.87 & 138.17 & 131.41 & 125.46 \\
\hline Line. 13 & 1 & 292.71 & 817.35 & 787.61 & 780.85 & 739.64 & 703.45 & 671.59 \\
\hline Line. 13 & 2 & 292.71 & 817.35 & 787.61 & 780.85 & 739.64 & 703.45 & 671.59 \\
\hline Line.14 & 1 & 653.87 & 665.73 & 641.50 & 635.99 & 602.43 & 572.95 & 547.00 \\
\hline Line.14 & 2 & 653.87 & 665.73 & 641.50 & 635.99 & 602.43 & 572.95 & 547.00 \\
\hline Line. 15 & 1 & 292.72 & 306.87 & 787.59 & 780.83 & 739.63 & 703.43 & 671.58 \\
\hline Line. 15 & 2 & 292.72 & 306.87 & 787.59 & 780.83 & 739.63 & 703.43 & 671.58 \\
\hline Line.16 & 1 & 144.43 & 151.41 & 152.33 & 151.02 & 884.63 & 841.35 & 803.25 \\
\hline Line. 16 & 2 & 144.43 & 151.41 & 152.33 & 151.02 & 884.63 & 841.35 & 803.25 \\
\hline Line. 17 & 1 & 0.04 & 0.04 & 0.04 & 0.04 & 0.04 & 984.58 & 0.04 \\
\hline Line.17 & 2 & 0.04 & 0.04 & 0.04 & 0.04 & 0.04 & 984.58 & 0.04 \\
\hline Line. 18 & 1 & 0.01 & 0.01 & 0.01 & 0.01 & 0.01 & 0.01 & 0.01 \\
\hline Line. 18 & 2 & 0.01 & 0.01 & 0.01 & 0.01 & 0.01 & 0.01 & 0.01 \\
\hline Line. 19 & 1 & 144.50 & 151.49 & 152.41 & 151.10 & 153.28 & 145.78 & 803.18 \\
\hline Line.19 & 2 & 144.50 & 151.49 & 152.41 & 151.10 & 153.28 & 145.78 & 803.18 \\
\hline Line.110 & 1 & 0.00 & 0.00 & 0.00 & 0.00 & 0.00 & 0.00 & 0.00 \\
\hline Line.110 & 2 & 0.00 & 0.00 & 0.00 & 0.00 & 0.00 & 0.00 & 0.00 \\
\hline Line.111 & 1 & 144.54 & 151.53 & 152.45 & 151.14 & 153.32 & 145.82 & 154.56 \\
\hline Line.111 & 2 & 144.54 & 151.53 & 152.45 & 151.14 & 153.32 & 145.82 & 154.56 \\
\hline Line.112 & 1 & 148.39 & 155.56 & 156.50 & 930.69 & 146.97 & 139.78 & 133.45 \\
\hline Line.112 & 2 & 148.39 & 155.56 & 156.50 & 930.69 & 146.97 & 139.78 & 133.45 \\
\hline Line.113 & 1 & 148.39 & 155.56 & 156.50 & 156.48 & 146.97 & 139.78 & 133.45 \\
\hline Line.113 & 2 & 148.39 & 155.56 & 156.50 & 156.48 & 146.97 & 139.78 & 133.45 \\
\hline Line.114 & 1 & 0.01 & 0.01 & 0.01 & 0.01 & 0.01 & 0.01 & 0.01 \\
\hline Line.114 & 2 & 0.01 & 0.01 & 0.01 & 0.01 & 0.01 & 0.01 & 0.01 \\
\hline Line.115 & 1 & 156.34 & 152.71 & 147.15 & 145.89 & 138.19 & 131.43 & 125.48 \\
\hline Line.115 & 2 & 156.34 & 152.71 & 147.15 & 145.89 & 138.19 & 131.43 & 125.48 \\
\hline Line.116 & 1 & 168.15 & 171.19 & 164.97 & 163.55 & 154.92 & 147.34 & 140.67 \\
\hline
\end{tabular}




\begin{tabular}{|c|c|c|c|c|c|c|c|c|}
\hline \multirow[t]{2}{*}{ Element } & \multirow[t]{2}{*}{ Terminal } & \multicolumn{7}{|c|}{ Faulty Node } \\
\hline & & 705 & 713 & 704 & 714 & 720 & 706 & 707 \\
\hline Line.116 & 2 & 168.15 & 171.19 & 164.97 & 163.55 & 154.92 & 147.34 & 140.67 \\
\hline Line.117 & 1 & 486.43 & 495.26 & 477.23 & 473.14 & 448.17 & 426.24 & 406.93 \\
\hline Line.117 & 2 & 486.43 & 495.26 & 477.23 & 473.14 & 448.17 & 426.24 & 406.93 \\
\hline Line.118 & 1 & 486.46 & 495.29 & 477.26 & 473.16 & 448.19 & 426.26 & 406.95 \\
\hline Line.118 & 2 & 486.46 & 495.29 & 477.26 & 473.16 & 448.19 & 426.26 & 406.95 \\
\hline Line.119 & 1 & 164.65 & 167.64 & 161.54 & 160.15 & 151.70 & 144.28 & 137.75 \\
\hline Line.119 & 2 & 164.65 & 167.64 & 161.54 & 160.15 & 151.70 & 144.28 & 137.75 \\
\hline Line. 120 & 1 & 322.23 & 328.08 & 316.14 & 313.42 & 296.88 & 282.35 & 269.56 \\
\hline Line. 120 & 2 & 322.23 & 328.08 & 316.14 & 313.42 & 296.88 & 282.35 & 269.56 \\
\hline Line. 121 & 1 & 0.01 & 0.01 & 0.01 & 0.01 & 0.01 & 0.01 & 0.01 \\
\hline Line. 121 & 2 & 0.01 & 0.01 & 0.01 & 0.01 & 0.01 & 0.01 & 0.01 \\
\hline Line.122 & 1 & 322.26 & 328.10 & 316.16 & 313.45 & 296.90 & 282.37 & 269.58 \\
\hline Line. 122 & 2 & 322.26 & 328.10 & 316.16 & 313.45 & 296.90 & 282.37 & 269.58 \\
\hline Line. 123 & 1 & 168.15 & 171.20 & 164.97 & 163.56 & 154.93 & 147.35 & 140.68 \\
\hline Line.123 & 2 & 168.15 & 171.20 & 164.97 & 163.56 & 154.93 & 147.35 & 140.68 \\
\hline Line.124 & 1 & 168.17 & 171.22 & 164.99 & 163.58 & 154.95 & 147.37 & 140.69 \\
\hline Line.124 & 2 & 168.17 & 171.22 & 164.99 & 163.58 & 154.95 & 147.37 & 140.69 \\
\hline Line. 125 & 1 & 0.01 & 0.01 & 0.01 & 0.01 & 0.01 & 0.01 & 0.01 \\
\hline Line.125 & 2 & 0.01 & 0.01 & 0.01 & 0.01 & 0.01 & 0.01 & 0.01 \\
\hline Line.126 & 1 & 322.27 & 328.12 & 316.17 & 313.46 & 296.92 & 282.39 & 269.60 \\
\hline Line.126 & 2 & 322.27 & 328.12 & 316.17 & 313.46 & 296.92 & 282.39 & 269.60 \\
\hline Line. 127 & 1 & 161.49 & 164.42 & 158.44 & 157.08 & 148.79 & 141.51 & 135.10 \\
\hline Line.127 & 2 & 161.49 & 164.42 & 158.44 & 157.08 & 148.79 & 141.51 & 135.10 \\
\hline Line.128 & 1 & 161.52 & 164.45 & 158.47 & 157.11 & 148.81 & 141.53 & 135.12 \\
\hline Line. 128 & 2 & 161.52 & 164.45 & 158.47 & 157.11 & 148.81 & 141.53 & 135.12 \\
\hline Line.129 & 1 & 161.54 & 164.47 & 158.48 & 157.12 & 148.83 & 141.54 & 135.13 \\
\hline Line.129 & 2 & 161.54 & 164.47 & 158.48 & 157.12 & 148.83 & 141.54 & 135.13 \\
\hline Line.130 & 1 & 161.56 & 164.49 & 158.51 & 157.14 & 148.85 & 141.56 & 135.15 \\
\hline Line. 130 & 2 & 161.56 & 164.49 & 158.51 & 157.14 & 148.85 & 141.56 & 135.15 \\
\hline Line.131 & 1 & 0.01 & 0.01 & 0.01 & 0.01 & 0.01 & 0.01 & 0.01 \\
\hline Line.131 & 2 & 0.01 & 0.01 & 0.01 & 0.01 & 0.01 & 0.01 & 0.01 \\
\hline Line.132 & 1 & 160.81 & 163.73 & 157.77 & 156.41 & 148.16 & 140.91 & 134.53 \\
\hline Line.132 & 2 & 160.81 & 163.73 & 157.77 & 156.41 & 148.16 & 140.91 & 134.53 \\
\hline Line. 133 & 1 & 160.84 & 163.75 & 157.79 & 156.44 & 148.18 & 140.93 & 134.55 \\
\hline Line.133 & 2 & 160.84 & 163.75 & 157.79 & 156.44 & 148.18 & 140.93 & 134.55 \\
\hline Line.134 & 1 & 0.01 & 0.01 & 0.01 & 0.01 & 0.01 & 0.01 & 0.01 \\
\hline Line.134 & 2 & 0.01 & 0.01 & 0.01 & 0.01 & 0.01 & 0.01 & 0.01 \\
\hline
\end{tabular}


Table 4-35: $I_{2}$ value, zone 1, $L L$ fault, $75 \%$ penetration

\begin{tabular}{|c|c|c|c|c|c|c|c|c|}
\hline \multirow[t]{2}{*}{ Element } & \multirow[t]{2}{*}{ Terminal } & \multicolumn{7}{|c|}{ Faulty Node } \\
\hline & & 705 & 713 & 704 & 714 & 720 & 706 & 707 \\
\hline Vsource & 1 & 5.63 & 5.72 & 5.56 & 5.53 & 5.32 & 5.13 & 4.93 \\
\hline Vsource & 2 & 5.63 & 5.72 & 5.56 & 5.53 & 5.32 & 5.13 & 4.93 \\
\hline Subxf & 1 & 5.63 & 5.72 & 5.56 & 5.53 & 5.32 & 5.13 & 4.93 \\
\hline Subxf & 2 & 269.59 & 273.92 & 266.61 & 264.76 & 254.96 & 245.89 & 236.00 \\
\hline $\mathrm{Xfm} 1$ & 1 & 0.00 & 0.00 & 0.00 & 0.00 & 0.00 & 0.00 & 0.00 \\
\hline Xfm1 & 2 & 0.00 & 0.00 & 0.00 & 0.00 & 0.00 & 0.00 & 0.00 \\
\hline Line.11 & 1 & 245.36 & 249.27 & 242.63 & 240.95 & 232.04 & 223.78 & 214.85 \\
\hline Line.11 & 2 & 245.34 & 249.25 & 242.60 & 240.93 & 232.01 & 223.76 & 214.82 \\
\hline Line. 12 & 1 & 574.82 & 58.73 & 57.43 & 57.13 & 55.36 & 53.74 & 52.17 \\
\hline Line. 12 & 2 & 574.81 & 58.73 & 57.44 & 57.13 & 55.37 & 53.75 & 52.18 \\
\hline Line. 13 & 1 & 122.01 & 519.38 & 505.18 & 501.60 & 482.44 & 464.64 & 445.42 \\
\hline Line. 13 & 2 & 122.01 & 519.37 & 505.17 & 501.59 & 482.44 & 464.64 & 445.42 \\
\hline Line.14 & 1 & 207.81 & 211.53 & 205.28 & 203.68 & 195.21 & 187.27 & 178.50 \\
\hline Line.14 & 2 & 207.84 & 211.57 & 205.32 & 203.71 & 195.24 & 187.30 & 178.53 \\
\hline Line. 15 & 1 & 122.99 & 126.45 & 504.01 & 500.47 & 481.35 & 463.62 & 444.75 \\
\hline Line. 15 & 2 & 123.00 & 126.46 & 504.00 & 500.46 & 481.34 & 463.62 & 444.75 \\
\hline Line. 16 & 1 & 72.67 & 73.71 & 73.91 & 73.72 & 532.10 & 512.23 & 490.76 \\
\hline Line.16 & 2 & 72.68 & 73.73 & 73.92 & 73.73 & 532.08 & 512.22 & 490.75 \\
\hline Line. 17 & 1 & 4.07 & 4.16 & 4.20 & 4.18 & 4.25 & 581.10 & 4.15 \\
\hline Line. 17 & 2 & 4.07 & 4.17 & 4.20 & 4.19 & 4.26 & 581.09 & 4.15 \\
\hline Line. 18 & 1 & 4.07 & 4.17 & 4.20 & 4.19 & 4.26 & 4.29 & 4.15 \\
\hline Line. 18 & 2 & 4.08 & 4.17 & 4.21 & 4.19 & 4.26 & 4.29 & 4.16 \\
\hline Line. 19 & 1 & 69.50 & 70.74 & 70.98 & 70.76 & 71.24 & 69.88 & 494.23 \\
\hline Line.19 & 2 & 69.51 & 70.75 & 70.99 & 70.77 & 71.26 & 69.89 & 494.21 \\
\hline Line.110 & 1 & 17.95 & 18.06 & 18.07 & 18.05 & 18.08 & 17.98 & 18.10 \\
\hline Line.110 & 2 & 17.95 & 18.06 & 18.08 & 18.06 & 18.09 & 17.98 & 18.10 \\
\hline Line.111 & 1 & 56.35 & 58.39 & 58.78 & 58.42 & 59.22 & 57.32 & 59.91 \\
\hline Line.111 & 2 & 56.36 & 58.40 & 58.79 & 58.43 & 59.23 & 57.33 & 59.93 \\
\hline Line.112 & 1 & 53.05 & 55.56 & 56.05 & 573.84 & 53.45 & 51.40 & 48.92 \\
\hline Line.112 & 2 & 53.05 & 55.57 & 56.05 & 573.84 & 53.45 & 51.41 & 48.92 \\
\hline Line.113 & 1 & 53.19 & 55.89 & 56.39 & 56.44 & 53.55 & 51.34 & 48.73 \\
\hline Line.113 & 2 & 53.19 & 55.90 & 56.40 & 56.45 & 53.55 & 51.35 & 48.73 \\
\hline Line.114 & 1 & 9.72 & 9.83 & 9.95 & 9.99 & 10.13 & 10.27 & 10.50 \\
\hline Line.114 & 2 & 9.72 & 9.83 & 9.95 & 9.99 & 10.13 & 10.27 & 10.50 \\
\hline Line.115 & 1 & 62.23 & 61.12 & 59.78 & 59.44 & 57.64 & 55.98 & 54.17 \\
\hline Line. 115 & 2 & 62.24 & 61.12 & 59.78 & 59.45 & 57.65 & 55.98 & 54.18 \\
\hline Line.116 & 1 & 55.18 & 56.27 & 54.56 & 54.11 & 51.81 & 49.65 & 47.15 \\
\hline
\end{tabular}




\begin{tabular}{|c|c|c|c|c|c|c|c|c|}
\hline \multirow[t]{2}{*}{ Element } & \multirow[t]{2}{*}{ Terminal } & \multicolumn{7}{|c|}{ Faulty Node } \\
\hline & & 705 & 713 & 704 & 714 & 720 & 706 & 707 \\
\hline Line.116 & 2 & 55.19 & 56.28 & 54.56 & 54.11 & 51.81 & 49.65 & 47.15 \\
\hline Line.117 & 1 & 152.86 & 155.48 & 150.95 & 149.80 & 143.64 & 137.87 & 131.63 \\
\hline Line.117 & 2 & 152.87 & 155.49 & 150.96 & 149.81 & 143.64 & 137.88 & 131.64 \\
\hline Line.118 & 1 & 154.36 & 157.16 & 152.61 & 151.42 & 145.24 & 139.42 & 132.81 \\
\hline Line.118 & 2 & 154.36 & 157.16 & 152.61 & 151.42 & 145.25 & 139.42 & 132.81 \\
\hline Line.119 & 1 & 66.91 & 67.77 & 66.29 & 65.92 & 63.92 & 62.07 & 60.06 \\
\hline Line.119 & 2 & 66.92 & 67.78 & 66.30 & 65.93 & 63.93 & 62.08 & 60.07 \\
\hline Line. 120 & 1 & 88.29 & 90.13 & 87.02 & 86.22 & 82.00 & 78.03 & 73.64 \\
\hline Line. 120 & 2 & 88.29 & 90.14 & 87.03 & 86.22 & 82.00 & 78.03 & 73.64 \\
\hline Line.121 & 1 & 4.95 & 4.88 & 4.94 & 4.96 & 5.03 & 5.09 & 5.21 \\
\hline Line.121 & 2 & 4.95 & 4.88 & 4.94 & 4.96 & 5.03 & 5.09 & 5.21 \\
\hline Line. 122 & 1 & 88.97 & 90.93 & 87.82 & 86.99 & 82.78 & 78.78 & 74.13 \\
\hline Line.122 & 2 & 88.97 & 90.94 & 87.83 & 87.00 & 82.79 & 78.79 & 74.13 \\
\hline Line. 123 & 1 & 56.52 & 57.68 & 55.97 & 55.51 & 53.23 & 51.08 & 48.46 \\
\hline Line.123 & 2 & 56.53 & 57.68 & 55.98 & 55.51 & 53.24 & 51.09 & 48.46 \\
\hline Line.124 & 1 & 57.28 & 58.28 & 56.75 & 56.34 & 54.26 & 52.29 & 49.94 \\
\hline Line.124 & 2 & 57.28 & 58.29 & 56.76 & 56.34 & 54.26 & 52.29 & 49.95 \\
\hline Line. 125 & 1 & 7.06 & 7.17 & 6.98 & 6.94 & 6.68 & 6.44 & 6.18 \\
\hline Line. 125 & 2 & 7.06 & 7.17 & 6.98 & 6.94 & 6.68 & 6.44 & 6.18 \\
\hline Line.126 & 1 & 99.00 & 100.94 & 97.70 & 96.86 & 92.48 & 88.37 & 83.87 \\
\hline Line.126 & 2 & 99.01 & 100.95 & 97.71 & 96.87 & 92.49 & 88.38 & 83.87 \\
\hline Line.127 & 1 & 38.06 & 39.31 & 37.60 & 37.11 & 34.83 & 32.62 & 29.73 \\
\hline Line.127 & 2 & 38.07 & 39.32 & 37.61 & 37.12 & 34.84 & 32.63 & 29.74 \\
\hline Line. 128 & 1 & 54.42 & 55.61 & 53.66 & 53.16 & 50.57 & 48.16 & 45.52 \\
\hline Line.128 & 2 & 54.42 & 55.61 & 53.67 & 53.17 & 50.57 & 48.16 & 45.52 \\
\hline Line.129 & 1 & 57.82 & 58.57 & 57.06 & 56.70 & 54.64 & 52.75 & 50.99 \\
\hline Line.129 & 2 & 57.82 & 58.57 & 57.06 & 56.71 & 54.64 & 52.76 & 50.99 \\
\hline Line.130 & 1 & 60.08 & 61.01 & 59.39 & 58.99 & 56.80 & 54.78 & 52.64 \\
\hline Line.130 & 2 & 60.09 & 61.02 & 59.40 & 58.99 & 56.81 & 54.79 & 52.64 \\
\hline Line.131 & 1 & 10.06 & 9.93 & 10.04 & 10.08 & 10.22 & 10.35 & 10.57 \\
\hline Line. 131 & 2 & 10.05 & 9.93 & 10.04 & 10.08 & 10.22 & 10.35 & 10.57 \\
\hline Line.132 & 1 & 61.86 & 62.65 & 61.14 & 60.78 & 58.73 & 56.84 & 54.97 \\
\hline Line. 132 & 2 & 61.86 & 62.66 & 61.15 & 60.79 & 58.74 & 56.85 & 54.97 \\
\hline Line.133 & 1 & 64.38 & 65.31 & 63.74 & 63.34 & 61.23 & 59.26 & 57.10 \\
\hline Line.133 & 2 & 64.39 & 65.33 & 63.76 & 63.36 & 61.24 & 59.28 & 57.12 \\
\hline Line.134 & 1 & 10.07 & 9.94 & 10.06 & 10.10 & 10.23 & 10.36 & 10.58 \\
\hline Line.134 & 2 & 10.07 & 9.94 & 10.05 & 10.09 & 10.23 & 10.36 & 10.58 \\
\hline
\end{tabular}


Table 4-36: $I_{1}$ value, zone 2,3 phase fault, $75 \%$ penetration

\begin{tabular}{|c|c|c|c|c|c|c|}
\hline Element & Terminal & \multicolumn{5}{|c|}{ Faulty Node } \\
\hline & & $\mathbf{7 4 4}$ & $\mathbf{7 2 7}$ & $\mathbf{7 3 0}$ & $\mathbf{7 0 9}$ & $\mathbf{7 0 8}$ \\
\hline Vsource & 1 & 12.62 & 12.78 & 12.70 & 12.60 & 12.42 \\
\hline Vsource & 2 & 12.62 & 12.78 & 12.70 & 12.60 & 12.42 \\
\hline Subxf & 1 & 12.62 & 12.78 & 12.70 & 12.60 & 12.42 \\
\hline Subxf & 2 & 604.51 & 612.58 & 608.34 & 603.57 & 595.15 \\
\hline Xfm1 & 1 & 0.00 & 0.00 & 0.00 & 0.00 & 0.00 \\
\hline Xfm1 & 2 & 0.00 & 0.00 & 0.00 & 0.00 & 0.00 \\
\hline Line.11 & 1 & 547.23 & 554.67 & 550.81 & 546.41 & 538.64 \\
\hline Line.11 & 2 & 547.23 & 554.68 & 550.81 & 546.41 & 538.64 \\
\hline Line.12 & 1 & 76.85 & 78.09 & 77.45 & 76.72 & 75.42 \\
\hline Line.12 & 2 & 76.84 & 78.09 & 77.45 & 76.72 & 75.42 \\
\hline Line.13 & 1 & 139.04 & 141.44 & 140.27 & 138.84 & 136.32 \\
\hline Line.13 & 2 & 139.04 & 141.44 & 140.27 & 138.84 & 136.32 \\
\hline Line.14 & 1 & 762.67 & 773.78 & 768.07 & 761.49 & 749.90 \\
\hline Line.14 & 2 & 762.67 & 773.78 & 768.07 & 761.50 & 749.90 \\
\hline Line.15 & 1 & 142.04 & 144.32 & 143.15 & 141.80 & 139.42 \\
\hline Line.15 & 2 & 142.04 & 144.31 & 143.15 & 141.80 & 139.42 \\
\hline Line.16 & 1 & 66.63 & 67.79 & 67.27 & 66.58 & 65.36 \\
\hline Line.16 & 2 & 66.63 & 67.79 & 67.27 & 66.58 & 65.36 \\
\hline Line.17 & 1 & 1.53 & 1.46 & 1.47 & 1.51 & 1.59 \\
\hline Line.17 & 2 & 1.53 & 1.46 & 1.47 & 1.51 & 1.59 \\
\hline Line.18 & 1 & 1.53 & 1.46 & 1.47 & 1.51 & 1.59 \\
\hline Line.18 & 2 & 1.53 & 1.46 & 1.47 & 1.51 & 1.59 \\
\hline Line.19 & 1 & 71.16 & 72.13 & 71.62 & 71.04 & 70.01 \\
\hline Line.19 & 2 & 71.15 & 72.13 & 71.62 & 71.04 & 70.01 \\
\hline Line.110 & 1 & 19.36 & 19.36 & 19.36 & 19.36 & 19.36 \\
\hline Line.110 & 2 & 19.36 & 19.36 & 19.36 & 19.36 & 19.36 \\
\hline Line.111 & 1 & 90.06 & 91.13 & 90.53 & 89.90 & 88.79 \\
\hline Line.111 & 2 & 90.06 & 91.13 & 90.52 & 89.90 & 88.79 \\
\hline Line.112 & 1 & 75.61 & 76.69 & 76.07 & 75.43 & 74.30 \\
\hline Line.112 & 2 & 75.61 & 76.69 & 76.07 & 75.43 & 74.30 \\
\hline Line.113 & 1 & 80.13 & 81.23 & 80.59 & 79.95 & 78.80 \\
\hline Line.113 & 2 & 80.13 & 81.23 & 80.59 & 79.95 & 78.80 \\
\hline Line.114 & 1 & 3.08 & 2.92 & 2.95 & 3.05 & 3.21 \\
\hline Line.114 & 2 & 3.08 & 2.92 & 2.95 & 3.05 & 3.21 \\
\hline Line.115 & 1 & 79.88 & 80.98 & 80.37 & 79.72 & 78.57 \\
\hline Line.115 & 2 & 79.88 & 80.98 & 80.36 & 79.72 & 78.57 \\
\hline Line.116 & 1 & 1012.73 & 1028.16 & 96.38 & 95.47 & 93.87 \\
\hline
\end{tabular}




\begin{tabular}{|l|c|c|c|c|c|c|}
\hline Element & Terminal & \multicolumn{5}{|c|}{ Faulty Node } \\
\hline & & $\mathbf{7 4 4}$ & $\mathbf{7 2 7}$ & $\mathbf{7 3 0}$ & $\mathbf{7 0 9}$ & $\mathbf{7 0 8}$ \\
\hline Line.116 & 2 & 1012.73 & 1028.16 & 96.38 & 95.47 & 93.87 \\
\hline Line.117 & 1 & 250.26 & 254.58 & 864.00 & 856.51 & 843.31 \\
\hline Line.117 & 2 & 250.26 & 254.58 & 864.00 & 856.51 & 843.31 \\
\hline Line.118 & 1 & 252.83 & 257.02 & 264.44 & 854.36 & 841.01 \\
\hline Line.118 & 2 & 252.83 & 257.02 & 264.44 & 854.36 & 841.01 \\
\hline Line.119 & 1 & 93.76 & 95.02 & 97.02 & 97.17 & 95.79 \\
\hline Line.119 & 2 & 93.76 & 95.02 & 97.02 & 97.17 & 95.79 \\
\hline Line.120 & 1 & 159.11 & 162.02 & 167.43 & 167.94 & 936.66 \\
\hline Line.120 & 2 & 159.11 & 162.02 & 167.43 & 167.94 & 936.66 \\
\hline Line.121 & 1 & 1.45 & 1.38 & 1.17 & 1.14 & 1.11 \\
\hline Line.121 & 2 & 1.45 & 1.38 & 1.17 & 1.14 & 1.11 \\
\hline Line.122 & 1 & 160.54 & 163.39 & 168.60 & 169.08 & 169.51 \\
\hline Line.122 & 2 & 160.54 & 163.39 & 168.60 & 169.08 & 169.51 \\
\hline Line.123 & 1 & 1011.52 & 100.32 & 97.68 & 96.81 & 95.28 \\
\hline Line.123 & 2 & 1011.52 & 100.32 & 97.68 & 96.81 & 95.28 \\
\hline Line.124 & 1 & 104.86 & 104.88 & 102.92 & 102.23 & 100.98 \\
\hline Line.124 & 2 & 104.86 & 104.88 & 102.92 & 102.23 & 100.98 \\
\hline Line.125 & 1 & 3.37 & 3.44 & 3.97 & 4.11 & 4.35 \\
\hline Line.125 & 2 & 3.37 & 3.44 & 3.97 & 4.11 & 4.35 \\
\hline Line.126 & 1 & 170.68 & 173.56 & 178.79 & 179.27 & 179.70 \\
\hline Line.126 & 2 & 170.68 & 173.56 & 178.79 & 179.27 & 179.69 \\
\hline Line.127 & 1 & 68.09 & 69.52 & 72.12 & 72.36 & 72.58 \\
\hline Line.127 & 2 & 68.09 & 69.51 & 72.12 & 72.36 & 72.58 \\
\hline Line.128 & 1 & 84.82 & 86.29 & 88.92 & 89.16 & 89.36 \\
\hline Line.128 & 2 & 84.82 & 86.29 & 88.92 & 89.16 & 89.36 \\
\hline Line.129 & 1 & 89.32 & 90.58 & 92.61 & 92.78 & 92.89 \\
\hline Line.129 & 2 & 89.32 & 90.57 & 92.61 & 92.78 & 92.89 \\
\hline Line.130 & 1 & 92.42 & 93.54 & 95.17 & 95.29 & 95.32 \\
\hline Line.130 & 2 & 92.42 & 93.54 & 95.17 & 95.28 & 95.32 \\
\hline Line.131 & 1 & 3.12 & 2.98 & 2.56 & 2.51 & 2.44 \\
\hline Line.131 & 2 & 3.12 & 2.98 & 2.56 & 2.51 & 2.44 \\
\hline Line.132 & 1 & 104.08 & 105.48 & 107.90 & 108.11 & 108.28 \\
\hline Line.132 & 2 & 104.08 & 105.48 & 107.90 & 108.11 & 108.28 \\
\hline Line.133 & 1 & 107.16 & 108.43 & 110.45 & 110.60 & 110.70 \\
\hline Line.133 & 2 & 107.16 & 108.43 & 110.44 & 110.60 & 110.70 \\
\hline Line.134 & 1 & 3.11 & 2.96 & 2.55 & 2.50 & 2.43 \\
\hline Line.134 & 2 & 3.11 & 2.96 & 2.55 & 2.50 & 2.43 \\
\hline
\end{tabular}


Table 4-37: Ir value, zone 2, SLG fault, 75\% penetration

\begin{tabular}{|c|c|c|c|c|c|c|}
\hline Element & Terminal & \multicolumn{5}{|c|}{ Faulty Node } \\
\hline & & $\mathbf{7 4 4}$ & $\mathbf{7 2 7}$ & $\mathbf{7 3 0}$ & $\mathbf{7 0 9}$ & $\mathbf{7 0 8}$ \\
\hline Vsource & 1 & 0.00 & 0.00 & 0.00 & 0.00 & 0.00 \\
\hline Vsource & 2 & 0.00 & 0.00 & 0.00 & 0.00 & 0.00 \\
\hline Subxf & 1 & 0.00 & 0.00 & 0.00 & 0.00 & 0.00 \\
\hline Subxf & 2 & 0.00 & 0.00 & 0.00 & 0.00 & 0.00 \\
\hline Xfm1 & 1 & 0.00 & 0.00 & 0.00 & 0.00 & 0.00 \\
\hline Xfm1 & 2 & 0.00 & 0.00 & 0.00 & 0.00 & 0.00 \\
\hline Line.11 & 1 & 0.18 & 0.18 & 0.18 & 0.18 & 0.17 \\
\hline Line.11 & 2 & 0.18 & 0.18 & 0.18 & 0.18 & 0.17 \\
\hline Line.12 & 1 & 146.54 & 149.75 & 148.19 & 146.45 & 143.27 \\
\hline Line.12 & 2 & 146.54 & 149.75 & 148.19 & 146.45 & 143.27 \\
\hline Line.13 & 1 & 286.01 & 292.29 & 289.23 & 285.84 & 279.63 \\
\hline Line.13 & 2 & 286.01 & 292.29 & 289.23 & 285.84 & 279.63 \\
\hline Line.14 & 1 & 432.17 & 441.65 & 437.03 & 431.91 & 422.53 \\
\hline Line.14 & 2 & 432.17 & 441.65 & 437.03 & 431.91 & 422.53 \\
\hline Line.15 & 1 & 286.03 & 292.31 & 289.25 & 285.85 & 279.65 \\
\hline Line.15 & 2 & 286.03 & 292.31 & 289.25 & 285.85 & 279.65 \\
\hline Line.16 & 1 & 141.13 & 144.22 & 142.72 & 141.04 & 137.98 \\
\hline Line.16 & 2 & 141.13 & 144.22 & 142.72 & 141.04 & 137.98 \\
\hline Line.17 & 1 & 0.04 & 0.04 & 0.04 & 0.04 & 0.04 \\
\hline Line.17 & 2 & 0.04 & 0.04 & 0.04 & 0.04 & 0.04 \\
\hline Line.18 & 1 & 0.01 & 0.01 & 0.01 & 0.01 & 0.01 \\
\hline Line.18 & 2 & 0.01 & 0.01 & 0.01 & 0.01 & 0.01 \\
\hline Line.19 & 1 & 141.20 & 144.30 & 142.79 & 141.11 & 138.05 \\
\hline Line.19 & 2 & 141.20 & 144.30 & 142.79 & 141.11 & 138.05 \\
\hline Line.110 & 1 & 0.00 & 0.00 & 0.00 & 0.00 & 0.00 \\
\hline Line.110 & 2 & 0.00 & 0.00 & 0.00 & 0.00 & 0.00 \\
\hline Line.111 & 1 & 141.24 & 144.33 & 142.82 & 141.15 & 138.08 \\
\hline Line.111 & 2 & 141.24 & 144.33 & 142.82 & 141.15 & 138.08 \\
\hline Line.112 & 1 & 144.99 & 148.17 & 146.63 & 144.90 & 141.76 \\
\hline Line.112 & 2 & 144.99 & 148.17 & 146.63 & 144.90 & 141.76 \\
\hline Line.113 & 1 & 145.00 & 148.18 & 146.63 & 144.91 & 141.76 \\
\hline Line.113 & 2 & 145.00 & 148.18 & 146.63 & 144.91 & 141.76 \\
\hline Line.114 & 1 & 0.01 & 0.01 & 0.01 & 0.01 & 0.01 \\
\hline Line.114 & 2 & 0.01 & 0.01 & 0.01 & 0.01 & 0.01 \\
\hline Line.115 & 1 & 146.56 & 149.78 & 148.21 & 146.47 & 143.29 \\
\hline Line.115 & 2 & 146.56 & 149.78 & 148.21 & 146.47 & 143.29 \\
\hline Line.116 & 1 & 918.40 & 938.56 & 170.07 & 168.08 & 164.43 \\
\hline
\end{tabular}




\begin{tabular}{|l|c|c|c|c|c|c|}
\hline Element & Terminal & \multicolumn{5}{|c|}{ Faulty Node } \\
\hline & & $\mathbf{7 4 4}$ & $\mathbf{7 2 7}$ & $\mathbf{7 3 0}$ & $\mathbf{7 0 9}$ & $\mathbf{7 0 8}$ \\
\hline Line.116 & 2 & 918.40 & 938.56 & 170.07 & 168.08 & 164.43 \\
\hline Line.117 & 1 & 486.54 & 497.22 & 606.81 & 599.69 & 586.67 \\
\hline Line.117 & 2 & 486.54 & 497.22 & 606.81 & 599.69 & 586.67 \\
\hline Line.118 & 1 & 486.56 & 497.24 & 516.12 & 599.66 & 586.64 \\
\hline Line.118 & 2 & 486.56 & 497.24 & 516.12 & 599.66 & 586.64 \\
\hline Line.119 & 1 & 164.69 & 168.30 & 174.69 & 175.43 & 171.62 \\
\hline Line.119 & 2 & 164.69 & 168.30 & 174.69 & 175.43 & 171.62 \\
\hline Line.120 & 1 & 322.30 & 329.38 & 341.88 & 343.32 & 757.46 \\
\hline Line.120 & 2 & 322.30 & 329.38 & 341.88 & 343.32 & 757.46 \\
\hline Line.121 & 1 & 0.01 & 0.01 & 0.01 & 0.01 & 0.01 \\
\hline Line.121 & 2 & 0.01 & 0.01 & 0.01 & 0.01 & 0.01 \\
\hline Line.122 & 1 & 322.33 & 329.40 & 341.91 & 343.34 & 344.68 \\
\hline Line.122 & 2 & 322.33 & 329.40 & 341.91 & 343.34 & 344.68 \\
\hline Line.123 & 1 & 918.39 & 176.08 & 170.08 & 168.09 & 164.44 \\
\hline Line.123 & 2 & 918.39 & 176.08 & 170.08 & 168.09 & 164.44 \\
\hline Line.124 & 1 & 176.36 & 176.10 & 170.10 & 168.11 & 164.46 \\
\hline Line.124 & 2 & 176.36 & 176.10 & 170.10 & 168.11 & 164.46 \\
\hline Line.125 & 1 & 0.01 & 0.01 & 0.01 & 0.01 & 0.01 \\
\hline Line.125 & 2 & 0.01 & 0.01 & 0.01 & 0.01 & 0.01 \\
\hline Line.126 & 1 & 322.34 & 329.42 & 341.92 & 343.36 & 344.70 \\
\hline Line.126 & 2 & 322.34 & 329.42 & 341.92 & 343.36 & 344.70 \\
\hline Line.127 & 1 & 161.53 & 165.07 & 171.34 & 172.06 & 172.73 \\
\hline Line.127 & 2 & 161.53 & 165.07 & 171.34 & 172.06 & 172.73 \\
\hline Line.128 & 1 & 161.56 & 165.10 & 171.37 & 172.09 & 172.76 \\
\hline Line.128 & 2 & 161.56 & 165.10 & 171.37 & 172.09 & 172.76 \\
\hline Line.129 & 1 & 161.57 & 165.12 & 171.39 & 172.11 & 172.78 \\
\hline Line.129 & 2 & 161.57 & 165.12 & 171.39 & 172.11 & 172.78 \\
\hline Line.130 & 1 & 161.60 & 165.14 & 171.41 & 172.14 & 172.81 \\
\hline Line.130 & 2 & 161.60 & 165.14 & 171.41 & 172.14 & 172.81 \\
\hline Line.131 & 1 & 0.01 & 0.01 & 0.01 & 0.01 & 0.01 \\
\hline Line.131 & 2 & 0.01 & 0.01 & 0.01 & 0.01 & 0.01 \\
\hline Line.132 & 1 & 160.84 & 164.37 & 170.61 & 171.33 & 172.00 \\
\hline Line.132 & 2 & 160.84 & 164.37 & 170.61 & 171.33 & 172.00 \\
\hline Line.133 & 1 & 160.87 & 164.40 & 170.64 & 171.36 & 172.03 \\
\hline Line.133 & 2 & 160.87 & 164.40 & 170.64 & 171.36 & 172.03 \\
\hline Line.134 & 1 & 0.01 & 0.01 & 0.01 & 0.01 & 0.01 \\
\hline Line.134 & 2 & 0.01 & 0.01 & 0.01 & 0.01 & 0.01 \\
\hline
\end{tabular}


Table 4-38: $I_{2}$ value, zone 2, LL fault, $75 \%$ penetration

\begin{tabular}{|c|c|c|c|c|c|c|}
\hline Element & Terminal & \multicolumn{5}{|c|}{ Faulty Node } \\
\hline & & $\mathbf{7 4 4}$ & $\mathbf{7 2 7}$ & $\mathbf{7 3 0}$ & $\mathbf{7 0 9}$ & $\mathbf{7 0 8}$ \\
\hline Vsource & 1 & 5.42 & 5.51 & 5.46 & 5.40 & 5.30 \\
\hline Vsource & 2 & 5.42 & 5.51 & 5.46 & 5.40 & 5.30 \\
\hline Subxf & 1 & 5.42 & 5.51 & 5.46 & 5.40 & 5.30 \\
\hline Subxf & 2 & 259.85 & 264.04 & 261.39 & 258.76 & 254.13 \\
\hline Xfm1 & 1 & 0.00 & 0.00 & 0.00 & 0.00 & 0.00 \\
\hline Xfm1 & 2 & 0.00 & 0.00 & 0.00 & 0.00 & 0.00 \\
\hline Line.11 & 1 & 236.47 & 240.28 & 237.86 & 235.46 & 231.26 \\
\hline Line.11 & 2 & 236.44 & 240.26 & 237.83 & 235.44 & 231.24 \\
\hline Line.12 & 1 & 56.16 & 56.91 & 56.36 & 55.89 & 55.07 \\
\hline Line.12 & 2 & 56.17 & 56.92 & 56.37 & 55.90 & 55.08 \\
\hline Line.13 & 1 & 118.29 & 119.71 & 118.65 & 117.76 & 116.20 \\
\hline Line.13 & 2 & 118.30 & 119.72 & 118.65 & 117.77 & 116.20 \\
\hline Line.14 & 1 & 410.74 & 416.71 & 412.68 & 408.94 & 402.35 \\
\hline Line.14 & 2 & 410.70 & 416.67 & 412.64 & 408.90 & 402.32 \\
\hline Line.15 & 1 & 119.37 & 120.81 & 119.79 & 118.89 & 117.29 \\
\hline Line.15 & 2 & 119.38 & 120.82 & 119.80 & 118.89 & 117.30 \\
\hline Line.16 & 1 & 71.05 & 71.59 & 71.10 & 70.76 & 70.16 \\
\hline Line.16 & 2 & 71.06 & 71.60 & 71.11 & 70.77 & 70.17 \\
\hline Line.17 & 1 & 4.07 & 4.08 & 4.09 & 4.08 & 4.06 \\
\hline Line.17 & 2 & 4.08 & 4.09 & 4.09 & 4.08 & 4.07 \\
\hline Line.18 & 1 & 4.08 & 4.09 & 4.09 & 4.08 & 4.07 \\
\hline Line.18 & 2 & 4.08 & 4.09 & 4.09 & 4.08 & 4.07 \\
\hline Line.19 & 1 & 67.91 & 68.48 & 68.01 & 67.64 & 67.00 \\
\hline Line.19 & 2 & 67.92 & 68.49 & 68.02 & 67.66 & 67.01 \\
\hline Line.110 & 1 & 17.86 & 17.90 & 17.88 & 17.85 & 17.80 \\
\hline Line.110 & 2 & 17.86 & 17.90 & 17.88 & 17.85 & 17.81 \\
\hline Line.111 & 1 & 54.47 & 55.26 & 54.74 & 54.24 & 53.36 \\
\hline Line.111 & 2 & 54.48 & 55.27 & 54.75 & 54.25 & 53.37 \\
\hline Line.112 & 1 & 51.13 & 52.04 & 51.54 & 50.96 & 49.94 \\
\hline Line.112 & 2 & 51.13 & 52.04 & 51.54 & 50.96 & 49.94 \\
\hline Line.113 & 1 & 51.10 & 52.08 & 51.53 & 50.92 & 49.84 \\
\hline Line.113 & 2 & 51.11 & 52.09 & 51.54 & 50.93 & 49.84 \\
\hline Line.114 & 1 & 10.00 & 9.94 & 9.92 & 9.96 & 10.03 \\
\hline Line.114 & 2 & 10.00 & 9.93 & 9.92 & 9.96 & 10.03 \\
\hline Line.115 & 1 & 58.50 & 59.27 & 58.75 & 58.26 & 57.41 \\
\hline Line.115 & 2 & 58.51 & 59.28 & 58.76 & 58.27 & 57.41 \\
\hline Line.116 & 1 & 565.28 & 574.00 & 56.36 & 55.71 & 54.58 \\
\hline
\end{tabular}




\begin{tabular}{|l|c|c|c|c|c|c|}
\hline Element & Terminal & \multicolumn{5}{|c|}{ Faulty Node } \\
\hline & & $\mathbf{7 4 4}$ & $\mathbf{7 2 7}$ & $\mathbf{7 3 0}$ & $\mathbf{7 0 9}$ & $\mathbf{7 0 8}$ \\
\hline Line.116 & 2 & 565.27 & 574.00 & 56.36 & 55.72 & 54.58 \\
\hline Line.117 & 1 & 154.77 & 157.51 & 468.40 & 464.00 & 456.27 \\
\hline Line.117 & 2 & 154.78 & 157.52 & 468.39 & 463.99 & 456.26 \\
\hline Line.118 & 1 & 156.36 & 159.09 & 164.21 & 463.28 & 455.58 \\
\hline Line.118 & 2 & 156.36 & 159.09 & 164.22 & 463.28 & 455.58 \\
\hline Line.119 & 1 & 67.54 & 68.43 & 70.09 & 70.26 & 69.22 \\
\hline Line.119 & 2 & 67.55 & 68.44 & 70.10 & 70.27 & 69.23 \\
\hline Line.120 & 1 & 89.62 & 91.51 & 94.97 & 95.32 & 522.43 \\
\hline Line.120 & 2 & 89.62 & 91.51 & 94.97 & 95.33 & 522.42 \\
\hline Line.121 & 1 & 4.91 & 4.88 & 4.79 & 4.79 & 4.78 \\
\hline Line.121 & 2 & 4.91 & 4.88 & 4.79 & 4.78 & 4.78 \\
\hline Line.122 & 1 & 90.36 & 92.23 & 95.77 & 96.14 & 96.52 \\
\hline Line.122 & 2 & 90.36 & 92.23 & 95.77 & 96.15 & 96.53 \\
\hline Line.123 & 1 & 564.87 & 59.92 & 57.76 & 57.11 & 55.98 \\
\hline Line.123 & 2 & 564.87 & 59.93 & 57.76 & 57.12 & 55.99 \\
\hline Line.124 & 1 & 60.46 & 60.28 & 58.35 & 57.77 & 56.74 \\
\hline Line.124 & 2 & 60.46 & 60.28 & 58.35 & 57.77 & 56.75 \\
\hline Line.125 & 1 & 7.44 & 7.42 & 7.18 & 7.11 & 6.99 \\
\hline Line.125 & 2 & 7.44 & 7.42 & 7.18 & 7.11 & 6.99 \\
\hline Line.126 & 1 & 100.39 & 102.34 & 105.94 & 106.32 & 106.69 \\
\hline Line.126 & 2 & 100.40 & 102.35 & 105.95 & 106.33 & 106.70 \\
\hline Line.127 & 1 & 38.90 & 39.92 & 42.00 & 42.22 & 42.47 \\
\hline Line.127 & 2 & 38.91 & 39.93 & 42.01 & 42.24 & 42.48 \\
\hline Line.128 & 1 & 55.26 & 56.43 & 58.62 & 58.85 & 59.07 \\
\hline Line.128 & 2 & 55.27 & 56.43 & 58.63 & 58.85 & 59.07 \\
\hline Line.129 & 1 & 58.39 & 59.31 & 60.86 & 61.02 & 61.16 \\
\hline Line.129 & 2 & 58.40 & 59.32 & 60.87 & 61.02 & 61.17 \\
\hline Line.130 & 1 & 60.76 & 61.73 & 63.51 & 63.69 & 63.87 \\
\hline Line.130 & 2 & 60.77 & 61.74 & 63.51 & 63.70 & 63.88 \\
\hline Line.131 & 1 & 9.98 & 9.91 & 9.75 & 9.73 & 9.72 \\
\hline Line.131 & 2 & 9.98 & 9.91 & 9.74 & 9.73 & 9.72 \\
\hline Line.132 & 1 & 62.45 & 63.37 & 64.96 & 65.13 & 65.29 \\
\hline Line.132 & 2 & 62.46 & 63.37 & 64.97 & 65.14 & 65.30 \\
\hline Line.133 & 1 & 65.05 & 66.00 & 67.77 & 67.96 & 68.15 \\
\hline Line.133 & 2 & 65.07 & 66.02 & 67.79 & 67.97 & 68.17 \\
\hline Line.134 & 1 & 9.99 & 9.93 & 9.76 & 9.74 & 9.73 \\
\hline Line.134 & 2 & 9.99 & 9.93 & 9.76 & 9.74 & 9.73 \\
\hline
\end{tabular}


Table 4-39: $I_{1}$ value, zone 3,3 phase fault, $75 \%$ penetration

\begin{tabular}{|c|c|c|c|c|c|c|}
\hline Element & Terminal & \multicolumn{5}{|c|}{ Faulty Node } \\
\hline & & $\mathbf{7 3 4}$ & $\mathbf{7 1 0}$ & $\mathbf{7 3 7}$ & $\mathbf{7 3 8}$ & $\mathbf{7 1 1}$ \\
\hline Vsource & 1 & 11.96 & 11.56 & 11.62 & 11.41 & 11.22 \\
\hline Vsource & 2 & 11.96 & 11.56 & 11.62 & 11.41 & 11.22 \\
\hline Subxf & 1 & 11.96 & 11.56 & 11.62 & 11.41 & 11.22 \\
\hline Subxf & 2 & 573.27 & 553.70 & 556.58 & 546.84 & 537.48 \\
\hline Xfm1 & 1 & 0.00 & 0.00 & 0.00 & 0.00 & 0.00 \\
\hline Xfm1 & 2 & 0.00 & 0.00 & 0.00 & 0.00 & 0.00 \\
\hline Line.11 & 1 & 518.46 & 500.33 & 503.08 & 494.10 & 485.48 \\
\hline Line.11 & 2 & 518.47 & 500.34 & 503.09 & 494.11 & 485.48 \\
\hline Line.12 & 1 & 72.07 & 69.06 & 69.52 & 68.03 & 66.60 \\
\hline Line.12 & 2 & 72.07 & 69.06 & 69.52 & 68.03 & 66.60 \\
\hline Line.13 & 1 & 129.78 & 123.68 & 124.80 & 121.88 & 119.08 \\
\hline Line.13 & 2 & 129.78 & 123.68 & 124.80 & 121.88 & 119.07 \\
\hline Line.14 & 1 & 719.79 & 692.58 & 696.85 & 683.46 & 670.59 \\
\hline Line.14 & 2 & 719.80 & 692.59 & 696.86 & 683.47 & 670.59 \\
\hline Line.15 & 1 & 133.24 & 127.58 & 128.51 & 125.75 & 123.09 \\
\hline Line.15 & 2 & 133.24 & 127.58 & 128.51 & 125.75 & 123.09 \\
\hline Line.16 & 1 & 62.20 & 59.10 & 59.78 & 58.36 & 57.00 \\
\hline Line.16 & 2 & 62.19 & 59.10 & 59.77 & 58.36 & 56.99 \\
\hline Line.17 & 1 & 1.79 & 2.02 & 1.94 & 2.03 & 2.11 \\
\hline Line.17 & 2 & 1.79 & 2.02 & 1.94 & 2.03 & 2.11 \\
\hline Line.18 & 1 & 1.79 & 2.02 & 1.94 & 2.03 & 2.11 \\
\hline Line.18 & 2 & 1.79 & 2.02 & 1.94 & 2.03 & 2.11 \\
\hline Line.19 & 1 & 67.33 & 64.87 & 65.27 & 64.06 & 62.89 \\
\hline Line.19 & 2 & 67.33 & 64.87 & 65.26 & 64.05 & 62.89 \\
\hline Line.110 & 1 & 19.36 & 19.36 & 19.36 & 19.36 & 19.36 \\
\hline Line.110 & 2 & 19.36 & 19.36 & 19.36 & 19.36 & 19.36 \\
\hline Line.111 & 1 & 85.90 & 83.41 & 83.68 & 82.39 & 81.15 \\
\hline Line.111 & 2 & 85.90 & 83.41 & 83.68 & 82.39 & 81.15 \\
\hline Line.112 & 1 & 71.37 & 68.86 & 69.13 & 67.83 & 66.58 \\
\hline Line.112 & 2 & 71.37 & 68.86 & 69.13 & 67.83 & 66.58 \\
\hline Line.113 & 1 & 75.84 & 73.34 & 73.59 & 72.28 & 71.02 \\
\hline Line.113 & 2 & 75.84 & 73.34 & 73.59 & 72.28 & 71.02 \\
\hline Line.114 & 1 & 3.63 & 4.11 & 3.95 & 4.13 & 4.32 \\
\hline Line.114 & 2 & 3.63 & 4.10 & 3.95 & 4.13 & 4.32 \\
\hline Line.115 & 1 & 75.60 & 73.05 & 73.33 & 72.01 & 70.74 \\
\hline Line.115 & 2 & 75.60 & 73.05 & 73.33 & 72.01 & 70.74 \\
\hline Line.116 & 1 & 89.72 & 85.88 & 86.57 & 84.73 & 82.96 \\
\hline
\end{tabular}




\begin{tabular}{|l|c|c|c|c|c|c|}
\hline Element & Terminal & \multicolumn{5}{|c|}{ Faulty Node } \\
\hline & & $\mathbf{7 3 4}$ & $\mathbf{7 1 0}$ & $\mathbf{7 3 7}$ & $\mathbf{7 3 8}$ & $\mathbf{7 1 1}$ \\
\hline Line.116 & 2 & 89.72 & 85.87 & 86.57 & 84.73 & 82.96 \\
\hline Line.117 & 1 & 809.03 & 777.97 & 782.93 & 767.68 & 753.03 \\
\hline Line.117 & 2 & 809.04 & 777.97 & 782.93 & 767.69 & 753.04 \\
\hline Line.118 & 1 & 806.38 & 774.88 & 780.01 & 764.61 & 749.81 \\
\hline Line.118 & 2 & 806.38 & 774.88 & 780.01 & 764.61 & 749.81 \\
\hline Line.119 & 1 & 92.21 & 89.16 & 89.47 & 87.89 & 86.36 \\
\hline Line.119 & 2 & 92.21 & 89.16 & 89.47 & 87.88 & 86.36 \\
\hline Line.120 & 1 & 898.50 & 863.99 & 869.43 & 852.45 & 836.14 \\
\hline Line.120 & 2 & 898.50 & 863.99 & 869.43 & 852.45 & 836.14 \\
\hline Line.121 & 1 & 1.32 & 1.57 & 1.48 & 1.58 & 1.67 \\
\hline Line.121 & 2 & 1.32 & 1.57 & 1.48 & 1.58 & 1.67 \\
\hline Line.122 & 1 & 897.19 & 862.44 & 867.96 & 850.90 & 834.50 \\
\hline Line.122 & 2 & 897.19 & 862.44 & 867.97 & 850.90 & 834.50 \\
\hline Line.123 & 1 & 91.30 & 87.68 & 88.28 & 86.51 & 84.82 \\
\hline Line.123 & 2 & 91.30 & 87.68 & 88.28 & 86.51 & 84.82 \\
\hline Line.124 & 1 & 97.76 & 95.03 & 95.29 & 93.84 & 92.45 \\
\hline Line.124 & 2 & 97.76 & 95.03 & 95.28 & 93.84 & 92.45 \\
\hline Line.125 & 1 & 4.98 & 5.70 & 5.46 & 5.74 & 6.00 \\
\hline Line.125 & 2 & 4.98 & 5.70 & 5.46 & 5.74 & 6.00 \\
\hline Line.126 & 1 & 886.98 & 852.27 & 857.82 & 840.79 & 824.43 \\
\hline Line.126 & 2 & 886.98 & 852.27 & 857.82 & 840.79 & 824.43 \\
\hline Line.127 & 1 & 73.27 & 69.38 & 961.35 & 942.36 & 924.11 \\
\hline Line.127 & 2 & 73.27 & 69.37 & 961.36 & 942.36 & 924.11 \\
\hline Line.128 & 1 & 90.02 & 85.96 & 90.17 & 925.55 & 907.35 \\
\hline Line.128 & 2 & 90.02 & 85.96 & 90.17 & 925.55 & 907.35 \\
\hline Line.129 & 1 & 93.29 & 89.96 & 93.30 & 93.37 & 904.00 \\
\hline Line.129 & 2 & 93.29 & 89.96 & 93.30 & 93.37 & 904.00 \\
\hline Line.130 & 1 & 95.52 & 92.69 & 95.43 & 95.43 & 95.45 \\
\hline Line.130 & 2 & 95.52 & 92.69 & 95.42 & 95.43 & 95.45 \\
\hline Line.131 & 1 & 2.24 & 2.75 & 2.13 & 2.07 & 2.01 \\
\hline Line.131 & 2 & 2.24 & 2.75 & 2.13 & 2.07 & 2.01 \\
\hline Line.132 & 1 & 108.84 & 919.73 & 105.58 & 103.68 & 101.85 \\
\hline Line.132 & 2 & 108.84 & 919.73 & 105.58 & 103.68 & 101.84 \\
\hline Line.133 & 1 & 111.06 & 110.89 & 108.09 & 106.35 & 104.69 \\
\hline Line.133 & 2 & 111.06 & 110.89 & 108.08 & 106.35 & 104.68 \\
\hline Line.134 & 1 & 2.23 & 2.08 & 2.55 & 2.76 & 2.95 \\
\hline Line.134 & 2 & 2.23 & 2.08 & 2.55 & 2.76 & 2.95 \\
\hline
\end{tabular}


Table 4-40: $I_{r}$ value, zone 3, SLG fault, $75 \%$ penetration

\begin{tabular}{|c|c|c|c|c|c|c|}
\hline Element & Terminal & \multicolumn{5}{|c|}{ Faulty Node } \\
\hline & & $\mathbf{7 3 4}$ & $\mathbf{7 1 0}$ & $\mathbf{7 3 7}$ & $\mathbf{7 3 8}$ & $\mathbf{7 1 1}$ \\
\hline Vsource & 1 & 0.00 & 0.00 & 0.00 & 0.00 & 0.00 \\
\hline Vsource & 2 & 0.00 & 0.00 & 0.00 & 0.00 & 0.00 \\
\hline Subxf & 1 & 0.00 & 0.00 & 0.00 & 0.00 & 0.00 \\
\hline Subxf & 2 & 0.00 & 0.00 & 0.00 & 0.00 & 0.00 \\
\hline Xfm1 & 1 & 0.00 & 0.00 & 0.00 & 0.00 & 0.00 \\
\hline Xfm1 & 2 & 0.00 & 0.00 & 0.00 & 0.00 & 0.00 \\
\hline Line.11 & 1 & 0.16 & 0.15 & 0.16 & 0.15 & 0.15 \\
\hline Line.11 & 2 & 0.16 & 0.15 & 0.16 & 0.15 & 0.15 \\
\hline Line.12 & 1 & 134.99 & 127.80 & 128.62 & 124.81 & 121.16 \\
\hline Line.12 & 2 & 134.99 & 127.80 & 128.62 & 124.81 & 121.16 \\
\hline Line.13 & 1 & 263.47 & 249.44 & 251.03 & 243.61 & 236.47 \\
\hline Line.13 & 2 & 263.47 & 249.44 & 251.03 & 243.61 & 236.47 \\
\hline Line.14 & 1 & 398.11 & 376.92 & 379.32 & 368.10 & 357.32 \\
\hline Line.14 & 2 & 398.11 & 376.92 & 379.32 & 368.10 & 357.32 \\
\hline Line.15 & 1 & 263.49 & 249.46 & 251.05 & 243.62 & 236.49 \\
\hline Line.15 & 2 & 263.49 & 249.46 & 251.05 & 243.62 & 236.49 \\
\hline Line.16 & 1 & 130.01 & 123.08 & 123.87 & 120.20 & 116.68 \\
\hline Line.16 & 2 & 130.01 & 123.08 & 123.87 & 120.20 & 116.68 \\
\hline Line.17 & 1 & 0.03 & 0.03 & 0.03 & 0.03 & 0.03 \\
\hline Line.17 & 2 & 0.03 & 0.03 & 0.03 & 0.03 & 0.03 \\
\hline Line.18 & 1 & 0.01 & 0.01 & 0.01 & 0.01 & 0.01 \\
\hline Line.18 & 2 & 0.01 & 0.01 & 0.01 & 0.01 & 0.01 \\
\hline Line.19 & 1 & 130.07 & 123.15 & 123.93 & 120.26 & 116.74 \\
\hline Line.19 & 2 & 130.07 & 123.15 & 123.93 & 120.26 & 116.74 \\
\hline Line.110 & 1 & 0.00 & 0.00 & 0.00 & 0.00 & 0.00 \\
\hline Line.110 & 2 & 0.00 & 0.00 & 0.00 & 0.00 & 0.00 \\
\hline Line.111 & 1 & 130.10 & 123.18 & 123.96 & 120.29 & 116.77 \\
\hline Line.111 & 2 & 130.10 & 123.18 & 123.96 & 120.29 & 116.77 \\
\hline Line.112 & 1 & 133.57 & 126.46 & 127.26 & 123.50 & 119.88 \\
\hline Line.112 & 2 & 133.57 & 126.46 & 127.26 & 123.50 & 119.88 \\
\hline Line.113 & 1 & 133.57 & 126.46 & 127.26 & 123.50 & 119.88 \\
\hline Line.113 & 2 & 133.57 & 126.46 & 127.26 & 123.50 & 119.88 \\
\hline Line.114 & 1 & 0.01 & 0.01 & 0.01 & 0.01 & 0.01 \\
\hline Line.114 & 2 & 0.01 & 0.01 & 0.01 & 0.01 & 0.01 \\
\hline Line.115 & 1 & 135.01 & 127.82 & 128.64 & 124.83 & 121.18 \\
\hline Line.115 & 2 & 135.01 & 127.82 & 128.64 & 124.83 & 121.18 \\
\hline Line.116 & 1 & 154.93 & 146.68 & 147.62 & 143.25 & 139.05 \\
\hline
\end{tabular}




\begin{tabular}{|l|c|c|c|c|c|c|}
\hline Element & Terminal & \multicolumn{5}{|c|}{ Faulty Node } \\
\hline & & $\mathbf{7 3 4}$ & $\mathbf{7 1 0}$ & $\mathbf{7 3 7}$ & $\mathbf{7 3 8}$ & $\mathbf{7 1 1}$ \\
\hline Line.116 & 2 & 154.93 & 146.68 & 147.62 & 143.25 & 139.05 \\
\hline Line.117 & 1 & 552.77 & 523.34 & 526.67 & 511.09 & 496.13 \\
\hline Line.117 & 2 & 552.77 & 523.34 & 526.67 & 511.09 & 496.13 \\
\hline Line.118 & 1 & 552.75 & 523.32 & 526.65 & 511.07 & 496.10 \\
\hline Line.118 & 2 & 552.75 & 523.32 & 526.65 & 511.07 & 496.10 \\
\hline Line.119 & 1 & 161.70 & 153.09 & 154.07 & 149.51 & 145.13 \\
\hline Line.119 & 2 & 161.70 & 153.09 & 154.07 & 149.51 & 145.13 \\
\hline Line.120 & 1 & 713.70 & 675.70 & 680.00 & 659.88 & 640.56 \\
\hline Line.120 & 2 & 713.70 & 675.70 & 680.00 & 659.88 & 640.56 \\
\hline Line.121 & 1 & 0.01 & 0.01 & 0.01 & 0.01 & 0.01 \\
\hline Line.121 & 2 & 0.01 & 0.01 & 0.01 & 0.01 & 0.01 \\
\hline Line.122 & 1 & 713.67 & 675.67 & 679.98 & 659.86 & 640.54 \\
\hline Line.122 & 2 & 713.67 & 675.67 & 679.98 & 659.86 & 640.54 \\
\hline Line.123 & 1 & 154.94 & 146.69 & 147.62 & 143.26 & 139.06 \\
\hline Line.123 & 2 & 154.94 & 146.69 & 147.62 & 143.26 & 139.06 \\
\hline Line.124 & 1 & 154.96 & 146.71 & 147.64 & 143.27 & 139.08 \\
\hline Line.124 & 2 & 154.96 & 146.71 & 147.64 & 143.27 & 139.08 \\
\hline Line.125 & 1 & 0.01 & 0.01 & 0.01 & 0.01 & 0.01 \\
\hline Line.125 & 2 & 0.01 & 0.01 & 0.01 & 0.01 & 0.01 \\
\hline Line.126 & 1 & 713.66 & 675.66 & 679.96 & 659.85 & 640.52 \\
\hline Line.126 & 2 & 713.66 & 675.66 & 679.96 & 659.85 & 640.52 \\
\hline Line.127 & 1 & 175.05 & 165.72 & 844.57 & 819.59 & 795.59 \\
\hline Line.127 & 2 & 175.05 & 165.72 & 844.57 & 819.59 & 795.59 \\
\hline Line.128 & 1 & 175.08 & 165.75 & 176.15 & 819.56 & 795.56 \\
\hline Line.128 & 2 & 175.08 & 165.75 & 176.15 & 819.56 & 795.56 \\
\hline Line.129 & 1 & 175.09 & 165.77 & 176.17 & 177.00 & 795.54 \\
\hline Line.129 & 2 & 175.09 & 165.77 & 176.17 & 177.00 & 795.54 \\
\hline Line.130 & 1 & 175.12 & 165.79 & 176.20 & 177.03 & 177.97 \\
\hline Line.130 & 2 & 175.12 & 165.79 & 176.20 & 177.03 & 177.97 \\
\hline Line.131 & 1 & 0.01 & 0.01 & 0.01 & 0.01 & 0.01 \\
\hline Line.131 & 2 & 0.01 & 0.01 & 0.01 & 0.01 & 0.01 \\
\hline Line.132 & 1 & 174.30 & 839.62 & 166.07 & 161.16 & 156.44 \\
\hline Line.132 & 2 & 174.30 & 839.62 & 166.07 & 161.16 & 156.44 \\
\hline Line.133 & 1 & 174.33 & 174.62 & 166.10 & 161.18 & 156.46 \\
\hline Line.133 & 2 & 174.33 & 174.62 & 166.10 & 161.18 & 156.46 \\
\hline Line.134 & 1 & 0.01 & 0.01 & 0.01 & 0.01 & 0.01 \\
\hline Line.134 & 2 & 0.01 & 0.01 & 0.01 & 0.01 & 0.01 \\
\hline
\end{tabular}


Table 4-41: $I_{2}$ value, zone 3, LL fault, $75 \%$ penetration

\begin{tabular}{|c|c|c|c|c|c|c|}
\hline Element & Terminal & \multicolumn{5}{|c|}{ Faulty Node } \\
\hline & & $\mathbf{7 3 4}$ & $\mathbf{7 1 0}$ & $\mathbf{7 3 7}$ & $\mathbf{7 3 8}$ & $\mathbf{7 1 1}$ \\
\hline Vsource & 1 & 5.06 & 4.84 & 4.87 & 4.76 & 4.66 \\
\hline Vsource & 2 & 5.06 & 4.84 & 4.87 & 4.76 & 4.66 \\
\hline Subxf & 1 & 5.06 & 4.84 & 4.87 & 4.76 & 4.66 \\
\hline Subxf & 2 & 242.23 & 231.87 & 233.37 & 228.28 & 223.39 \\
\hline Xfm1 & 1 & 0.00 & 0.00 & 0.00 & 0.00 & 0.00 \\
\hline Xfm1 & 2 & 0.00 & 0.00 & 0.00 & 0.00 & 0.00 \\
\hline Line.11 & 1 & 220.45 & 211.06 & 212.39 & 207.77 & 203.32 \\
\hline Line.11 & 2 & 220.42 & 211.04 & 212.37 & 207.74 & 203.30 \\
\hline Line.12 & 1 & 52.96 & 51.24 & 51.38 & 50.48 & 49.61 \\
\hline Line.12 & 2 & 52.96 & 51.24 & 51.38 & 50.48 & 49.61 \\
\hline Line.13 & 1 & 112.11 & 108.75 & 109.00 & 107.21 & 105.46 \\
\hline Line.13 & 2 & 112.11 & 108.75 & 109.01 & 107.21 & 105.46 \\
\hline Line.14 & 1 & 385.36 & 370.92 & 372.64 & 365.33 & 358.27 \\
\hline Line.14 & 2 & 385.32 & 370.89 & 372.61 & 365.30 & 358.24 \\
\hline Line.15 & 1 & 113.12 & 109.58 & 109.97 & 108.14 & 106.37 \\
\hline Line.15 & 2 & 113.13 & 109.59 & 109.98 & 108.15 & 106.37 \\
\hline Line.16 & 1 & 68.55 & 67.31 & 67.30 & 66.57 & 65.85 \\
\hline Line.16 & 2 & 68.56 & 67.32 & 67.31 & 66.58 & 65.86 \\
\hline Line.17 & 1 & 4.03 & 4.00 & 4.02 & 4.02 & 4.02 \\
\hline Line.17 & 2 & 4.04 & 4.00 & 4.03 & 4.03 & 4.03 \\
\hline Line.18 & 1 & 4.04 & 4.00 & 4.03 & 4.03 & 4.03 \\
\hline Line.18 & 2 & 4.04 & 4.01 & 4.03 & 4.03 & 4.03 \\
\hline Line.19 & 1 & 65.30 & 63.92 & 64.00 & 63.23 & 62.48 \\
\hline Line.19 & 2 & 65.31 & 63.93 & 64.01 & 63.24 & 62.49 \\
\hline Line.110 & 1 & 17.68 & 17.55 & 17.58 & 17.52 & 17.46 \\
\hline Line.110 & 2 & 17.68 & 17.55 & 17.58 & 17.52 & 17.46 \\
\hline Line.111 & 1 & 51.11 & 49.16 & 49.43 & 48.46 & 47.54 \\
\hline Line.111 & 2 & 51.12 & 49.17 & 49.44 & 48.47 & 47.54 \\
\hline Line.112 & 1 & 47.32 & 44.88 & 45.36 & 44.24 & 43.15 \\
\hline Line.112 & 2 & 47.32 & 44.88 & 45.37 & 44.24 & 43.15 \\
\hline Line.113 & 1 & 47.06 & 44.51 & 45.00 & 43.82 & 42.68 \\
\hline Line.113 & 2 & 47.07 & 44.52 & 45.01 & 43.83 & 42.69 \\
\hline Line.114 & 1 & 10.19 & 10.39 & 10.31 & 10.38 & 10.44 \\
\hline Line.114 & 2 & 10.19 & 10.39 & 10.31 & 10.38 & 10.44 \\
\hline Line.115 & 1 & 55.20 & 53.29 & 53.55 & 52.61 & 51.70 \\
\hline Line.115 & 2 & 55.20 & 53.29 & 53.56 & 52.61 & 51.70 \\
\hline Line.116 & 1 & 51.63 & 48.95 & 49.41 & 48.14 & 46.91 \\
\hline
\end{tabular}




\begin{tabular}{|c|c|c|c|c|c|c|}
\hline Line.116 & 2 & 51.63 & 48.96 & 49.42 & 48.14 & 46.91 \\
\hline Line.117 & 1 & 436.31 & 419.25 & 421.39 & 412.81 & 404.53 \\
\hline Line.117 & 2 & 436.30 & 419.24 & 421.38 & 412.80 & 404.52 \\
\hline Line.118 & 1 & 435.72 & 418.87 & 420.86 & 412.33 & 404.09 \\
\hline Line.118 & 2 & 435.71 & 418.87 & 420.86 & 412.32 & 404.09 \\
\hline Line.119 & 1 & 66.53 & 64.23 & 64.53 & 63.38 & 62.27 \\
\hline Line.119 & 2 & 66.54 & 64.24 & 64.54 & 63.39 & 62.28 \\
\hline Line.120 & 1 & 499.89 & 480.71 & 483.05 & 473.36 & 464.02 \\
\hline Line.120 & 2 & 499.89 & 480.71 & 483.04 & 473.36 & 464.02 \\
\hline Line.121 & 1 & 4.87 & 4.98 & 4.94 & 4.98 & 5.01 \\
\hline Line.121 & 2 & 4.87 & 4.98 & 4.94 & 4.97 & 5.01 \\
\hline Line.122 & 1 & 499.53 & 480.46 & 482.72 & 473.06 & 463.74 \\
\hline Line.122 & 2 & 499.53 & 480.46 & 482.72 & 473.06 & 463.74 \\
\hline Line.123 & 1 & 53.04 & 50.29 & 50.83 & 49.55 & 48.32 \\
\hline Line.123 & 2 & 53.04 & 50.29 & 50.83 & 49.56 & 48.32 \\
\hline Line.124 & 1 & 54.06 & 51.58 & 52.03 & 50.86 & 49.72 \\
\hline Line.124 & 2 & 54.06 & 51.58 & 52.03 & 50.86 & 49.72 \\
\hline Line.125 & 1 & 6.66 & 6.37 & 6.41 & 6.27 & 6.14 \\
\hline Line.125 & 2 & 6.66 & 6.37 & 6.41 & 6.27 & 6.14 \\
\hline Line.126 & 1 & 489.35 & 470.34 & 472.66 & 463.07 & 453.81 \\
\hline Line.126 & 2 & 489.34 & 470.33 & 472.66 & 463.06 & 453.81 \\
\hline Line.127 & 1 & 43.23 & 40.37 & 534.99 & 524.19 & 513.76 \\
\hline Line.127 & 2 & 43.24 & 40.38 & 534.98 & 524.18 & 513.76 \\
\hline Line.128 & 1 & 59.79 & 56.47 & 60.11 & 507.39 & 497.08 \\
\hline Line.128 & 2 & 59.80 & 56.48 & 60.12 & 507.38 & 497.08 \\
\hline Line.129 & 1 & 61.66 & 59.18 & 61.87 & 62.09 & 495.02 \\
\hline Line.129 & 2 & 61.67 & 59.19 & 61.88 & 62.09 & 495.01 \\
\hline Line.130 & 1 & 64.48 & 61.79 & 64.77 & 65.02 & 65.28 \\
\hline Line.130 & 2 & 64.49 & 61.79 & 64.77 & 65.03 & 65.29 \\
\hline Line.131 & 1 & 9.69 & 9.93 & 9.69 & 9.69 & 9.68 \\
\hline Line.131 & 2 & 9.69 & 9.93 & 9.69 & 9.68 & 9.68 \\
\hline Line.132 & 1 & 65.83 & 509.12 & 63.58 & 62.30 & 61.06 \\
\hline Line.132 & 2 & 65.84 & 509.11 & 63.59 & 62.30 & 61.06 \\
\hline Line.133 & 1 & 68.78 & 69.20 & 66.50 & 65.19 & 63.92 \\
\hline Line.133 & 2 & 68.80 & 69.22 & 66.51 & 65.20 & 63.94 \\
\hline Line.134 & 1 & 9.70 & 9.68 & 9.84 & 9.93 & 10.00 \\
\hline Line.134 & 2 & 9.70 & 9.68 & 9.84 & 9.92 & 10.00 \\
\hline
\end{tabular}




\subsubsection{Short Circuit Analysis 25\% DG Penetration}

Table 4-42: $I_{1}$ value, zone 1,3 phase fault, $25 \%$ penetration

\begin{tabular}{|c|c|c|c|c|c|c|c|c|}
\hline Element & Terminal & & & & ulty $\mathrm{Nc}$ & & & \\
\hline & & 705 & 713 & 704 & 714 & 720 & 706 & 707 \\
\hline Vsource & 1 & 14.91 & 15.02 & 14.77 & 14.71 & 14.39 & 14.12 & 13.80 \\
\hline Vsource & 2 & 14.91 & 15.02 & 14.77 & 14.71 & 14.39 & 14.12 & 13.80 \\
\hline Subxf & 1 & 14.91 & 15.02 & 14.77 & 14.71 & 14.39 & 14.12 & 13.80 \\
\hline Subxf & 2 & 714.60 & 719.55 & 707.61 & 705.02 & 689.54 & 676.68 & 661.23 \\
\hline Xfm1 & 1 & 0.00 & 0.00 & 0.00 & 0.00 & 0.00 & 0.00 & 0.00 \\
\hline Xfm1 & 2 & 0.00 & 0.00 & 0.00 & 0.00 & 0.00 & 0.00 & 0.00 \\
\hline Line.11 & 1 & 648.06 & 652.70 & 641.69 & 639.29 & 625.03 & 613.17 & 598.81 \\
\hline Line.11 & 2 & 648.06 & 652.70 & 641.69 & 639.29 & 625.03 & 613.17 & 598.81 \\
\hline Line.12 & 1 & 751.16 & 25.84 & 24.95 & 24.73 & 23.62 & 22.67 & 21.23 \\
\hline Line. 12 & 2 & 751.16 & 25.84 & 24.95 & 24.73 & 23.62 & 22.67 & 21.23 \\
\hline Line.13 & 1 & 31.14 & 754.06 & 738.27 & 734.65 & 714.47 & 697.53 & 675.37 \\
\hline Line.13 & 2 & 31.14 & 754.06 & 738.27 & 734.65 & 714.47 & 697.53 & 675.37 \\
\hline Line.14 & 1 & 76.14 & 78.48 & 75.31 & 74.43 & 70.62 & 67.31 & 61.22 \\
\hline Line.14 & 2 & 76.14 & 78.47 & 75.31 & 74.42 & 70.62 & 67.30 & 61.21 \\
\hline Line. 15 & 1 & 33.00 & 34.75 & 736.44 & 732.76 & 712.31 & 695.14 & 672.55 \\
\hline Line. 15 & 2 & 33.00 & 34.75 & 736.44 & 732.76 & 712.31 & 695.14 & 672.55 \\
\hline Line.16 & 1 & 10.51 & 11.10 & 11.28 & 11.20 & 735.11 & 717.21 & 693.65 \\
\hline Line.16 & 2 & 10.51 & 11.09 & 11.28 & 11.20 & 735.11 & 717.21 & 693.65 \\
\hline Line.17 & 1 & 0.97 & 0.80 & 0.78 & 0.81 & 0.76 & 726.90 & 1.10 \\
\hline Line.17 & 2 & 0.97 & 0.80 & 0.78 & 0.81 & 0.76 & 726.90 & 1.10 \\
\hline Line.18 & 1 & 0.97 & 0.80 & 0.78 & 0.81 & 0.76 & 0.74 & 1.10 \\
\hline Line. 18 & 2 & 0.97 & 0.80 & 0.78 & 0.81 & 0.76 & 0.74 & 1.10 \\
\hline Line.19 & 1 & 12.97 & 13.29 & 13.39 & 13.36 & 13.56 & 13.73 & 690.32 \\
\hline Line.19 & 2 & 12.97 & 13.29 & 13.39 & 13.35 & 13.56 & 13.73 & 690.32 \\
\hline Line.110 & 1 & 19.36 & 19.37 & 19.37 & 19.37 & 19.37 & 19.37 & 19.37 \\
\hline Line.110 & 2 & 19.36 & 19.37 & 19.37 & 19.37 & 19.37 & 19.37 & 19.37 \\
\hline Line.111 & 1 & 31.32 & 32.03 & 32.05 & 31.90 & 32.04 & 31.33 & 31.97 \\
\hline Line.111 & 2 & 31.32 & 32.03 & 32.05 & 31.90 & 32.04 & 31.33 & 31.97 \\
\hline Line.112 & 1 & 23.29 & 24.11 & 24.16 & 742.31 & 23.16 & 22.44 & 21.45 \\
\hline Line.112 & 2 & 23.29 & 24.11 & 24.16 & 742.31 & 23.16 & 22.44 & 21.45 \\
\hline Line.113 & 1 & 27.79 & 28.65 & 28.68 & 28.68 & 27.58 & 26.79 & 25.79 \\
\hline Line.113 & 2 & 27.79 & 28.65 & 28.68 & 28.68 & 27.58 & 26.79 & 25.79 \\
\hline Line.114 & 1 & 1.59 & 1.81 & 2.03 & 2.09 & 2.38 & 2.62 & 3.06 \\
\hline Line.114 & 2 & 1.59 & 1.81 & 2.03 & 2.09 & 2.38 & 2.62 & 3.06 \\
\hline
\end{tabular}




\begin{tabular}{|c|c|c|c|c|c|c|c|c|}
\hline \multirow[t]{2}{*}{ Element } & \multirow[t]{2}{*}{ Terminal } & \multicolumn{7}{|c|}{ Faulty Node } \\
\hline & & 705 & 713 & 704 & 714 & 720 & 706 & 707 \\
\hline Line.115 & 1 & 28.12 & 27.62 & 26.93 & 26.77 & 25.88 & 25.14 & 24.13 \\
\hline Line. 115 & 2 & 28.12 & 27.62 & 26.93 & 26.77 & 25.88 & 25.14 & 24.13 \\
\hline Line.116 & 1 & 20.62 & 21.23 & 20.30 & 20.06 & 18.91 & 17.93 & 16.31 \\
\hline Line.116 & 2 & 20.61 & 21.23 & 20.30 & 20.06 & 18.91 & 17.93 & 16.31 \\
\hline Line.117 & 1 & 55.62 & 57.33 & 55.12 & 54.49 & 51.87 & 49.58 & 45.13 \\
\hline Line.117 & 2 & 55.61 & 57.33 & 55.12 & 54.49 & 51.87 & 49.58 & 45.12 \\
\hline Line.118 & 1 & 57.37 & 58.93 & 56.85 & 56.26 & 53.75 & 51.54 & 47.37 \\
\hline Line.118 & 2 & 57.37 & 58.93 & 56.85 & 56.26 & 53.75 & 51.54 & 47.37 \\
\hline Line.119 & 1 & 32.74 & 33.11 & 32.39 & 32.22 & 31.30 & 30.52 & 29.45 \\
\hline Line.119 & 2 & 32.74 & 33.11 & 32.39 & 32.22 & 31.30 & 30.52 & 29.45 \\
\hline Line. 120 & 1 & 25.04 & 26.19 & 25.17 & 24.82 & 23.83 & 22.96 & 20.37 \\
\hline Line.120 & 2 & 25.04 & 26.19 & 25.17 & 24.82 & 23.82 & 22.96 & 20.37 \\
\hline Line.121 & 1 & 1.02 & 0.94 & 1.05 & 1.08 & 1.22 & 1.34 & 1.55 \\
\hline Line.121 & 2 & 1.02 & 0.94 & 1.05 & 1.08 & 1.22 & 1.34 & 1.55 \\
\hline Line.122 & 1 & 25.96 & 27.04 & 26.05 & 25.71 & 24.69 & 23.79 & 21.23 \\
\hline Line.122 & 2 & 25.96 & 27.04 & 26.05 & 25.71 & 24.69 & 23.78 & 21.23 \\
\hline Line.123 & 1 & 21.59 & 22.12 & 21.28 & 21.06 & 20.02 & 19.11 & 17.69 \\
\hline Line.123 & 2 & 21.59 & 22.12 & 21.28 & 21.06 & 20.02 & 19.11 & 17.69 \\
\hline Line.124 & 1 & 25.46 & 25.64 & 25.16 & 25.06 & 24.43 & 23.90 & 23.28 \\
\hline Line.124 & 2 & 25.46 & 25.64 & 25.16 & 25.06 & 24.43 & 23.90 & 23.28 \\
\hline Line. 125 & 1 & 2.97 & 2.70 & 3.02 & 3.12 & 3.52 & 3.88 & 4.52 \\
\hline Line. 125 & 2 & 2.97 & 2.70 & 3.02 & 3.12 & 3.52 & 3.88 & 4.52 \\
\hline Line.126 & 1 & 35.48 & 36.61 & 35.13 & 34.71 & 32.96 & 31.43 & 28.48 \\
\hline Line.126 & 2 & 35.48 & 36.61 & 35.13 & 34.71 & 32.95 & 31.42 & 28.48 \\
\hline Line.127 & 1 & 9.79 & 10.26 & 10.38 & 10.31 & 10.65 & 10.88 & 10.19 \\
\hline Line.127 & 2 & 9.79 & 10.26 & 10.37 & 10.30 & 10.65 & 10.87 & 10.19 \\
\hline Line.128 & 1 & 24.83 & 25.46 & 24.54 & 24.29 & 23.16 & 22.18 & 20.53 \\
\hline Line.128 & 2 & 24.83 & 25.46 & 24.54 & 24.29 & 23.16 & 22.18 & 20.53 \\
\hline Line.129 & 1 & 27.70 & 28.07 & 27.40 & 27.23 & 26.38 & 25.65 & 24.53 \\
\hline Line.129 & 2 & 27.69 & 28.07 & 27.40 & 27.23 & 26.38 & 25.65 & 24.53 \\
\hline Line.130 & 1 & 29.76 & 29.97 & 29.47 & 29.36 & 28.72 & 28.17 & 27.46 \\
\hline Line.130 & 2 & 29.76 & 29.97 & 29.47 & 29.36 & 28.72 & 28.17 & 27.46 \\
\hline Line.131 & 1 & 2.12 & 1.95 & 2.17 & 2.23 & 2.51 & 2.75 & 3.18 \\
\hline Line.131 & 2 & 2.12 & 1.95 & 2.17 & 2.23 & 2.51 & 2.75 & 3.18 \\
\hline Line.132 & 1 & 28.04 & 28.47 & 27.66 & 27.46 & 26.43 & 25.56 & 24.34 \\
\hline Line.132 & 2 & 28.04 & 28.47 & 27.66 & 27.46 & 26.43 & 25.56 & 24.34 \\
\hline Line.133 & 1 & 30.13 & 30.39 & 29.78 & 29.65 & 28.85 & 28.19 & 27.39 \\
\hline Line.133 & 2 & 30.13 & 30.39 & 29.78 & 29.64 & 28.85 & 28.19 & 27.39 \\
\hline Line.134 & 1 & 2.12 & 1.94 & 2.16 & 2.23 & 2.50 & 2.75 & 3.17 \\
\hline Line.134 & 2 & 2.12 & 1.94 & 2.16 & 2.23 & 2.50 & 2.75 & 3.17 \\
\hline
\end{tabular}


Table 4-43: $I_{r}$ value, zone 1, SLG fault, $25 \%$ penetration

\begin{tabular}{|c|c|c|c|c|c|c|c|c|}
\hline Element & Terminal & & & & lity Nod & & & \\
\hline & & 705 & 713 & 704 & 714 & 720 & 706 & 707 \\
\hline Vsource & 1 & 0.00 & 0.00 & 0.00 & 0.00 & 0.00 & 0.00 & 0.00 \\
\hline Vsource & 2 & 0.00 & 0.00 & 0.00 & 0.00 & 0.00 & 0.00 & 0.00 \\
\hline Subxf & 1 & 0.00 & 0.00 & 0.00 & 0.00 & 0.00 & 0.00 & 0.00 \\
\hline Subxf & 2 & 0.00 & 0.00 & 0.00 & 0.00 & 0.00 & 0.00 & 0.00 \\
\hline Xfm1 & 1 & 0.00 & 0.00 & 0.00 & 0.00 & 0.00 & 0.00 & 0.00 \\
\hline Xfm1 & 2 & 0.00 & 0.00 & 0.00 & 0.00 & 0.00 & 0.00 & 0.00 \\
\hline Line.11 & 1 & 0.26 & 0.26 & 0.25 & 0.25 & 0.25 & 0.24 & 0.23 \\
\hline Line.11 & 2 & 0.26 & 0.26 & 0.25 & 0.25 & 0.25 & 0.24 & 0.23 \\
\hline Line.12 & 1 & 475.26 & 77.69 & 76.31 & 76.01 & 73.98 & 72.15 & 70.54 \\
\hline Line. 12 & 2 & 475.26 & 77.69 & 76.31 & 76.01 & 73.98 & 72.15 & 70.54 \\
\hline Line.13 & 1 & 152.57 & 402.96 & 395.80 & 394.20 & 383.71 & 374.21 & 365.82 \\
\hline Line.13 & 2 & 152.57 & 402.96 & 395.80 & 394.20 & 383.71 & 374.21 & 365.82 \\
\hline Line.14 & 1 & 323.08 & 325.68 & 319.90 & 318.60 & 310.12 & 302.44 & 295.66 \\
\hline Line.14 & 2 & 323.08 & 325.68 & 319.90 & 318.60 & 310.12 & 302.44 & 295.66 \\
\hline Line.15 & 1 & 152.59 & 155.25 & 395.78 & 394.18 & 383.68 & 374.19 & 365.80 \\
\hline Line.15 & 2 & 152.59 & 155.25 & 395.78 & 394.18 & 383.68 & 374.19 & 365.80 \\
\hline Line.16 & 1 & 74.79 & 76.09 & 75.76 & 75.45 & 459.96 & 448.57 & 438.52 \\
\hline Line.16 & 2 & 74.79 & 76.09 & 75.76 & 75.45 & 459.96 & 448.57 & 438.52 \\
\hline Line.17 & 1 & 0.05 & 0.05 & 0.05 & 0.05 & 0.05 & 521.60 & 0.05 \\
\hline Line.17 & 2 & 0.05 & 0.05 & 0.05 & 0.05 & 0.05 & 521.60 & 0.05 \\
\hline Line.18 & 1 & 0.01 & 0.01 & 0.01 & 0.01 & 0.01 & 0.01 & 0.01 \\
\hline Line.18 & 2 & 0.01 & 0.01 & 0.01 & 0.01 & 0.01 & 0.01 & 0.01 \\
\hline Line.19 & 1 & 74.89 & 76.20 & 75.86 & 75.55 & 75.13 & 73.27 & 438.42 \\
\hline Line.19 & 2 & 74.89 & 76.20 & 75.86 & 75.55 & 75.13 & 73.27 & 438.42 \\
\hline Line. 110 & 1 & 0.01 & 0.01 & 0.01 & 0.01 & 0.01 & 0.01 & 0.01 \\
\hline Line.110 & 2 & 0.01 & 0.01 & 0.01 & 0.01 & 0.01 & 0.01 & 0.01 \\
\hline Line.111 & 1 & 74.95 & 76.25 & 75.92 & 75.61 & 75.18 & 73.32 & 74.01 \\
\hline Line.111 & 2 & 74.95 & 76.25 & 75.92 & 75.61 & 75.18 & 73.32 & 74.01 \\
\hline Line.112 & 1 & 77.84 & 79.20 & 78.85 & 469.52 & 76.44 & 74.55 & 72.88 \\
\hline Line.112 & 2 & 77.84 & 79.20 & 78.85 & 469.52 & 76.44 & 74.55 & 72.88 \\
\hline Line.113 & 1 & 77.84 & 79.20 & 78.85 & 78.74 & 76.44 & 74.55 & 72.88 \\
\hline Line.113 & 2 & 77.84 & 79.20 & 78.85 & 78.74 & 76.44 & 74.55 & 72.88 \\
\hline Line.114 & 1 & 0.01 & 0.01 & 0.01 & 0.01 & 0.01 & 0.01 & 0.01 \\
\hline Line.114 & 2 & 0.01 & 0.01 & 0.01 & 0.01 & 0.01 & 0.01 & 0.01 \\
\hline Line.115 & 1 & 78.10 & 77.73 & 76.35 & 76.04 & 74.01 & 72.18 & 70.57 \\
\hline Line.115 & 2 & 78.10 & 77.73 & 76.35 & 76.04 & 74.01 & 72.18 & 70.57 \\
\hline Line.116 & 1 & 80.56 & 81.21 & 79.77 & 79.44 & 77.33 & 75.41 & 73.73 \\
\hline
\end{tabular}




\begin{tabular}{|c|c|c|c|c|c|c|c|c|}
\hline \multirow[t]{2}{*}{ Element } & \multirow[t]{2}{*}{ Terminal } & \multicolumn{7}{|c|}{ Faulty Node } \\
\hline & & 705 & 713 & 704 & 714 & 720 & 706 & 707 \\
\hline Line.116 & 2 & 80.56 & 81.21 & 79.77 & 79.44 & 77.33 & 75.41 & 73.73 \\
\hline Line.117 & 1 & 242.70 & 244.66 & 240.31 & 239.34 & 232.97 & 227.20 & 222.11 \\
\hline Line.117 & 2 & 242.70 & 244.66 & 240.31 & 239.34 & 232.97 & 227.20 & 222.11 \\
\hline Line.118 & 1 & 242.74 & 244.70 & 240.35 & 239.38 & 233.00 & 227.24 & 222.14 \\
\hline Line.118 & 2 & 242.74 & 244.70 & 240.35 & 239.38 & 233.00 & 227.24 & 222.14 \\
\hline Line.119 & 1 & 80.81 & 81.46 & 80.02 & 79.69 & 77.57 & 75.65 & 73.96 \\
\hline Line.119 & 2 & 80.81 & 81.46 & 80.02 & 79.69 & 77.57 & 75.65 & 73.96 \\
\hline Line. 120 & 1 & 161.96 & 163.27 & 160.37 & 159.72 & 155.47 & 151.62 & 148.22 \\
\hline Line. 120 & 2 & 161.96 & 163.27 & 160.37 & 159.72 & 155.47 & 151.62 & 148.22 \\
\hline Line.121 & 1 & 0.02 & 0.02 & 0.02 & 0.02 & 0.02 & 0.02 & 0.02 \\
\hline Line.121 & 2 & 0.02 & 0.02 & 0.02 & 0.02 & 0.02 & 0.02 & 0.02 \\
\hline Line. 122 & 1 & 162.00 & 163.31 & 160.41 & 159.76 & 155.50 & 151.65 & 148.25 \\
\hline Line.122 & 2 & 162.00 & 163.31 & 160.41 & 159.76 & 155.50 & 151.65 & 148.25 \\
\hline Line. 123 & 1 & 80.57 & 81.22 & 79.78 & 79.46 & 77.34 & 75.43 & 73.74 \\
\hline Line.123 & 2 & 80.57 & 81.22 & 79.78 & 79.46 & 77.34 & 75.43 & 73.74 \\
\hline Line.124 & 1 & 80.60 & 81.25 & 79.81 & 79.48 & 77.37 & 75.45 & 73.76 \\
\hline Line.124 & 2 & 80.60 & 81.25 & 79.81 & 79.48 & 77.37 & 75.45 & 73.76 \\
\hline Line. 125 & 1 & 0.01 & 0.01 & 0.01 & 0.01 & 0.01 & 0.01 & 0.01 \\
\hline Line.125 & 2 & 0.01 & 0.01 & 0.01 & 0.01 & 0.01 & 0.01 & 0.01 \\
\hline Line.126 & 1 & 162.02 & 163.33 & 160.43 & 159.78 & 155.52 & 151.67 & 148.27 \\
\hline Line.126 & 2 & 162.02 & 163.33 & 160.43 & 159.78 & 155.52 & 151.67 & 148.27 \\
\hline Line.127 & 1 & 80.78 & 81.43 & 79.99 & 79.66 & 77.54 & 75.62 & 73.93 \\
\hline Line.127 & 2 & 80.78 & 81.43 & 79.99 & 79.66 & 77.54 & 75.62 & 73.93 \\
\hline Line. 128 & 1 & 80.82 & 81.48 & 80.03 & 79.70 & 77.58 & 75.66 & 73.96 \\
\hline Line.128 & 2 & 80.82 & 81.48 & 80.03 & 79.70 & 77.58 & 75.66 & 73.96 \\
\hline Line.129 & 1 & 80.85 & 81.50 & 80.05 & 79.73 & 77.61 & 75.68 & 73.99 \\
\hline Line.129 & 2 & 80.85 & 81.50 & 80.05 & 79.73 & 77.61 & 75.68 & 73.99 \\
\hline Line. 130 & 1 & 80.88 & 81.54 & 80.09 & 79.76 & 77.64 & 75.72 & 74.02 \\
\hline Line.130 & 2 & 80.88 & 81.54 & 80.09 & 79.76 & 77.64 & 75.72 & 74.02 \\
\hline Line.131 & 1 & 0.01 & 0.01 & 0.01 & 0.01 & 0.01 & 0.01 & 0.01 \\
\hline Line.131 & 2 & 0.01 & 0.01 & 0.01 & 0.01 & 0.01 & 0.01 & 0.01 \\
\hline Line.132 & 1 & 81.27 & 81.93 & 80.47 & 80.15 & 78.01 & 76.08 & 74.38 \\
\hline Line.132 & 2 & 81.27 & 81.93 & 80.47 & 80.15 & 78.01 & 76.08 & 74.38 \\
\hline Line.133 & 1 & 81.31 & 81.97 & 80.51 & 80.18 & 78.05 & 76.12 & 74.41 \\
\hline Line.133 & 2 & 81.31 & 81.97 & 80.51 & 80.18 & 78.05 & 76.12 & 74.41 \\
\hline Line.134 & 1 & 0.01 & 0.01 & 0.01 & 0.01 & 0.01 & 0.01 & 0.01 \\
\hline Line.134 & 2 & 0.01 & 0.01 & 0.01 & 0.01 & 0.01 & 0.01 & 0.01 \\
\hline
\end{tabular}


Table 4-44: $I_{2}$ value, zone 1, LL fault, $25 \%$ penetration

\begin{tabular}{|c|c|c|c|c|c|c|c|c|}
\hline \multirow[t]{2}{*}{ Element } & \multirow[t]{2}{*}{ Terminal } & \multicolumn{7}{|c|}{ Faulty Node } \\
\hline & & 705 & 713 & 704 & 714 & 720 & 706 & 707 \\
\hline Vsource & 1 & 6.29 & 6.36 & 6.23 & 6.20 & 6.03 & 5.87 & 5.68 \\
\hline Vsource & 2 & 6.29 & 6.36 & 6.23 & 6.20 & 6.03 & 5.87 & 5.68 \\
\hline Subxf & 1 & 6.29 & 6.36 & 6.23 & 6.20 & 6.03 & 5.87 & 5.68 \\
\hline Subxf & 2 & 301.27 & 304.98 & 298.57 & 296.96 & 288.75 & 281.33 & 272.08 \\
\hline Xfm1 & 1 & 0.00 & 0.00 & 0.00 & 0.00 & 0.00 & 0.00 & 0.00 \\
\hline Xfm1 & 2 & 0.00 & 0.00 & 0.00 & 0.00 & 0.00 & 0.00 & 0.00 \\
\hline Line.11 & 1 & 274.07 & 277.43 & 271.59 & 270.13 & 262.65 & 255.89 & 247.50 \\
\hline Line.11 & 2 & 274.04 & 277.40 & 271.57 & 270.10 & 262.62 & 255.86 & 247.47 \\
\hline Line. 12 & 1 & 384.48 & 25.51 & 25.19 & 25.12 & 24.70 & 24.33 & 23.97 \\
\hline Line.12 & 2 & 384.48 & 25.52 & 25.20 & 25.13 & 24.71 & 24.33 & 23.97 \\
\hline Line.13 & 1 & 59.69 & 356.81 & 349.17 & 347.23 & 337.38 & 328.38 & 317.03 \\
\hline Line.13 & 2 & 59.69 & 356.80 & 349.16 & 347.23 & 337.38 & 328.37 & 317.02 \\
\hline Line.14 & 1 & 58.62 & 59.38 & 57.26 & 56.84 & 54.40 & 52.35 & 50.18 \\
\hline Line.14 & 2 & 58.65 & 59.41 & 57.29 & 56.87 & 54.43 & 52.37 & 50.21 \\
\hline Line. 15 & 1 & 57.96 & 58.84 & 348.68 & 346.76 & 336.89 & 327.89 & 316.76 \\
\hline Line. 15 & 2 & 57.97 & 58.85 & 348.67 & 346.75 & 336.88 & 327.88 & 316.75 \\
\hline Line.16 & 1 & 42.58 & 42.78 & 42.81 & 42.78 & 354.63 & 344.99 & 332.86 \\
\hline Line.16 & 2 & 42.59 & 42.79 & 42.82 & 42.78 & 354.61 & 344.98 & 332.85 \\
\hline Line.17 & 1 & 4.21 & 4.27 & 4.29 & 4.28 & 4.31 & 381.32 & 4.24 \\
\hline Line.17 & 2 & 4.22 & 4.28 & 4.30 & 4.29 & 4.32 & 381.31 & 4.24 \\
\hline Line.18 & 1 & 4.22 & 4.28 & 4.30 & 4.29 & 4.32 & 4.34 & 4.24 \\
\hline Line. 18 & 2 & 4.22 & 4.28 & 4.30 & 4.29 & 4.32 & 4.34 & 4.24 \\
\hline Line.19 & 1 & 36.49 & 36.66 & 36.68 & 36.65 & 36.72 & 36.50 & 336.90 \\
\hline Line.19 & 2 & 36.50 & 36.67 & 36.69 & 36.66 & 36.73 & 36.51 & 336.89 \\
\hline Line.110 & 1 & 18.04 & 18.12 & 18.12 & 18.11 & 18.13 & 18.06 & 18.13 \\
\hline Line.110 & 2 & 18.04 & 18.12 & 18.12 & 18.11 & 18.13 & 18.06 & 18.13 \\
\hline Line.111 & 1 & 22.23 & 22.73 & 22.80 & 22.71 & 22.89 & 22.44 & 23.02 \\
\hline Line.111 & 2 & 22.24 & 22.74 & 22.81 & 22.72 & 22.90 & 22.45 & 23.03 \\
\hline Line.112 & 1 & 19.33 & 20.06 & 20.17 & 387.10 & 19.53 & 19.01 & 18.15 \\
\hline Line.112 & 2 & 19.33 & 20.06 & 20.17 & 387.10 & 19.53 & 19.01 & 18.15 \\
\hline Line.113 & 1 & 19.50 & 20.39 & 20.51 & 20.53 & 19.63 & 18.96 & 18.00 \\
\hline Line.113 & 2 & 19.51 & 20.40 & 20.52 & 20.53 & 19.63 & 18.97 & 18.01 \\
\hline Line.114 & 1 & 9.46 & 9.52 & 9.59 & 9.62 & 9.70 & 9.78 & 9.94 \\
\hline Line.114 & 2 & 9.46 & 9.52 & 9.59 & 9.61 & 9.70 & 9.78 & 9.94 \\
\hline Line.115 & 1 & 26.20 & 25.92 & 25.59 & 25.50 & 25.08 & 24.69 & 24.22 \\
\hline Line.115 & 2 & 26.20 & 25.92 & 25.59 & 25.51 & 25.08 & 24.70 & 24.22 \\
\hline Line.116 & 1 & 16.62 & 16.95 & 16.41 & 16.27 & 15.60 & 14.98 & 14.15 \\
\hline
\end{tabular}




\begin{tabular}{|c|c|c|c|c|c|c|c|c|}
\hline \multirow[t]{2}{*}{ Element } & \multirow[t]{2}{*}{ Terminal } & \multicolumn{7}{|c|}{ Faulty Node } \\
\hline & & 705 & 713 & 704 & 714 & 720 & 706 & 707 \\
\hline Line.116 & 2 & 16.63 & 16.95 & 16.41 & 16.27 & 15.60 & 14.98 & 14.15 \\
\hline Line.117 & 1 & 42.59 & 43.02 & 41.40 & 41.12 & 39.32 & 37.88 & 36.61 \\
\hline Line.117 & 2 & 42.60 & 43.03 & 41.40 & 41.13 & 39.33 & 37.89 & 36.62 \\
\hline Line.118 & 1 & 40.14 & 40.75 & 39.36 & 39.06 & 37.46 & 36.05 & 34.29 \\
\hline Line.118 & 2 & 40.14 & 40.75 & 39.36 & 39.06 & 37.47 & 36.05 & 34.29 \\
\hline Line.119 & 1 & 26.85 & 27.03 & 26.70 & 26.62 & 26.19 & 25.79 & 25.32 \\
\hline Line.119 & 2 & 26.86 & 27.04 & 26.71 & 26.62 & 26.19 & 25.80 & 25.33 \\
\hline Line. 120 & 1 & 19.07 & 19.18 & 17.45 & 17.25 & 15.55 & 14.44 & 14.21 \\
\hline Line. 120 & 2 & 19.07 & 19.18 & 17.45 & 17.25 & 15.56 & 14.44 & 14.21 \\
\hline Line.121 & 1 & 4.72 & 4.68 & 4.72 & 4.73 & 4.77 & 4.81 & 4.89 \\
\hline Line.121 & 2 & 4.72 & 4.68 & 4.71 & 4.73 & 4.77 & 4.81 & 4.89 \\
\hline Line.122 & 1 & 16.04 & 16.31 & 14.76 & 14.53 & 12.98 & 11.84 & 11.01 \\
\hline Line.122 & 2 & 16.04 & 16.32 & 14.76 & 14.53 & 12.99 & 11.84 & 11.01 \\
\hline Line. 123 & 1 & 16.93 & 17.30 & 16.87 & 16.74 & 16.19 & 15.64 & 14.72 \\
\hline Line.123 & 2 & 16.94 & 17.31 & 16.88 & 16.74 & 16.19 & 15.64 & 14.72 \\
\hline Line.124 & 1 & 17.12 & 17.37 & 17.20 & 17.12 & 16.82 & 16.46 & 15.76 \\
\hline Line. 124 & 2 & 17.12 & 17.38 & 17.21 & 17.12 & 16.83 & 16.47 & 15.77 \\
\hline Line. 125 & 1 & 7.23 & 7.32 & 7.17 & 7.13 & 6.94 & 6.76 & 6.53 \\
\hline Line. 125 & 2 & 7.23 & 7.32 & 7.17 & 7.13 & 6.94 & 6.76 & 6.53 \\
\hline Line.126 & 1 & 25.49 & 25.84 & 24.67 & 24.45 & 23.11 & 22.02 & 21.03 \\
\hline Line.126 & 2 & 25.49 & 25.85 & 24.67 & 24.46 & 23.12 & 22.03 & 21.04 \\
\hline Line. 127 & 1 & 3.46 & 3.44 & 2.17 & 2.07 & 1.06 & 1.10 & 2.27 \\
\hline Line.127 & 2 & 3.46 & 3.43 & 2.17 & 2.07 & 1.05 & 1.09 & 2.26 \\
\hline Line. 128 & 1 & 18.09 & 18.43 & 17.78 & 17.63 & 16.79 & 16.05 & 15.22 \\
\hline Line.128 & 2 & 18.09 & 18.43 & 17.79 & 17.63 & 16.80 & 16.06 & 15.22 \\
\hline Line.129 & 1 & 19.95 & 20.00 & 19.71 & 19.66 & 19.27 & 18.94 & 18.74 \\
\hline Line.129 & 2 & 19.96 & 20.01 & 19.72 & 19.67 & 19.27 & 18.95 & 18.75 \\
\hline Line.130 & 1 & 19.83 & 20.04 & 19.62 & 19.51 & 18.96 & 18.46 & 17.89 \\
\hline Line.130 & 2 & 19.83 & 20.05 & 19.62 & 19.52 & 18.96 & 18.46 & 17.90 \\
\hline Line.131 & 1 & 9.52 & 9.44 & 9.52 & 9.54 & 9.63 & 9.71 & 9.87 \\
\hline Line. 131 & 2 & 9.52 & 9.44 & 9.51 & 9.54 & 9.63 & 9.71 & 9.87 \\
\hline Line.132 & 1 & 22.95 & 23.08 & 22.74 & 22.66 & 22.20 & 21.80 & 21.42 \\
\hline Line. 132 & 2 & 22.96 & 23.09 & 22.74 & 22.67 & 22.21 & 21.81 & 21.42 \\
\hline Line.133 & 1 & 24.20 & 24.42 & 24.03 & 23.93 & 23.43 & 22.97 & 22.41 \\
\hline Line.133 & 2 & 24.21 & 24.44 & 24.05 & 23.95 & 23.45 & 22.99 & 22.43 \\
\hline Line.134 & 1 & 9.53 & 9.45 & 9.52 & 9.55 & 9.64 & 9.72 & 9.88 \\
\hline Line.134 & 2 & 9.53 & 9.45 & 9.52 & 9.55 & 9.63 & 9.71 & 9.87 \\
\hline
\end{tabular}


Table 4-45: $I_{1}$ value, zone 2,3 phase fault, $25 \%$ penetration

\begin{tabular}{|c|c|c|c|c|c|c|}
\hline Element & Terminal & \multicolumn{5}{|c|}{ Faulty Node } \\
\hline & & $\mathbf{7 4 4}$ & $\mathbf{7 2 7}$ & $\mathbf{7 3 0}$ & $\mathbf{7 0 9}$ & $\mathbf{7 0 8}$ \\
\hline Vsource & 1 & 14.55 & 14.68 & 14.56 & 14.48 & 14.33 \\
\hline Vsource & 2 & 14.55 & 14.68 & 14.56 & 14.48 & 14.33 \\
\hline Subxf & 1 & 14.55 & 14.68 & 14.56 & 14.48 & 14.33 \\
\hline Subxf & 2 & 697.02 & 703.20 & 697.88 & 693.60 & 686.59 \\
\hline Xfm1 & 1 & 0.00 & 0.00 & 0.00 & 0.00 & 0.00 \\
\hline Xfm1 & 2 & 0.00 & 0.00 & 0.00 & 0.00 & 0.00 \\
\hline Line.11 & 1 & 631.96 & 637.67 & 632.80 & 628.86 & 622.40 \\
\hline Line.11 & 2 & 631.96 & 637.67 & 632.80 & 628.86 & 622.40 \\
\hline Line.12 & 1 & 24.27 & 24.73 & 24.44 & 24.13 & 23.61 \\
\hline Line.12 & 2 & 24.27 & 24.73 & 24.44 & 24.13 & 23.61 \\
\hline Line.13 & 1 & 30.37 & 30.94 & 30.84 & 30.46 & 29.82 \\
\hline Line.13 & 2 & 30.37 & 30.94 & 30.84 & 30.45 & 29.82 \\
\hline Line.14 & 1 & 683.42 & 690.46 & 684.77 & 679.93 & 671.97 \\
\hline Line.14 & 2 & 683.42 & 690.46 & 684.78 & 679.93 & 671.97 \\
\hline Line.15 & 1 & 32.20 & 32.73 & 32.58 & 32.23 & 31.63 \\
\hline Line.15 & 2 & 32.20 & 32.72 & 32.58 & 32.22 & 31.63 \\
\hline Line.16 & 1 & 11.35 & 11.31 & 11.66 & 11.69 & 11.72 \\
\hline Line.16 & 2 & 11.35 & 11.31 & 11.66 & 11.68 & 11.72 \\
\hline Line.17 & 1 & 1.05 & 1.00 & 1.02 & 1.05 & 1.12 \\
\hline Line.17 & 2 & 1.05 & 1.00 & 1.02 & 1.05 & 1.12 \\
\hline Line.18 & 1 & 1.05 & 1.00 & 1.02 & 1.05 & 1.12 \\
\hline Line.18 & 2 & 1.05 & 1.00 & 1.02 & 1.05 & 1.12 \\
\hline Line.19 & 1 & 13.41 & 13.41 & 13.60 & 13.59 & 13.56 \\
\hline Line.19 & 2 & 13.40 & 13.41 & 13.59 & 13.58 & 13.55 \\
\hline Line.110 & 1 & 19.36 & 19.36 & 19.36 & 19.36 & 19.36 \\
\hline Line.110 & 2 & 19.36 & 19.36 & 19.36 & 19.36 & 19.36 \\
\hline Line.111 & 1 & 30.47 & 30.79 & 30.53 & 30.32 & 29.96 \\
\hline Line.111 & 2 & 30.47 & 30.79 & 30.53 & 30.32 & 29.96 \\
\hline Line.112 & 1 & 22.45 & 22.78 & 22.54 & 22.31 & 21.92 \\
\hline Line.112 & 2 & 22.45 & 22.78 & 22.54 & 22.31 & 21.92 \\
\hline Line.113 & 1 & 26.83 & 27.19 & 26.90 & 26.65 & 26.24 \\
\hline Line.113 & 2 & 26.83 & 27.19 & 26.90 & 26.65 & 26.24 \\
\hline Line.114 & 1 & 2.18 & 2.06 & 2.10 & 2.18 & 2.31 \\
\hline Line.114 & 2 & 2.18 & 2.06 & 2.10 & 2.18 & 2.31 \\
\hline Line.115 & 1 & 26.35 & 26.71 & 26.44 & 26.19 & 25.78 \\
\hline Line.115 & 2 & 26.35 & 26.71 & 26.44 & 26.19 & 25.78 \\
\hline Line.116 & 1 & 737.38 & 745.96 & 21.10 & 20.77 & 20.22 \\
\hline
\end{tabular}




\begin{tabular}{|l|c|c|c|c|c|c|}
\hline Element & Terminal & \multicolumn{5}{|c|}{ Faulty Node } \\
\hline & & $\mathbf{7 4 4}$ & $\mathbf{7 2 7}$ & $\mathbf{7 3 0}$ & $\mathbf{7 0 9}$ & $\mathbf{7 0 8}$ \\
\hline Line.116 & 2 & 737.38 & 745.96 & 21.10 & 20.77 & 20.22 \\
\hline Line.117 & 1 & 56.59 & 57.77 & 705.43 & 700.22 & 691.66 \\
\hline Line.117 & 2 & 56.59 & 57.76 & 705.43 & 700.22 & 691.66 \\
\hline Line.118 & 1 & 58.22 & 59.34 & 61.66 & 698.75 & 690.07 \\
\hline Line.118 & 2 & 58.22 & 59.34 & 61.66 & 698.75 & 690.07 \\
\hline Line.119 & 1 & 32.83 & 33.22 & 33.81 & 33.83 & 33.38 \\
\hline Line.119 & 2 & 32.83 & 33.22 & 33.81 & 33.83 & 33.37 \\
\hline Line.120 & 1 & 25.89 & 26.44 & 28.07 & 28.24 & 722.76 \\
\hline Line.120 & 2 & 25.89 & 26.44 & 28.07 & 28.24 & 722.76 \\
\hline Line.121 & 1 & 0.97 & 0.91 & 0.78 & 0.77 & 0.75 \\
\hline Line.121 & 2 & 0.97 & 0.91 & 0.78 & 0.77 & 0.75 \\
\hline Line.122 & 1 & 26.75 & 27.28 & 28.81 & 28.96 & 29.18 \\
\hline Line.122 & 2 & 26.75 & 27.28 & 28.81 & 28.96 & 29.18 \\
\hline Line.123 & 1 & 736.55 & 22.98 & 21.98 & 21.67 & 21.17 \\
\hline Line.123 & 2 & 736.55 & 22.98 & 21.98 & 21.67 & 21.17 \\
\hline Line.124 & 1 & 26.04 & 26.04 & 25.52 & 25.34 & 25.05 \\
\hline Line.124 & 2 & 26.04 & 26.04 & 25.52 & 25.34 & 25.05 \\
\hline Line.125 & 1 & 2.30 & 2.33 & 2.73 & 2.85 & 3.04 \\
\hline Line.125 & 2 & 2.30 & 2.33 & 2.73 & 2.85 & 3.04 \\
\hline Line.126 & 1 & 36.11 & 36.90 & 38.57 & 38.70 & 38.86 \\
\hline Line.126 & 2 & 36.10 & 36.90 & 38.57 & 38.70 & 38.86 \\
\hline Line.127 & 1 & 10.36 & 10.30 & 10.87 & 10.98 & 11.16 \\
\hline Line.127 & 2 & 10.36 & 10.30 & 10.87 & 10.98 & 11.16 \\
\hline Line.128 & 1 & 25.11 & 25.60 & 26.55 & 26.60 & 26.67 \\
\hline Line.128 & 2 & 25.11 & 25.60 & 26.55 & 26.60 & 26.67 \\
\hline Line.129 & 1 & 27.86 & 28.21 & 28.82 & 28.85 & 28.88 \\
\hline Line.129 & 2 & 27.86 & 28.21 & 28.82 & 28.85 & 28.88 \\
\hline Line.130 & 1 & 29.80 & 30.07 & 30.42 & 30.43 & 30.43 \\
\hline Line.130 & 2 & 29.80 & 30.07 & 30.42 & 30.43 & 30.43 \\
\hline Line.131 & 1 & 2.01 & 1.90 & 1.63 & 1.61 & 1.58 \\
\hline Line.131 & 2 & 2.01 & 1.90 & 1.63 & 1.61 & 1.58 \\
\hline Line.132 & 1 & 28.20 & 28.63 & 29.33 & 29.37 & 29.40 \\
\hline Line.132 & 2 & 28.20 & 28.63 & 29.33 & 29.37 & 29.40 \\
\hline Line.133 & 1 & 30.17 & 30.51 & 30.95 & 30.96 & 30.96 \\
\hline Line.133 & 2 & 30.17 & 30.51 & 30.95 & 30.96 & 30.96 \\
\hline Line.134 & 1 & 2.01 & 1.89 & 1.63 & 1.60 & 1.57 \\
\hline Line.134 & 2 & 2.01 & 1.89 & 1.63 & 1.60 & 1.57 \\
\hline
\end{tabular}


Table 4-46: $I_{r}$ value, zone 2, SLG fault, $25 \%$ penetration

\begin{tabular}{|c|c|c|c|c|c|c|}
\hline Element & Terminal & \multicolumn{5}{|c|}{ Faulty Node } \\
\hline & & $\mathbf{7 4 4}$ & $\mathbf{7 2 7}$ & $\mathbf{7 3 0}$ & $\mathbf{7 0 9}$ & $\mathbf{7 0 8}$ \\
\hline Vsource & 1 & 0.00 & 0.00 & 0.00 & 0.00 & 0.00 \\
\hline Vsource & 2 & 0.00 & 0.00 & 0.00 & 0.00 & 0.00 \\
\hline Subxf & 1 & 0.00 & 0.00 & 0.00 & 0.00 & 0.00 \\
\hline Subxf & 2 & 0.00 & 0.00 & 0.00 & 0.00 & 0.00 \\
\hline Xfm1 & 1 & 0.00 & 0.00 & 0.00 & 0.00 & 0.00 \\
\hline Xfm1 & 2 & 0.00 & 0.00 & 0.00 & 0.00 & 0.00 \\
\hline Line.11 & 1 & 0.25 & 0.25 & 0.25 & 0.25 & 0.25 \\
\hline Line.11 & 2 & 0.25 & 0.25 & 0.25 & 0.25 & 0.25 \\
\hline Line.12 & 1 & 75.87 & 76.67 & 76.23 & 75.78 & 74.96 \\
\hline Line.12 & 2 & 75.87 & 76.67 & 76.23 & 75.78 & 74.96 \\
\hline Line.13 & 1 & 150.18 & 151.77 & 150.89 & 150.00 & 148.37 \\
\hline Line.13 & 2 & 150.18 & 151.77 & 150.89 & 150.00 & 148.37 \\
\hline Line.14 & 1 & 225.68 & 228.07 & 226.75 & 225.41 & 222.97 \\
\hline Line.14 & 2 & 225.68 & 228.07 & 226.75 & 225.41 & 222.97 \\
\hline Line.15 & 1 & 150.20 & 151.79 & 150.91 & 150.02 & 148.40 \\
\hline Line.15 & 2 & 150.20 & 151.79 & 150.91 & 150.02 & 148.40 \\
\hline Line.16 & 1 & 73.62 & 74.40 & 73.97 & 73.53 & 72.73 \\
\hline Line.16 & 2 & 73.62 & 74.40 & 73.97 & 73.53 & 72.73 \\
\hline Line.17 & 1 & 0.05 & 0.05 & 0.05 & 0.05 & 0.05 \\
\hline Line.17 & 2 & 0.05 & 0.05 & 0.05 & 0.05 & 0.05 \\
\hline Line.18 & 1 & 0.01 & 0.01 & 0.01 & 0.01 & 0.01 \\
\hline Line.18 & 2 & 0.01 & 0.01 & 0.01 & 0.01 & 0.01 \\
\hline Line.19 & 1 & 73.72 & 74.50 & 74.07 & 73.63 & 72.83 \\
\hline Line.19 & 2 & 73.72 & 74.50 & 74.07 & 73.63 & 72.83 \\
\hline Line.110 & 1 & 0.01 & 0.01 & 0.01 & 0.01 & 0.01 \\
\hline Line.110 & 2 & 0.01 & 0.01 & 0.01 & 0.01 & 0.01 \\
\hline Line.111 & 1 & 73.77 & 74.56 & 74.12 & 73.69 & 72.89 \\
\hline Line.111 & 2 & 73.77 & 74.56 & 74.12 & 73.69 & 72.89 \\
\hline Line.112 & 1 & 76.62 & 77.43 & 76.99 & 76.53 & 75.70 \\
\hline Line.112 & 2 & 76.62 & 77.43 & 76.99 & 76.53 & 75.70 \\
\hline Line.113 & 1 & 76.63 & 77.44 & 76.99 & 76.54 & 75.71 \\
\hline Line.113 & 2 & 76.63 & 77.44 & 76.99 & 76.54 & 75.71 \\
\hline Line.114 & 1 & 0.01 & 0.01 & 0.01 & 0.01 & 0.01 \\
\hline Line.114 & 2 & 0.01 & 0.01 & 0.01 & 0.01 & 0.01 \\
\hline Line.115 & 1 & 75.90 & 76.70 & 76.26 & 75.81 & 74.99 \\
\hline Line.115 & 2 & 75.90 & 76.70 & 76.26 & 75.81 & 74.99 \\
\hline Line.116 & 1 & 466.09 & 471.04 & 80.23 & 79.76 & 78.89 \\
\hline
\end{tabular}




\begin{tabular}{|l|c|c|c|c|c|c|}
\hline Element & Terminal & \multicolumn{5}{|c|}{ Faulty Node } \\
\hline & & $\mathbf{7 4 4}$ & $\mathbf{7 2 7}$ & $\mathbf{7 3 0}$ & $\mathbf{7 0 9}$ & $\mathbf{7 0 8}$ \\
\hline Line.116 & 2 & 466.09 & 471.04 & 80.23 & 79.76 & 78.89 \\
\hline Line.117 & 1 & 240.57 & 243.12 & 306.82 & 305.01 & 301.71 \\
\hline Line.117 & 2 & 240.57 & 243.12 & 306.82 & 305.01 & 301.71 \\
\hline Line.118 & 1 & 240.61 & 243.16 & 245.47 & 304.97 & 301.67 \\
\hline Line.118 & 2 & 240.61 & 243.16 & 245.47 & 304.97 & 301.67 \\
\hline Line.119 & 1 & 80.10 & 80.95 & 81.72 & 81.65 & 80.77 \\
\hline Line.119 & 2 & 80.10 & 80.95 & 81.72 & 81.65 & 80.77 \\
\hline Line.120 & 1 & 160.54 & 162.24 & 163.78 & 163.65 & 382.37 \\
\hline Line.120 & 2 & 160.54 & 162.24 & 163.78 & 163.65 & 382.37 \\
\hline Line.121 & 1 & 0.02 & 0.02 & 0.02 & 0.02 & 0.02 \\
\hline Line.121 & 2 & 0.02 & 0.02 & 0.02 & 0.02 & 0.02 \\
\hline Line.122 & 1 & 160.58 & 162.28 & 163.82 & 163.69 & 163.25 \\
\hline Line.122 & 2 & 160.58 & 162.28 & 163.82 & 163.69 & 163.25 \\
\hline Line.123 & 1 & 466.08 & 81.32 & 80.24 & 79.77 & 78.90 \\
\hline Line.123 & 2 & 466.08 & 81.32 & 80.24 & 79.77 & 78.90 \\
\hline Line.124 & 1 & 81.09 & 81.35 & 80.27 & 79.80 & 78.93 \\
\hline Line.124 & 2 & 81.09 & 81.35 & 80.27 & 79.80 & 78.93 \\
\hline Line.125 & 1 & 0.01 & 0.01 & 0.01 & 0.01 & 0.01 \\
\hline Line.125 & 2 & 0.01 & 0.01 & 0.01 & 0.01 & 0.01 \\
\hline Line.126 & 1 & 160.60 & 162.30 & 163.84 & 163.71 & 163.27 \\
\hline Line.126 & 2 & 160.60 & 162.30 & 163.84 & 163.71 & 163.27 \\
\hline Line.127 & 1 & 80.07 & 80.92 & 81.69 & 81.62 & 81.40 \\
\hline Line.127 & 2 & 80.07 & 80.92 & 81.69 & 81.62 & 81.40 \\
\hline Line.128 & 1 & 80.11 & 80.96 & 81.73 & 81.67 & 81.45 \\
\hline Line.128 & 2 & 80.11 & 80.96 & 81.73 & 81.67 & 81.45 \\
\hline Line.129 & 1 & 80.14 & 80.99 & 81.76 & 81.69 & 81.47 \\
\hline Line.129 & 2 & 80.14 & 80.99 & 81.76 & 81.69 & 81.47 \\
\hline Line.130 & 1 & 80.17 & 81.02 & 81.79 & 81.73 & 81.51 \\
\hline Line.130 & 2 & 80.17 & 81.02 & 81.79 & 81.73 & 81.51 \\
\hline Line.131 & 1 & 0.01 & 0.01 & 0.01 & 0.01 & 0.01 \\
\hline Line.131 & 2 & 0.01 & 0.01 & 0.01 & 0.01 & 0.01 \\
\hline Line.132 & 1 & 80.56 & 81.41 & 82.19 & 82.12 & 81.90 \\
\hline Line.132 & 2 & 80.56 & 81.41 & 82.19 & 82.12 & 81.90 \\
\hline Line.133 & 1 & 80.60 & 81.45 & 82.22 & 82.16 & 81.94 \\
\hline Line.133 & 2 & 80.60 & 81.45 & 82.22 & 82.16 & 81.94 \\
\hline Line.134 & 1 & 0.01 & 0.01 & 0.01 & 0.01 & 0.01 \\
\hline Line.134 & 2 & 0.01 & 0.01 & 0.01 & 0.01 & 0.01 \\
\hline
\end{tabular}


Table 4-47: $I_{2}$ value, zone 2, LL fault, $25 \%$ penetration

\begin{tabular}{|c|c|c|c|c|c|c|}
\hline Element & Terminal & \multicolumn{5}{|c|}{ Faulty Node } \\
\hline & & $\mathbf{7 4 4}$ & $\mathbf{7 2 7}$ & $\mathbf{7 3 0}$ & $\mathbf{7 0 9}$ & $\mathbf{7 0 8}$ \\
\hline Vsource & 1 & 6.08 & 6.16 & 6.09 & 6.04 & 5.95 \\
\hline Vsource & 2 & 6.08 & 6.16 & 6.09 & 6.04 & 5.95 \\
\hline Subxf & 1 & 6.08 & 6.16 & 6.09 & 6.04 & 5.95 \\
\hline Subxf & 2 & 291.43 & 295.00 & 291.87 & 289.28 & 285.00 \\
\hline Xfm1 & 1 & 0.00 & 0.00 & 0.00 & 0.00 & 0.00 \\
\hline Xfm1 & 2 & 0.00 & 0.00 & 0.00 & 0.00 & 0.00 \\
\hline Line.11 & 1 & 265.08 & 268.33 & 265.46 & 263.11 & 259.21 \\
\hline Line.11 & 2 & 265.05 & 268.30 & 265.44 & 263.09 & 259.18 \\
\hline Line.12 & 1 & 24.77 & 24.95 & 24.75 & 24.61 & 24.39 \\
\hline Line.12 & 2 & 24.78 & 24.96 & 24.75 & 24.62 & 24.40 \\
\hline Line.13 & 1 & 58.31 & 58.70 & 58.25 & 57.96 & 57.48 \\
\hline Line.13 & 2 & 58.31 & 58.71 & 58.26 & 57.97 & 57.49 \\
\hline Line.14 & 1 & 343.38 & 347.13 & 343.66 & 340.93 & 336.40 \\
\hline Line.14 & 2 & 343.35 & 347.10 & 343.62 & 340.90 & 336.37 \\
\hline Line.15 & 1 & 56.74 & 57.13 & 56.74 & 56.46 & 55.99 \\
\hline Line.15 & 2 & 56.75 & 57.13 & 56.74 & 56.46 & 55.99 \\
\hline Line.16 & 1 & 42.05 & 42.18 & 41.99 & 41.90 & 41.73 \\
\hline Line.16 & 2 & 42.06 & 42.19 & 42.00 & 41.91 & 41.74 \\
\hline Line.17 & 1 & 4.22 & 4.23 & 4.23 & 4.22 & 4.21 \\
\hline Line.17 & 2 & 4.22 & 4.23 & 4.23 & 4.23 & 4.21 \\
\hline Line.18 & 1 & 4.22 & 4.23 & 4.23 & 4.23 & 4.21 \\
\hline Line.18 & 2 & 4.22 & 4.23 & 4.24 & 4.23 & 4.22 \\
\hline Line.19 & 1 & 36.08 & 36.18 & 36.03 & 35.95 & 35.82 \\
\hline Line.19 & 2 & 36.09 & 36.19 & 36.04 & 35.96 & 35.83 \\
\hline Line.110 & 1 & 17.96 & 18.00 & 17.97 & 17.95 & 17.91 \\
\hline Line.110 & 2 & 17.96 & 18.00 & 17.97 & 17.95 & 17.91 \\
\hline Line.111 & 1 & 21.65 & 21.85 & 21.67 & 21.52 & 21.28 \\
\hline Line.111 & 2 & 21.66 & 21.86 & 21.68 & 21.53 & 21.29 \\
\hline Line.112 & 1 & 18.86 & 19.11 & 18.97 & 18.79 & 18.48 \\
\hline Line.112 & 2 & 18.86 & 19.11 & 18.97 & 18.79 & 18.48 \\
\hline Line.113 & 1 & 18.80 & 19.11 & 18.90 & 18.68 & 18.31 \\
\hline Line.113 & 2 & 18.81 & 19.12 & 18.90 & 18.68 & 18.31 \\
\hline Line.114 & 1 & 9.62 & 9.58 & 9.58 & 9.60 & 9.64 \\
\hline Line.114 & 2 & 9.62 & 9.58 & 9.58 & 9.60 & 9.64 \\
\hline Line.115 & 1 & 25.19 & 25.38 & 25.20 & 25.06 & 24.84 \\
\hline Line.115 & 2 & 25.20 & 25.38 & 25.21 & 25.07 & 24.84 \\
\hline Line.116 & 1 & 382.31 & 386.71 & 16.69 & 16.47 & 16.10 \\
\hline
\end{tabular}




\begin{tabular}{|l|c|c|c|c|c|c|}
\hline Element & Terminal & \multicolumn{5}{|c|}{ Faulty Node } \\
\hline & & $\mathbf{7 4 4}$ & $\mathbf{7 2 7}$ & $\mathbf{7 3 0}$ & $\mathbf{7 0 9}$ & $\mathbf{7 0 8}$ \\
\hline Line.116 & 2 & 382.30 & 386.71 & 16.69 & 16.47 & 16.11 \\
\hline Line.117 & 1 & 42.23 & 43.26 & 360.22 & 357.28 & 352.42 \\
\hline Line.117 & 2 & 42.24 & 43.27 & 360.21 & 357.27 & 352.41 \\
\hline Line.118 & 1 & 40.00 & 40.86 & 42.03 & 357.04 & 352.18 \\
\hline Line.118 & 2 & 40.00 & 40.86 & 42.03 & 357.04 & 352.18 \\
\hline Line.119 & 1 & 26.84 & 27.03 & 27.32 & 27.33 & 27.09 \\
\hline Line.119 & 2 & 26.85 & 27.04 & 27.33 & 27.34 & 27.10 \\
\hline Line.120 & 1 & 18.45 & 19.58 & 20.66 & 20.56 & 378.59 \\
\hline Line.120 & 2 & 18.45 & 19.59 & 20.66 & 20.56 & 378.58 \\
\hline Line.121 & 1 & 4.70 & 4.68 & 4.64 & 4.64 & 4.64 \\
\hline Line.121 & 2 & 4.70 & 4.68 & 4.64 & 4.64 & 4.64 \\
\hline Line.122 & 1 & 15.60 & 16.62 & 17.72 & 17.65 & 17.48 \\
\hline Line.122 & 2 & 15.60 & 16.62 & 17.72 & 17.66 & 17.48 \\
\hline Line.123 & 1 & 382.33 & 17.72 & 17.13 & 16.95 & 16.66 \\
\hline Line.123 & 2 & 382.32 & 17.72 & 17.13 & 16.96 & 16.66 \\
\hline Line.124 & 1 & 17.58 & 17.54 & 17.33 & 17.26 & 17.11 \\
\hline Line.124 & 2 & 17.58 & 17.54 & 17.34 & 17.26 & 17.11 \\
\hline Line.125 & 1 & 7.46 & 7.45 & 7.25 & 7.18 & 7.08 \\
\hline Line.125 & 2 & 7.46 & 7.45 & 7.25 & 7.18 & 7.08 \\
\hline Line.126 & 1 & 25.26 & 25.99 & 26.85 & 26.83 & 26.76 \\
\hline Line.126 & 2 & 25.27 & 26.00 & 26.85 & 26.84 & 26.76 \\
\hline Line.127 & 1 & 2.95 & 3.77 & 4.48 & 4.41 & 4.22 \\
\hline Line.127 & 2 & 2.95 & 3.77 & 4.48 & 4.40 & 4.22 \\
\hline Line.128 & 1 & 18.08 & 18.45 & 18.98 & 18.99 & 18.99 \\
\hline Line.128 & 2 & 18.08 & 18.46 & 18.98 & 18.99 & 18.99 \\
\hline Line.129 & 1 & 19.87 & 20.04 & 20.19 & 20.19 & 20.18 \\
\hline Line.129 & 2 & 19.88 & 20.05 & 20.20 & 20.20 & 20.19 \\
\hline Line.130 & 1 & 19.81 & 20.06 & 20.39 & 20.41 & 20.41 \\
\hline Line.130 & 2 & 19.82 & 20.06 & 20.40 & 20.41 & 20.41 \\
\hline Line.131 & 1 & 9.49 & 9.45 & 9.36 & 9.36 & 9.36 \\
\hline Line.131 & 2 & 9.49 & 9.44 & 9.36 & 9.35 & 9.35 \\
\hline Line.132 & 1 & 22.90 & 23.10 & 23.34 & 23.35 & 23.35 \\
\hline Line.132 & 2 & 22.91 & 23.11 & 23.35 & 23.36 & 23.36 \\
\hline Line.133 & 1 & 24.20 & 24.42 & 24.76 & 24.77 & 24.78 \\
\hline Line.133 & 2 & 24.22 & 24.44 & 24.78 & 24.79 & 24.80 \\
\hline Line.134 & 1 & 9.50 & 9.46 & 9.37 & 9.36 & 9.36 \\
\hline Line.134 & 2 & 9.50 & 9.45 & 9.37 & 9.36 & 9.36 \\
\hline
\end{tabular}


Table 4-48: $I_{1}$ value, zone 3, 3 phase fault, $100 \%$ penetration

\begin{tabular}{|c|c|c|c|c|c|c|}
\hline Element & Terminal & \multicolumn{5}{|c|}{ Faulty Node } \\
\hline & & $\mathbf{7 3 4}$ & $\mathbf{7 1 0}$ & $\mathbf{7 3 7}$ & $\mathbf{7 3 8}$ & $\mathbf{7 1 1}$ \\
\hline Vsource & 1 & 13.95 & 13.64 & 13.67 & 13.52 & 13.36 \\
\hline Vsource & 2 & 13.95 & 13.64 & 13.67 & 13.52 & 13.36 \\
\hline Subxf & 1 & 13.95 & 13.64 & 13.67 & 13.52 & 13.36 \\
\hline Subxf & 2 & 668.28 & 653.44 & 655.24 & 647.63 & 640.25 \\
\hline Xfm1 & 1 & 0.00 & 0.00 & 0.00 & 0.00 & 0.00 \\
\hline Xfm1 & 2 & 0.00 & 0.00 & 0.00 & 0.00 & 0.00 \\
\hline Line.11 & 1 & 605.52 & 591.76 & 593.49 & 586.48 & 579.68 \\
\hline Line.11 & 2 & 605.52 & 591.76 & 593.50 & 586.48 & 579.68 \\
\hline Line.12 & 1 & 22.27 & 21.01 & 21.32 & 20.77 & 20.23 \\
\hline Line.12 & 2 & 22.27 & 21.01 & 21.32 & 20.76 & 20.23 \\
\hline Line.13 & 1 & 28.17 & 26.04 & 27.00 & 26.32 & 25.68 \\
\hline Line.13 & 2 & 28.17 & 26.04 & 27.00 & 26.32 & 25.67 \\
\hline Line.14 & 1 & 651.19 & 633.65 & 636.38 & 627.73 & 619.34 \\
\hline Line.14 & 2 & 651.20 & 633.65 & 636.39 & 627.73 & 619.35 \\
\hline Line.15 & 1 & 30.04 & 28.08 & 28.89 & 28.21 & 27.56 \\
\hline Line.15 & 2 & 30.04 & 28.08 & 28.88 & 28.21 & 27.55 \\
\hline Line.16 & 1 & 11.80 & 11.26 & 11.84 & 11.88 & 11.92 \\
\hline Line.16 & 2 & 11.79 & 11.25 & 11.83 & 11.87 & 11.91 \\
\hline Line.17 & 1 & 1.28 & 1.46 & 1.40 & 1.47 & 1.53 \\
\hline Line.17 & 2 & 1.28 & 1.46 & 1.40 & 1.47 & 1.53 \\
\hline Line.18 & 1 & 1.28 & 1.46 & 1.40 & 1.47 & 1.53 \\
\hline Line.18 & 2 & 1.28 & 1.46 & 1.40 & 1.47 & 1.53 \\
\hline Line.19 & 1 & 13.40 & 12.80 & 13.23 & 13.12 & 13.01 \\
\hline Line.19 & 2 & 13.40 & 12.80 & 13.22 & 13.12 & 13.00 \\
\hline Line.110 & 1 & 19.36 & 19.36 & 19.36 & 19.36 & 19.36 \\
\hline Line.110 & 2 & 19.36 & 19.36 & 19.36 & 19.36 & 19.36 \\
\hline Line.111 & 1 & 29.03 & 28.25 & 28.37 & 27.98 & 27.61 \\
\hline Line.111 & 2 & 29.03 & 28.25 & 28.37 & 27.98 & 27.61 \\
\hline Line.112 & 1 & 20.92 & 20.01 & 20.20 & 19.78 & 19.37 \\
\hline Line.112 & 2 & 20.92 & 20.01 & 20.20 & 19.78 & 19.37 \\
\hline Line.113 & 1 & 25.16 & 24.25 & 24.40 & 23.96 & 23.53 \\
\hline Line.113 & 2 & 25.16 & 24.25 & 24.40 & 23.96 & 23.53 \\
\hline Line.114 & 1 & 2.67 & 3.04 & 2.92 & 3.07 & 3.21 \\
\hline Line.114 & 2 & 2.67 & 3.04 & 2.92 & 3.07 & 3.21 \\
\hline Line.115 & 1 & 24.72 & 23.79 & 23.96 & 23.52 & 23.09 \\
\hline Line.115 & 2 & 24.72 & 23.79 & 23.96 & 23.52 & 23.09 \\
\hline Line.116 & 1 & 18.79 & 17.35 & 17.79 & 17.19 & 16.62 \\
\hline
\end{tabular}




\begin{tabular}{|l|c|c|c|c|c|c|}
\hline Element & Terminal & \multicolumn{5}{|c|}{ Faulty Node } \\
\hline & & $\mathbf{7 3 4}$ & $\mathbf{7 1 0}$ & $\mathbf{7 3 7}$ & $\mathbf{7 3 8}$ & $\mathbf{7 1 1}$ \\
\hline Line.116 & 2 & 18.79 & 17.35 & 17.79 & 17.19 & 16.62 \\
\hline Line.117 & 1 & 669.32 & 650.28 & 653.40 & 644.09 & 635.08 \\
\hline Line.117 & 2 & 669.32 & 650.28 & 653.40 & 644.10 & 635.08 \\
\hline Line.118 & 1 & 667.44 & 648.07 & 651.32 & 641.88 & 632.75 \\
\hline Line.118 & 2 & 667.44 & 648.07 & 651.32 & 641.88 & 632.75 \\
\hline Line.119 & 1 & 32.19 & 31.14 & 31.34 & 30.84 & 30.37 \\
\hline Line.119 & 2 & 32.18 & 31.14 & 31.34 & 30.84 & 30.36 \\
\hline Line.120 & 1 & 698.96 & 678.57 & 682.01 & 672.09 & 662.49 \\
\hline Line.120 & 2 & 698.96 & 678.57 & 682.01 & 672.09 & 662.49 \\
\hline Line.121 & 1 & 0.92 & 1.11 & 1.05 & 1.13 & 1.20 \\
\hline Line.121 & 2 & 0.92 & 1.11 & 1.05 & 1.13 & 1.20 \\
\hline Line.122 & 1 & 698.05 & 677.46 & 680.98 & 670.99 & 661.32 \\
\hline Line.122 & 2 & 698.05 & 677.46 & 680.98 & 670.99 & 661.32 \\
\hline Line.123 & 1 & 19.85 & 18.57 & 18.92 & 18.37 & 17.84 \\
\hline Line.123 & 2 & 19.85 & 18.56 & 18.92 & 18.37 & 17.84 \\
\hline Line.124 & 1 & 24.28 & 23.65 & 23.72 & 23.39 & 23.07 \\
\hline Line.124 & 2 & 24.28 & 23.65 & 23.72 & 23.39 & 23.07 \\
\hline Line.125 & 1 & 3.56 & 4.12 & 3.93 & 4.15 & 4.37 \\
\hline Line.125 & 2 & 3.56 & 4.12 & 3.93 & 4.15 & 4.37 \\
\hline Line.126 & 1 & 687.85 & 667.31 & 670.85 & 660.91 & 651.27 \\
\hline Line.126 & 2 & 687.85 & 667.31 & 670.85 & 660.91 & 651.28 \\
\hline Line.127 & 1 & 11.64 & 11.48 & 698.10 & 687.51 & 677.25 \\
\hline Line.127 & 2 & 11.63 & 11.47 & 698.10 & 687.51 & 677.25 \\
\hline Line.128 & 1 & 26.88 & 25.36 & 26.99 & 670.70 & 660.50 \\
\hline Line.128 & 2 & 26.88 & 25.36 & 26.99 & 670.70 & 660.50 \\
\hline Line.129 & 1 & 28.98 & 27.95 & 29.04 & 29.08 & 658.18 \\
\hline Line.129 & 2 & 28.98 & 27.95 & 29.04 & 29.08 & 658.18 \\
\hline Line.130 & 1 & 30.44 & 29.75 & 30.44 & 30.45 & 30.46 \\
\hline Line.130 & 2 & 30.44 & 29.75 & 30.44 & 30.45 & 30.46 \\
\hline Line.131 & 1 & 1.49 & 1.88 & 1.44 & 1.41 & 1.39 \\
\hline Line.131 & 2 & 1.49 & 1.88 & 1.44 & 1.41 & 1.39 \\
\hline Line.132 & 1 & 29.50 & 673.76 & 28.51 & 27.92 & 27.36 \\
\hline Line.132 & 2 & 29.50 & 673.76 & 28.50 & 27.92 & 27.36 \\
\hline Line.133 & 1 & 30.97 & 30.95 & 30.19 & 29.74 & 29.30 \\
\hline Line.133 & 2 & 30.97 & 30.95 & 30.19 & 29.74 & 29.30 \\
\hline Line.134 & 1 & 1.49 & 1.42 & 1.74 & 1.89 & 2.05 \\
\hline Line.134 & 2 & 1.49 & 1.42 & 1.74 & 1.89 & 2.05 \\
\hline
\end{tabular}


Table 4-49: $I_{r}$ value, zone 3, SLG fault, $25 \%$ penetration

\begin{tabular}{|c|c|c|c|c|c|c|}
\hline Element & Terminal & \multicolumn{5}{|c|}{ Faulty Node } \\
\hline & & $\mathbf{7 3 4}$ & $\mathbf{7 1 0}$ & $\mathbf{7 3 7}$ & $\mathbf{7 3 8}$ & $\mathbf{7 1 1}$ \\
\hline Vsource & 1 & 0.00 & 0.00 & 0.00 & 0.00 & 0.00 \\
\hline Vsource & 2 & 0.00 & 0.00 & 0.00 & 0.00 & 0.00 \\
\hline Subxf & 1 & 0.00 & 0.00 & 0.00 & 0.00 & 0.00 \\
\hline Subxf & 2 & 0.00 & 0.00 & 0.00 & 0.00 & 0.00 \\
\hline Xfm1 & 1 & 0.00 & 0.00 & 0.00 & 0.00 & 0.00 \\
\hline Xfm1 & 2 & 0.00 & 0.00 & 0.00 & 0.00 & 0.00 \\
\hline Line.11 & 1 & 0.24 & 0.23 & 0.24 & 0.23 & 0.23 \\
\hline Line.11 & 2 & 0.24 & 0.23 & 0.24 & 0.23 & 0.23 \\
\hline Line.12 & 1 & 72.75 & 70.82 & 71.01 & 69.94 & 68.87 \\
\hline Line.12 & 2 & 72.75 & 70.82 & 71.01 & 69.94 & 68.87 \\
\hline Line.13 & 1 & 144.00 & 140.18 & 140.57 & 138.44 & 136.33 \\
\hline Line.13 & 2 & 144.00 & 140.18 & 140.57 & 138.44 & 136.33 \\
\hline Line.14 & 1 & 216.40 & 210.66 & 211.24 & 208.05 & 204.88 \\
\hline Line.14 & 2 & 216.40 & 210.66 & 211.24 & 208.05 & 204.88 \\
\hline Line.15 & 1 & 144.03 & 140.20 & 140.59 & 138.46 & 136.36 \\
\hline Line.15 & 2 & 144.03 & 140.20 & 140.59 & 138.46 & 136.36 \\
\hline Line.16 & 1 & 70.59 & 68.72 & 68.91 & 67.86 & 66.83 \\
\hline Line.16 & 2 & 70.59 & 68.72 & 68.91 & 67.86 & 66.83 \\
\hline Line.17 & 1 & 0.05 & 0.05 & 0.05 & 0.05 & 0.05 \\
\hline Line.17 & 2 & 0.05 & 0.05 & 0.05 & 0.05 & 0.05 \\
\hline Line.18 & 1 & 0.01 & 0.01 & 0.01 & 0.01 & 0.01 \\
\hline Line.18 & 2 & 0.01 & 0.01 & 0.01 & 0.01 & 0.01 \\
\hline Line.19 & 1 & 70.69 & 68.81 & 69.00 & 67.96 & 66.92 \\
\hline Line.19 & 2 & 70.69 & 68.81 & 69.00 & 67.96 & 66.92 \\
\hline Line.110 & 1 & 0.01 & 0.01 & 0.01 & 0.01 & 0.01 \\
\hline Line.110 & 2 & 0.01 & 0.01 & 0.01 & 0.01 & 0.01 \\
\hline Line.111 & 1 & 70.74 & 68.86 & 69.05 & 68.01 & 66.97 \\
\hline Line.111 & 2 & 70.74 & 68.86 & 69.05 & 68.01 & 66.97 \\
\hline Line.112 & 1 & 73.47 & 71.52 & 71.72 & 70.63 & 69.56 \\
\hline Line.112 & 2 & 73.47 & 71.52 & 71.72 & 70.63 & 69.56 \\
\hline Line.113 & 1 & 73.48 & 71.53 & 71.72 & 70.64 & 69.56 \\
\hline Line.113 & 2 & 73.48 & 71.53 & 71.72 & 70.64 & 69.56 \\
\hline Line.114 & 1 & 0.01 & 0.01 & 0.01 & 0.01 & 0.01 \\
\hline Line.114 & 2 & 0.01 & 0.01 & 0.01 & 0.01 & 0.01 \\
\hline Line.115 & 1 & 72.78 & 70.85 & 71.04 & 69.97 & 68.90 \\
\hline Line.115 & 2 & 72.78 & 70.85 & 71.04 & 69.97 & 68.90 \\
\hline Line.116 & 1 & 76.57 & 74.54 & 74.74 & 73.61 & 72.49 \\
\hline
\end{tabular}




\begin{tabular}{|l|c|c|c|c|c|c|}
\hline Element & Terminal & \multicolumn{5}{|c|}{ Faulty Node } \\
\hline & & $\mathbf{7 3 4}$ & $\mathbf{7 1 0}$ & $\mathbf{7 3 7}$ & $\mathbf{7 3 8}$ & $\mathbf{7 1 1}$ \\
\hline Line.116 & 2 & 76.57 & 74.54 & 74.74 & 73.61 & 72.49 \\
\hline Line.117 & 1 & 292.82 & 285.05 & 285.84 & 281.51 & 277.22 \\
\hline Line.117 & 2 & 292.82 & 285.05 & 285.84 & 281.51 & 277.22 \\
\hline Line.118 & 1 & 292.78 & 285.01 & 285.80 & 281.48 & 277.19 \\
\hline Line.118 & 2 & 292.78 & 285.01 & 285.80 & 281.48 & 277.19 \\
\hline Line.119 & 1 & 78.39 & 76.31 & 76.52 & 75.36 & 74.21 \\
\hline Line.119 & 2 & 78.39 & 76.31 & 76.52 & 75.36 & 74.21 \\
\hline Line.120 & 1 & 371.11 & 361.26 & 362.26 & 356.78 & 351.34 \\
\hline Line.120 & 2 & 371.11 & 361.26 & 362.26 & 356.78 & 351.34 \\
\hline Line.121 & 1 & 0.02 & 0.02 & 0.02 & 0.02 & 0.02 \\
\hline Line.121 & 2 & 0.02 & 0.02 & 0.02 & 0.02 & 0.02 \\
\hline Line.122 & 1 & 371.07 & 361.22 & 362.22 & 356.74 & 351.31 \\
\hline Line.122 & 2 & 371.07 & 361.22 & 362.22 & 356.74 & 351.31 \\
\hline Line.123 & 1 & 76.58 & 74.55 & 74.75 & 73.62 & 72.50 \\
\hline Line.123 & 2 & 76.58 & 74.55 & 74.75 & 73.62 & 72.50 \\
\hline Line.124 & 1 & 76.61 & 74.57 & 74.78 & 73.65 & 72.53 \\
\hline Line.124 & 2 & 76.61 & 74.57 & 74.78 & 73.65 & 72.53 \\
\hline Line.125 & 1 & 0.01 & 0.01 & 0.01 & 0.01 & 0.01 \\
\hline Line.125 & 2 & 0.01 & 0.01 & 0.01 & 0.01 & 0.01 \\
\hline Line.126 & 1 & 371.05 & 361.21 & 362.21 & 356.72 & 351.29 \\
\hline Line.126 & 2 & 371.05 & 361.21 & 362.21 & 356.72 & 351.29 \\
\hline Line.127 & 1 & 80.84 & 78.69 & 441.45 & 434.76 & 428.14 \\
\hline Line.127 & 2 & 80.84 & 78.69 & 441.45 & 434.76 & 428.14 \\
\hline Line.128 & 1 & 80.88 & 78.73 & 80.31 & 434.72 & 428.10 \\
\hline Line.128 & 2 & 80.88 & 78.73 & 80.31 & 434.72 & 428.10 \\
\hline Line.129 & 1 & 80.91 & 78.76 & 80.34 & 79.99 & 428.08 \\
\hline Line.129 & 2 & 80.91 & 78.76 & 80.34 & 79.99 & 428.08 \\
\hline Line.130 & 1 & 80.94 & 78.79 & 80.37 & 80.02 & 79.68 \\
\hline Line.130 & 2 & 80.94 & 78.79 & 80.37 & 80.02 & 79.68 \\
\hline Line.131 & 1 & 0.01 & 0.01 & 0.01 & 0.01 & 0.01 \\
\hline Line.131 & 2 & 0.01 & 0.01 & 0.01 & 0.01 & 0.01 \\
\hline Line.132 & 1 & 81.33 & 439.73 & 79.39 & 78.19 & 77.00 \\
\hline Line.132 & 2 & 81.33 & 439.73 & 79.39 & 78.19 & 77.00 \\
\hline Line.133 & 1 & 81.37 & 80.59 & 79.43 & 78.22 & 77.03 \\
\hline Line.133 & 2 & 81.37 & 80.59 & 79.43 & 78.22 & 77.03 \\
\hline Line.134 & 1 & 0.01 & 0.01 & 0.01 & 0.01 & 0.01 \\
\hline Line.134 & 2 & 0.01 & 0.01 & 0.01 & 0.01 & 0.01 \\
\hline
\end{tabular}


Table 4-50: $I_{2}$ value, zone 3 , LL fault, $25 \%$ penetration

\begin{tabular}{|c|c|c|c|c|c|c|}
\hline Element & Terminal & \multicolumn{5}{|c|}{ Faulty Node } \\
\hline & & $\mathbf{7 3 4}$ & $\mathbf{7 1 0}$ & $\mathbf{7 3 7}$ & $\mathbf{7 3 8}$ & $\mathbf{7 1 1}$ \\
\hline Vsource & 1 & 5.72 & 5.52 & 5.56 & 5.46 & 5.38 \\
\hline Vsource & 2 & 5.72 & 5.52 & 5.56 & 5.46 & 5.38 \\
\hline Subxf & 1 & 5.72 & 5.52 & 5.56 & 5.46 & 5.38 \\
\hline Subxf & 2 & 273.97 & 264.73 & 266.25 & 261.85 & 257.58 \\
\hline Xfm1 & 1 & 0.00 & 0.00 & 0.00 & 0.00 & 0.00 \\
\hline Xfm1 & 2 & 0.00 & 0.00 & 0.00 & 0.00 & 0.00 \\
\hline Line.11 & 1 & 249.17 & 240.77 & 242.13 & 238.13 & 234.24 \\
\hline Line.11 & 2 & 249.14 & 240.75 & 242.11 & 238.11 & 234.22 \\
\hline Line.12 & 1 & 23.83 & 23.43 & 23.44 & 23.21 & 23.00 \\
\hline Line.12 & 2 & 23.83 & 23.43 & 23.44 & 23.22 & 23.00 \\
\hline Line.13 & 1 & 56.22 & 55.27 & 55.31 & 54.79 & 54.27 \\
\hline Line.13 & 2 & 56.22 & 55.27 & 55.31 & 54.79 & 54.28 \\
\hline Line.14 & 1 & 324.72 & 315.18 & 316.52 & 311.84 & 307.30 \\
\hline Line.14 & 2 & 324.69 & 315.15 & 316.49 & 311.81 & 307.27 \\
\hline Line.15 & 1 & 54.75 & 53.75 & 53.87 & 53.36 & 52.85 \\
\hline Line.15 & 2 & 54.76 & 53.76 & 53.87 & 53.36 & 52.86 \\
\hline Line.16 & 1 & 41.29 & 40.98 & 40.96 & 40.77 & 40.58 \\
\hline Line.16 & 2 & 41.30 & 40.99 & 40.97 & 40.78 & 40.58 \\
\hline Line.17 & 1 & 4.18 & 4.15 & 4.17 & 4.17 & 4.16 \\
\hline Line.17 & 2 & 4.19 & 4.16 & 4.18 & 4.17 & 4.17 \\
\hline Line.18 & 1 & 4.19 & 4.16 & 4.18 & 4.17 & 4.17 \\
\hline Line.18 & 2 & 4.19 & 4.16 & 4.18 & 4.18 & 4.17 \\
\hline Line.19 & 1 & 35.47 & 35.23 & 35.21 & 35.06 & 34.90 \\
\hline Line.19 & 2 & 35.48 & 35.24 & 35.22 & 35.07 & 34.91 \\
\hline Line.110 & 1 & 17.81 & 17.73 & 17.74 & 17.70 & 17.66 \\
\hline Line.110 & 2 & 17.82 & 17.73 & 17.75 & 17.71 & 17.67 \\
\hline Line.111 & 1 & 20.65 & 20.14 & 20.22 & 19.97 & 19.72 \\
\hline Line.111 & 2 & 20.66 & 20.15 & 20.22 & 19.97 & 19.73 \\
\hline Line.112 & 1 & 17.67 & 16.86 & 17.11 & 16.78 & 16.46 \\
\hline Line.112 & 2 & 17.67 & 16.86 & 17.11 & 16.78 & 16.46 \\
\hline Line.113 & 1 & 17.36 & 16.48 & 16.71 & 16.33 & 15.97 \\
\hline Line.113 & 2 & 17.37 & 16.49 & 16.71 & 16.34 & 15.98 \\
\hline Line.114 & 1 & 9.75 & 9.88 & 9.82 & 9.86 & 9.90 \\
\hline Line.114 & 2 & 9.75 & 9.88 & 9.82 & 9.86 & 9.90 \\
\hline Line.115 & 1 & 24.25 & 23.76 & 23.84 & 23.61 & 23.38 \\
\hline Line.115 & 2 & 24.25 & 23.77 & 23.84 & 23.61 & 23.38 \\
\hline & & & & & & \\
\hline
\end{tabular}




\begin{tabular}{|l|c|c|c|c|c|c|}
\hline Element & Terminal & \multicolumn{5}{|c|}{ Faulty Node } \\
\hline & & $\mathbf{7 3 4}$ & $\mathbf{7 1 0}$ & $\mathbf{7 3 7}$ & $\mathbf{7 3 8}$ & $\mathbf{7 1 1}$ \\
\hline Line.116 & 1 & 15.17 & 14.33 & 14.51 & 14.13 & 13.76 \\
\hline Line.116 & 2 & 15.18 & 14.33 & 14.52 & 14.14 & 13.76 \\
\hline Line.117 & 1 & 339.83 & 329.44 & 330.97 & 325.92 & 321.01 \\
\hline Line.117 & 2 & 339.82 & 329.44 & 330.97 & 325.92 & 321.00 \\
\hline Line.118 & 1 & 339.62 & 329.34 & 330.78 & 325.74 & 320.84 \\
\hline Line.118 & 2 & 339.61 & 329.34 & 330.77 & 325.74 & 320.84 \\
\hline Line.119 & 1 & 26.45 & 25.94 & 26.01 & 25.76 & 25.51 \\
\hline Line.119 & 2 & 26.46 & 25.95 & 26.02 & 25.77 & 25.52 \\
\hline Line.120 & 1 & 365.36 & 354.53 & 356.06 & 350.76 & 345.60 \\
\hline Line.120 & 2 & 365.36 & 354.52 & 356.06 & 350.76 & 345.60 \\
\hline Line.121 & 1 & 4.69 & 4.76 & 4.73 & 4.76 & 4.78 \\
\hline Line.121 & 2 & 4.69 & 4.76 & 4.73 & 4.75 & 4.78 \\
\hline Line.122 & 1 & 365.32 & 354.54 & 356.03 & 350.73 & 345.58 \\
\hline Line.122 & 2 & 365.32 & 354.54 & 356.02 & 350.73 & 345.58 \\
\hline Line.123 & 1 & 15.85 & 14.98 & 15.26 & 14.91 & 14.57 \\
\hline Line.123 & 2 & 15.85 & 14.98 & 15.26 & 14.92 & 14.58 \\
\hline Line.124 & 1 & 16.61 & 15.96 & 16.20 & 15.95 & 15.70 \\
\hline Line.124 & 2 & 16.61 & 15.96 & 16.21 & 15.96 & 15.71 \\
\hline Line.125 & 1 & 6.80 & 6.57 & 6.61 & 6.50 & 6.40 \\
\hline Line.125 & 2 & 6.80 & 6.57 & 6.61 & 6.50 & 6.40 \\
\hline Line.126 & 1 & 355.22 & 344.53 & 346.11 & 340.91 & 335.83 \\
\hline Line.126 & 2 & 355.21 & 344.52 & 346.10 & 340.90 & 335.82 \\
\hline Line.127 & 1 & 3.86 & 1.99 & 369.07 & 363.57 & 358.21 \\
\hline Line.127 & 2 & 3.86 & 1.99 & 369.06 & 363.56 & 358.21 \\
\hline Line.128 & 1 & 19.02 & 17.98 & 19.04 & 346.83 & 341.63 \\
\hline Line.128 & 2 & 19.03 & 17.98 & 19.04 & 346.82 & 341.63 \\
\hline Line.129 & 1 & 20.17 & 19.77 & 20.16 & 20.18 & 340.28 \\
\hline Line.129 & 2 & 20.18 & 19.78 & 20.17 & 20.19 & 340.27 \\
\hline Line.130 & 1 & 20.44 & 19.77 & 20.46 & 20.50 & 20.54 \\
\hline Line.130 & 2 & 20.45 & 19.77 & 20.47 & 20.51 & 20.54 \\
\hline Line.131 & 1 & 9.35 & 9.50 & 9.36 & 9.36 & 9.36 \\
\hline Line.131 & 2 & 9.35 & 9.50 & 9.36 & 9.36 & 9.36 \\
\hline Line.132 & 1 & 23.37 & 345.54 & 22.86 & 22.57 & 22.29 \\
\hline Line.132 & 2 & 23.38 & 345.53 & 22.86 & 22.58 & 22.30 \\
\hline Line.133 & 1 & 24.84 & 24.90 & 24.29 & 23.98 & 23.68 \\
\hline Line.133 & 2 & 24.85 & 24.92 & 24.31 & 24.00 & 23.70 \\
\hline Line.134 & 1 & 9.36 & 9.36 & 9.45 & 9.49 & 9.54 \\
\hline Line.134 & 2 & 9.36 & 9.36 & 9.45 & 9.49 & 9.54 \\
\hline
\end{tabular}




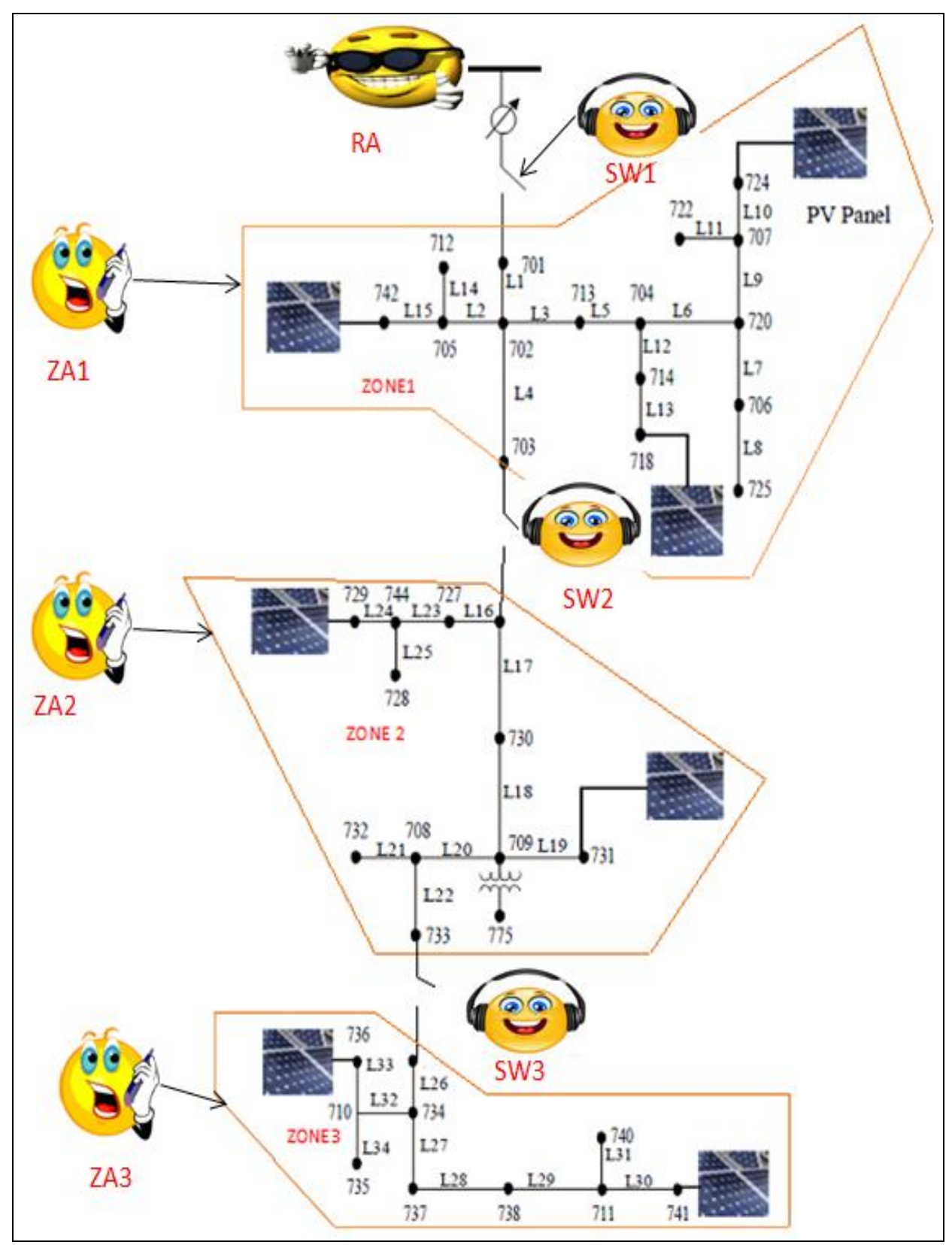

Figure 4-6: Test System with Agents 


\subsection{Fault Location Test Cases}

The fault location algorithm discussed in section 4.3, is embedded in the Switch Agents. These Switch Agents only act after receiving the recloser lock out signal from its Zone Agent, which in turn receives the signal from the Recloser Agent.

The first step in the simulation process is to create the fault at a particular node in the OpenDSS software. Once the fault simulation is done, the power flow results, along with the values of sequence currents with their phase angles for all the lines in the system at an instant before opening the substation transformer, are written to an Excel file.

In order to mimic the locking out action of the recloser, the substation transformer is opened after the fault simulation. The Recloser Agent, then seeing no current in the substation transformer, will inform each of the Zone Agents about the lock out. In turn the Zone Agents inform their respective Switch Agents to implement the fault location algorithm.

The algorithm for fault location has been implemented for different penetration levels of Distributed Generation like 25\%, 50\%, 75\% and 100\%. The only difference observed between these penetration levels is the change in the threshold values of the sequence current magnitudes, while remainder of the observations discussed in the algorithm for fault location in section 4.3 remain valid. In the following section, the various test cases for the fault location for $50 \%$ penetration level are presented.

\subsubsection{Single line to ground Fault at node 713}

A SLG fault is simulated in OpenDSS, then the power flow results along with values of sequence currents of all the lines along with their phase angles at the instant before opening substation transformer are written to an Excel file. The Recloser Agent informs its locking out action to all the Zonal Agents ZA1, ZA2 \&ZA3, which in turn pass the message to their corresponding Switch Agents SW1, SW2\& SW3. Then, each of these agents starts reading the sequence current magnitudes of the lines in their zone. For a SLG fault in zone $1 \mathrm{I}_{\mathrm{r}}$ th is $650 \mathrm{~A}$ (Table 4-23) and the values of $\mathrm{I}_{\mathrm{r}}$ in all the lines in the test system for the SLG fault at node 713 (zone1) can be seen in the Table 4-15. SW1, upon reading the values of Ir from the Excel file, observes that threshold of $650 \mathrm{~A}$ for $I_{r}$ is 
violated by the line L3. The current reversal is determined by looking at the phase angle of the zero sequence current (Io). If the fault exists at a node which has DG unit feeding it on either side, then the phase angles of Io for the adjoining lines will be opposite. The Switch Agent, after checking this condition for current reversal, observes the current reversal between lines L3 and L5. Hence, the faulty node would be 713. The plot of the Io phase angles for the lines L3 and L5 shown in the Figure4-7 confirms the current reversal. The fault is simulated at 0.007 seconds.

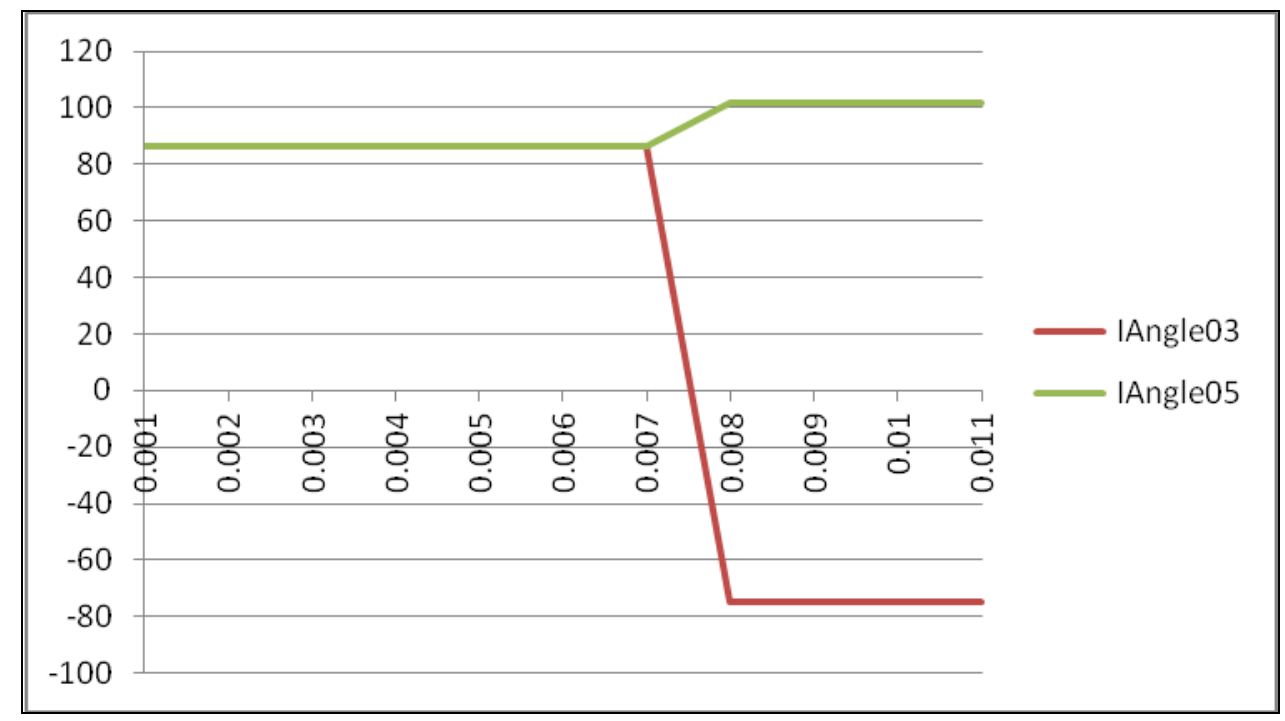

Figure 4-7: I0 phase angle reversal for fault at $\mathbf{7 1 3}$

The output screen of the JADE output in the command prompt is shown in the figure below:

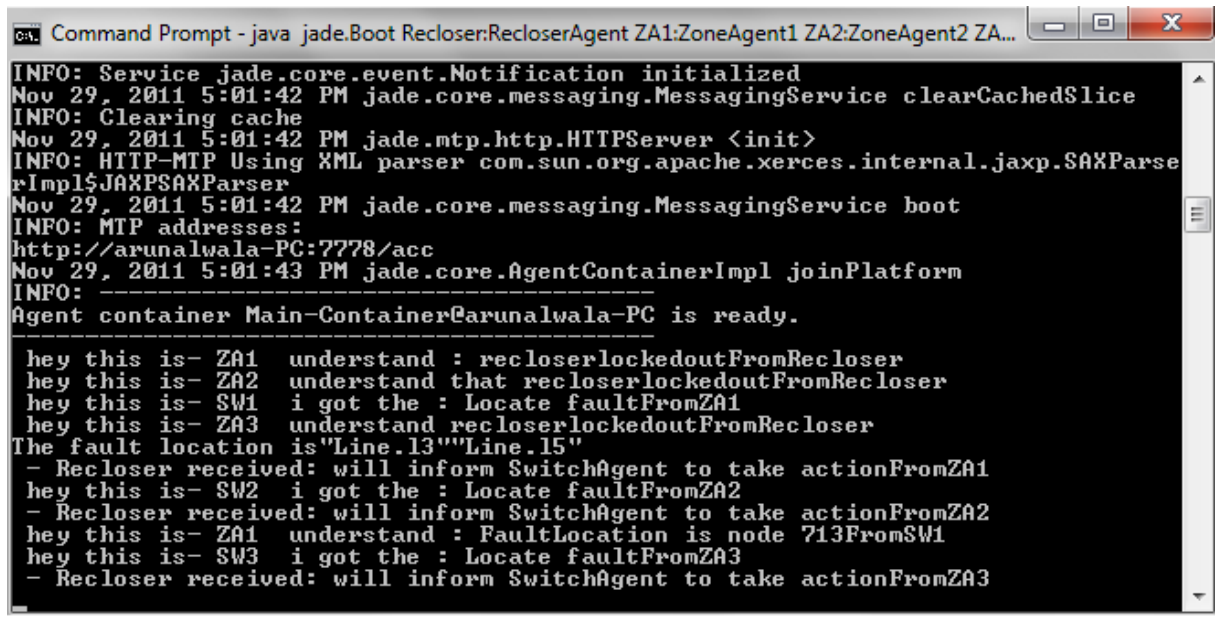

Figure 4-8: JADE Output for fault at 713 Node 


\subsubsection{Single line to ground Fault at node 706:}

Upon receiving the lock out signal from their respective Zone Agents, the Switch Agents start looking for threshold limit violations in the magnitude of sequence current components in all the distribution lines in their zone. For a SLG fault in zone1, $\mathrm{I}_{\mathrm{r}}$ th is 650A (Table 4-23) and the values of $I_{r}$ in all the lines in the test system for the SLG fault at 706 (zone1) can be seen from the Table 4-15. Since this is a SLG fault in Zone 1, SwitchAgent1 sees a violation in $\mathrm{I}_{\mathrm{r}}$ th in the lines L6 and L7. First, the adjoining lines of L6 and L7 are checked for current reversal and these lines do not satisfy this condition. But, the agent observes that the line L8, which is located after L7, has a low value of residual current and decides that the fault exists between the lines L7 and L8, that is at 706. The output screen of the JADE output in the command prompt is shown in the Figure 4-8 shown below:

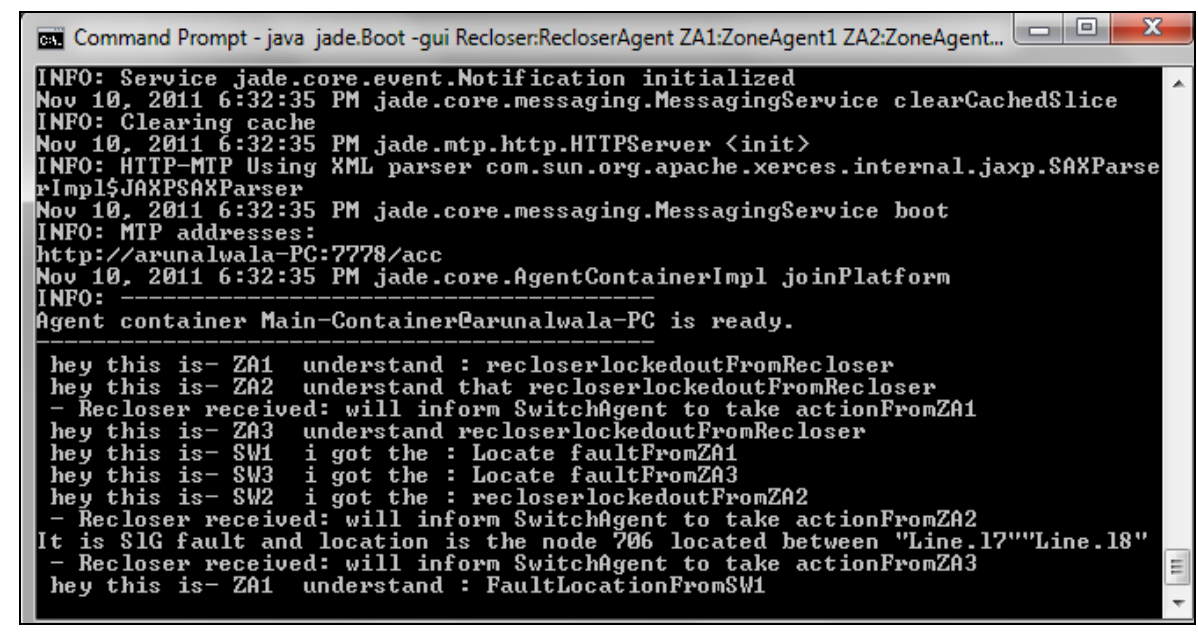

Figure 4-9: JADE Output for fault at 706 Node

The agent message passing for fault location in zone1 is represented from sniffer agent GUI shown in the Figure 4-9. 


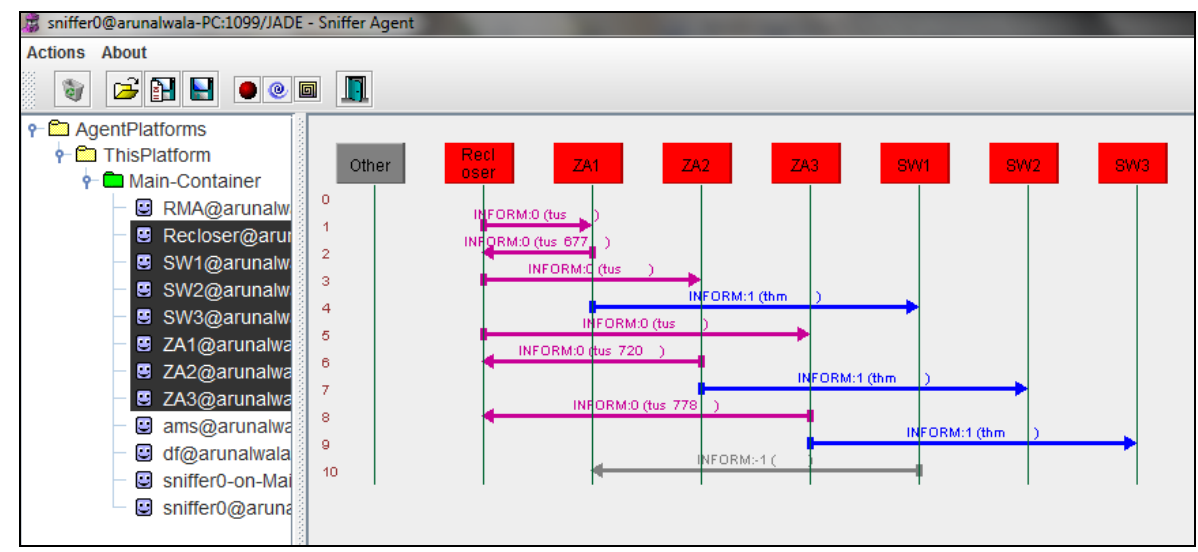

Figure 4-10: Agent Message exchange for Fault Location in Zone1

\subsubsection{Three phase to ground fault at node 730}

In this test case, a 3LG fault is simulated in zone2. So, one of the Switch Agents will find a violation in $\mathrm{I}_{1}$ th. The Switch Agents in all the zones start checking sequence current magnitudes and SW2 notices a violation in $\mathrm{I}_{1}$ thof L17. For a $3 \mathrm{LG}$ fault in zone2, $\mathrm{I}_{1}$ th is 900A (Table 4-23) and the values of $I_{1}$ in all the lines in the test system for the $3 \mathrm{LG}$ fault at node 730 (zone2) can be seen from Table 4-17.The current reversal for three phase fault is determined by checking direction of real power in all the 3 phases of the distribution line. When there is a faulty node with generation sources on either side of it, the real power in all the three phases of the adjoining lines of it will be opposite, that is the real power will be flowing towards the faulty node. The power flow in all the lines after the fault is shown in Table 4-51. The power flow results show that the real power in all the three phases in the lines L17 and L18 are in the opposite direction and flowing towards the node 730 . Hence, the faulty node is 730 . The power flow in zone2 after the fault at 730 is depicted in the Figure4-13. Also, the change in the phase angles of the positive sequence current of the lines L17 and L18 is opposite. The plot of the $\mathrm{I}_{1}$ phase angles for the lines L17 and L18 shown in the Figure 4-12, confirms the current reversal as well. The fault is simulated at 0.007 seconds. The JADE output for this case is shown in the figure below: 


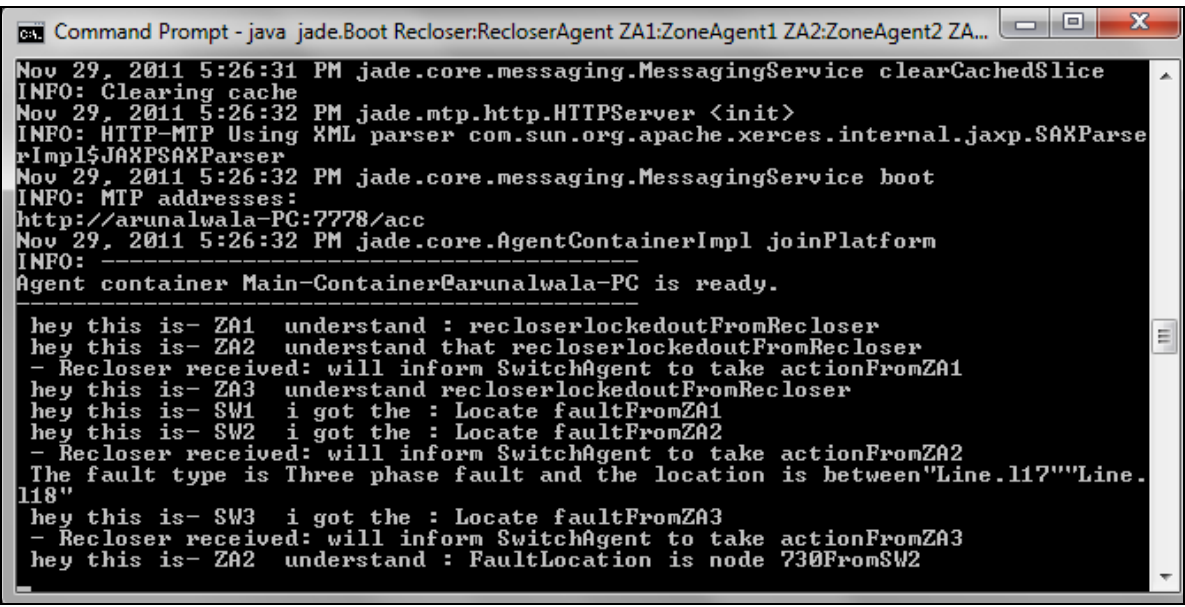

Figure 4-11: JADE Output for fault at 730 node

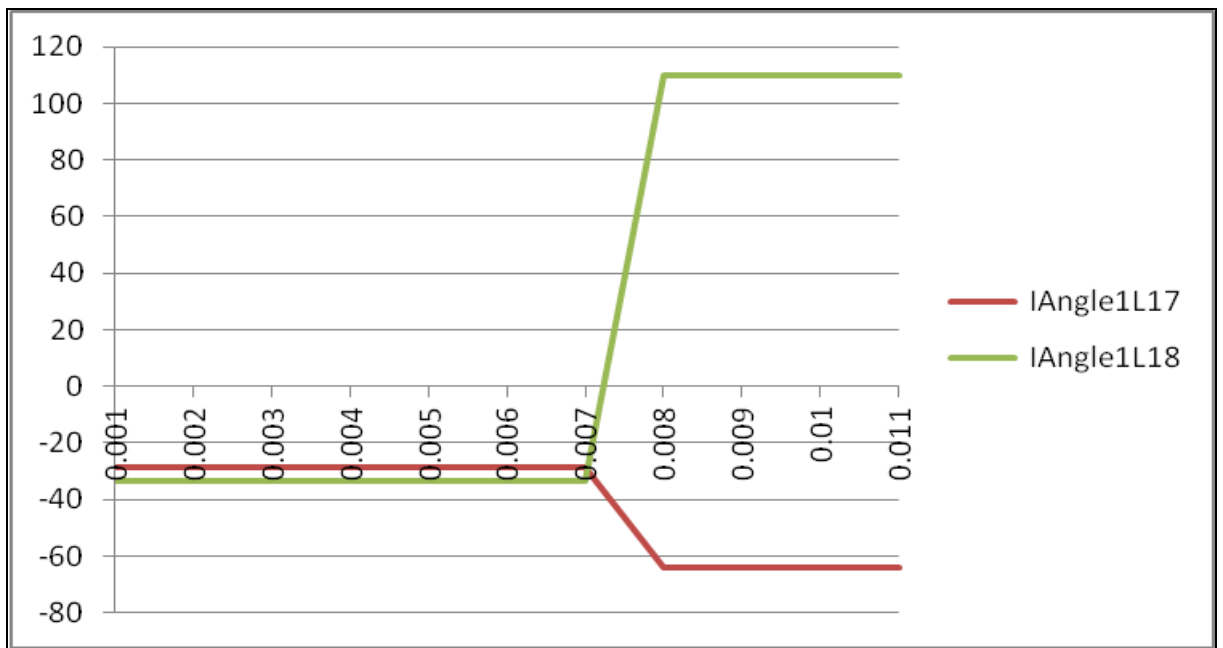

Figure 4-12: I1 phase angle reversal for fault at 730 node

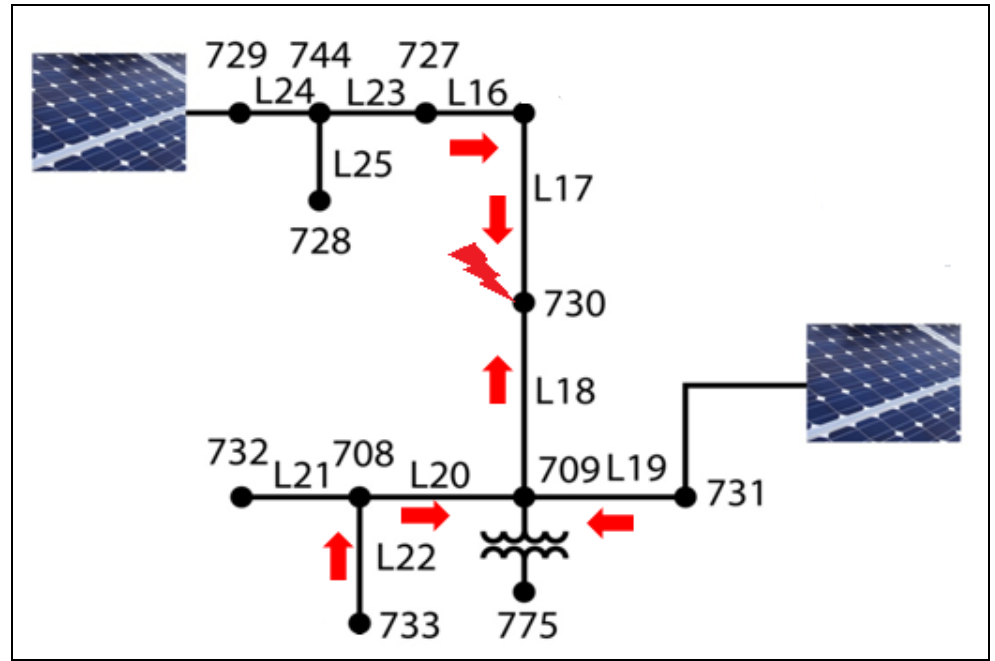

Figure 4-13 Power flow in zone2 during fault at 730 


\subsubsection{Three phase to ground fault at node 708}

In this test case, a $3 \mathrm{LG}$ fault is simulated at 708. Since this is a zone2 fault, SW2 acts will observe a violation of the positive sequence current magnitudes. L17, L18 and L20 violate the limits. For a $3 \mathrm{LG}$ fault in zone2, $\mathrm{I}_{1}$ th is $900 \mathrm{~A}$ (Table 4-23) and the values of $\mathrm{I}_{1}$ in all the lines in the test system for the 3LG fault at node 708 (zone2) can be seen from Table 4-17. The power flow in all the lines in the system after the fault is shown in Table 4-52. The power flow result shows that the real power flows in the same direction in the lines L17, L18 and L20. However, the flow in the line L22 will flow in the opposite direction of the line L20. Hence, the agent will decide that the faulty node is 708 . The power flow in zone 2 after the fault at 730 is depicted in the Figure4-17. Also, the change in the phase angles of the positive sequence current of the lines L20 and L22 will be opposite. The plot of the $\mathrm{I}_{1}$ phase angles for the lines L20 and L22 shown in the Figure414 confirms this. The fault is simulated at 0.007 seconds.

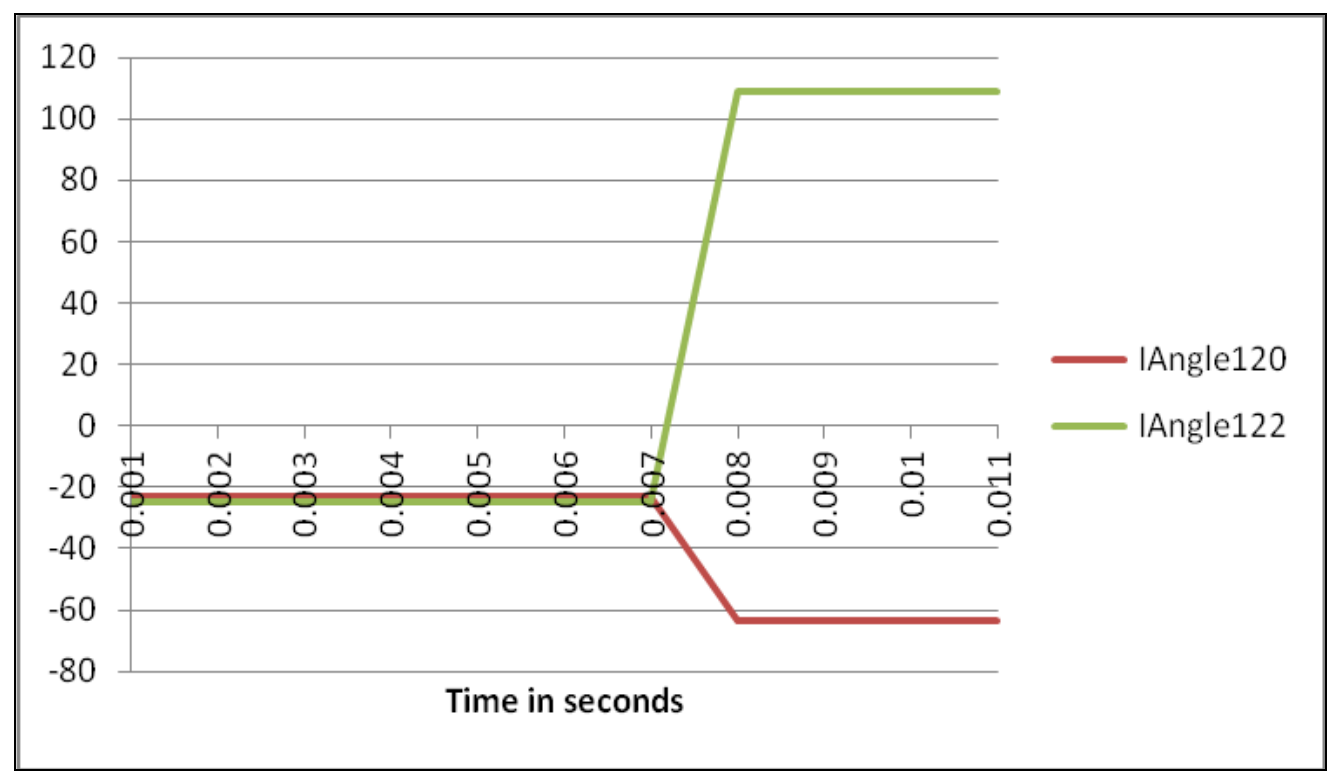

Figure 4-14: $I_{1}$ phase angle reversal for fault at 708 node 
The JADE output for this case is shown in the figure below:

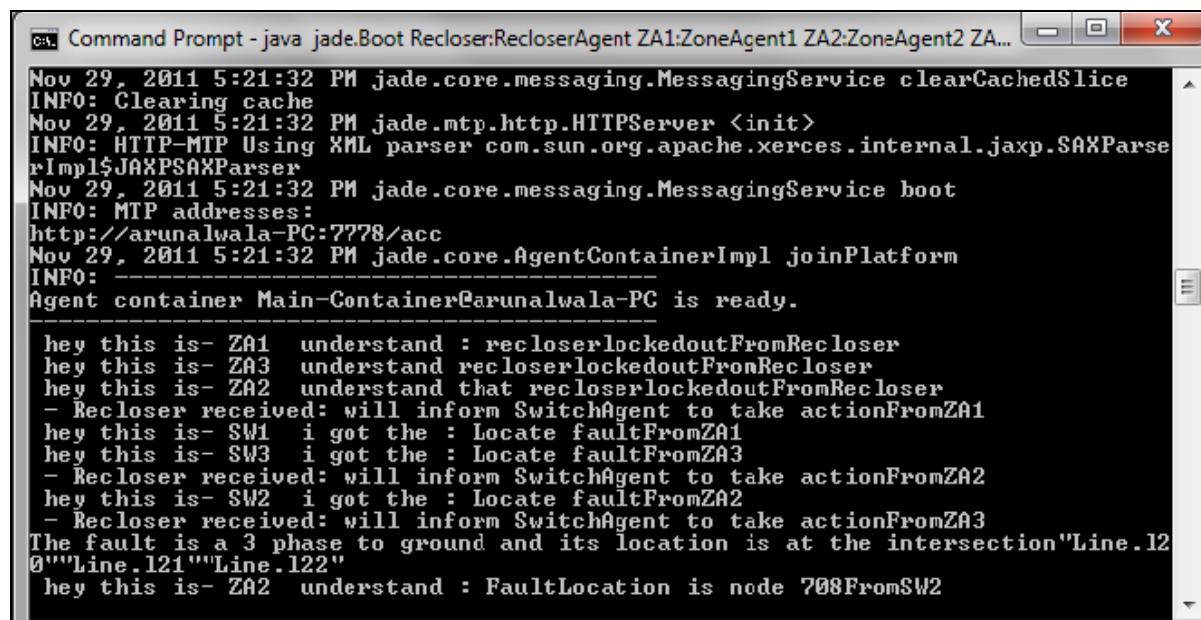

Figure 4-15: JADE Output for fault at 708 Node

The message exchange between the agents from sniffer agent GUI for the fault location in zone2 is represented in Figure 4-16.

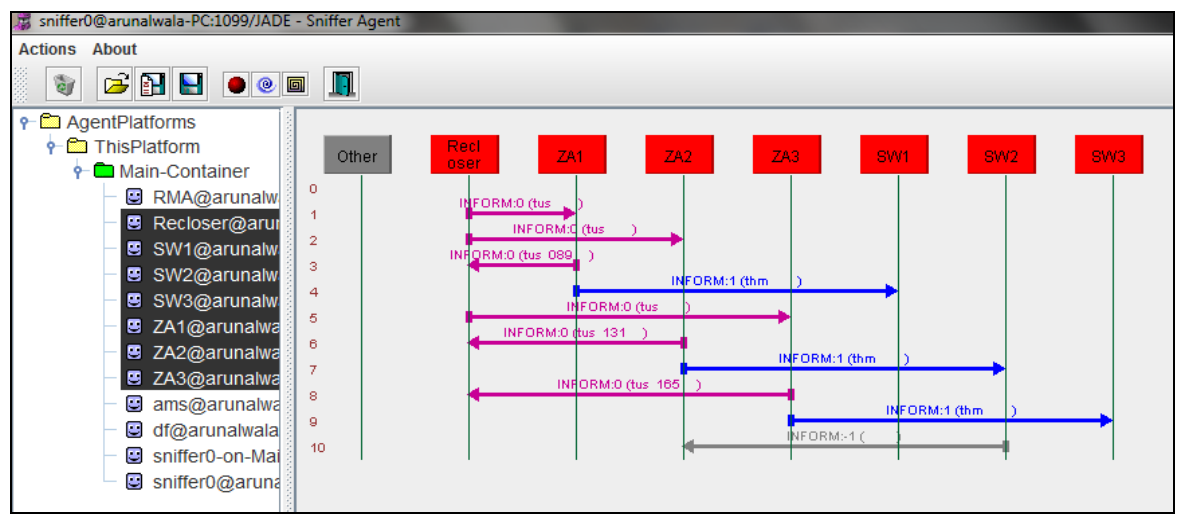

Figure 4-16: Agent Message exchange for Fault Location in zone2 


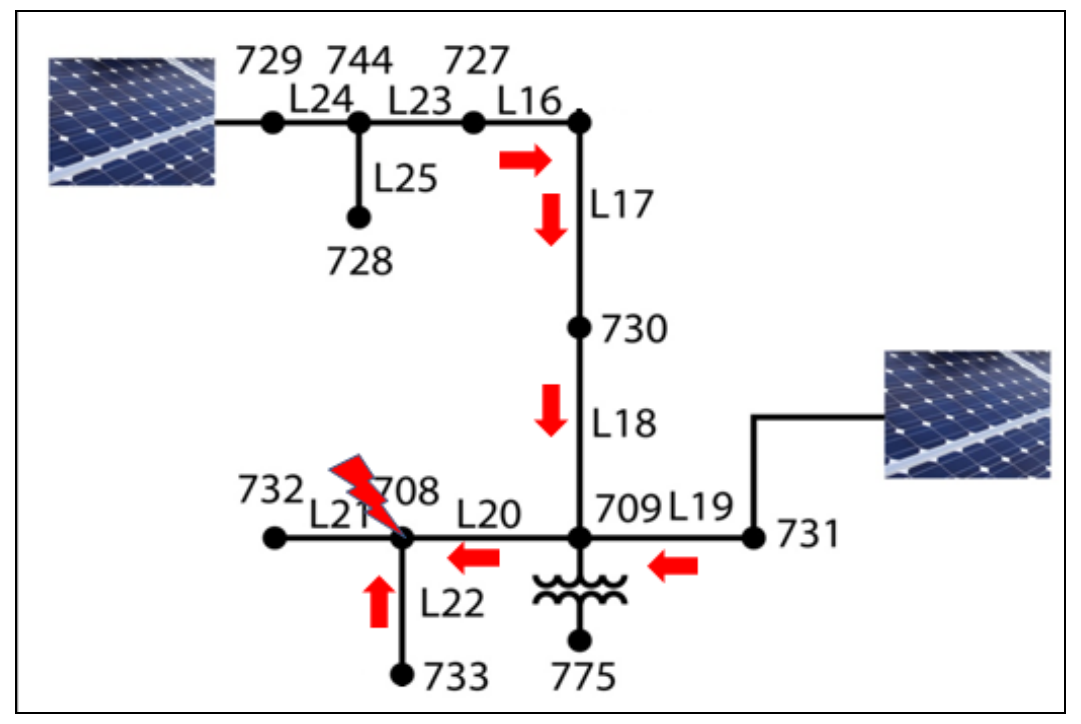

Figure 4-17 Power flow in zone2 during fault at 730

\subsubsection{Line to Line fault at node 738}

In this test case, a LL fault is simulated at 738. The Switch Agents in all the zones start checking sequence current magnitudes and SW3 notices violation in $\mathrm{I}_{2}$ th of L27 and L28. For a LL fault in zone3, $I_{2}$ th is $470 \mathrm{~A}$ (Table 4-23) and the values of $\mathrm{I}_{3}$ in all the lines in the test system for the LL fault at node 738 (zone3) can be seen from Table 4-22. The power flow in all the lines in the system after the fault is shown in Table 4-53.Then, the condition for current reversal for a line to line fault in L27 and L28 are checked. The current reversal for LL fault is determined by checking direction of real power in two phases of the distribution line. When there is a faulty node with generation sources on either side of it, the real power in at least two phases of the adjoining lines of it will be opposite. The power flow results clearly show that the real power will be opposite in phases 1 and 2 for the lines L28 and L29. Hence, the agent locates the fault to be between these lines at 738. The JADE output for this case is shown in the figure below: 


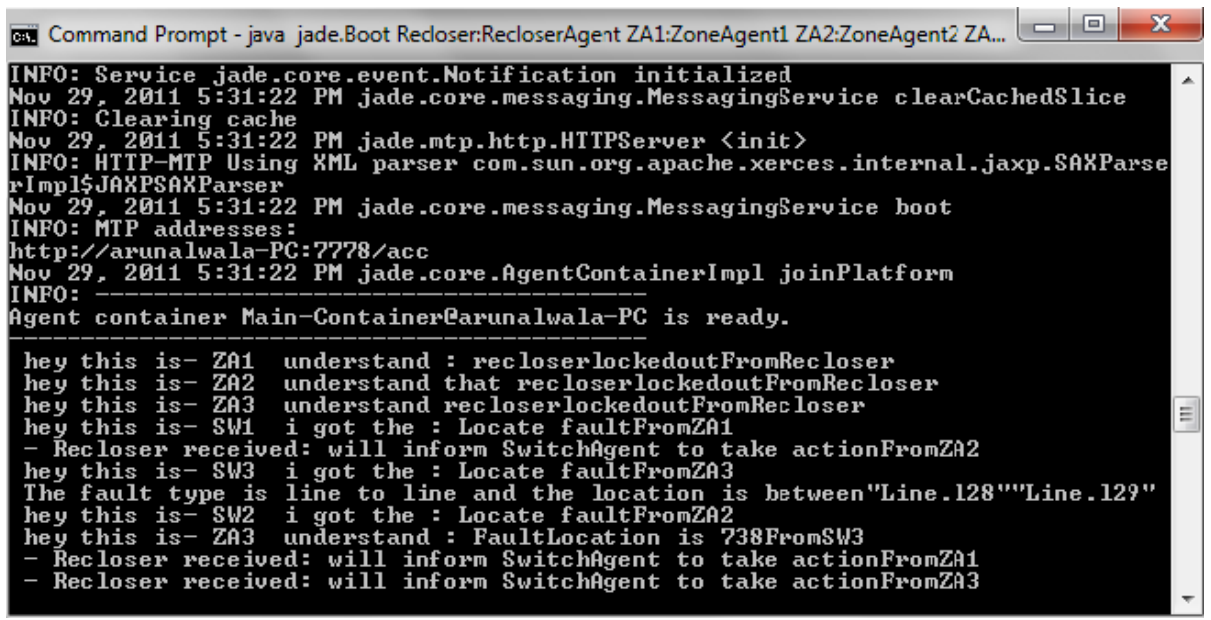

Figure 4-18: JADE Output for fault at 738 Node

The message exchange between the agents from sniffer agent GUI for the fault location in zone3 is represented in Figure 4-19.

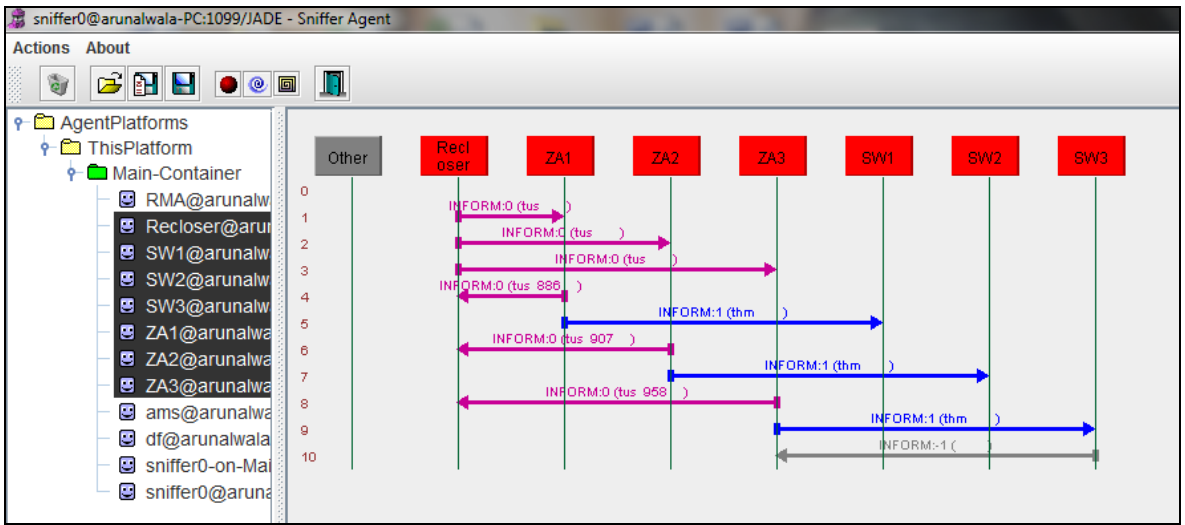

Figure 4-19: Agent Message exchange for Fault Location in Zone3 
Table 4-51 Power flow for 3LG fault at node 730

\begin{tabular}{|c|c|c|c|c|c|c|c|c|c|c|c|c|}
\hline Element & kW1_1 & kvar1_1 & kW2_1 & kvar2_1 & kW3_1 & kvar3_1 & kW1_2 & kvar1 & kW2_2 & kvar2_2 & kW3_2 & kvar3_2 \\
\hline Subxf & 739.23 & 647.62 & 702.39 & 656.20 & 670.60 & 687.08 & 0.00 & 0.00 & -589.08 & -147.40 & -629.83 & -157.52 \\
\hline Xfm1 & 0.00 & 0.00 & 0.00 & 0.00 & 0.00 & 0.00 & 0.00 & 0.00 & 0.00 & 0.00 & 0.00 & 0.00 \\
\hline Line.11 & 530.46 & 91.60 & 533.67 & 99.77 & 508.08 & 117.23 & -497.09 & -62.65 & -505.35 & -70.02 & -479.79 & -82.24 \\
\hline Line.12 & -36.75 & -6.00 & -37.08 & -8.55 & -33.45 & -8.23 & 37.10 & 6.10 & 37.44 & 8.66 & 33.74 & 8.34 \\
\hline Line.13 & -66.23 & -11.92 & -52.60 & -23.97 & -47.01 & -7.21 & 66.78 & 12.22 & 52.99 & 24.17 & 47.26 & 7.38 \\
\hline Line.14 & 600.07 & 80.56 & 595.03 & 102.55 & 560.24 & 97.67 & -533.28 & -22.24 & -540.49 & -45.22 & -507.16 & -31.73 \\
\hline Line.15 & -69.80 & -13.92 & -52.99 & -24.17 & -50.19 & -5.68 & 70.66 & 14.38 & 53.55 & 24.46 & 50.59 & 5.96 \\
\hline Line.16 & -35.01 & -3.87 & -20.53 & -18.66 & -13.86 & 0.77 & 35.32 & 4.03 & 20.72 & 18.76 & 13.91 & -0.74 \\
\hline Line.17 & 0.00 & -0.01 & 1.49 & -0.88 & 1.44 & 0.87 & 0.00 & 0.00 & -1.48 & 0.88 & -1.44 & -0.88 \\
\hline Line.18 & 0.00 & 0.00 & 1.48 & -0.88 & 1.44 & 0.88 & 0.00 & 0.00 & -1.48 & 0.88 & -1.44 & -0.88 \\
\hline Line.19 & -38.46 & -5.79 & -22.21 & -17.87 & -18.35 & 1.63 & 39.28 & 6.04 & 22.65 & 18.00 & 18.54 & -1.55 \\
\hline Line.110 & 2.81 & 1.55 & 18.05 & -10.73 & 20.04 & 9.20 & -2.81 & -1.56 & -18.02 & 10.74 & -20.00 & -9.19 \\
\hline Line. 111 & -42.10 & -7.60 & -40.70 & -7.27 & -38.58 & -7.64 & 42.90 & 7.84 & 41.45 & 7.48 & 39.27 & 7.89 \\
\hline Line. 112 & -35.66 & -10.52 & -33.02 & -5.80 & -36.73 & -6.73 & 35.73 & 10.54 & 33.07 & 5.81 & 36.80 & 6.76 \\
\hline Line.113 & -37.93 & -9.30 & -37.90 & -5.48 & -39.40 & -8.32 & 38.42 & 9.45 & 38.36 & 5.62 & 39.91 & 8.50 \\
\hline Line.114 & 3.03 & 1.71 & 0.00 & 0.00 & 2.94 & -1.71 & -3.03 & -1.71 & 0.00 & 0.00 & -2.94 & 1.71 \\
\hline Line.115 & -40.13 & -7.81 & -37.44 & -8.66 & -36.68 & -6.63 & 40.46 & 7.91 & 37.73 & 8.74 & 36.96 & 6.73 \\
\hline Line.116 & -29.27 & -7.51 & -30.77 & -1.11 & -34.96 & -5.15 & 29.45 & 7.57 & 30.95 & 1.16 & 35.19 & 5.23 \\
\hline Line. 117 & 562.55 & 29.75 & 571.26 & 46.33 & 542.11 & 36.87 & -479.38 & 14.51 & -492.37 & -5.48 & -472.89 & 9.56 \\
\hline Line.118 & -69.39 & -15.41 & -76.66 & 5.48 & -92.11 & -8.66 & 70.01 & 15.74 & 77.30 & -5.15 & 93.01 & 9.24 \\
\hline Line.119 & -34.05 & -2.60 & -32.95 & -3.69 & -33.09 & -1.18 & 34.46 & 2.82 & 33.30 & 3.87 & 33.42 & 1.40 \\
\hline Line.120 & -35.96 & -13.13 & -44.36 & 8.84 & -59.93 & -8.06 & 36.25 & 13.29 & 44.70 & -8.66 & 60.53 & 8.45 \\
\hline Line.121 & 0.86 & 0.51 & 0.00 & 0.00 & 0.88 & -0.51 & -0.86 & -0.51 & 0.00 & 0.00 & -0.88 & 0.51 \\
\hline Line.122 & -37.11 & -13.79 & -44.70 & 8.66 & -61.42 & -7.94 & 37.41 & 13.96 & 45.05 & -8.48 & 62.04 & 8.33 \\
\hline
\end{tabular}




\begin{tabular}{|c|c|c|c|c|c|c|c|c|c|c|c|c|}
\hline Element & kW1_1 & kvar1_1 & kW2_1 & kvar2_1 & kW3_1 & kvar3_1 & kW1_2 & kvar1 & kW2_2 & kvar2_2 & kW3_2 & kvar3_2 \\
\hline Line.123 & -30.60 & -8.24 & -30.95 & -1.16 & -36.33 & -4.56 & 30.72 & 8.30 & 31.05 & 1.21 & 36.48 & 4.66 \\
\hline Line.124 & -34.23 & -7.62 & -34.62 & -1.89 & -38.79 & -4.66 & 34.51 & 7.70 & 34.87 & 1.97 & 39.12 & 4.78 \\
\hline Line.125 & 2.34 & -0.01 & 2.37 & 0.00 & 2.31 & 0.00 & -2.33 & 0.01 & -2.37 & 0.00 & -2.31 & 0.00 \\
\hline Line.126 & -45.59 & -9.10 & -53.39 & 3.62 & -62.04 & -8.33 & 46.30 & 9.47 & 54.23 & -3.19 & 63.12 & 9.02 \\
\hline Line.127 & -13.18 & -8.87 & -18.99 & 6.60 & -30.21 & -5.82 & 13.28 & 8.92 & 19.13 & -6.54 & 30.50 & 6.00 \\
\hline Line.128 & -27.03 & -0.67 & -33.09 & -1.71 & -30.50 & -6.00 & 27.19 & 0.75 & 33.31 & 1.82 & 30.68 & 6.11 \\
\hline Line.129 & -29.94 & 0.90 & -36.10 & -3.47 & -30.68 & -6.11 & 30.13 & -0.80 & 36.36 & 3.60 & 30.85 & 6.23 \\
\hline Line.130 & -32.05 & -0.32 & -36.36 & -3.60 & -32.84 & -5.10 & 32.27 & 0.44 & 36.62 & 3.73 & 33.03 & 5.23 \\
\hline Line.131 & 1.92 & 1.12 & 0.00 & 0.00 & 1.99 & -1.12 & -1.92 & -1.12 & 0.00 & 0.00 & -1.98 & 1.12 \\
\hline Line.132 & -34.02 & -1.14 & -35.23 & -3.41 & -33.85 & -2.67 & 34.65 & 1.33 & 35.87 & 3.60 & 34.41 & 2.87 \\
\hline Line.133 & -36.56 & -2.45 & -35.87 & -3.60 & -36.37 & -1.75 & 38.29 & 2.98 & 37.44 & 4.06 & 37.90 & 2.31 \\
\hline Line.134 & 1.91 & 1.12 & 0.00 & 0.00 & 1.96 & -1.12 & -1.91 & -1.12 & 0.00 & 0.00 & -1.96 & 1.12 \\
\hline
\end{tabular}

Table 4-52 Power flow 3LG fault 708

\begin{tabular}{|c|c|c|c|c|c|c|c|c|c|c|c|c|}
\hline Element & kW1_1 & kvar1_1 & kW2_1 & kvar2_1 & kW3_1 & kvar3_1 & kW1_2 & kvar1 & kW2_2 & kvar2_2 & kW3_2 & kvar3_2 \\
\hline Subxf & 770.88 & 638.18 & 738.78 & 646.25 & 700.62 & 679.17 & 0.00 & 0.00 & -625.73 & -153.61 & -676.47 & -170.07 \\
\hline Xfm1 & 0.00 & 0.00 & 0.00 & 0.00 & 0.00 & 0.00 & 0.00 & 0.00 & 0.00 & 0.00 & 0.00 & 0.00 \\
\hline Line.11 & 571.19 & 106.76 & 577.36 & 112.95 & 543.73 & 134.04 & -539.38 & -79.17 & -550.21 & -84.43 & -516.67 & -100.57 \\
\hline Line.12 & -39.78 & -8.05 & -40.40 & -11.02 & -35.56 & -10.79 & 40.12 & 8.15 & 40.76 & 11.12 & 35.84 & 10.89 \\
\hline Line.13 & -75.67 & -20.46 & -61.27 & -33.51 & -53.83 & -15.57 & 76.27 & 20.78 & 61.72 & 33.75 & 54.11 & 15.77 \\
\hline Line.14 & 654.83 & 107.68 & 651.87 & 128.96 & 606.06 & 126.94 & -590.23 & -51.32 & -598.67 & -73.02 & -554.60 & -62.99 \\
\hline Line. 15 & -80.01 & -22.88 & -61.72 & -33.75 & -57.70 & -13.67 & 80.96 & 23.39 & 62.38 & 34.09 & 58.16 & 13.99 \\
\hline Line.16 & -42.38 & -10.01 & -26.52 & -26.52 & -18.29 & -4.95 & 42.77 & 10.21 & 26.80 & 26.66 & 18.36 & 5.00 \\
\hline Line.17 & 0.00 & -0.01 & 1.84 & -1.09 & 1.77 & 1.08 & 0.00 & 0.00 & -1.84 & 1.09 & -1.77 & -1.08 \\
\hline Line.18 & 0.00 & 0.00 & 1.84 & -1.09 & 1.77 & 1.08 & 0.00 & 0.00 & -1.83 & 1.08 & -1.77 & -1.08 \\
\hline
\end{tabular}




\begin{tabular}{|c|c|c|c|c|c|c|c|c|c|c|c|c|}
\hline Element & kW1_1 & kvar1_1 & kW2_1 & kvar2_1 & kW3_1 & kvar3_1 & kW1_2 & kvar1 & kW2_2 & kvar2_2 & kW3_2 & kvar3_2 \\
\hline Line.19 & -46.66 & -12.38 & -28.64 & -25.57 & -23.81 & -3.90 & 47.68 & 12.69 & 29.29 & 25.76 & 24.07 & 4.00 \\
\hline Line.110 & 3.14 & 1.73 & 20.13 & -11.96 & 22.17 & 10.25 & -3.14 & -1.73 & -20.10 & 11.97 & -22.14 & -10.24 \\
\hline Line.111 & -50.82 & -14.42 & -49.42 & -13.80 & -46.24 & -14.25 & 51.81 & 14.72 & 50.35 & 14.07 & 47.10 & 14.56 \\
\hline Line.112 & -38.58 & -13.38 & -35.86 & -7.56 & -39.87 & -9.04 & 38.65 & 13.40 & 35.91 & 7.58 & 39.93 & 9.06 \\
\hline Line.113 & -41.11 & -12.03 & -41.29 & -7.21 & -42.80 & -10.80 & 41.58 & 12.17 & 41.73 & 7.33 & 43.30 & 10.98 \\
\hline Line.114 & 3.74 & 2.10 & 0.00 & 0.00 & 3.60 & -2.10 & -3.74 & -2.10 & 0.00 & 0.00 & -3.59 & 2.10 \\
\hline Line.115 & -43.86 & -10.25 & -40.76 & -11.12 & -39.44 & -8.79 & 44.19 & 10.35 & 41.04 & 11.20 & 39.70 & 8.89 \\
\hline Line.116 & -35.99 & -14.33 & -38.08 & -6.70 & -42.16 & -11.81 & 36.22 & 14.40 & 38.30 & 6.77 & 42.45 & 11.91 \\
\hline Line.117 & 626.21 & 65.64 & 636.75 & 79.72 & 596.76 & 74.79 & -544.61 & -22.25 & -558.73 & -39.31 & -528.57 & -29.11 \\
\hline Line.118 & 54264 & 2109 & 558.73 & 39.31 & 526.60 & 3027 & -51564 & -674 & -53273 & -2583 & -504.05 & -1516 \\
\hline Line. 120 & 552.12 & 11.62 & 568.06 & 31.60 & 539.07 & 18.37 & -502.58 & 14.73 & -520.71 & -7.07 & -497.76 & 9.31 \\
\hline Line.122 & -37.01 & -15.22 & -44.74 & 7.07 & -60.95 & -8.82 & 37.34 & 15.39 & 45.09 & -6.89 & 61.60 & 9.23 \\
\hline Line.121 & 0.81 & 0.48 & 0 & -0.00 & 0.83 & -0.48 & -0.81 & -0.48 & 0 & 0 & -0.83 & 0.48 \\
\hline Line.119 & -36.48 & -4.87 & -35.33 & -5.76 & -35.02 & -3.21 & 36.89 & 5.09 & 35.68 & 5.94 & 35.35 & 3.43 \\
\hline Line.123 & -37.68 & -15.24 & -38.30 & -6.77 & -43.87 & -11.07 & 37.84 & 15.32 & 38.43 & 6.83 & 44.05 & 11.19 \\
\hline Line.124 & -42.32 & -14.45 & -42.95 & -7.70 & -46.93 & -11.19 & 42.67 & 14.56 & 43.28 & 7.79 & 47.33 & 11.33 \\
\hline Line.125 & 2.98 & -0.01 & 3.01 & 0.01 & 2.88 & 0.01 & -2.98 & 0.01 & -3.01 & -0.01 & -2.88 & -0.01 \\
\hline Line.126 & -45.32 & -10.66 & -53.31 & 2.15 & -61.60 & -9.23 & 46.06 & 11.05 & 54.17 & -1.71 & 62.73 & 9.96 \\
\hline Line.127 & -13.31 & -9.52 & -19.09 & 5.72 & -30.02 & -6.27 & 13.42 & 9.58 & 19.23 & -5.66 & 30.33 & 6.45 \\
\hline Line. 128 & -26.85 & -1.53 & -33.00 & -2.39 & -30.33 & -6.45 & 27.02 & 1.61 & 33.23 & 2.51 & 30.52 & 6.57 \\
\hline Line.129 & -29.66 & $\begin{array}{l}-0.03 \\
\end{array}$ & -35.93 & $\begin{array}{l}-4.09 \\
\end{array}$ & -30.52 & -6.57 & 29.86 & 0.13 & 36.20 & 4.22 & 30.70 & 6.69 \\
\hline Line. 130 & -31.68 & -1.21 & -36.20 & -4.22 & -32.58 & -5.61 & 31.90 & 1.33 & 36.47 & 4.36 & 32.79 & 5.75 \\
\hline Line.131 & 1.82 & 1.08 & 0.00 & 0.00 & 1.89 & -1.08 & -1.82 & -1.08 & 0.00 & 0.00 & -1.88 & 1.08 \\
\hline Line.132 & -33.61 & -2.05 & -35.08 & -4.01 & -33.59 & -3.18 & 34.26 & 2.24 & 35.73 & 4.20 & 34.17 & 3.39 \\
\hline Line.133 & -36.07 & -3.31 & -35.73 & -4.20 & -36.03 & -2.32 & 37.84 & 3.86 & 37.35 & 4.67 & 37.61 & 2.89 \\
\hline Line.134 & 1.81 & 1.07 & 0.00 & 0.00 & 1.86 & -1.07 & -1.81 & -1.07 & 0.00 & 0.00 & -1.86 & 1.07 \\
\hline
\end{tabular}


Table 4-53 Power flow for LL fault at 738

\begin{tabular}{|c|c|c|c|c|c|c|c|c|c|c|c|c|}
\hline Element & kW1_1 & kvar1_1 & kW2_1 & kvar2_1 & kW3_1 & kvar3_1 & kW1_2 & kvar1 & kW2_2 & kvar2_2 & kW3_2 & kvar3_2 \\
\hline Subxf & 1270.85 & 194.87 & -26.27 & 928.27 & 335.40 & 211.13 & 0.00 & 0.00 & -1212.62 & 196.59 & 111.02 & -695.82 \\
\hline Xfm1 & 0.00 & 0.00 & 0.00 & 0.00 & 0.00 & 0.00 & 0.00 & 0.00 & 0.00 & 0.00 & 0.00 & 0.00 \\
\hline Line.11 & 1118.85 & -216.60 & -125.18 & 607.59 & 304.80 & 187.40 & -1096.96 & 238.63 & 141.60 & -590.36 & -303.87 & -185.76 \\
\hline Line.12 & -83.50 & 32.22 & 34.93 & -49.28 & 39.09 & 28.89 & 83.78 & -32.15 & -34.55 & 49.38 & -39.05 & -28.92 \\
\hline Line.13 & -155.58 & 49.41 & 91.55 & -107.06 & 142.87 & 43.90 & 156.00 & -49.19 & -90.61 & 107.54 & -142.68 & -43.85 \\
\hline Line.14 & 1336.04 & -320.27 & -268.08 & 746.69 & 121.91 & 112.97 & -1291.86 & 365.99 & 306.64 & -706.19 & -121.80 & -112.33 \\
\hline Line. 15 & -188.61 & 42.78 & 90.61 & -107.54 & 95.38 & 50.25 & 189.47 & -42.31 & -89.25 & 108.24 & -95.23 & -50.25 \\
\hline Line.16 & -86.09 & 25.46 & 64.70 & -71.74 & 126.32 & 19.05 & 86.36 & -25.35 & -63.70 & 72.24 & -126.03 & -18.98 \\
\hline Line.17 & 0.00 & -0.04 & 6.99 & -3.27 & 18.64 & 3.19 & 0.00 & 0.01 & -6.99 & 3.27 & -18.63 & -3.24 \\
\hline Line. 18 & 0.00 & -0.01 & 6.99 & -3.27 & 18.63 & 3.24 & 0.00 & 0.00 & -6.98 & 3.26 & -18.63 & -3.26 \\
\hline Line.19 & -119.15 & 18.85 & 56.71 & -68.97 & 60.31 & 22.34 & 120.30 & -18.52 & -54.80 & 69.52 & -60.14 & -22.37 \\
\hline Line.110 & 8.01 & 1.60 & 27.90 & -13.56 & 86.67 & 11.97 & -8.01 & -1.61 & -27.87 & 13.57 & -86.63 & -11.96 \\
\hline Line.111 & -128.31 & 16.91 & 26.90 & -55.96 & -26.52 & 10.41 & 129.40 & -16.59 & -26.12 & 56.17 & 26.55 & -10.45 \\
\hline Line.112 & -103.37 & 16.85 & 24.55 & -36.50 & -31.09 & 31.20 & 103.45 & -16.83 & -24.51 & 36.51 & 31.10 & -31.21 \\
\hline Line.113 & -108.77 & 20.64 & 20.30 & -38.36 & -42.47 & 29.25 & 109.33 & -20.47 & -20.05 & 38.43 & 42.53 & -29.26 \\
\hline Line.114 & 32.64 & 6.39 & 0.00 & 0.00 & 47.33 & -6.40 & -32.61 & -6.39 & 0.00 & 0.00 & -47.30 & 6.39 \\
\hline Line.115 & -116.41 & 25.75 & 34.55 & -49.38 & -8.28 & 35.32 & 116.81 & -25.64 & -34.25 & 49.46 & 8.30 & -35.34 \\
\hline Line.116 & -81.71 & 11.01 & 36.50 & -39.70 & 18.83 & 7.81 & 81.86 & -10.97 & -36.31 & 39.75 & -18.83 & -7.83 \\
\hline Line.117 & 1373.57 & -376.99 & -343.14 & 745.89 & 102.97 & 104.52 & -1315.43 & 409.84 & 399.94 & -716.48 & -102.94 & -104.21 \\
\hline Line.118 & 1288.04 & -414.10 & -399.94 & 716.48 & 61.17 & 108.47 & -1269.38 & 424.64 & 418.87 & -706.68 & -61.18 & -108.42 \\
\hline Line.119 & -121.21 & 31.24 & 48.87 & -44.18 & -12.21 & 38.07 & 121.69 & -30.98 & -48.50 & 44.36 & 12.24 & -38.12 \\
\hline Line.120 & 1390.59 & -455.88 & -467.74 & 750.87 & 73.39 & 70.34 & -1354.92 & 476.10 & 502.87 & -732.68 & -73.42 & -70.25 \\
\hline Line. 121 & 14.44 & 1.98 & 0.00 & 0.00 & 22.63 & -2.01 & -14.43 & -1.99 & 0.00 & 0.00 & -22.62 & 1.99 \\
\hline Line.122 & 1340.48 & -478.08 & -502.87 & 732.68 & 50.79 & 72.26 & -1305.47 & 497.91 & 538.00 & -714.50 & -50.84 & -72.21 \\
\hline Line.123 & -97.55 & 8.24 & 36.31 & -39.75 & -4.34 & 10.56 & 97.67 & -8.18 & -36.20 & 39.81 & 4.34 & -10.59 \\
\hline
\end{tabular}




\begin{tabular}{|c|c|c|c|c|c|c|c|c|c|c|c|c|}
\hline Element & kW1_1 & kvar1_1 & kW2_1 & kvar2_1 & kW3_1 & kvar3_1 & kW1_2 & kvar1 & kW2_2 & kvar2_2 & kW3_2 & kvar3_2 \\
\hline Line.124 & -121.20 & 10.95 & 29.99 & -42.55 & -46.34 & 10.57 & 121.58 & -10.83 & -29.78 & 42.60 & 46.37 & -10.58 \\
\hline Line. 125 & 19.61 & -0.02 & 6.62 & -0.01 & 42.00 & 0.01 & -19.61 & 0.01 & -6.62 & 0.01 & -41.98 & -0.02 \\
\hline Line.126 & 1281.68 & -480.83 & -530.21 & 697.41 & 50.84 & 72.21 & -1222.87 & 514.15 & 589.20 & -666.88 & -50.91 & -72.12 \\
\hline Line.127 & 1301.39 & -554.57 & -533.72 & 702.51 & 15.43 & 37.87 & -1225.12 & 597.93 & 722.09 & -662.46 & -15.51 & -37.89 \\
\hline Line. 128 & 1186.81 & -574.22 & -700.17 & 638.75 & 15.51 & 37.89 & -1142.22 & 599.56 & 745.43 & -615.34 & -15.56 & -37.90 \\
\hline Line.129 & -77.42 & 40.68 & 60.06 & -24.91 & 15.56 & 37.90 & 77.62 & -40.58 & -59.85 & 25.01 & -15.54 & -37.94 \\
\hline Line.130 & -103.11 & 39.16 & 59.85 & -25.00 & -28.66 & 39.37 & 103.44 & -38.98 & -59.65 & 25.10 & 28.69 & -39.39 \\
\hline Line.131 & 25.50 & 1.42 & 0.00 & 0.00 & 44.20 & -1.43 & -25.48 & -1.42 & 0.00 & 0.00 & -44.19 & 1.42 \\
\hline Line.132 & -92.08 & 39.02 & -55.48 & -35.63 & 13.22 & 35.65 & 92.67 & -38.84 & -55.90 & 35.79 & -13.18 & -35.68 \\
\hline Line.133 & -120.27 & 35.93 & 54.90 & -35.79 & -31.89 & 38.60 & 122.58 & -35.21 & -53.45 & 36.20 & 32.04 & -38.64 \\
\hline Line.134 & 27.59 & 2.91 & 0.00 & 0.00 & 45.06 & -2.92 & -27.57 & -2.91 & 0.00 & 0.00 & -45.05 & 2.91 \\
\hline
\end{tabular}




\subsection{Fault Zone Location Scenario}

From an implementation point of view, the algorithm discussed in section 4.3 might not be practical because measurements like sequence current components, phase angles and power flow, for every line in the system might not be available. But, with limited measurements it can be really difficult to ascertain the precise location of the fault. Taking both these perspectives into consideration, this section describes an algorithm for locating the fault zone by using measurements only at the switches shown in the Figure 4-6. The IEEE 37 bus system described in section 4.2 with a Distributed Generation penetration of $25 \%$ is used as the test system for this scenario. The agent architecture, the fault location algorithm and test cases for the fault zone location scenario are described in the subsequent sections.

\subsubsection{Agent Architecture}

The proposed Multi Agent system architecture for Fault Zone Location consists of two categories of agents available.

- Global Agent

- Switch Agents.

Recloser agent (RA) is a Global agent which keeps track of the status of the recloser at the substation and informs the Switch Agent when the recloser locks out. These Switch Agents then communicate among themselves to locate the fault zone.

Each of the Switch Agents represents a switch shown in Figure 4-6.The only information that switches have in this scenario is the magnitude of the positive sequence current. The main responsibility of each agent has been described below:

- Recloser Agent (RA): This agent keeps track of the status of the recloser at the substation and informs the SwitchAgent1 when the recloser locks out. After the lock out, the tripping off action of the recloser has been implemented by opening the substation transformer in test system. 
- SwitchAgent (SW): These agents represent the switches separating each of the zones. These agents communicate among themselves in order to come up with the faulty zone. It is the most important entity in this agent architecture since it locates the faulted zone. The fault zone location algorithm is discussed in section 4.5.2

\subsubsection{Fault Location Algorithm}

The basic concept in the fault zone location algorithm is that during a fault, the power generating sources will tend to feed the fault as a result the current flowing through the switches associated with each of the zones (Switch1, 2, 3 for Zone 1, 2, 3 respectively) will be high. Since the majority of the power (75\%) is coming from the substation, a large amount of current flows through the switches in the upper zones for fault occurring in the zones below them.

Since, the only measurement available at the switches is the magnitude of positive sequence current, the thresholds for fault detection at each of the switches have been set in terms of this measurement. In order to give the thresholds for each of the switches of the various zones, a detailed short circuit study has been conducted by simulating different kinds of fault at various locations in the test system. The faults considered are three phase (3LG), line to line (LL) and single phase to ground (SLG) fault. The results of short circuit study for all these fault types have been shown in the section 4.7.

For a fault in a particular zone, the associated Switch Agent will see a violation in positive sequence current threshold but it will only confirm the fault in its zone only after receiving the threshold status from the Switch Agent of the zone below it. The main reason for taking the decision after getting the threshold status from the other switch agent is because the majority power is coming from the substation and if a fault occurs in a lower zone, the threshold at the switches in the upper zone might be violated. So, to accurately isolate the fault zone the information about threshold status of the other Switch is essential. The test cases for fault zone isolation have been presented in section 4.8 


\subsection{Short Circuit Study}

In this section, the results of the short circuit analysis for all the fault types considered in this work (LL, SLG and 3LG) have been tabulated. The values of I1 for 3LG, SLG LL fault at the switches separating each of the zones in the test system have been tabulated. Based on these values I1th for each of switches have been determined. The resistance of the fault is $0.5 \mathrm{ohm}$.

Table 4-54: I1 value for $3 \mathrm{LG}$ fault

\begin{tabular}{|c|c|c|c|c|}
\hline $\begin{array}{c}\text { Zone } \\
\text { Number }\end{array}$ & $\begin{array}{c}\text { Faulty } \\
\text { Node }\end{array}$ & $\mathbf{S W 1}$ & $\mathbf{S W 2}$ & $\mathbf{S W 3}$ \\
\hline Zone1 & 702 & 728.08 & 80.82 & 27.84 \\
\hline Zone1 & 705 & 714.60 & 76.14 & 25.96 \\
\hline Zone1 & 713 & 719.55 & 78.48 & 27.04 \\
\hline Zone1 & 704 & 707.61 & 75.31 & 26.05 \\
\hline Zone1 & 714 & 705.02 & 74.43 & 25.71 \\
\hline Zone1 & 720 & 689.54 & 70.62 & 24.69 \\
\hline Zone1 & 706 & 676.68 & 67.31 & 23.79 \\
\hline Zone1 & 707 & 661.23 & 61.22 & 21.23 \\
\hline Zone1 & 722 & 657.56 & 60.02 & 20.81 \\
\hline Zone1 & 724 & 640.20 & 54.44 & 18.93 \\
\hline Zone1 & 725 & 668.14 & 64.41 & 22.70 \\
\hline Zone1 & 712 & 706.38 & 73.34 & 24.85 \\
\hline Zone2 & 744 & 697.02 & 683.42 & 26.75 \\
\hline Zone2 & 727 & 703.20 & 690.46 & 27.28 \\
\hline Zone2 & 709 & 693.60 & 679.93 & 28.96 \\
\hline Zone2 & 708 & 686.59 & 671.97 & 29.18 \\
\hline Zone3 & 734 & 668.28 & 651.20 & 698.05 \\
\hline Zone3 & 737 & 653.44 & 633.65 & 677.46 \\
\hline Zone3 & 710 & 655.24 & 636.39 & 680.98 \\
\hline Zone3 & 738 & 647.63 & 627.73 & 670.99 \\
\hline Zone3 & 711 & 640.25 & 619.35 & 661.32 \\
\hline & & & & \\
\hline
\end{tabular}


Table 4-55: I1 value for SLG fault

\begin{tabular}{|c|c|c|c|c|}
\hline $\begin{array}{c}\text { Zone } \\
\text { Number }\end{array}$ & $\begin{array}{c}\text { Faulty } \\
\text { Node }\end{array}$ & SW1 & SW2 & SW3 \\
\hline Zone1 & 702 & 251.524 & 78.369 & 49.493 \\
\hline Zone1 & 705 & 252.14 & 77.7276 & 49.0665 \\
\hline Zone1 & 713 & 251.381 & 78.1568 & 49.2897 \\
\hline Zone1 & 704 & 251.211 & 77.8656 & 49.1436 \\
\hline Zone1 & 714 & 251.312 & 77.7496 & 49.0848 \\
\hline Zone1 & 720 & 251.04 & 77.3548 & 48.8961 \\
\hline Zone1 & 706 & 250.936 & 76.9309 & 48.7008 \\
\hline Zone1 & 707 & 251.747 & 76.3847 & 48.4139 \\
\hline Zone1 & 722 & 251.803 & 76.2655 & 48.3589 \\
\hline Zone1 & 724 & 251.856 & 75.8491 & 48.1607 \\
\hline Zone1 & 725 & 251.169 & 76.6225 & 48.5373 \\
\hline Zone1 & 712 & 252.462 & 77.3314 & 48.866 \\
\hline Zone2 & 744 & 249.752 & 194.632 & 49.174 \\
\hline Zone2 & 727 & 249.819 & 195.254 & 49.2806 \\
\hline Zone2 & 730 & 248.68 & 194.159 & 49.5792 \\
\hline Zone2 & 709 & 248.413 & 193.638 & 49.6061 \\
\hline Zone2 & 708 & 248.007 & 192.655 & 49.6225 \\
\hline Zone3 & 734 & 247.006 & 190.344 & 181.688 \\
\hline Zone3 & 737 & 246.446 & 188.588 & 179.01 \\
\hline Zone3 & 710 & 247.525 & 189.395 & 179.605 \\
\hline Zone3 & 738 & 246.197 & 187.667 & 177.498 \\
\hline Zone3 & 711 & 246.012 & 186.891 & 176.114 \\
\hline
\end{tabular}

Table 4-56: I1 value for $L L$ fault

\begin{tabular}{|c|c|c|c|c|}
\hline $\begin{array}{c}\text { Zone } \\
\text { Number }\end{array}$ & $\begin{array}{c}\text { Faulty } \\
\text { Node }\end{array}$ & SW1 & SW2 & SW3 \\
\hline Zone1 & 702 & 449.777 & 33.0217 & 19.58 \\
\hline Zone1 & 705 & 444.831 & 35.0288 & 21.8093 \\
\hline Zone1 & 713 & 446.288 & 35.3765 & 21.4536 \\
\hline Zone1 & 704 & 441.329 & 37.7279 & 23.4264 \\
\hline Zone1 & 714 & 440.346 & 37.8606 & 23.679 \\
\hline Zone1 & 720 & 433.716 & 40.0773 & 25.5893 \\
\hline Zone1 & 706 & 428.059 & 41.293 & 26.8234 \\
\hline Zone1 & 707 & 422.199 & 40.8372 & 27.4348 \\
\hline Zone1 & 722 & 421.006 & 40.9574 & 27.6649 \\
\hline
\end{tabular}




\begin{tabular}{|c|c|c|c|c|}
\hline $\begin{array}{c}\text { Zone } \\
\text { Number }\end{array}$ & $\begin{array}{c}\text { Faulty } \\
\text { Node }\end{array}$ & SW1 & SW2 & SW3 \\
\hline Zone1 & 724 & 413.616 & 41.7424 & 28.8132 \\
\hline Zone1 & 725 & 424.689 & 41.334 & 27.2941 \\
\hline Zone1 & 712 & 441.743 & 35.7172 & 22.7897 \\
\hline Zone2 & 744 & 435.748 & 403.789 & 22.1544 \\
\hline Zone2 & 727 & 438.417 & 407.346 & 20.9148 \\
\hline Zone2 & 730 & 435.553 & 404.149 & 19.4697 \\
\hline Zone2 & 709 & 433.657 & 401.683 & 19.5203 \\
\hline Zone2 & 708 & 430.536 & 397.548 & 19.6897 \\
\hline Zone3 & 734 & 422.392 & 386.79 & 395.115 \\
\hline Zone3 & 737 & 416.516 & 378.985 & 386.029 \\
\hline Zone3 & 710 & 416.565 & 378.156 & 384.689 \\
\hline Zone3 & 738 & 413.196 & 374.618 & 413.196 \\
\hline Zone3 & 711 & 409.953 & 370.298 & 375.852 \\
\hline
\end{tabular}

Table 4-57: I1th value

\begin{tabular}{|c|c|}
\hline Switch \# & I1th \\
\hline SW1 & 240 \\
\hline SW2 & 180 \\
\hline SW3 & 175 \\
\hline
\end{tabular}

\subsection{Fault Zone Location Test cases}

In this section, the test cases for the fault location in the zones have been described. The simulation is started by simulating the fault on the test system in OpenDss and the values of positive sequence current magnitude are written to an excel file. The agents read the appropriate value to take decisions. The test cases for this scenario are presented in sections 4.8.1, 4.8.2 and 4.8.3.

\subsubsection{Zone1 Fault}

Consider a LL fault simulated at 705 (zone1) in OpenDss. The values of positive sequence current at the switches at the instant before opening substation transformer are written to an excel file. Table 4-56 provides $\mathrm{I}_{1}$ values at the various switches for a $\mathrm{LL}$ fault and $\mathrm{I}_{1}$ th values are available in Table4-57. First, the Recloser agent informs of its locking out action to Switch Agent1 (SW1). Since the LL fault is in Zone1, the 
SwitchAgent1 (SW1) will observe a violation in the value of $\mathrm{I}_{1}$ th (240A) and on observing this, it asks Switch Agent2 (SW2) if its value of $I_{1}$ th (180A) has been violated or not. As seen from the Table 4-56, SW2 will not see a violation and informs the SW1 the same, upon which SW1 will confirm that the faulty zone is zone1. The JADE output for this case is shown in the Figure 4-20 and the message exchange between the agents detected by the sniffer agent is shown in Figure 4-21.

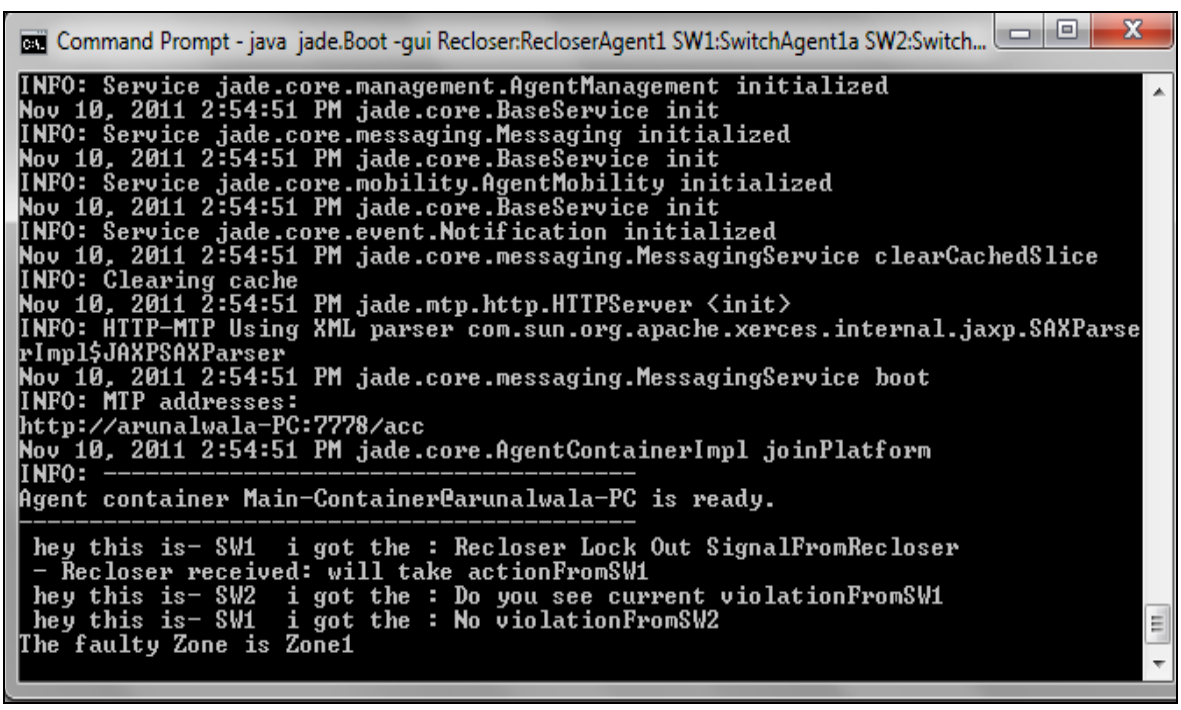

Figure 4-20: JADE Output for fault in Zone1

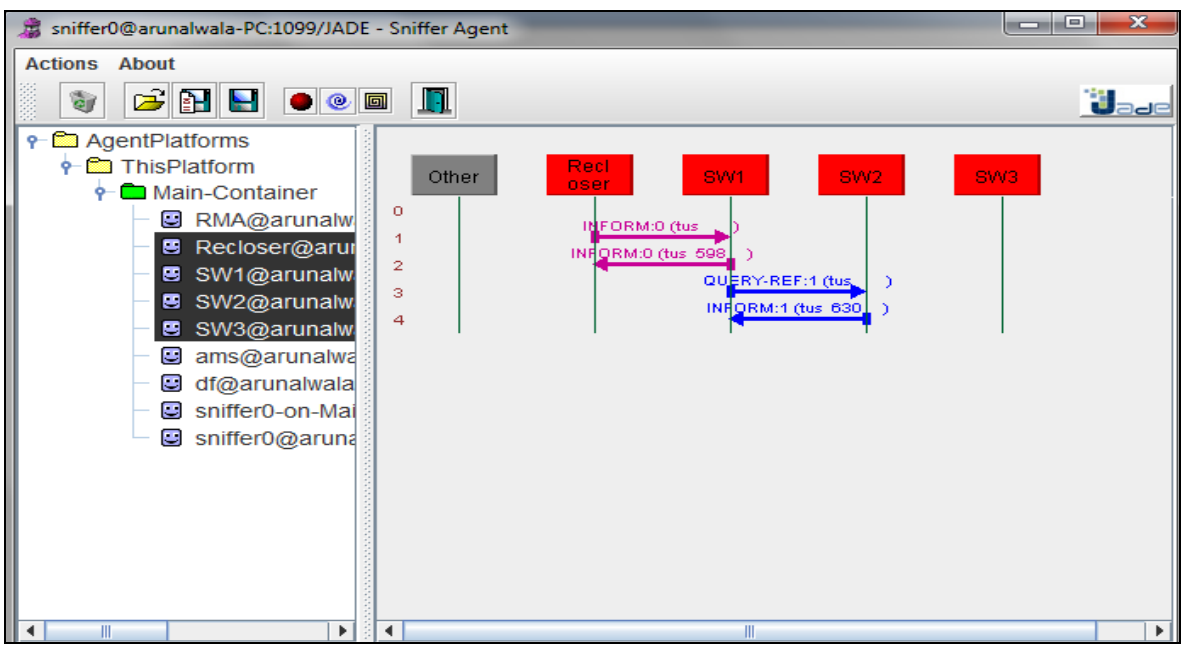

Figure 4-21: Agent Message exchange for Zone1 Fault 


\subsubsection{Zone2 Fault}

Consider that a 3LG fault simulated at node 709 (Zone2) in OpenDss. Table 4-54 provides $I_{1}$ values at the various switches for a $3 L G$ fault and $I_{1}$ th values are available in Table4-57. First, the Recloser agent informs of its locking out action to Switch Agent1 (SW1). SwitchAgent1 (SW1) will observe a violation in the value of $\mathrm{I}_{1}$ th $(240 \mathrm{~A})$ and on observing this it asks Switch Agent2 (SW2), if value of its $I_{1}$ th (180A) has been violated or not. As seen from the Table 4-54, SW2 will see a violation and informs the SW1 about the same, upon which SW1 will not take any action and SW2 will communicate with SwitchAgent3 (SW3) and enquire about its threshold status. SW3 informs SW2 that it doesn't see a violation in its $\mathrm{I}_{1}$ th $(175 \mathrm{~A})$, upon which $\mathrm{SW} 2$ will decide the Zone 2 is the faulty zone. The JADE output for this case is shown in the Figure 4-22 and the message exchange between the agents detected by the sniffer agent is shown in Figure 4-23.

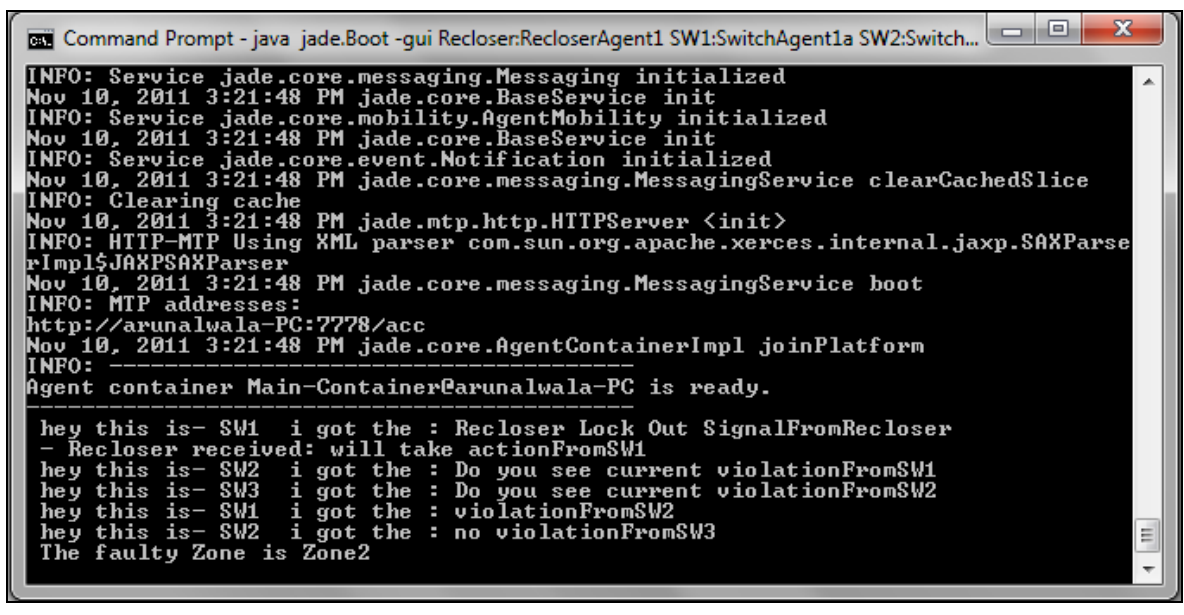

Figure 4-22: JADE Output for fault for Zone2 Fault 


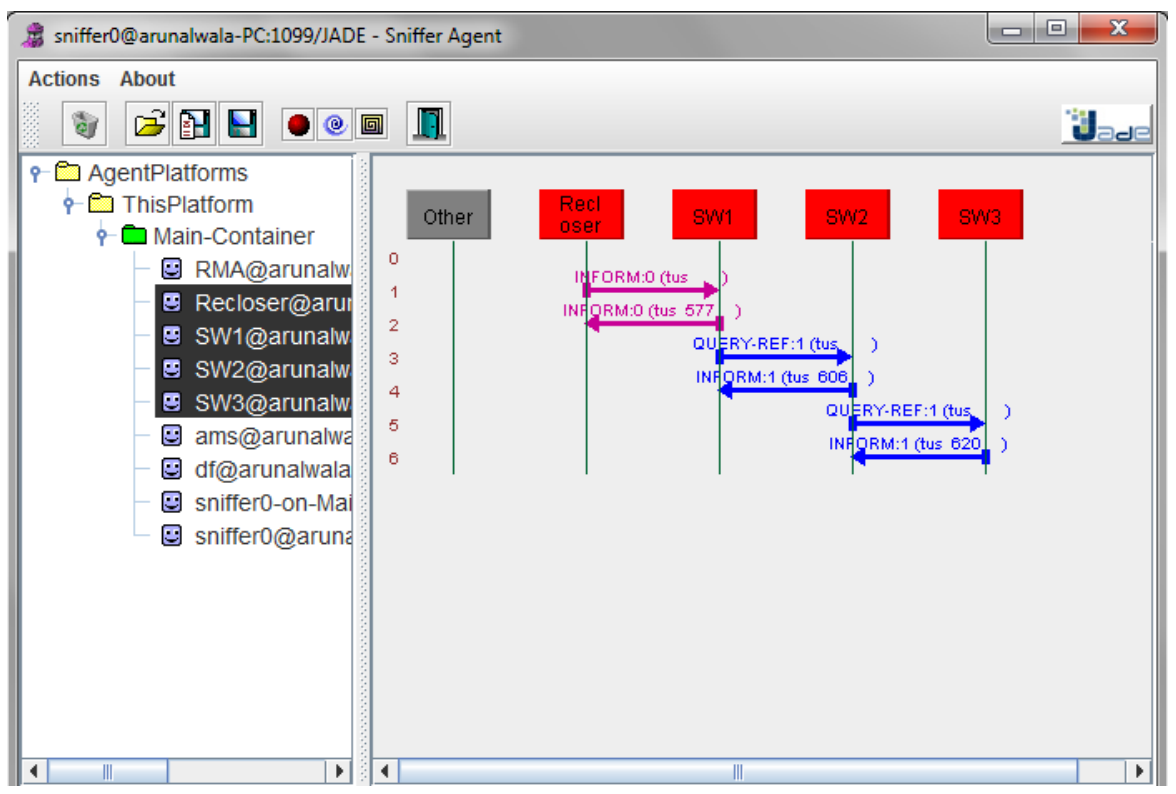

Figure 4-23: Agent Message exchange for Zone2\&Zone3 Fault

\subsubsection{Zone3 Fault}

Consider that a SLG fault simulated at node 734 (Zone3) in OpenDss. Table 4-55 provides $I_{1}$ values at the various switches for a SLG fault and $I_{1}$ th values are available in Table4-57. First, the Recloser agent informs of its locking out action to Switch Agent1 (SW1). SwitchAgent1 (SW1) will observe a violation in the value of $I_{1}$ th (240A) and on observing this it asks Switch Agent2 (SW2), if value of its $I_{1}$ th (180A) has been violated or not. As seen from the Table 4-55, SW2 will see a violation and informs the SW1 about the same, upon which SW1 will not take any action and SW2 will communicate with SwitchAgent3 (SW3) and enquire about its threshold status. SW3 informs SW2 that it has a violation in its $I_{1}$ th $(175 \mathrm{~A})$, upon which SW2 will not take any action. SW3 after receiving the message from SW2 and knowing of its violation will decide that Zone 3 is faulted. The message exchange between the agents detected by the sniffer agent is shown in Figure 4-23 and the JADE output for this case is shown in the Figure 4-24. 


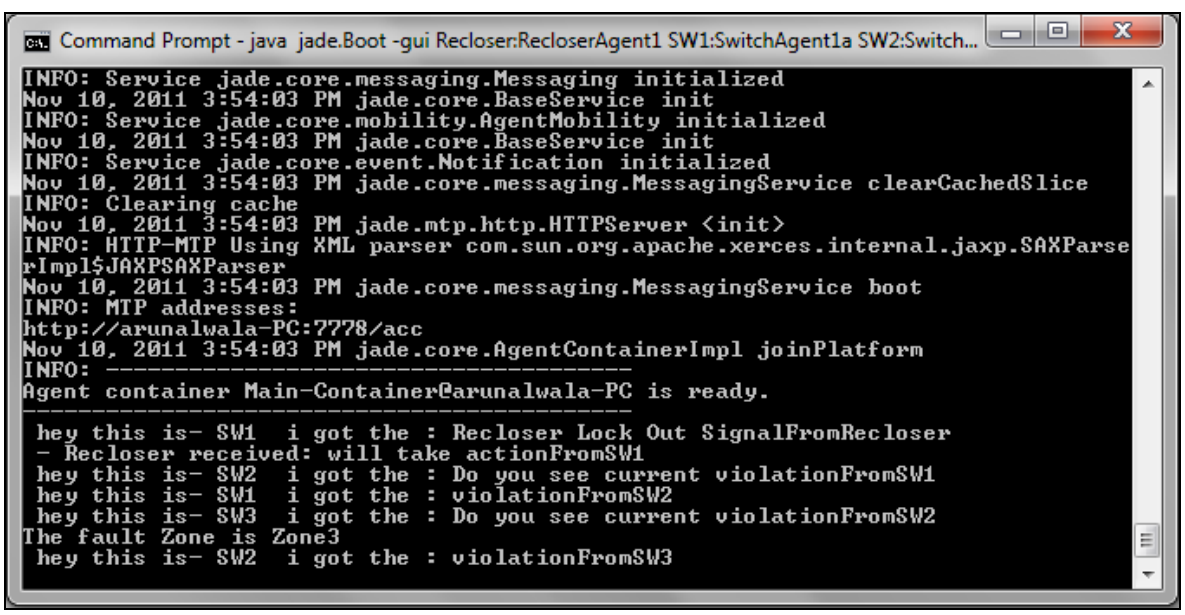

Figure 4-24: JADE Output for Zone3 Fault 


\section{CHAPTER 5}

\section{CONCLUSION AND FUTURE WORK}

The present electric grid is in the transition stage to evolve into a smart grid. One of the leading technologies capable of inducing intelligence into electric grid is the agent technology. Multi agent systems are autonomous, intelligent, distributed and provide faster solutions which are in line with many of the smart grid objectives. Their wide applications in field of power system engineering are ample proof of their capability and their real time deployment on the field would yield really promising results.

\subsection{Conclusion}

In this thesis a Multi agent system model has been implemented in order to determine the fault location in MicroGrid system. The proposed Multi-agent system makes use of a Hierarchical model in which fault detection is done at the top level while the fault location is done at the zonal level. The Microgrid system is developed by adding solar PV model Distributed Generation at various nodes of a benchmark IEEE37 bus system . The proposed model of the Multi Agent system has been implemented on this modified IEEE 37 bus benchmark distribution network. Different fault types like Single line to ground, Line to Line and Three Phase to ground faults have been successfully located by this agent system.

The Multi agent system architecture for fault location for different kinds of faults has been designed and developed using JADE software. All the possible fault cases for different Distributed Generation penetration level in the Microgrid were considered and the results are promising.

\subsection{Future Work}

The MicroGrid is being looked at as one of the alternatives to increase the robustness and reliability of electric power system. Some of the future extensions to the work cited in this thesis are listed below: 
- The next stage of the work shall be focused on the development of an agent system to achieve optimal energy management of the island MicroGrid that will be formed because of the fault isolation at some locations on the IEEE37 bus system considered.

- The work uses a lot of information measurements like sequence current magnitudes and phase angles in all the lines in the system. These kinds of measurements might not be always available and even if available might not be synchronized. So, there is need for work that just takes limited measurements and implements fault location.

- In this work for the implementation of MicroGrid simple steady state model of solar PV generation available in OpenDss software have been used. In order to obtain better results a more dynamic model of the PV model that can be used in the Microgrid can be developed.

- In this work only low impedance faults have been considered but not high impedance faults. So, future work has to focus on the fault location for a high impedance fault.

- This work does not take in to account the scenario where there is a communication failure during message exchange between the agents.

- Future work shall also focus on determining the location of the zonal agent in each of the zones of the test system. 


\section{REFERENCES}

[1] P. Silpa, "Power Market analysis tool for congestion management", M.S Thesis, West Virginia University, 2007

[2] C. Sridhar, "Agent based modeling of Power Distribution system", M.S Thesis, West Virginia University, 2008

[3] http://en.wikipedia.org/wiki/2003_North_America_blackout

[4] “Smart Grid: An Introduction”, Program Concept paper, U.S. Department of Energy, 2001.

[5] M. Wooldridge, N. R. Jennings, "Intelligent agents: Theory and Practice," The Knowledge Engineering Review, Vol. 10, Issue 2, pp 115 - 152, 1995

[6] G. Weiss, "Multi-agent Systems: A Modern Approach to Distributed Artificial Intelligence,’The MIT Press, 2000.

[7] S. Franklin, A.Graesser, "Is it an agent, or just a program?: a taxonomy for autonomous agents", in Proc. the 3rd International Workshop on Agent Theories, Architectures and Languages Springer, Verlag, pp. 21-35, 1996.

[8] H.Nwana, "Software agents: an overview", The Knowledge Engineering Review, 11, No 3,pp. 205-244, 1996

[9] M.J. Bradshaw, 'Software Agents’, Cambridge, MA, MIT Press, 1997.

[10] S. Russell and P.Norvig. Artificial Intelligence: A modern approach. Prentice-Hall, 1995.

[11] http://en.wikipedia.org/wiki/Foundation_for_Intelligent_Physical_Agents

[12] The Foundation for Physical Intelligent Agents, http://www.fipa.org

[13] G. Weiss, " Multiagent Systems: A Modern Approach to Distributed Artificial Intelligence,"The MIT Press, 2000

[14] H.T. Feroze, “MultiAgent Sytems in Microgrids: Design and Implementation”, Master's Thesis, Virginia Polytechnic Institute and State University, 2009.

[15] E. Sortomme, S. S. Venkata, and J. Mitra, "Microgrid protection using communication Assisted digital relays,” IEEE Trans. Power Del., vol.25, no. 4, pp. 2789-2796, 2010.

[16] http://www.transverter.com/micro.html

[17] D. A. Cartes and S. K. Srivastava, "Agent Applications and their future in Power Industry," IEEE Power Engineering Society General Meeting, pp 1 - 6, June 2007.

[18] L. Liu, K. P. Logan, D. A. Cartes and S. K. Srivastava, "Fault Detection, Diagnostics and 
Diagnostics and Prognostics: Software Agent Solutions," IEEE Transactions on Vehicular Technology, Vol. 56, No 4, pp 1613 - 1622, July 2007

[19] R. Isermann, "Process fault detection based on modeling and estimation methods - A survey,” Automatica, Vol. 20, No. 4, pp 387 - 404, July 1984

[20] R. Isermann, "Model based fault detection and diagnosis - Status and Applications," Annual Rev. Control, Vol. 29, pp 71 - 85, 2005

[21] E. Chow and A. Willsky, "Analytical Redundancy and the design of robust failure detection systems," IEEE Transactions on Automatic Control, Vol. 29, No. 7, pp 603 614, July 1984

[22] R. J. Patton and J. Chen, "Advances in Fault Diagnosis using Analytical Redundancy," IEE Proceedings of Integrated Operations Management Control, pp 6/1 - 6/12, 1993

[23] K. Huang, S. K. Srivastava, D. A. Cartes and L. Liu, "Agent Solutions for Navy Shipboard Power Systems," IEEE International Conference on Systems Engineering, pp $1-6$, April 2007

[24] Q. L. Pang, H. L. Gao, and M. J. Xiang, "Multi-agent based fault location algorithm for smart distribution grid," Proceedings of 10th IET International Conference on Developments in Power System Protection. DPSP 2010. The Hilton Deansgate, Manchester, UK, vol.2010, no. 558CP, pp.55, Apr. 2010.

[25] Z. Xiangjun, K.K. Li, W.L. Chan and S. Sheng, "Multi-agents Based Protection for Distributed Generation Systems", Electric Utility Deregulation, Restructuring and Power Technologies. Proc. IEEE International Conference, vol.1, pp. 393-397, Apr. 2004

[26] T. Kato, H. Kanamori, Y. Suzuoki, and T. Funabashi, "Multi-agent based control and protection of power distributed system - protection scheme with simplified information utilization," Proceedings of the 13th International Conference on Intelligent Systems Application to Power Systems, 2005, pages 49-54, Nov. 2005

[27] A.M. El-Zonkoly, "Fault diagnosis in distribution networks with distributed generation", Elsevier Journal, 2011

[28] N. Perera, A.D Rajapakse, A.M. Gole, “ Wavelet-based relay agent for isolating faulty sections in distribution grids with distributed generators", The 8th IEE International Conference on AC and DC Power Transmission, 28-31 March 2006, Pages:162-166

[29] Kueck J. D., Staunton R. H., Labinov S. D. and Kirby B. J. (2003), Microgrid Energy Management System, CERTS, ORNL/TM-2002/242, Jan 2003.

[30] L.Phillips, M.Link, R.Smith, and L.Weiland , "Agent-based control of distributed infrastructure resources”. Sandia National Laboratories, SAND2005-7937, Jan 2006.

[31] A. Dimeas , N.D Hatziargyriou, "Operation of a Multiagent system for microgrid control", IEEE transaction on Power Systems, Vol. 20, No. 3, pp. 1447-1455, 2005. 
[32] A. Dimeas, N.D Hatziargyriou, "Operation of a Multiagent system for microgrid control", in Power Engineering Society General Meeting, Vol. 1, pp. 55-58, 2004.

[33] T. Nagata, Y. Tao, H. Sasaki, H. Fujita, “A Multi-agent Approach to Distribution System Restoration”, in Power Engineering Society General Meeting, Vol. 2, page(s) 660, 2003.

[34] J. Oyarzabal, J. Jimeno, J. Ruela, A. Engler, C. Hardt, “Agent-based-micro-grid management-system", in International Conference onFuture Power Systems, page(s) 6, 2005.

[35] Q. Zhifeng, D. Geert, G. Ning, B. Ronnie, “A Multi-Agent System Architecture for Electrical Energy Matching in a Microgrid"

[36] S. Chouhan, H.J. Hui Wan, A.Feliachi, M.A Choudhry, "Intelligent reconfiguration of smart distribution network using multi-agent technology," Power \& Energy Society General Meeting, 2009. PES '09. IEEE, pp.1-6, 26-30 July 2009.

[37] K. Nareshkumar, M.A. Choudhry, J.Lai, A. Feliachi, "Application of multi-agents for fault detection and reconfiguration of power distribution systems," Power \& Energy Society General Meeting, 2009. PES '09. IEEE

[38] Rabie Belkacemi and Ali Feliachi, "Multi-agent design for power distribution system reconfiguration based on the artificial immune system algorithm", in Proceedings of the 2010 symposium on circuits and systems, pp. 3461-3464, 2010

[39] J. M. Solanki, N. N. Schulz and W. Gao, "Reconfiguration for Restoration of Power Systems using Multi-Agent System," Proceedings of the 37th Annual North American Power Symposium, pp 390 - 395, October 2005.

[40] T. Nagata, Y. Tahara and H. Fujita, "An Agent Approach to Bulk Power System Restoration," IEEE Power Engineering Society General Meeting, Vol. 1, pp 599 - 604, June 2005

[41] T. Nagata, Y. Tao, Y. Tahara and T. Aoyama, "Development of Bulk Power System Restoration Simulator by means of Multi-agent Approach," The $47^{\text {th }}$ IEEE International Midwest Symposium on Circuits and Systems, Vol. 2, pp 337 - 340, July 2004

[42] D. Liu, Y. Chen, G. Shen and Y. Fan, "Multi-agent based Approach for Modeling and Simulation of Bulk Power System Restoration," IEEE/PES Transmission and Distribution Conference and Exhibition, pp 1- 6, 2005

[43] H. F. Wang, "Multi-agent Co-ordination for the Secondary Voltage Control in Power System Contingencies," IEE Proceedings on Generation, Transmission and Distribution, Vol. 148, Issue 1, pp $61-66$, January 2001

[44] S. Gehao, J. Xiuceng and Z. Yi, "Optimal Coordination for Multi-agent based Secondary Voltage Control In Power System," IEEE/PES Transmission and Distribution Conference and Exhibition, pp 1-6, 2005 
[45] J. A. Hossack, J. Menal, S. D. J. McArthur and J. R. McDonald, “A Multi Agent Architecture for Protection Engineering Diagnostic Assistance," IEEE Transactions on Power Systems, Vol. 18, No. 2, pp 639 - 647, May 2003

[46] J. Zhang and Q. Liu, "Application of a Multi-Agent Technology in Relaying Protection," International Conference on Power System Technology, pp 1 - 6, October 2006

[47] D. V. Coury, J. S. Thorp, K. M. Hopkinson and K. P. Birman, "An Agent based Current Differential Relay for use with a Utility Intranet," IEEE Transactions on Power Delivery, Vol. 17, No. 1, pp 47 - 53, January 2002

[48] S. J. Park and J. T. Lim, "Modeling and Control of Agent-based power protection systems using supervisors," IEE Proceedings on Control Theory and Applications, Vol. 153, Issue 1, pp 92 - 98, January 2006

[49] Z. Xiangjun, K. K. Li, W. L. Chan and S. Sheng, "Multi-Agents based Protection for Distributed Generation Systems," Proceedings of the IEEE International Conference on Electric Utility Deregulation, Restructuring and Power Technologies, Vol. 1, pp 393 397, April 2004

[50] H. Wan, K. K. Li and K. P. Wong, "An Multi-Agent Approach to Protection Relay Coordination with Distributed Generators in Industrial Power Distribution System," Conference Record of the Industry Applications 40 ${ }^{\text {th }}$ IAS Annual Meeting, Vol. 2, pp 830 - 836, October 2005

[51] L. Cristaldi, A. Monti, R. Ottoboni and F. Ponci, "Multi-agent based power systems monitoring platform: a prototype", in Proc. of IEEE Power Tech Conference, June, Vol. 2, 5pp., 2003

[52] B. Frances, C. Frank, G. Rune, M. Jonker, L. Olle , P. Bianca, T. Jan, "Agents Negotiating for Load Balancing of Electricity Use", Proceedings of the International Conference on Distributed Computing Systems, 1998

[53] A. Vale, R. Carlos Hugo Morais, F. Pedro, S. Marco "The Role of Demand Response in Future Power Systems", Transmission \& Distribution Conference \& Exposition: Asia and Pacific, 2009

[54] C.J. Warmer, M.P.F. Hommelberg, B. Roossien, J.K. Kok and J.W. Turkstra, "A field test using agents for coordination of residential micro-chp", International Conference on Intelligent Systems Applications to Power Systems, 2007

[55] T. Prakash, K. Jinho , B.Audun, N. Youngwoo, "Modeling and Simulation of Price Elasticity of Demand Using an Agent-Based Model", Innovative Smart Grid Technologies, 2010

[56] K. Morgan, H. Mullany, and M. Walsh, "Implementation of a Novel Peak Demand Reduction Scheme", Power Systems Conference and Exposition, 2004

[57] E. Crane, G. Lorsch, L. Oswald, "Thermal Storage of Off--Peak Electrical Energy in Solar Heating and Cooling Systems", IEEE Conference on Decision and Control including the 17th Symposium on Adaptive Processes, 1978 
[58] B. John, W. Young, N. Ralph, B. William, "Commercial Building Demand-Side Management Tools: Requirement for Dispatchable Photovoltaic Systems", Conference Record of the Twenty Third IEEE Photovoltaic Specialists Conference, 1993

[59] A. Aitouche and S. Hayat, "Multi-agent Model using Colored Petri Nets for the Regulation Traffic of an Automated Highway," IEEE Proceedings on Intelligent Transportation Systems, Vol. 1, pp 37 - 42, 2003

[60] K. Zafar, S. B. Qazi and A. R. Baig, "Mine Detection and Route Planning in Military Warfare using Multi Agent System," IEEE Proceedings of the 30th Annual International Computer Software and Applications Conference, Vol. 2, pp 327 - 332, September 2006

[61] H. Shaalan, J. Thompson, R. Broadwater, M. Ellis and H. Ng, "Distribution Engineering Tool Features a Flexible Framework," IEEE Transactions on Computer Applications in Power, Vol. 8, Issue 3, pp 21 - 24, July 1995

[62] R. Broadwater, J. Thompson, M. Ellis, H. Ng, N. Singh and D. Loyd, "Application Programmer Interface for the EPRI Distributed Engineering Workstation," Proceedings of IEEE Transactions on Power Systems, Vol. 10, No. 1, pp. 499 - 505, February 1995

[63] http://sourceforge.net/projects/electricdss/.

[64] http://www.smartgrid.epri.com/doc/OpenDSS Level 1 Training.pdf

[65] http://jade.tilab.com/

[66] B. Fleming, "Creating a Phase Current Unbalance Alarm/Supervision Function in the SEL-351 Relay," Schweitzer Engineering Laboratories, Inc., 1998

[67] F. Calero, "Rebirth of negative-Sequence Quantities in protective Relaying with microprocessor-Based relays," Schweitzer Engineering Laboratories, Inc., 2003

[68] A. F. Elneweihi, E. O. Scweitzer, and M. W. Feltis, "Negative-Sequence Overcurrent Element Application and Coordination in distribution Protection," IEEE Trans. On Power Delivery, Vol. 8, No. 3, pp. 915-924, July 1993

[69] Radial Distribution Test Feeders, http://www.ewh.ieee.org/soc/pes/dsacom/testfeeders.html 